\title{
INFLUENCE OF PROTOZOAN GRAZING \\ ON THE MARINE GEOCHEMISTRY \\ OF PARTICLE REACTIVE TRACE METALS
}

By

Katherine Barbeau

B.S., Southampton College, Long Island University

(1991)

SUBMITTED IN PARTIAL FULFILLMENT OF THE REQUIREMENTS

FOR THE DEGREE OF

0

0

0

DOCTOR OF PHILOSOPHY

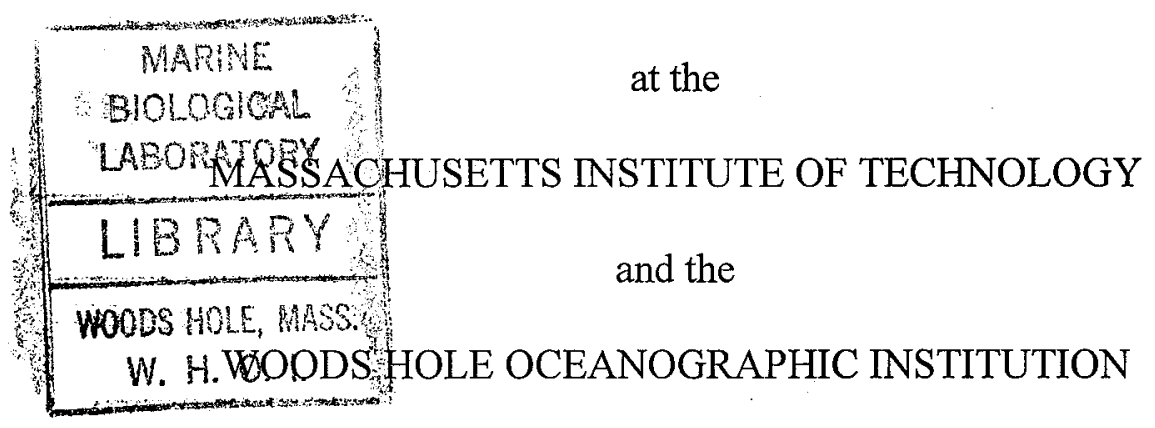

May 1998

(c) 1998 Katherine Barbeau.

All rights reserved.

The author hereby grants to MIT and WHOI permission to reproduce paper and electronic copies of this thesis in whole or in part, and to distribute them publicly.

Signature of Author

Joint Program in Oceanography, Massachusetts Institute of Technology/Woods Hole Oceanographic Institution

Certified by

James W. Moffett

Thesis Supervisor

Accepted by

Edward A. Boyle

Chairman, Joint Committee for Marine Chemistry and Geochemistry Massachusetts Institute of Technology/Woods Hole Oceanographic Institution 


\title{
INFLUENCE OF PROTOZOAN GRAZING ON THE MARINE GEOCHEMISTRY OF PARTICLE REACTIVE TRACE METALS
}

\begin{abstract}
Planktonic protozoan grazers have the potential to significantly affect the chemistry of particle-associated trace metals. This is due both to the importance of protists as consumers of bacterial-sized particles, and to the unique low-pH, enzyme-rich microenvironment of the grazer food vacuole. This thesis examines the role of protozoan grazers in the marine geochemistry of strongly hydrolyzed, particle-reactive trace metals, in particular Th and $\mathrm{Fe}$.
\end{abstract}

A series of tracer experiments was carried out in model systems in order to determine the effect of grazer-mediated transformations on the chemical speciation and partitioning of radioisotopes $\left({ }^{59} \mathrm{Fe},{ }^{234} \mathrm{Th},{ }^{51} \mathrm{Cr}\right)$ associated with prey cells. Results indicate that protozoan grazers are equally able to mobilize intracellular and extracellular trace metals. In some cases, protozoan regeneration of trace metals appears to lead to the formation of metal-organic complexes. Protozoan grazing may generate colloidal material that can scavenge trace metals and, via aggregation, lead to an increase in the metal/organic carbon ratio of aggregated particles.

Model system experiments were also conducted in order to determine the effect of grazers on mineral phases, specifically colloidal iron oxide (ferrihydrite). Several independent techniques were employed, including size fractionation of ${ }^{59} \mathrm{Fe}$-labeled colloids, competitive ligand exchange, and iron-limited diatoms as "probes" for bioavailable Fe. Experimental evidence strongly suggests that protozoan grazing can affect the surface chemistry and increase the dissolution rate of iron oxide phases through phagotrophic ingestion.

In further work on protozoan-mediated dissolution of colloidal Fe oxides, a novel tracer technique was developed based on the synthesis of colloidal ferrihydrite impregnated with ${ }^{133} \mathrm{Ba}$ as an inert tracer. This technique was shown to be a sensitive, quantitative indicator for the extent of ferrihydrite dissolution/alteration by a variety of mechanisms, including photochemical reduction and ligand-mediated dissolution. In field experiments using this technique, grazing by naturally occuring protistan assemblages was shown to significantly enhance the dissolution rate of colloidal ferrihydrite over that in non-grazing controls. Laboratory and field results indicate that, when integrated temporally over the entire euphotic zone, protozoan grazing may equal or exceed photoreduction as a pathway for the dissolution of iron oxides. 


\section{Acknowledgments}

Throughout the course of this thesis work, I have been fortunate to receive the invaluable assistance of numerous people. I would first like to thank my advisor, Jim Moffett, for his scientific insight, untiring patience, and unique sense of humor. I could not ask for a better mentor, colleague, or role model. I am indebted to the members of my committee, Dave Caron, Ed Boyle, Ken Buesseler, and Francois Morel for their interest in and commentary on my research. Dave in particular has always been available for discussion and a source of good advice. I thank Ed Sholkovitz for serving as chair of my defense, and for generally being supportive during my time at WHOI. John Farrington has also provided support and encouragement throughout my stay.

I owe Moffett lab members past and present, especially Liz Kujawinski, Mak Saito, Peter Croot, and Carla Bold for their friendship, practical assistance, and solidarity. I have received generous help from various people in the biology department, including Mark Dennett, Ee Lin Lim and Dawn Moran in the Caron lab; Deana Erdner and Dave Kulis in the Anderson lab; and John Waterbury and Freddie Valois. A number of individuals in MC\&G have also contributed to my research, including Alan Fleer and Mike Bacon, members of the Buesseler group, Ed Peltzer, and Ollie Zafiriou.

On a personal level, thanks are due to many in the Joint Program for their friendship, especially Lihini Aluwihare and Maria Hood; and to my housemates Liz Minor, Sue Bello, Lisa Max and Laura Magde. I am particularly grateful, more so than I can possibly express herein, to Tad Snow for his love and support in the past few years. Finally and most sincerely I would like to thank my family, especially my parents and my two sisters, for their love and for their encouragement of all my pursuits, however obsessive.

This work was financially supported by a Department of Defense ONR-NDSEG Graduate Fellowship, Office of Naval Research AASERT Award (N00014-94-1-0711), and the National Science Foundation EGB Program (OCE-9523910). 


\section{TABLE OF CONTENTS}

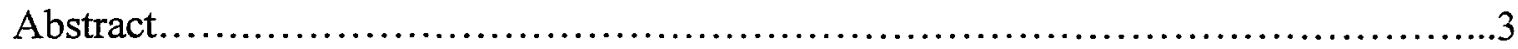

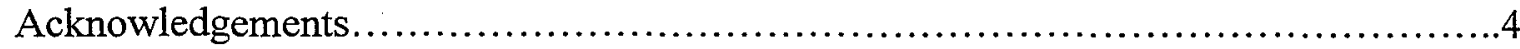

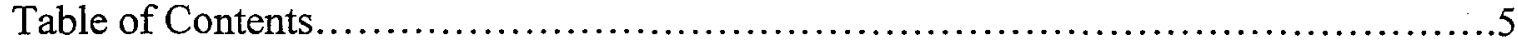

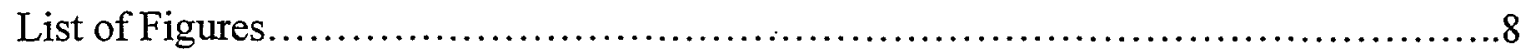

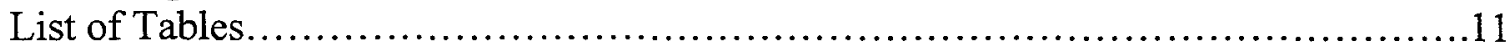

\section{Chapter 1: Introduction}

Overview......................................................... 13

Cycling of trace metals in the oceans - role of small particles and bacteria.14 The microbial food web and its role in planktonic nutrient cycles...........15 Potential importance of protozoan grazers in trace metal cycling...........19 Evidence for the role of protozoan grazers in trace metal cycling............23 Contextual synthesis and motivation for thesis research...................27

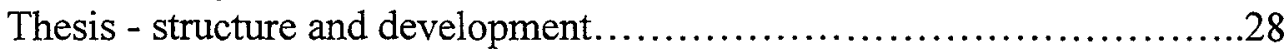

References..........................................................

Chapter 2: Remineralization and recycling of particle-reactive trace metals by protozoan grazers: Fate of cell-associated trace metals Introduction...........................................................

General Background.................................................

Selection of trace metals............................................39

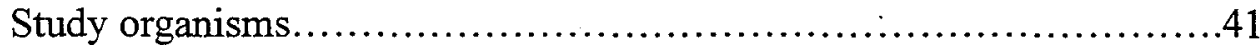

Basic experimental protocol........................................42

Overview of experiments..........................................43

Experimental.......................................................... 43

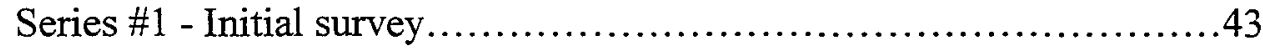

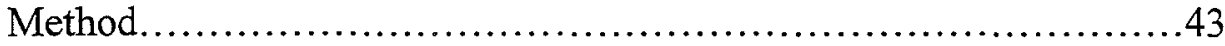

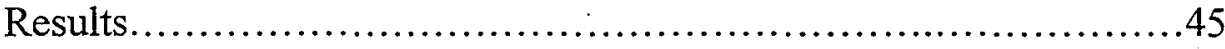

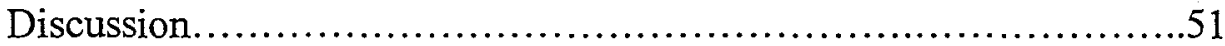

Series \#2 - Subsequent experiments...............................56

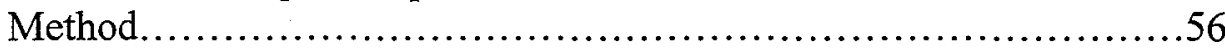

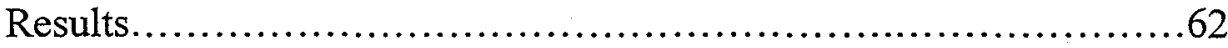

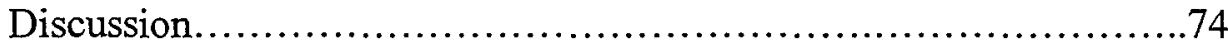

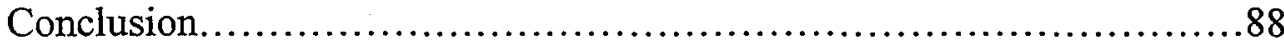

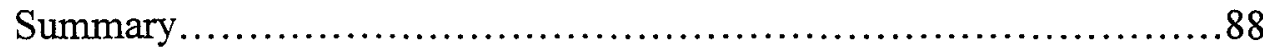

Application to natural systems...................................... 88

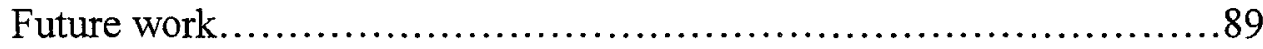

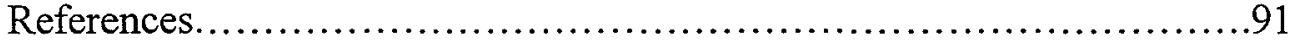

Chapter 3: Dissolution of colloidal iron oxides by protozoan grazers: Initial results Introduction...........................................................

General background............................................97

Processes influencing iron availability ...............................99

Experimental approach.......................................101 


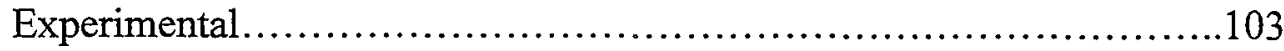

Colloid synthesis....................................................103

Size fractionation/oxine lability experiments.......................106

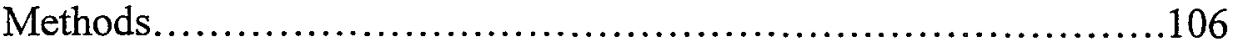

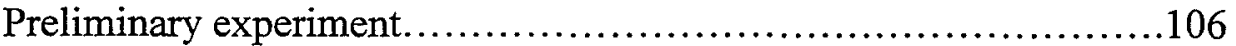

Second experiment............................................ 110

CLE/CSV with 1-nitroso-2-napthol...................................113

Methods............................................................

Preliminary experiment........................................114

Mechanistic experiments......................................116

Glucose-grown bacteria experiment..............................120

Heat-killed bacteria experiments................................124

Grazing culture filtrate experiment............................... 124

Experiments with iron-limited diatoms.............................130

Methods...........................................................

Initial experiments............................................ 137

Diatom experiments with heat-killed bacteria.......................141

Conclusion..........................................................

Summary ........................................................

Significance and caveats...........................................143

Protozoan grazers and "new" iron......................................145

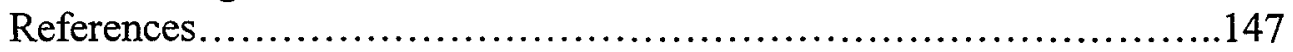

\section{Chapter 4: Development and application of a novel technique to study reactions of} synthetic iron oxides in seawater

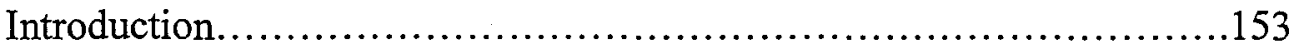

Iron oxides in surface waters.......................................153

Methods for studying reactions of iron oxides in seawater..............154

Novel method..........................................................156

Method development - Chapter overview..............................157

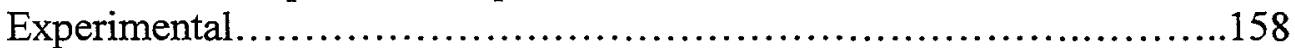

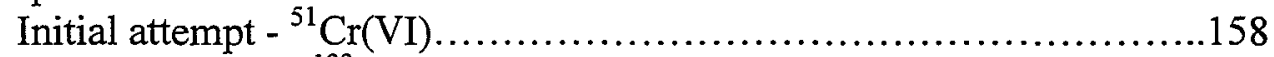

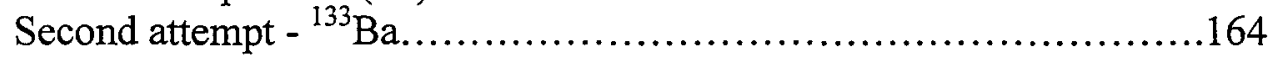

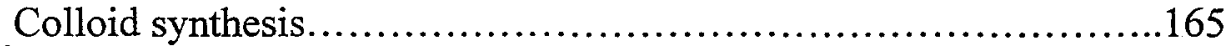

${ }^{133} \mathrm{Ba}$-impregnated ferrihydrite - dialyzed colloids.....................168

Dialysis protocol................................................ 168

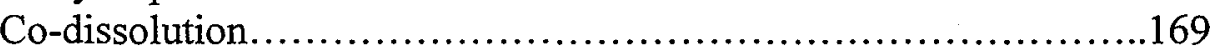

Dialyzed colloids - experimental applications.......................176

Photochemical experiments - irradiation system.....................176

Photochemical experiment - natural sunlight.......................187

${ }^{133} \mathrm{Ba}$-impregnated ferrihydrite - rinsed/resuspended colloids............192

Conclusion...............................................................195

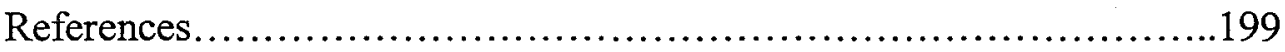


Chapter 5: Dissolution of colloidal iron oxides by protozoan grazers: Measurement of absolute dissolution rates in the laboratory and in the field

Introduction........................................................203

Previous work - the need for a new technique..........................203

Development of a new method.....................................205

Application of new method......................................206

Experimental.................................................207

Laboratory experiments with protists in culture......................207

Grazer-mediated Fe oxide dissolution with/without EDTA...........207

Rate and efficiency of grazer-mediated Fe oxide dissolution..........211

Species comparison experiment.................................215

Hcil experiment...............................................220

Interactive effects of grazing and light..........................221

Field experiments................................................225

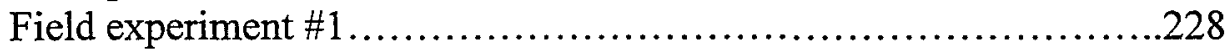

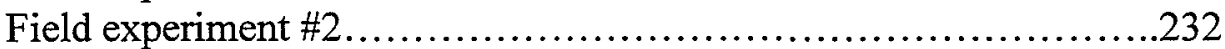

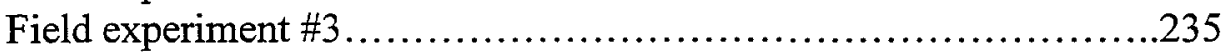

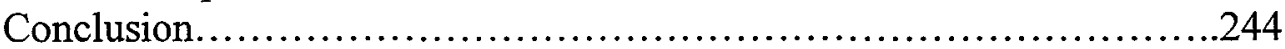

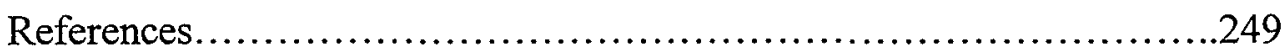

\section{Chapter 6: Synthesis and Conclusions}

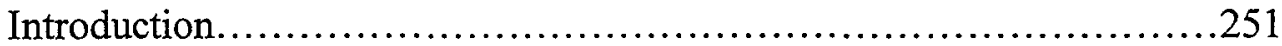

Specific conclusions/contributions of this thesis.....................252

Wider implications of this research..................................255

Phagotrophy as a vector for trace metal-biota interaction...............256

Suggestions for further research......................................259

References....................................................263 


\section{LIST OF FIGURES}

\section{Chapter 1}

Fig. 1.1: Schematic of colloidal pumping model..................................16

Fig. 1.2: Simplified schematic of marine microbial food web........................18

Fig. 1.3: Basic digestive process in a heterotrophic protist........................21

\section{Chapter 2}

Fig. 2.1: Series \#1, Uronema experiment, ${ }^{59} \mathrm{Fe}$ size fractions vs time.................46

Fig. 2.2: Series \#1, Uronema experiment, ${ }^{234} \mathrm{Th}$ size fractions vs time................47

Fig. 2.3: Series \#1, Paraphysomonas experiment, ${ }^{59} \mathrm{Fe}$ size fractions vs time...........48

Fig. 2.4: Series \#1, Paraphysomonas experiment, ${ }^{234} \mathrm{Th}$ size fractions vs time........49

Fig. 2.5: Series \#1, Paraphysomonas experiment, ${ }^{51} \mathrm{Cr}$ size fractions vs time.........50

Fig. 2.6: Series \#1, Cafeteria experiment, ${ }^{59} \mathrm{Fe}$ size fractions vs time.................52

Fig. 2.7: Series \#1, Cafeteria experiment, ${ }^{234} \mathrm{Th}$ size fractions vs time................53

Fig. 2.8: Series \#1, Cafeteria experiment, ${ }^{51} \mathrm{Cr}$ size fractions vs time................54

Fig. 2.9: Series \#2, Population dynamics. (A) Cafeteria experiment;

(B) Paraphysomonas experiment.

Fig. 2.10: Series \#2, Organic carbon size fractions vs time. Cafeteria experiment:

(A) control, (B) grazing culture. Paraphysomonas experiment: (C) control,

(D) grazing culture.

Fig. 2.11: Series $\# 2,{ }^{59} \mathrm{Fe}$ size fractions vs time. Cafeteria experiment:

(A) control, (B) grazing culture. Paraphysomonas experiment: (C) control,

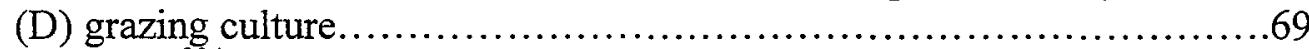

Fig. 2.12: Series $\# 2,{ }^{234}$ Th size fractions vs time. Cafeteria experiment:

(A) control, (B) grazing culture. Paraphysomonas experiment: (C) control,

(D) grazing culture ............................................... 70

Fig. 2.13: Series $\# 2,{ }^{51} \mathrm{Cr}$ size fractions vs time. Cafeteria experiment:

(A) control, (B) grazing culture. Paraphysomonas experiment: (C) control,

(D) grazing culture ................................................72

Fig. 2.14: Series \#2, Cafeteria experiment: (A) ${ }^{234} \mathrm{Th} / \mathrm{C}$, (B) ${ }^{59} \mathrm{Fe} / \mathrm{C}$ and $(\mathrm{C}){ }^{51} \mathrm{Cr} / \mathrm{C}$

ratios in $>0.2 \mu \mathrm{m}$ size class vs time.................................. 81

Fig. 2.15: Series \#2, Cafeteria experiment: (A) ${ }^{234} \mathrm{Th} / \mathrm{C}$ and (B) ${ }^{51} \mathrm{Cr} / \mathrm{C}$ ratios in $0.2-$

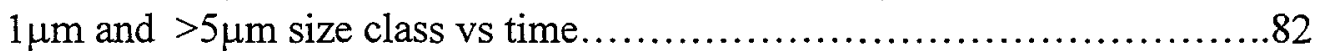

Fig. 2.16: Series \#2, Paraphysomonas experiment: (A) ${ }^{234} \mathrm{Th} / \mathrm{C}$, (B) ${ }^{59} \mathrm{Fe} / \mathrm{C}$ and

(C) ${ }^{51} \mathrm{Cr} / \mathrm{C}$ ratios in $>0.2 \mu \mathrm{m}$ size class vs time.

Fig. 2.17: Series \#2, Paraphysomonas experiment: (A) ${ }^{234} \mathrm{Th} / \mathrm{C}$ and (B) ${ }^{51} \mathrm{Cr} / \mathrm{C}$ ratios in

$0.2-1 \mu \mathrm{m}$ and $>5 \mu \mathrm{m}$ size class vs time.

\section{Chapter 3}

Fig. 3.1: SEM of concentrated colloidal sol at $70,000 \mathrm{x}$ and $100,000 \mathrm{x} \ldots \ldots \ldots \ldots \ldots . . .105$

Fig. 3.2: Data from preliminary experiment in size fractionation/oxine lability series.

(A) oxine lability at $\mathrm{t}_{31 \mathrm{~h}}$ and $\mathrm{t}_{61 \mathrm{~h}}$; (B) $\%$ dissolved ${ }^{59} \mathrm{Fe}$ vs time.............109

Fig. 3.3: Data from second experiment in size fractionation/oxine lability series.

(A) oxine lability vs time; (B) $\%$ dissolved ${ }^{59} \mathrm{Fe}$ vs time..................111 
Fig. 3.4: First experiment in CLE/CSV series, multi-species screen. 1N2N-labile Fe vs time in different grazing cultures....................................115

Fig. 3.5: Mechanistic experiments with CLE/CSV: 1N2N-labile Fe vs time.

(A) 50 FERR vs 90 FERR; (B) Bacteria concentration effect;

(C) EDTA concentration effect.

Fig. 3.6: CLE/CSV experiment with glucose-grown bacteria and Cafeteria as grazer:

$1 \mathrm{~N} 2 \mathrm{~N}-\mathrm{labile} \mathrm{Fe}$ (in $\mathrm{nM}$ and $\%$ total $\mathrm{Fe}$ ) vs time.......................122

Fig. 3.7: Extended incubation data from CLE/CSV experiment in Fig. 3.6.

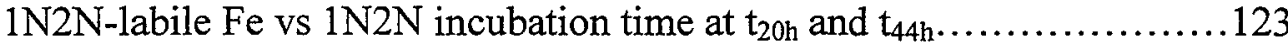

Fig. 3.8: Standard addition curves for CLE/CSV in model systems with heat-killed bacteria. Peak height (nA) vs [Fe] added.............................125

Fig. 3.9: CLE/CSV data from grazing culture filtrate experiment. 1N2N-labile Fe vs time in (A) monitoring cultures; (B) culture filtrates vs VSW .............128

Fig. 3.10: Second iron-limited diatom experiment with live bacteria as prey.

(A) $\mathrm{chl} a$ per diatom cell at $\mathrm{t}=4$ days; (B) diatom growth curves.

Fig. 3.11: Second iron-limited diatom experiment with heat-killed bacteria as prey.

(A) chl $a$ per diatom cell at $t=4$ days; (B) diatom growth curves.

\section{Chapter 4}

Fig. 4.1: Resin co-dissolution of ${ }^{51} \mathrm{Cr}$-impregnated ferrihydrite.

(A) $\%$ dissolved ${ }^{51} \mathrm{Cr}$ vs $\%$ dissolved ${ }^{59} \mathrm{Fe}$; (B) $\mathrm{cpm}^{59} \mathrm{Fe}$ and $\mathrm{cpm}{ }^{51} \mathrm{Cr}$

remaining in sol vs time.......................................... 160

Fig. 4.2: Photochemical experiments with ${ }^{51} \mathrm{Cr}$-impregnated ferrihydrite. $\%$ dissolved ${ }^{51} \mathrm{Cr}$ vs time in irradiated flasks and dark controls.............162

Fig. 4.3: Resin co-dissolution of ${ }^{133} \mathrm{Ba}$-impregnated ferrihydrite. $\%$ dissolved ${ }^{133} \mathrm{Ba}$ vs $\%$ dissolved ${ }^{59} \mathrm{Fe}$....

Fig. 4.4: Co-dissolution of ${ }^{133} \mathrm{Ba}$-impregnated ferrihydrite at $\mathrm{pH} 4$ in Sargasso seawater plus citrate. $\%$ dissolved ${ }^{133} \mathrm{Ba}$ vs $\%$ dissolved ${ }^{59} \mathrm{Fe}$.

Fig. 4.5: Co-dissolution of ${ }^{133} \mathrm{Ba}$-impregnated ferrihydrite in Sargasso seawater at $\mathrm{pH} 2.5 . \%$ dissolved ${ }^{133} \mathrm{Ba}$ vs $\%$ dissolved ${ }^{59} \mathrm{Fe}$

Fig. 4.6: Co-dissolution of ${ }^{133} \mathrm{Ba}$-impregnated ferrihydrite in Sargasso seawater via hydroxylamine reduction. $\%$ dissolved ${ }^{133} \mathrm{Ba}$ vs $\%$ dissolved ${ }^{59} \mathrm{Fe} . \ldots . .174$

Fig. 4.7: Absorbance spectra for (A) Sargasso seawater; (B) Vineyard Sound seawater; (C) Waquoit Bay water; (C) Salt Pond water............................ 179

Fig. 4.8: Photochemical kinetics experiments with ${ }^{133} \mathrm{Ba}$-ferrihydrite in SSW plus $100 \mu \mathrm{M}$ citrate at $\mathrm{pH} 6.5$ and $8 . \%{ }^{133} \mathrm{Ba}$ and ${ }^{59} \mathrm{Fe}$ dissolved vs time.

Fig. 4.9: Photochemical experiments with ${ }^{133} \mathrm{Ba}$-ferrihydrite incubated overnight in various waters at $4^{\circ} \mathrm{C}$ and $20^{\circ} \mathrm{C} . \%{ }^{133} \mathrm{Ba}$ dissolved in 1 hour irradiation...186

Fig. 4.10: Photochemical experiment with ${ }^{133} \mathrm{Ba}$-ferrihydrite in natural sunlight, whole and filtered VSW. Dissolved ${ }^{133} \mathrm{Ba}$ vs time.

Fig. 4.11: Bacterial dissolution experiment with rinsed ${ }^{133} \mathrm{Ba}$-ferrihydrite.

$\%$ dissolved ${ }^{133} \mathrm{Ba}$ vs time, with and without azide.

\section{Chapter 5}

Fig. 5.1: Grazer-mediated dissolution of ${ }^{133} \mathrm{Ba} /{ }^{59} \mathrm{Fe}$-ferrihydrite, with/without EDTA.

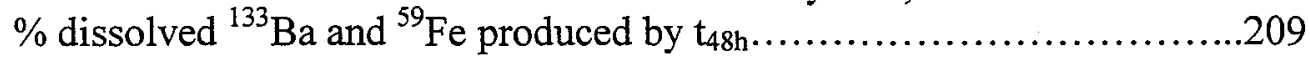


Fig. 5.2: Rate and efficiency of colloidal Fe oxide dissolution by Cafeteria.

(A) Population dynamics in grazing culture; (B) Production of dissolved

${ }^{133} \mathrm{Ba}$ in grazing culture relative to control.

Fig. 5.3: Dissolution of colloidal ferrihydrite by different protozoan species.

(A) $\%$ dissolved ${ }^{133} \mathrm{Ba}$ vs time, normalized to total at $\mathrm{t}_{0}$.

(B) $\%$ dissolved ${ }^{133} \mathrm{Ba}$ vs time, normalized to total at $\mathrm{t}_{\mathrm{x}}$

Fig. 5.4: Dissolution of colloidal ferrihydrite by Hcil. (A) Population dynamics;

(B) $\%$ dissolved ${ }^{133} \mathrm{Ba}$ vs time relative to control

Fig. 5.5: Interactive effects of grazing and light. $\%$ dissolved ${ }^{133} \mathrm{Ba}$ vs time in irradiated vs non-irradiated Cafeteria grazing cultures, relative to irradiated bacteria control.........................................................224

Fig. 5.6: Field experiment \#1. (A) Bacterial consumption in different treatments over time (B) $\%$ dissolved ${ }^{133} \mathrm{Ba}$ in different treatments at day $5 \ldots \ldots \ldots \ldots \ldots . .230$

Fig. 5.7: Field experiment \#3. Bacterial consumption in different treatments over

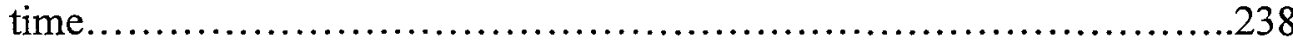

Fig. 5.8: Field experiment \#3. Loss of ${ }^{133} \mathrm{Ba}$ over time in different treatments, relative to ${ }^{133} \mathrm{Ba}$ total at $\mathrm{t}_{0}$. (A) Ferrihydrite added with bacteria; (B) Ferrihydrite

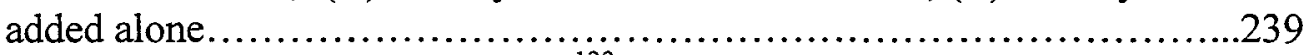

Fig. 5.9: Field experiment $\# 3 . \%$ dissolved ${ }^{133} \mathrm{Ba}$ vs time in different treatments, normalized to total ${ }^{133} \mathrm{Ba}$ at $\mathrm{t}_{0}$.

(A) Ferrihydrite added with bacteria; (B) Ferrihydrite added alone

Fig. 5.10: Field experiment \#3. \% dissolved ${ }^{133} \mathrm{Ba}$ vs time in different treatments, normalized to total ${ }^{133} \mathrm{Ba}$ at $\mathrm{t}_{\mathrm{t}}$.

(A) Ferrihydrite added with bacteria; (B) Ferrihydrite added alone 


\section{LIST OF TABLES}

\section{Chapter 2}

Table 2.1: Experimental series \#2, $\mathrm{pH}$ values for bacteria control and grazing cultures from $\mathrm{t}_{0}-\mathrm{t}_{48}$. Part A - Cafeteria as grazer; Part B - Paraphysomonas as

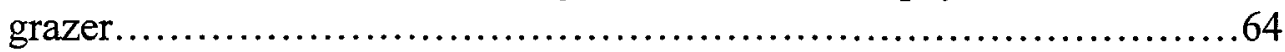

Table 2.2: Experimental series \#2, Ti(III) wash results for bacteria controls in Parts A

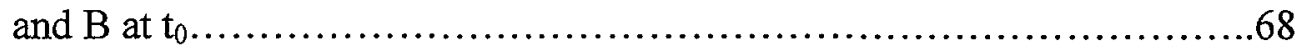

Table 2.3: Experimental series \#2 - vacuum filtration, reverse-flow filtration, and

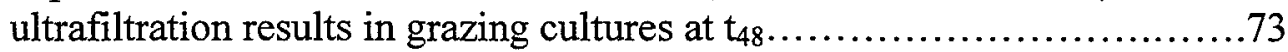

\section{Chapter 3}

Table 3.1: Initial experiment with iron-limited diatoms. Diatom cell concentrations and per cell chl $a$ concentrations at day 4 of experiment...................134

Table 3.2: Bulk chl $a$ measurements from first iron-limited diatom experiment using heat-killed bacteria as prey for protists. Values shown for day 5 of experiment

\section{Chapter 4}

Table 4.1: Photochemistry in natural waters. $\%$ dissolved ${ }^{133} \mathrm{Ba}$ produced in 1 -hour $\mathrm{Hg} / \mathrm{Xe}$ lamp irradiations of ${ }^{133} \mathrm{Ba}$-ferrihydrite in various water types.....181

Table 4.2: Thermal effects. \% dissolved ${ }^{133} \mathrm{Ba}$ in samples incubated 24 hours at $4^{\circ} \mathrm{C}$

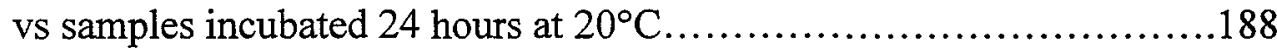

\section{Chapter 5}

Table 5.1: Data from species comparison grazing experiment. Protozoan cell yields, $\%$ bacteria consumed, excess $\%{ }^{133} \mathrm{Ba}$ dissolved, and consumptionnormalized efficiency for each species..................................219

Table 5.2: Size fractions and treatments set up for field experiment incubations......227

Table 5.3: Size fractions and treatments set up for field experiment \#3 incubations..236 


\section{CHAPTER 1}

\section{Introduction}

\section{Overview}

The study of how trace metals and biota interactively influence each other in the marine environment is continually developing as advances are made both in our understanding of trace metal chemistry in seawater and in our conception of marine ecological processes. Our knowledge of the biogeochemical cycling of trace metals in the oceans has increased significantly in the past 20 years, due to a combination of increased sensitivity in analytical techniques and improvements in laboratory culture methods (Bruland et. al., 1991). During the same 20 year period, our understanding of trophic relationships and nutrient cycling in the planktonic ecosystem has changed tremendously due to the realization of the significance of the microbial food web in the marine water column (Valiela, 1995). At the convergence of these two advancing paradigms, trace metal chemists and microbial ecologists are working together to define how trace metals interact with the microbial ecosystem in the upper oceans, and how these interactions affect the planktonic ecosystem as a whole.

The work described in this thesis is part of the larger effort to characterize the interaction of trace metals with the microbial food web. This research focuses on how the grazing activities of protists (microzooplankton) in the nanoplanktonic size class (2$20 \mu \mathrm{m}$ ) can influence the marine geochemistry of particle reactive trace metals. This introductory chapter will set the stage for those to follow by describing how this study fits into the current context of trace metal geochemistry and marine microbial ecology. Chapter 2 describes an investigation of how grazing by nanoplanktonic protists can influence the fate of particle reactive trace metals associated with bacterial prey cells. In Chapter 3, initial studies of the dissolution of colloidal iron oxides as mediated by protozoan grazing are described. Chapter 4 details the development and preliminary application of a novel method to study the dissolution of iron oxides in seawater. This method is applied to laboratory and field measurements of the rate of iron oxide 
dissolution by protists in Chapter 5 . Chapter 6 summarizes the results and contributions of the thesis as a whole, and includes some suggestions for further research.

\section{Cycling of trace metals in the oceans - role of small particles and bacteria}

With the advent of trace metal clean sampling methods, chemical oceanographers were able to accurately measure the distribution of trace metals in the oceans (reviewed by Bruland, 1983). In conjunction with field studies of trace metal distribution, culture studies using synthetic chelators demonstrated that the bioavailability of trace metals is related to the free metal ion concentration (reviewed by Sunda 1988-1989). The subsequent development of highly sensitive and specific trace metal speciation methods has confirmed the presence of dissolved organic chelators in ocean waters that form strong complexes with a variety of trace metals (eg. Bruland et. al., 1991). The existence of dissolved ligands and their mediation of trace metal bioavailability is now a major theme in our modern concept of trace metal/biota interactions in the marine environment. Ligands are also known to exist on particles, either as high affinity receptors located on cell surfaces for the purpose of trace metal acquisition (Hudson and Morel, 1990), or as less specific functional groups which can bind to trace metals (Balistrieri et. al., 1981).

If particles can be defined as a ligand pool, then the competition for trace metals between particulate and dissolved phase ligands determines the scavenging residence time of trace metals in the oceans. These ideas, developed in the late 70's and early 80's, (Schindler, 1975; Balistrieri et. al., 1981) are central to the application of surface complexation theory to the oceanic cycling of trace metals (Honeyman, 1991). Initial scavenging models based on the observation of ${ }^{234} \mathrm{Th}$ in surface waters and ${ }^{230} \mathrm{Th}$ in deeper waters proposed a dynamic equilibrium between Th in the particulate and dissolved phase (Bacon and Anderson 1982; Nozaki et. al. 1987). Until fairly recently, the focus in such scavenging models was on particles larger than a few microns in size. Observations confirmed that the primary removal of Th from surface waters is via sinking particles (Coale and Bruland 1985; Murray et. al 1989), and number of studies have focused on the role of fast-sinking copepod fecal pellets in the scavenging removal of trace metals (Small and Fowler 1973; Fisher et. al., 1991). 
More recent theories have emphasized the role of very small particles and colloidal material in adsorbing trace metals from solution and then serving as a vector for the transfer to larger size classes via aggregation (Honeyman and Santschi 1989; Baskaran and Santschi 1993). Figure 1.1 shows a schematic of the "colloidal pumping" model proposed by Honeyman and Santschi, 1989. These processes are now thought to be the rate-limiting step in the scavenging removal of particle reactive trace elements from the oceanic water column. Studies of ${ }^{234} \mathrm{Th}$ in surface waters indicate that the small particles and colloids responsible for scavenging this element must be removed from surface waters relatively quickly, on time scales of weeks to a month (Moran and Buesseler, 1992). This implies an active cycling, which may be related to biological processes.

At the small end of the size spectrum, the surface area of biological particles is dominated by bacterial biomass. These organisms have a high surface area to volume ratio and an active surface chemistry, being covered with bound polysaccharides and proteoglycans (Lee et. al., 1993). Bacteria have been proposed as major scavengers of

${ }^{234} \mathrm{Th}$, and also as important scavengers of colloidal material (Cho and Azam, 1988; Lee et. al, 1993). In addition to their importance in the adsorption of particle reactive trace metals like Th, bacteria also likely play a major role in the active uptake of bioactive trace metals. Heterotrophic bacteria, for example, have recently been shown to have a high metabolic requirement for Fe (Tortell et. al., 1996). It is likely that bacteria compete directly with phytoplankton for the active uptake of $\mathrm{Fe}$ and other trace metal nutrients using transport sites on their external surfaces (Neilands, 1981). Thus, bacteria play a major role in both the active and passive scavenging of trace metals in the marine environment. Once associated with bacterial cells, the fate of these trace metals is to be biologically cycled through the microbial food web.

\section{The microbial food web and its role in planktonic nutrient cycles}

The emergence of the microbial loop as a central paradigm of the oceanic food web has resulted in vast changes both in our concept of the trophic relationships within the planktonic ecosystem, and in our understanding of nutrient cycling within this ecosystem. 


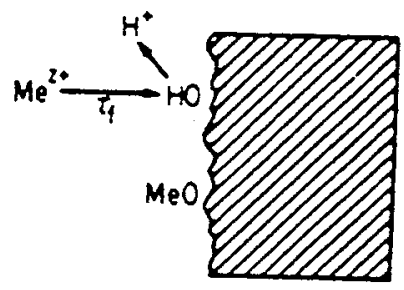

(a)

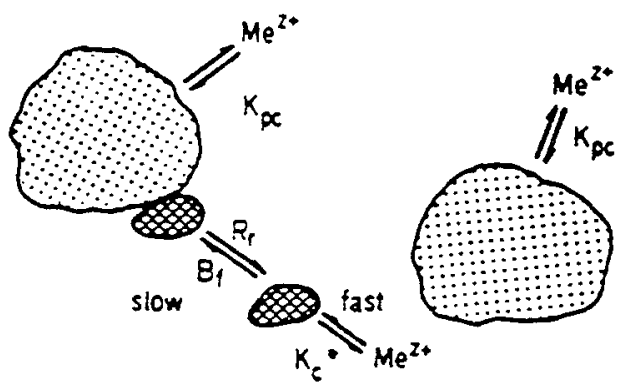

(b)

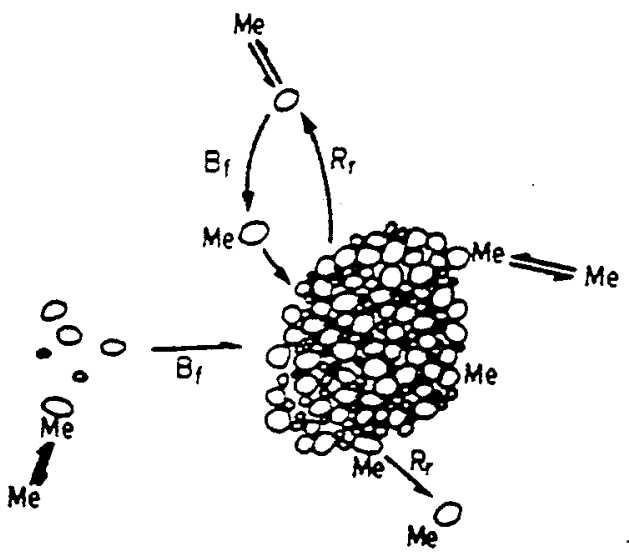

(c)

Figure 1.1 - Adapted from Honeyman, 1991.

Schematic of "colloidal pumping model" of coagulation coupled to adsorption (Honeyman and Santschi, 1989).

a) Rapid sorption of trace elements onto particle surfaces.

b) Sorption by formation of surface complexes $\left(\mathrm{K}_{\mathrm{pc}}\right.$ and $\mathrm{K}_{\mathrm{c}}{ }^{*}$ representing conditional sorption constants) is fast relative to aggregation of colloids $\left(\mathrm{B}_{\mathrm{f}}\right)$.

c) Natural particle clusters contain materials of varied size, history and composition. 
This revolution began in 1974 with Pomeroy's proposal of a new model for the marine food web based on the concept of a microbial food loop. The subsequent development of improved microscopic techniques to estimate bacterial biomass and productivity supported this model by demonstrating that up to $50 \%$ of the carbon fixed by photosynthesis may be consumed by bacteria, rather than macrozooplankton (Azam et. al., 1983). Control of bacterial biomass by the grazing activities of small heterotrophs has been confirmed, (Goldman, 1984; Sieburth, 1984), and more recent research suggests that viral infection may also constitute an important bacterial sink (Proctor and Fuhrman, 1990). Thus the classical view of an ecosystem circumscribed by the uptake of inorganic nutrients by phytoplankton with subsequent consumption by metazoan grazers and transfer to higher trophic levels was replaced by one in which bacteria and their consumers played a significant role.

Further research has increasingly modified our concept of the microbial loop. This process has been facilitated by the improvement in techniques for sampling microheterotrophs and characterizing their grazing and production rates. A simplified schematic of the microbial food web is shown in Figure 1.2. The composition of the microbial community is organized on the basis of size classes into pico- $(0.2-2 \mu \mathrm{m})$, nano$(2-20 \mu \mathrm{m})$, and microplankton $(20-200 \mu \mathrm{m})$. While greatly simplified, this concept of size classes provides a convenient structural basis for understanding a complex food web. This food web, published by Fenchel in 1987, basically epitomizes the current concept, although to be more complete small ciliates should be included among the nanoplanktonic consumers (Sherr et. al., 1986), and viral mortality could be added as an additional bacterial sink (Proctor and Fuhrman, 1990). In addition, regeneration of nutrients by grazing organisms is not indicated.

Most of the more recent research on the microbial loop has centered on its function in the cycling of nutrients, particularly with regards to the role of the heterotrophic protists. Protozoan grazers became a focus of research on nutrient remineralization largely as a result of findings that bacteria do not excrete significant amounts of remineralized inorganic nutrients during active growth (eg. Hagstrom and Larsson, 1984). In addition, 


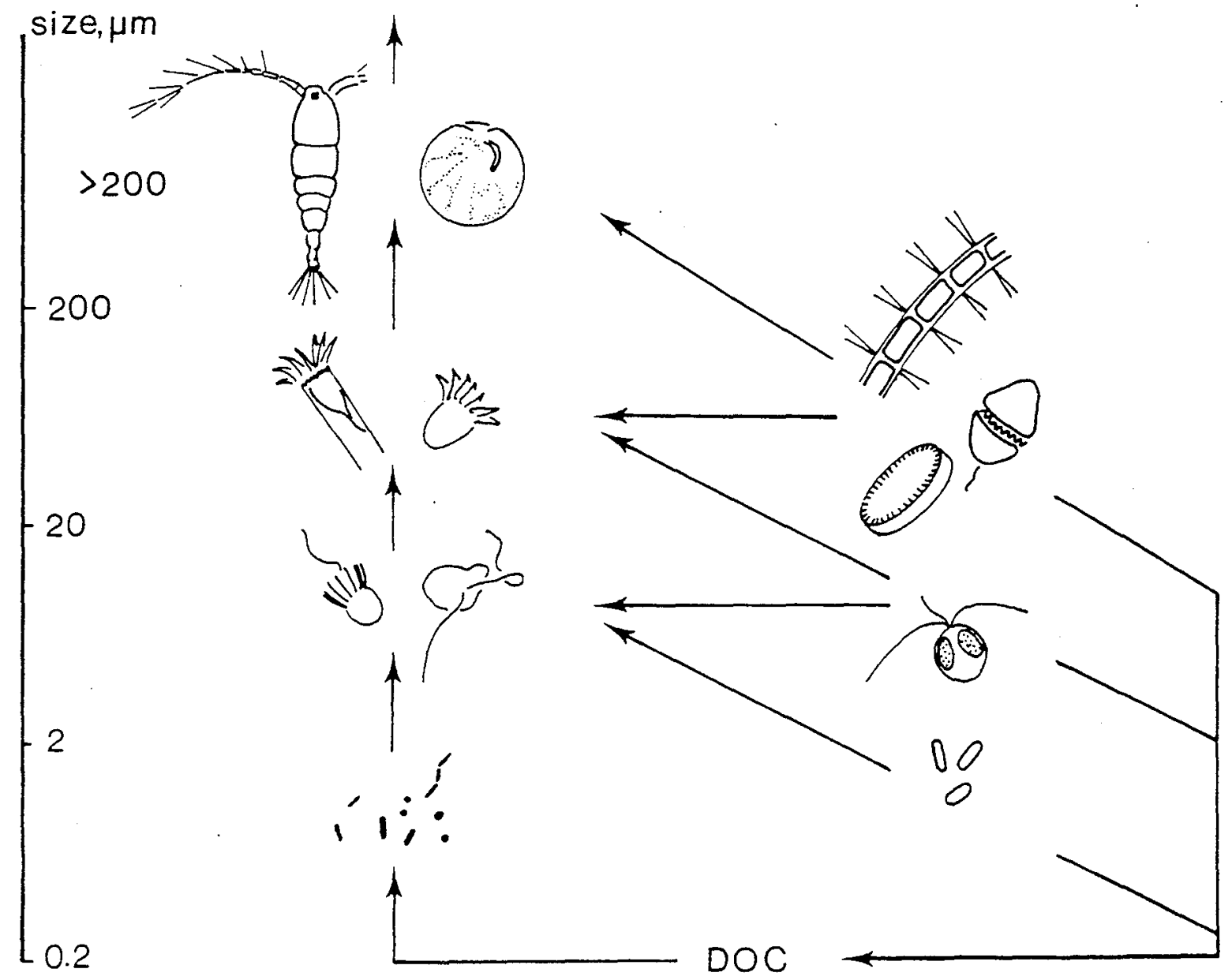

Figure 1.2 - Adapted from Fenchel, 1987.

Simplified schematic of planktonic marine microbial food web.

DOC $=$ dissolved organic carbon 
the importance of macrozooplankton grazers as a source of regenerated nutrients was called into question (Jackson, 1980).

The significance of protozoan grazers in the regeneration of nutrients $(N, P$, and organic carbon) was elegantly demonstrated in a series of laboratory model systems employing a heterotrophic bacterium, a small diatom, and a heterotrophic microflagellate (Goldman et. al., 1985; Caron et. al., 1985; Andersen et. al., 1986). Subsequent research (Caron et. al., 1988) demonstrated that the relative importance of bacteria and protozoa as a source of regenerated nutrients is heavily dependent on the carbon:nutrient ratio of the bacterial substrate, with protozoa being the more important nutrient regenerators when the carbon:nutrient ratio is high. The balance between protozoa and bacteria as nutrient remineralizers is thus somewhat complex and most likely variable. An easier evaluation of the remineralization potential of small protozoa vs. larger grazers is possible based on consideration of weight-specific metabolic rate and organism size, with the result that small, nano- and picoplanktonic protists should be more efficient remineralizers than larger protists and metazoan grazers (Caron, 1990). There is as yet no information available on the significance of viral mortality of bacteria as a source of remineralized nutrients.

\section{Potential importance of protozoan grazers in trace metal cycling}

As mentioned above, research on the ecology of the microbial food web has shown that heterotrophic protists in the nanoplanktonic size class are the primary consumers of bacteria, cyanobacteria, and small algae in the marine environment. They have also been implicated as major nutrient remineralizers in the planktonic ecosystem. These protists concentrate food particles from their immediate surroundings by a variety of strategies, discriminating primarily on the basis of size and retaining particles in the size range roughly $0.1-1 \mu \mathrm{m}$ (Fenchel, 1987). This includes both unicellular organisms and detrital particles, as well as smaller particles and colloids associated with the surface of larger particles or flocs colonized by protists. Based on current models of surface chemistry and trace element scavenging as described previously (see pp. 2-3), particles in this $0.1-1 \mu \mathrm{m}$ 
size class constitute a major pool of active surface area in marine systems. This surface area represents a major reservoir of particle reactive trace metals for which ingestion by small protozoan grazers is a primary sink.

Heterotrophic protists ingest particles by means of phagocytosis, a process in which the plasma membrane is expanded to engulf particles as large as a few micrometers (and sometimes larger). Once incorporated into a phagotrophic vacuole, intracellular digestion of particles (and their associated trace metals) begins. Despite the immense variety of unicellular phagotrophic organisms known to occur in nature, very few have been thoroughly characterized with respect to the mechanistics of intracellular digestion. The organism whose digestion is known in greatest detail is the ciliate Paramecium. This is largely the result of a series of studies conducted by Richard Allen and Agnes Fok over the past 20 years (eg. Fok et. al, 1982,1984,1985; Allen and Fok 1984; Ishida et. al. 1997). These workers have described the digestive process in Paramecium in great detail, defining various vacuole stages and determining $\mathrm{pH}$ and level of enzymatic activity in each. A schematic of this digestive cycle is shown in Figure 1.3.

The entire digestive cycle in Paramecium ranges from 20 to 60 minutes for individual vacuoles. In the first phase of digestion ( $\leq 5 \mathrm{~min}$ ), the newly-formed food vacuole condenses, and $\mathrm{pH}$ within drops from 7 to $\sim 3$. The vacuole remains in this condition for 3-4 minutes, then $\mathrm{pH}$ begins to rise and the vacuole expands as it fuses with lysosomes bearing digestive enzymes. During this period, when the vacuoles are between 8 and 20 minutes old, $\mathrm{pH}$ rises from 3 to 7 , and acid phosphatase activity is observed. Protistan acid phosphatase activity has a pH optimum of about 5 (Nagata and Kirchman, 1992). During this period of vacuole expansion and neutralization, digestive enzymes are active. Vacuoles older than about 20 minutes are at neutral $\mathrm{pH}$ with no digestive activity, and are presumably ready for defecation.

Although Paramecium makes a good model organism for studies of intracellular digestion, extreme departures from this model are likely to be found in nature. Digestion of the flagellate Chlorogonium elongatum by the ciliate Climacostomum virens, for example, has been shown to last 12-24 hours (Fischer-Defoy and Hausmann, 1982). The 


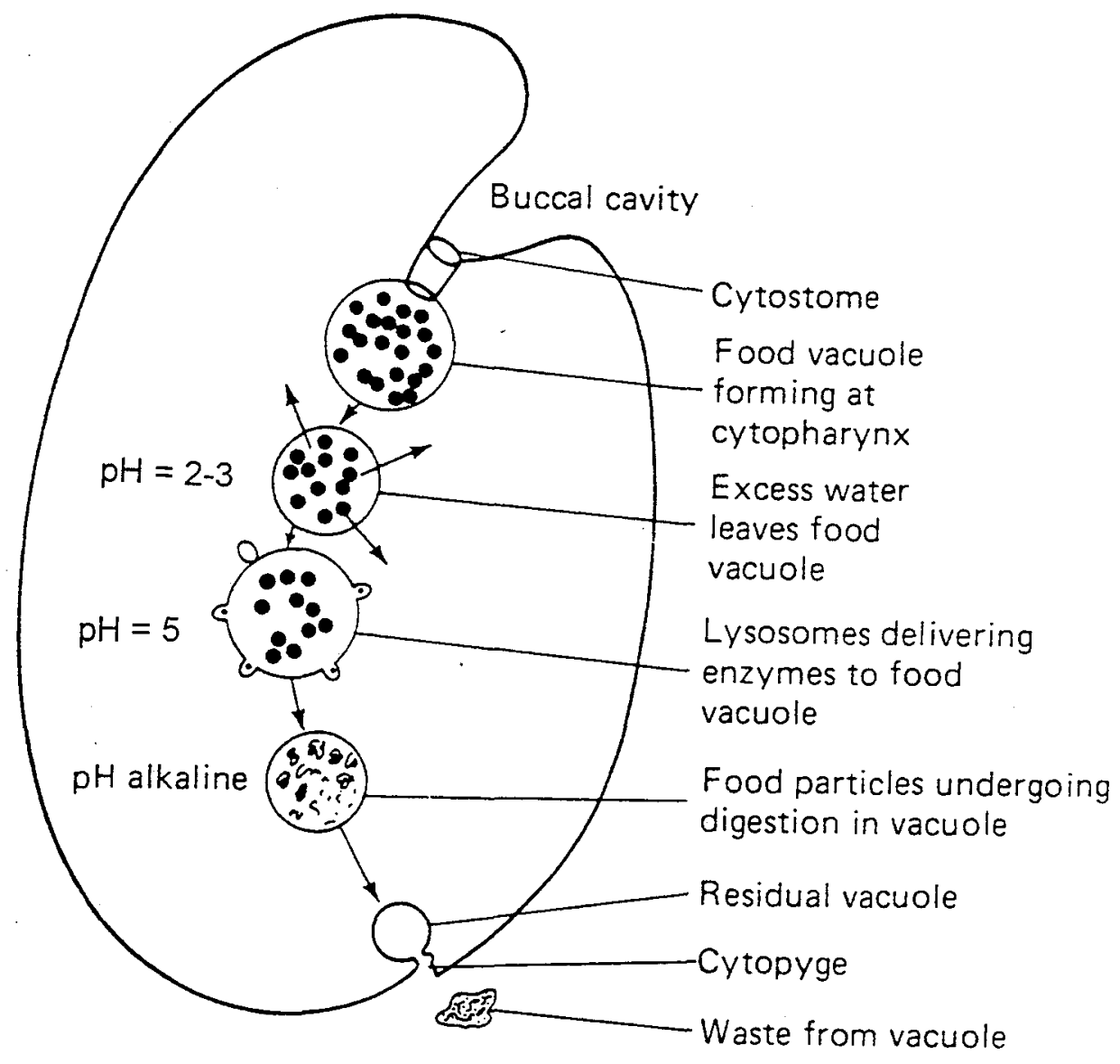

Figure 1.3 - Adapted from Adey and Loveland, 1991.

Basic digestive process in a heterotrophic protist. 
ciliate Pseudomicrothorax dubius, in contrast, appears to be able to dissolve filamentous algae almost instantaneously during the ingestion process. This organism has been hypothesized to possess rapid-acting, peroxide-based "killing agents" in addition to slower digestive enzymes (Peck and Hausmann, 1980).

Whatever variations may exist, all phagotrophic (intracellular) digestive processes have in common the characteristics of lowered $\mathrm{pH}$ (pH 5 is optimal for lysosomal enzymes) and intense enzymatic activity for some period of time (Mellman et. al., 1986). Phagocytosis has also been shown to involve elevated levels of reactive oxygen species like hydrogen peroxide and superoxide (Stossel, 1974). All of these conditions occur, of course, in the presence of high concentrations of reactive organic material. Particleassociated trace metal species entering into such an environment could certainly experience changes in redox state and/or coordination chemistry. These changes may have important effects on the mobility and bioavailability of trace metal species. Frey and Small (1979), for example, found that physical disruption of cells plus acid treatment caused a significant release of bioavailable iron from the cells. Cells that were just disrupted without acid treament released much less bioavailable iron.

The potential of small protozoan grazers to influence the geochemical cycling of trace metals in the marine environment is readily apparent. Because of their small size and method of feeding, protists access a large pool of trace metals associated with particles in the $0.1-1 \mu \mathrm{m}$ size class. Due to their digestive physiology, protists subject trace metals ingested with food particles to an intense chemical cycle that can cause changes in metal redox state and speciation. By comparison, larger grazers access a much smaller pool of particle reactive trace metals because they typically consume much larger particles (i.e. less surface area). In addition, the extracellular digestive cycle of larger grazers may be chemically less intensive than the intracellular digestion of protists. Food materials have a relatively short gut residence time in copepods (<30min; Reinfelder and Fisher, 1991) during which $\mathrm{pH}$ remains circumneutral (Pond et. al., 1995); although enzymatic activity is intense (Vonk, 1960). Studies have shown that copepods basically feed by 
mechanically crushing prey cell walls and absorbing soluble cytoplasmic components (Reinfelder and Fisher, 1991).

Aside from direct chemical transformations associated with ingestion and digestion, particle reactive trace metals may also be strongly influenced by protozoan grazing due to its overall effect on the natural particle assemblage. Stramski et. al. (1992) demonstrated that grazing dramatically alters the particle size distribution in model systems, converting bacterial-sized particles into larger and smaller particles. Moreover, protozoans may be an important source of colloidal material in the marine environment. Several studies (Nagata and Kirchman, 1992; Tranvik, 1994) have shown that protozoan grazers produce colloidal organic material. This comes from the ejecta of protozoan food vacuoles (i.e. waste), and appears to be in the form of liposome-like particles, high concentrations of enzymatic proteins surrounded by fragments of bacterial membranes (Nagata and Kirchman, 1992). The incorporation of particle reactive trace metals into colloidal particles by protozoan grazing could have important consequences for trace metal fate and transport (eg. Honeyman and Santschi, 1989).

\section{Evidence for the role of protozoan grazers in trace metal cycling}

Although large zooplankton grazers like copepods have been a subject of interest with regard to trace element cycling for a number of years (eg. Small and Fowler, 1973; Fowler, 1977), it is only relatively recently that microzooplankton (i.e. protozoan grazers) have been considered in this context. This is most likely a consequence of the relatively recent recognition of the importance of the microbial food web in upper ocean nutrient cycles.

In 1987 Coale and Bruland characterized ${ }^{234} \mathrm{Th} /{ }^{238} \mathrm{U}$ disequilibrium in the upper water column at VERTEX stations 2,3, and 4. Based on interpretation of the observed disequilibrium profiles, they postulated a two-layer euphotic zone: An upper oligotrophic layer with low levels of new production, intense recycling, and low ${ }^{234} \mathrm{Th}$ scavenging rates; and a subsurface eutrophic layer with higher levels of new production and more intense ${ }^{234} \mathrm{Th}$ scavenging. The authors invoked recycling due to "metazoan and microbial 
particle destruction" as an explanation for the enhanced residence time of ${ }^{234} \mathrm{Th}$ in the upper stratified euphotic zone. This oligotrophic, rapidly recycling surface regime was probably dominated by a microbial food web, in which the grazers most likely to account for the particle $/{ }^{234} \mathrm{Th}$ recycling were protozoans, rather than metazoans. A similar explanation could perhaps be invoked to (at least partially) account for the increased accumulation of $\mathrm{Pb}$ and ${ }^{210} \mathrm{~Pb}$ in the mixed layer observed in the oligotrophic Sargasso Sea during summertime stratification (Boyle et. al., 1986). These waters are also dominated by a microbial consortium during stratified conditions.

Some of the first direct evidence for the importance of microzooplankton grazers in mediating trace metal recycling came from the work of Hutchins et. al., (1993). Using radiolabeled cyanobacteria and diatoms added to fresh samples from open ocean and coastal waters, Hutchins et. al. demonstrated transfer of labeled Fe from cyanobacteria to larger cells $(>5 \mu \mathrm{m})$ in both environments. Although the mechanism of this transfer was not directly determined, the authors hypothesized that grazing of cyanobacteria by heterotrophic protists with subsequent $\mathrm{Fe}$ regeneration could account for the observed results. In a follow-up culture study employing ${ }^{55} \mathrm{Fe}$-labeled Synechococcus sp. and the microflagellate grazer Paraphysomonas sp., Hutchins and Bruland (1994) were able to demonstrate some remineralization from the cyanobacteria to the dissolved $(<0.2 \mu \mathrm{m})$ phase, apparently due to grazing activity. This evidence was taken as tentative, however, due to the possibility of lysis of fragile protozoan cells during filtration. In further field experiments using Synechococcus sp. labeled with ${ }^{59} \mathrm{Fe},{ }^{65} \mathrm{Zn},{ }^{54} \mathrm{Mn}$, and ${ }^{15} \mathrm{~N}$, Hutchins and Bruland compared the relative transfer efficiency of these various tracers from cyanobacteria to larger size classes $(>5 \mu \mathrm{m}$ and $>8 \mu \mathrm{m})$. They found that Fe was transferred into intracellular pools more efficiently than $\mathrm{Zn}$ and $\mathrm{Mn}$, and $70 \%$ as efficiently as $\mathrm{N}$ (Hutchins and Bruland, 1995). Results appeared to be related to the size of the ambient metal pool and phytoplankton metal demand relative to the amount of metal added as tracer.

Similar work in a freshwater system was done by Twiss and Campbell, 1995. In laboratory studies of a chrysophycean nanoflagellate (Ochromonas danica) grazing on a 
cyanobacterium (Synechococcus leopoliensis) labeled with ${ }^{65} \mathrm{Zn},{ }^{109} \mathrm{Cd},{ }^{153} \mathrm{Gd}$ and ${ }^{137} \mathrm{Cs}$, rapid regeneration of $\mathrm{Cd}$ and $\mathrm{Zn}$ into the dissolved $(<0.2 \mu \mathrm{m})$ phase was observed as a result of grazing. These experiments included controls to ensure that the apparent remineralization was not due to filtration artifacts. Ultrafiltration results demonstrated that $\mathrm{Zn}$ partitioned into the colloidal size class (as defined by 5000MWCO) more extensively than $\mathrm{Cd}$. Regenerated trace metals appeared to be less available for resorption by plankton than inorganic forms of the radionuclides. In field work on Lake Erie (Twiss et. al. 1996) regeneration of ${ }^{65} \mathrm{Zn}$ and ${ }^{109} \mathrm{Cd}$ from radiolabeled cyanobacterial cells into the dissolved $(<0.2 \mu \mathrm{m})$ phase was observed, apparently due to the grazing activities of the natural protistan consortium. The amount of trace metals regenerated into the dissolved phase was increased by the addition of $2 \mu \mathrm{M}$ EDTA.

Regeneration of trace metal micronutrients by protistan microzooplankton is now a more widely accepted concept. This is due partly to the studies described above by Hutchins and Twiss, and also due to general recognition of the role of heterotrophic protists in nutrient cycling. Recent studies of phytoplankton regulation in high-nutrient, low-chlorophyll (HNLC) regions of the oceans have in particular served to emphasize the role of protozoan grazing in the cycling of Fe. Landry et. al. (1997) published a synthesis of results obtained from the U.S. JGOFS EqPac Program and related studies of the HNLC central equatorial Pacific. In this regime where primary production is dominated by small phytoplankton, Landry et. al. concluded that while the rate of new iron input determines the amount of new production in the system, efficient recycling of iron by microzooplankton grazers determines the the total production of the system. These results were suggested to be similar in concept to the generally accepted view of the oligotrophic subtropical gyre ecosystem: A system dominated by small phytoplankton that are efficiently cropped by microzooplankton, where phytoplankton growth rates are tightly coupled to the remineralization of nutrients by grazers.

Despite the apparent analogy between iron cycling in the HNLC equatorial Pacific and major nutrient cycling in the subtropical gyres, questions remain about the degree of similarity between the two, and the role of heterotrophic protists in each. Related to these 
questions, Chase and Price (1998) found in a recent study of iron deficiency in heterotrophic marine protozoa that while the protists were apparently susceptible to $\mathrm{Fe}$ limitation, their metabolic response to that limitation was somewhat unexpected. When grazing on Fe-limited bacterial prey, the assimilation efficiency of the protists for $\mathrm{Fe}$ went down by about a factor of two, and regeneration of Fe to the dissolved $(<0.2 \mu \mathrm{m})$ phase went up significantly. These results contrast sharply with studies of protozoan $\mathrm{N}$ and $\mathrm{P}$ regeneration when consuming $\mathrm{N}$ - and $\mathrm{P}$ - limited prey (Goldman et. al. 1985, 1987), where protists were found to regenerate $\mathrm{N}$ and $\mathrm{P}$ less efficiently when grazing on $\mathrm{N}$ - and P- limited prey. This suggests that there may be fundamental differences in the recycling of $\mathrm{N}$ and $\mathrm{P}$ vs Fe by protozoan grazers, particularly under nutrient-limiting conditions. These differences could be related to differences in the bioavailability of regenerated forms of $\mathrm{Fe}, \mathrm{N}$ and $\mathrm{P}$ under conditions of nutrient limitation.

In addition to differences in their regenerated species, the major nutrients also differ substantially from $\mathrm{Fe}$ with respect to the form in which they are supplied to the euphotic zone from exogenous sources. This is particularly true for the HNLC equatorial Pacific, where recent research has shown that Fe is supplied to the euphotic zone primarily via the upwelling of Fe-rich water from the Equatorial Pacific Undercurrent (Coale et. al. 1996; Gordon et. al. 1997). This Fe has been hypothesized to originate from hydrothermal venting in the western Pacific, near the source waters of the Equatorial Undercurrent. If it is of lithogenic origin, the upwelled Fe most likely occurs in the form of small particles and colloids of amorphous Fe oxyhydroxides (eg. ferrihydrite) (Campbell, 1991). Thus, unlike upwelled $\mathrm{N}$ and $\mathrm{P}$, the bioavailability of the upwelled $\mathrm{Fe}$ in the HNLC equatorial Pacific is poorly known. The bioavailability of new $\mathrm{Fe}$ inputs are also poorly known in other remote ocean areas such as the subtropical gyres, where new Fe is thought to be added in the form of aeolian dust (Martin and Fitzwater, 1988). Heterotrophic protists could potentially play a significant role in making exogenous mineral iron bioavailable by ingesting small mineral particles and colloids and facilitating their dissolution via intracellular digestion. 


\section{Contextual synthesis and motivation for thesis research}

As described in the preceding sections of this chapter, heterotrophic protists in the nanoplanktonic size class are potentially an important influence on the marine geochemistry of trace metals. Small protozoan grazers are the primary sink for a variety of particles in the $0.1-1 \mu \mathrm{m}$ size class in marine systems, and such particles are known to constitute a major reservoir of particle reactive trace metals. Through intracellular digestion protists can cause changes in the redox state and coordination chemistry of ingested trace metals. These changes can have significant impacts on trace metal cycling in the marine environment. Tracer studies indicate that protozoan grazers are capable of regenerating trace metals from ingested prey to the dissolved phase, and in some cases these regenerated trace metals appear to be available for biological uptake. Ecological studies indicate that, at least in HNLC regions, recycling of $\mathrm{Fe}$ as mediated by microzooplankton grazers may be a key parameter in ecosystem productivity. Thus the available evidence suggests that the effect of protozoan grazing on trace metals in the oceans is both geochemically and ecologically significant.

Despite this evidence, there has not yet been any systematic investigation of grazermediated effects on trace metal speciation and partitioning in seawater. This thesis began with the overall objective of conducting such a study, motivated by the desire to understand the basic mechanism(s) by which grazers influence trace metals in the marine environment. The emphasis in this research has been on the use of model systems to investigate how protozoan grazing transforms strongly hydrolyzed, particle reactive trace metals and/or the solid phase with which they are associated, and to determine how these transformations may influence the fate of the trace metals in the system. Because of its general focus on grazer/solid phase/trace metal interaction, this work is relevant not only to trace metal cycling in open ocean systems; but also to the cycling of particle reactive trace metals (and particle reactive contaminants) in coastal and estuarine environments. The final section of this introductory chapter will briefly describe how the rest of the thesis eventually developed, given the basic motivation and objectives described above. 


\section{Thesis - structure and development}

Chapter 2 presents the results of a series of experiments which focus on grazermediated transformations of particle reactive trace metals associated with bacteria cells. These initial experiments demonstrated the potential for protozoan grazers to affect the speciation and partitioning of strongly hydrolyzed trace metals associated with organic phases. In addition, this research served to delineate the basic behavior of the model systems and organisms employed throughout this research. In the next chapter, an additional element was added to the model systems: colloidal iron oxides.

Chapter 3 presents the initial results of an investigation into the effect of protozoan ingestion/digestion on colloidal ferrihydrite, a commonly-occurring iron oxide. This investigation revealed that significant dissolution of colloidal ferrihydrite under oxic conditions can occur within the microenvironment of the phagotrophic food vacuole of heterotrophic protists. Ferrihydrite is an abundant form of iron, a significant carrier phase for a variety of chemical species, and a participant in many environmentally relevant redox reactions. Few mechanisms for the dissolution of iron oxides have been identified in oxic environments, other than thermal, ligand-mediated, or photoreductive dissolution. Thus, the finding of a novel dissolution pathway via intracellular digestion by protists was taken as fairly significant, and dictated the direction of the remainder of the thesis.

Having demonstrated the existence of a protozoan-mediated dissolution mechanism in Chapter 3, the next objective was to determine its significance in the natural environment relative to other known dissolution mechanisms, particularly photoreduction. In order to study the dissolution of iron oxides by protozoan grazers in more dilute natural systems, a novel method of measuring the dissolution rate of synthetic iron oxides in seawater had to be devised. This technique had to rely not on detecting changes in iron oxide reactivity, as in previous methods, but rather on an absolute indication of the extent of dissolution. Chapter 4 presents the development of this method, which is based on the synthesis of ferrihydrite impregnated with an inert tracer. Initial applications of this method to the study of iron oxide dissolution by a variety of mechanisms are also presented. 
Chapter 5 presents the application of the inert tracer method to the measurement of the absolute dissolution rate of colloidal ferrihydrite as mediated by protozoan grazers. A number of experiments utilizing this technique are presented, both in the laboratory using cultures of protozoan grazers, and in the field using natural consortia from Vineyard Sound, MA. Quantitative estimates of the significance of grazer-mediated dissolution of colloidal iron oxide relative to photoreductive dissolution are presented.

Chapter 6 summarizes the thesis as a whole, detailing the principal results and contributions made throughout. A discussion follows of the wider implications of this work, both with regard to the impact of protists on trace metal cycling in the marine environment, and with regard to phagotrophy as a vector for trace metal/biota interaction in aquatic systems. Chapter 6 concludes with suggestions for further research on these and related topics. 


\section{References}

- Adey, W.H. and K. Loveland. 1991. Dynamic Aquaria. p. 465. Academic Press, Inc. San Diego, CA.

- Allen, R.D. and A.K. Fok. 1984. Stages of digestive vacuoles in Paramecium: membrane surface differences and location. Eur. J. Cell Biol. 35: 149-155.

- Andersen, O.K., J.C. Goldman, D.A. Caron and M.R. Dennett. 1986. Nutrient cycling in a microflagellate food chain: III. Phosphorous dynamics. Mar. Ecol. Prog. Ser. 31: 4755 .

- Azam, F., T. Fenchel, J.G. Field, L.A. Meyer-Reil and F. Thingstad. 1983. The ecological role of water-column microbes in the sea. Mar. Ecol. Prog. Ser. 10: 257-263.

- Bacon, M.P. and R.A. Anderson. 1982. Distribution of the thorium isotopes between dissolved and particulate forms in the deep sea. J. Geophys. Res. 87: 2045-2056.

- Balistrieri, L.S., P.G. Brewer and J.W. Murray. 1981. Scavenging residence times of trace metals and surface chemistry of sinking particles in the deep ocean. Deep-Sea Res. 28: $101-121$.

- Baskaran, M. and P.H. Santschi. 1993. The role of particles and colloids in the transport of radionuclides in coastal environments of Texas. Mar. Chem. 43: 95-114.

- Boyle, E.A., S.D. Chapnick, G.T. Shen and M.P. Bacon 1986. Temporal variability of lead in the western North Atlantic. J. Geophys. Res. 91: 8573-8593.

- Bruland, K.W. 1983. Trace elements in seawater. p. 157-220. In Chemical Oceanography, Vol. 8. Riley, J.P and R. Chester, eds. Academic Press, London.

- Bruland, K.W., J.R. Donat and D.A.Hutchins. 1991. Interactive influences of bioactive trace metals on biological production in oceanic waters. Limnol. Oceanogr. 36:15551577.

- Campbell, A.C. 1991. Mineralogy and chemistry of particles by synchrotron X-ray, Mossbauer and plasma MS. p. 375-390. In Marine Particles: Analysis and Characterization. Hurd, D.C. and D.W. Spencer, eds. Geophysical Monograph 63. AGU, Washington, D.C. 
- Caron, D.A. 1990. Evolving role of protozoa in aquatic nutrient cycles. p. 387-416. In Protozoa and their Role in Marine Processes, NATO ASI Series, Vol. G 25. Reid, P.C., C.M. Turley and P.H. Burkhill, eds. Springer-Verlag Berlin Heidelberg.

- Caron, D.A., J.C. Goldman and M.R. Dennett. 1988. Experimental demonstration of the roles of bacteria and bacterivorous protozoa in plankton nutrient cycles. Hydrobiologia 159: $27-40$.

- Caron, D.A., J.C. Goldman, O.K. Andersen and M.R. Dennett.1985. Nutrient cycling in a microflagellate food chain: II. Population dynamics and carbon cycling. Mar. Ecol. Prog. Ser. 24: 243-254.

- Chase, Z. and N.M. Price. 1998. Metabolic consequences of iron deficiency in heterotrophic marine protozoa. Limnol. Oceanogr. in press.

- Cho, B.C. and F. Azam. 1990. Major role of bacteria in biogeochemical fluxes in the ocean's interior. Nature 332: 441-443.

- Coale, K.H and K.W. Bruland. 1985. ${ }^{234} \mathrm{Th}:{ }^{238} \mathrm{U}$ disequilibria within the California current. Limnol. Oceanogr. 30:22-33.

- Coale, K.H. and K. W. Bruland. 1987. Oceanic stratified euphotic zone as elucidated by ${ }^{234} \mathrm{Th}:{ }^{238} \mathrm{U}$ disequilibria. Limnol. Oceanogr. 32: 189-200.

- Coale, K.H., S.E. Fitzwater, R.M. Gordon, K.S. Johnson and R.T. Barber. 1996. Control of community growth and export production by upwelled iron in the equatorial Pacific Ocean. Nature 379: 621-624.

- Fenchel, T. 1987. Ecology of Protozoa: The Biology of Free-living Phagotrophic Protists. Madison: Science Tech Publishers.

- Fischer-Defoy, D. and K. Hausmann. 1982. Ultrastructural characteristics of algal digestion by Climacostomum virens (Ciliata) (Ehrenberg) Stein. Zoomorph. 100: 121130 .

- Fisher, N.S., C.V. Nolan and S.W. Fowler. 1991. Assimilation of metals in marine copepods and its biogeochemical implications. Mar. Ecol. Prog. Ser. 71: 37-43. 
- Fok, A.K., J.H. Muraoka and R.D. Allen. 1984. Acid phosphatase in the digestive vacuoles and lysosomes of Paramecium caudatum: A timed study. J. Protozool. 31: 216-220.

- Fok, A.K., M.S. Ueno and E.A. Azada. 1985. Rapid and sensitive assays for phagosomal acidification in Paramecium and Tetrahymena. Eur. J. Cell Biol. 38: 306311.

- Fok, A.K., Y. Lee and R.D. Allen. 1982. The correlation of digestive vacuole $\mathrm{pH}$ and size with the digestive cycle in Parmecium caudatum. J. Protozool. 29: 409-414.

- Fowler, S.W. 1977. Trace elements in zooplankton particulate products. Nature 269: $51-53$.

- Frey, B.E. and L.F. Small. 1979. Recycling of metabolized iron by the marine dinoflagellate Amphidinium carterae. J. Phycol. 15: 405-409.

- Goldman, J.C. 1984. Conceptual role for microaggregates in pelagic waters. Bull. Mar. Sci. 35: 462-476.

- Goldman, J.C., D.A. Caron and M.R. Dennett. 1987. Nutrient cycling in a microflagellate food chain: IV. Phytoplankton-microflagellate interactions. Mar. Ecol. Prog. Ser. 38: 75-87.

- Goldman, J.C., D.A. Caron, O.K. Andersen and M.R. Dennett. 1985. Nutrient cycling in a microflagellate food chain: I. Nitrogen dynamics. Mar. Ecol. Prog. Ser. 24: 231-242.

- Gordon, R.M., K.H. Coale and K.S. Johnson. 1997. Iron distributions in the equatorial Pacific: Implications for new production. Limnol. Oceanogr. 42: 419-431.

- Hagstrom, A. and U. Larsson. 1984. Diel and seasonal variation in growth rates of pelagic bacteria. p. 29-262. In Heterotrophic Activity in the Sea. Hobbie, J.E. and P.J. LeB. Williams, eds. Plenum Press, NY.

- Honeyman, B.D. 1991. Surface chemistry, colloids and trace-element scavenging. p.437-451. In Marine Particles: Analysis and Characterization. Hurd, D.C. and D.W. Spencer, eds. Geophysical Monograph 63. AGU, Washington, D.C.

- Honeyman, B.D. and P.H. Santschi. 1989. A Brownian-pumping model for oceanic trace metal scavenging: Evidence from Th isotopes. J. Mar. Res. 47: 951-992. 
- Hudson, R.J.M. and F.M.M. Morel. 1990. Iron transport in marine phytoplankton: Kinetics of cellular and medium coordination reactions. Limnol. Oceanogr. 35: 10021020.

- Hutchins, D.A. and K.W. Bruland. 1994. Grazer-mediated regeneration and assimilation of Fe, $\mathrm{Zn}$ and Mn from planktonic prey. Mar. Ecol. Prog. Ser. 110: 259-269.

- Hutchins, D.A. and K.W. Bruland. 1995. Fe, Zn, Mn and N transfer between size classes in a coastal phytoplankton community: Trace metal and major nutrient recycling. J. Mar. Res. 53: 297-313.

- Hutchins, D.A., G.R. DiTullio and K.W. Bruland. 1993. Iron and regenerated production: Evidence for biological iron recycling in two marine environments. Limnol. Oceanogr. 38: 1242-1255.

- Ishida, M., M.S. Aihara, R.D. Allen and A.K. Fok. 1997. Acidification of the young phagosomes of Paramecium is mediated by proton pumps derived from the acidisomes. Protoplasma 196: 12-20.

- Jackson, G.A. 1980. Phytoplankton growth and zooplankton grazing in oligotrophic oceans. Nature, Lond. 284: 439-441.

- Landry, M.R. and others. 1997. Iron and grazing constraints on primary production in the central equatorial Pacific: An EqPac synthesis. Limnol. Oceanogr. 42: 405-418. - Lee, T., E. Barg, D. Lal and F. Azam. 1993. Bacterial scavenging of ${ }^{234}$ Th in surface ocean waters. Mar. Ecol. Prog. Ser. 96: 109-116.

- Martin J.H. and S.E. Fitzwater. 1988. Iron deficiency limits phtoplankton growth in the north-east Pacific subarctic. Nature. 331:341-343.

- Mellman, I., R. Fuchs and A. Helenius. 1986. Acidification of the endocytic and exocytic pathways. Ann. Rev. Biochem. 55: 663-700.

- Moran, S.B. and Buesseler, K.O. 1992. Short residence time of colloids in the upper ocean estimated from ${ }^{234} \mathrm{Th}{ }^{238} \mathrm{U}$ disequilibria. Nature 359: 221-223.

- Murray, J.W., J.N. Downs, S. Strom, C. Wei and H.W. Jannasch. 1989. Nutrient assimilation, export production and ${ }^{234} \mathrm{Th}$ scavenging in the eastern equatorial Pacific. Deep-Sea Res. 36: 1471-1489. 
- Nagata, T. and D.L. Kirchman. 1992. Release of macromolecular organic complexes by heterotrophic marine flagellates. Mar. Ecol. Prog. Ser. 83: 233-240.

- Neilands, J.B. 1981. Iron absorption and transport in microorganisms. Ann. Rev. Nutr. 1: 27-46.

- Nozaki, Y., H.-S. Yang and M. Yamada. 1987. Scavenging of thorium in the ocean. Geophys. Res. 87: 2045-2056.

- Peck, R.K. and K. Hausmann. 1980. Primary lysosomes of the ciliate Pseudomicrothorax dubius: Cytochemical identification and role in phagocytosis. $J$. Protozool. 27: 401-409.

- Pomeroy, L.R. 1974. The ocean's food web, a changing paradigm. Bioscience 24: 499504.

- Pond, D.W., R.P. Harris and C. Brownlee. 1995. A microinjection technique using a $\mathrm{pH}$-sensitive dye to determine the gut $\mathrm{pH}$ of Calanus helgolandicus. Mar. Biol. 123: 7579.

- Proctor, L.M. and J.A. Fuhrman. 1990. Viral mortality of marine bacteria and cyanobacteria. Nature 343: 60-62.

- Reinfelder, J.R. and N.S. Fisher. 1991. The assimilation of elements ingested by marine copepods. Science 251: 794-796.

- Schindler, P.W. 1975. Removal of trace metals from the ocean: a zero order model. Thalassia. Jugosl. 11: 101-111.

- Sherr, E.B., B.F. Sherr, R.D. Fallon and S.Y. Newell. 1986. Small, aloricate ciliates as a major component of the marine heterotrophic nanoplankton. Limnol. Oceanogr.31:177183.

- Sieburth, J.McN. 1984. Protozoan bacterivory in pelagic marine waters. p. 405-444. In Heterotrophic Activity in the Sea. Hobbie, J.E. and P.J.LeB. Williams, eds. Plenum Press, NY.

- Small, L.F. and S.W. Fowler. 1973. Turnover and vertical transport of zinc by the euphausid Meganyctiphanes norvegica in the Ligurian Sea. Mar. Biol. 18: 284-290. - Stossel, T.P. 1974. Phagocytosis [first of 3parts]. N. Engl. J. Med. 290: 717-723. 
- Stramski, D., F. Rassoulzadegan and D.A. Keifer. 1992. Changes in the optical properties of a particle suspension caused by protist grazing. J. of Plank. Res. 14: 961977.

- Sunda, W.G. 1988-1989. Trace metal interactions with marine phytoplankton. Biol. Oceanogr. 6: 411-442.

- Tortell, P.D., M.T. Maldonado and N.M. Price. 1996. The importance of heterotrophic bacteria in Fe-limited ocean ecosystems. Nature 383: 330-332.

- Tranvik, L. 1994. Colloidal and dissolved organic matter excreted by a mixotrophic flagellate during bacterivory and autotrophy. Appl. Env. Microbiol. 60: 1884-1888.

- Twiss, M.R. and P.G.C. Campbell. 1995. Regeneration of trace metals from picoplankton by nanoflagellate grazing. Limnol. Oceanogr. 40: 1418-1429.

- Twiss, M.R., P.G.C. Campbell and J.-C. Auclair. 1996. Regeneration, recycling and trophic transfer of trace metals by microbial food-web organisms in the pelagic surface waters of Lake Erie. Limnol. Oceanogr. 41: 1425-1437.

- Valiela, I. 1995. Marine Ecological Processes. Springer, New York.

- Vonk, H.J., 1960. Digestion and metabolism. p.291-313. In The Physiology of Crustacea. Waterman, T.H. ed. Academic Press, New York and London. 


\section{CHAPTER 2}

\section{Remineralization and recycling of particle-reactive trace metals by protozoan grazers: Fate of cell-associated metals}

\section{Introduction}

\section{General Background}

The geochemical cycling of particle-reactive trace metals in the oceans is, of course, intimately tied to the cycling of particles. Particle production, sinking, and degradation are processes driven primarily by biota. Phytoplankton produce particles in surface waters, which take up metals either actively (as nutrients) or passively (scavenging). These particles are consumed by grazers and repackaged into fecal pellets or grazer biomass. Fecal pellets sink out of the euphotic zone fairly rapidly, transporting associated trace metals into the oceanic interior. There, microbial degradation of organic material can result in the regeneration of trace metals into the water column (Bruland, 1983).

This classical paradigm of the role of biology in trace metal cycling, dominated by interactions between phytoplankton, copepods, and bacteria, is based on an equally classical paradigm of the pelagic ecosystem: Phytoplankton uptake of inorganic nutrients, consumption of phytoplankton by metazoan grazers, transfer from there to higher trophic levels and degradation of wastes by bacteria (Riley, 1963). In the past 20 years, our concept of this ecosystem has changed a great deal due to the emergence of a new paradigm, that of the microbial loop. This model of the marine food web, based on the consumption of significant amounts of primary production by bacteria and the consumption of bacterial biomass by protozoan grazers, has dramatically changed our view of the trophic relationships and cycling of nutrients within the planktonic ecosystem (Pomeroy, 1974; Azam et. al., 1983; Sherr and Sherr, 1988).

Just as the emergence of the microbial loop has altered our concept of nutrient cycling in the upper oceans, so it is now changing our understanding of trace element cycling. The role of the microbial loop in the marine geochemistry of trace elements can be 
considered in light of the fact that particles in the bacterial size class $(0.1-1 \mu \mathrm{m})$ constitute the largest reservoir of biological surface area (and by extension the largest pool of biotically-associated surface-reactive trace elements) in the ocean (Azam et. al., 1992). The fate of most of this bacterial biomass, and of the trace metals associated with it, is to be consumed by heterotrophic protists (Goldman, 1984; Sieburth, 1984). These grazers, therefore, must play a significant role in the cycling of trace metals in the oceans. Because protists occur ubiquitously in the marine environment, (Fenchel, 1987), their influence is relevant not only to trace metals in the open ocean, but also to the fate and transport of particle-reactive metals and pollutants in coastal environments, estuarine systems, and at the sediment-water interface. Protists can affect the mobility of particleassociated contaminants as a consequence of both the physical process of particle ingestion and the chemical process of particle digestion.

The experiments described in this chapter are an attempt to evaluate the potential for protozoan grazers to affect the cycling of trace metals in seawater, in particular particlereactive trace metals. This research is motivated by some basic questions. Given a particle-reactive trace element associated with a bacterial cell, what will be the fate of this element upon consumption of the bacteria by a protozoan grazer? Will the metal accumulate in grazer biomass? Will it instead be solubilized by the digestive action of the grazer and released into the dissolved phase? Is the metal in this case truly dissolved, or rather associated with colloidal material? Does the metal actually get ejected from the protist as part of solid waste material? Do the surface active properties of this material induce aggregation, causing the metal to end up in a larger particle size class than it came from originally? How do these effects differ between different metals, and between different grazers? The experiments in this chapter provide insight into the answers to these questions, and contribute to our knowledge of how heterotrophic protists influence the geochemistry of trace metals in the marine environment. 


\section{Selection of trace metals}

Previous studies on the role of protozoan grazers in regenerating cell-associated trace metals have focused primarily on intracellular trace metal micronutrients (eg. Hutchins and Bruland, 1994; Twiss et. al., 1996). As micronutrients, the behavior of trace metals in response to grazer ingestion may be expected to be similar to that of the major nutrients, nitrogen and phosphorus, known to be efficiently remineralized from bacterial biomass through the action of protozoan grazers. This work focuses on trace metals ( $\mathrm{Th}, \mathrm{Fe}, \mathrm{Cr}$ ) whose chemistry, at least in the model systems employed here, is strongly influenced by hydrolysis reactions and surface adsorption. These metals, because of their particle reactivity, are likely to exhibit fairly complex solid/solution partitioning behavior in response to protozoan grazing. In addition, because these metals are normally strongly hydrolyzed at seawater $\mathrm{pH}$, they could undergo a dramatic change in speciation upon passage through the acidic food vacuole of a phagotrophic protist (Fok et. al., 1992).

Th is often used in oceanographic studies as a general analogue for particle-reactive trace metals (Clegg and Sarmiento, 1989; Clegg and Whitfield, 1991). This is due not only to Th chemistry in seawater (Th occurs as a +4 cation, strongly hydrolyzed and very particle reactive) but also to the convenience of Th isotopes (produced as part of the uranium decay series) as in-situ radiotracers. ${ }^{234} \mathrm{Th}$, for example, has been widely applied in recent years as a tracer for the export of organic carbon out of the euphotic zone (Buesseler et. al., 1992, 1995; Murray et. al. 1996). This isotope has also recently been applied in studies of the cycling of hydrophobic organic contaminants in the marine environment (Gustafsson et. al, 1997). Because Th isotopes are so frequently used as tracers of these kinds of processes, a study of how Th is cycled through the microbial loop could significantly contribute to a wide range of oceanographic research. ${ }^{234} \mathrm{Th}$, a gamma-emitting radio-isotope, was therefore chosen as one of the tracers in the experiments described in this chapter.

The idea that primary production in some areas of the world's oceans could be limited by iron was brought to prominence by John Martin in the 1980's (Martin and Fitzwater, 1988). Since that time, a great deal of research in chemical and biological oceanography 
has been devoted to studies of the marine chemistry of iron and the factors which affect its availability to phytoplankton (Wells et. al., 1995). Recent studies in the Equatorial Pacific, a region where primary productivity is thought to be limited by iron, have indicated that the efficient recycling of iron though the microbial loop is a key process in the biogeochemistry of iron in this environment (Landry et. al., 1997). Only a very few laboratory studies have examined the mechanistics of iron recycling in seawater via protozoan grazing (Hutchins and Bruland, 1994; Chase and Price, 1998). Both of these studies employed the organism Paraphysomonas sp. To expand upon previous results by comparison with additional organisms and to examine the regeneration of iron in seawater without synthetic chelators, ${ }^{59} \mathrm{Fe}$ was used to trace the cycling of iron in the grazing experiments described here.

The final tracer used in these experiments was ${ }^{51} \mathrm{Cr}$, as $\mathrm{Cr}(\mathrm{IIII}) . \mathrm{Cr}(\mathrm{III})$ is a known pollutant in some estuaries (eg. Saco River Estuary, ME; Salem Harbor, MA) and can be highly toxic (Decho and Luoma, 1994). Concerns about chromium toxicity usually center around $\mathrm{Cr}(\mathrm{VI})$, the more oxidized form of chromium in aquatic systems. $\mathrm{Cr}(\mathrm{VI})$ occurs as an oxyanion and as such is highly mobile and can permeate cell membranes (Nieboer and Jusys, 1988). Cr(III) is strongly hydrolyzed and very particle reactive. It can form complexes with a variety of organic ligands, and the ligand exchange kinetics of these complexes are exceedingly slow (Margerum et. al., 1978; Nieboer and Jusys, 1988). Because of this, it is generally thought of as non-toxic and permanently associated with sediments and other particles in estuaries. $\mathrm{Cr}$ (III) has been used as an inert tracer in carbon assimilation studies, because it was not thought to be bioavailable (Mertz, 1969; Lopez et. al., 1989). Subsequent work has shown that bivalves can absorb Cr(III) from certain food particles as a consequence of intracellular digestion (Decho and Luoma, 1991). Phagotrophic protozoans have digestive processes very similar to intracellular digestion in bivalves, and so ${ }^{51} \mathrm{Cr}$ as $\mathrm{Cr}(\mathrm{III})$ was employed in our studies in order to determine whether it could be mobilized from bacterial cells due to digestion by protists.

Although each metal used in this study is of some interest in and of itself, examining the combined behavior of all three together can also provide insight into the cycling of 
particle-associated trace elements via the microbial loop. Th, for example, is associated primarily with bacterial surfaces because it is passively scavenged. Iron, in contrast, occurs mainly as part of the intracellular pool because it is actively taken up. Comparing the behavior of these two elements can therefore provide information concerning the fate of intracellular vs. extracellular trace elements upon consumption of bacterial biomass by protozoans. It is also of interest to compare $\mathrm{Cr}$ (III) with $\mathrm{Fe}$ (III). The coordination chemistry of these two tri-valent cations is practically identical, and their chemical behavior is quite similar except that $\mathrm{Cr}$ (III) has much slower ligand exchange kinetics than Fe(III) (Nieboer and Jusys, 1988). Differences in the behavior of $\mathrm{Cr}(\mathrm{III})$ and $\mathrm{Fe}(\mathrm{III})$ may thus be related to the kinetics of ligand exchange.

\section{$\underline{\text { Study organisms }}$}

Heterotrophic protozoans in the marine environment are a diverse group, consisting of a variety of flagellated, amoeboid, and ciliated forms (Lee and Capriulo, 1990). These organisms can differ significantly in terms of their morphology, metabolism, and specific ecological niche. All of these factors could contribute to differences between protozoan species with regard to the effect of grazing on trace metal chemistry. In order to get a sense of the possible degree of variation, several different protozoan species were used in the experiments presented here. These organisms were taken from the culture collection of David Caron, and were chosen as being representative of organisms commonly found in a variety of marine habitats. The grazers involved in the experiments described in this chapter include the following:

Uronema sp. (clone BBcil) - a small scuticociliate, $7-15 \mu \mathrm{m}$ in length, isolated from Buzzard's Bay, MA. These ciliates are filter feeders, using their cilia to generate water currents that sweep food particles into their cytostomes (oral area). As filter feeders, they retain all particles above a certain size. They are rapid and efficient feeders, having the highest clearance rates of the three protists described here.

Paraphysomonas imperforata (clone VS1) - a chrysomonad flagellate isolated from Vineyard Sound, $5-7 \mu \mathrm{m}$ in diameter. This organism is something of a cross between a 
filter feeder and a raptorial feeder, in that it propels itself through the water behind its flagellum, creating a current that sweeps food towards the cell. Paraphysomonas has the lowest clearance rate of these three protists.

Cafeteria sp. (clone Cflag) - a bicosoecid flagellate isolated from a seawater aquarium, 3$5 \mu \mathrm{m}$ in diameter. These organisms are true raptorial feeders, swimming with the flagellum behind and intercepting particles in front. As a raptorial feeder, Cafeteria may be expected to exhibit some selectivity for food particles based on characteristics other than size.

In all experiments and in maintenance cultures, the prey organism used was the marine bacterium Halomonas halodurans (previously identified as Pseudomonas halodurans). This organism grows well in culture, provides a good food source for all of the protists, and is fairly representative of heterotrophic marine bacteria.

\section{Basic experimental protocol}

All experiments were performed in simple one- and two-member model systems. The protocol for setting up and sampling these model systems was basically the same in all cases. Setting up model systems involved first growing up the bacterial prey, $H$. halodurans, in $0.2 \mu \mathrm{m}$ filtered, sterilized Vineyard Sound water (VSW) with yeast extract added as growth substrate. Cultures were grown to late exponential or lag phase and then harvested by centrifugation. Bacteria were then resuspended at a more dilute concentration. At this point $\left(\mathrm{t}_{0}\right)$ half of the bacterial suspensions were inoculated with an aliquot from a protistan culture. Starting at $t_{0}$, the uninoculated bacterial suspensions (control) and the inoculated suspensions (experimental) were sampled every 10-12 hours for various parameters. Sampling continued until grazers had reduced the concentration of bacteria below the efficient grazing threshold (about $10^{6} \mathrm{cells} / \mathrm{ml}$ ). More specific details regarding model system set-up and sampling for each series of experiments are included in the experimental section that follows. 


\section{Overview of experiments}

The experimental section that follows is divided into two main sections, series \#1 and series \#2. Series \#1 was a sequence of three experiments involving the grazers Uronema, Cafeteria, and Paraphysomonas. These experiments were preliminary, their purpose being to delineate the basic behavior of the model systems on the whole and the general extent of variation between protozoan species.

Series \#2 was a sequence of two experiments involving only Cafeteria and Paraphysomonas. These experiments were designed to be more definitive, with set-up and sampling protocol based on the results of series \#1. Results and discussion of both series \#1 and series \#2 are included in the experimental section in order to demonstrate the progression between the two series and the relationships between the data. The conclusion contains a brief summary of results, as well as plans for further experimental work.

\section{Experimental}

\section{Series \#1 - Initial survey}

\section{Method}

Radio-isotopes $-{ }^{59} \mathrm{Fe}$ as $\mathrm{Fe}(\mathrm{III})$ and ${ }^{51} \mathrm{Cr}$ as $\mathrm{Cr}(\mathrm{III})$ were ordered from New England Nuclear as chloride salts in dilute $(0.5 \mathrm{~N}) \mathrm{HCl}$. Stocks were diluted and added to bacteria labeling cultures to result in a final activity level in control and experimental cultures of about $2 \mu \mathrm{Ci}$ per liter.

${ }^{234} \mathrm{Th}$ was extracted from a solution of $0.5 \mathrm{M}$ uranyl nitrate in $3 \mathrm{M}$ perchloric acid, following an extraction procedure provided by Alan Fleer (WHOI). ${ }^{234} \mathrm{Th}$ was concentrated from the uranyl nitrate solution onto a column of Biorad 100-200 mesh AG50W-X8 cation exchange resin ( $\mathrm{H}^{+}$form); the column was washed with several small volumes of $3 \mathrm{M} \mathrm{HCl}$; and ${ }^{234} \mathrm{Th}$ was selectively eluted with $0.5 \mathrm{M}$ oxalic acid. Concentrated nitric and perchloric acid were added to the eluate, which was then evaporated down to perchloric acid fumes. The ${ }^{234} \mathrm{Th}$ was then resuspended in $3 \mathrm{M}$ perchloric acid. The concentration/elution procedure was repeated twice to ensure a 
clean separation of ${ }^{234} \mathrm{Th}$. For each of the three experiments in series \#1, ${ }^{234} \mathrm{Th}$ was extracted from about $50 \mathrm{~g}$ of uranyl nitrate.

Set-up - Radiolabeled bacteria (H. halodurans) for experiments were grown up in $0.2 \mu \mathrm{m}$ filtered, sterilized Vineyard Sound seawater (VSW) with $0.06 \%$ yeast extract added as a substrate. VSW was inoculated with bacteria from an agar slant culture, and radio-tracers $\left({ }^{59} \mathrm{Fe},{ }^{234} \mathrm{Th},{ }^{51} \mathrm{Cr}\right)$ were then added. The acidity of the radiotracer spikes was neutralized by adding $1 \% \mathrm{KOH}$ solution to the VSW. The bacteria cultures were grown in acidwashed polypropylene bottles with substantial headspace, on a shaker under normal laboratory fluorescent lighting. Bacteria were harvested after three days, at which time the cultures were in late exponential to stationary phase. Harvesting was performed by centrifugation. Cells were spun down and resuspended three times in VSW to remove excess yeast extract and radio-tracers in the dissolved phase. The bacterial cell concentrates were then resuspended in 1.5 liters of VSW in 2.5 liter polycarbonate bottles. This dilution was approximately $1 / 10$, resulting in a final bacterial cell concentration in each bottle of about $3-5 \times 10^{7}$ cells $/ \mathrm{ml}$.

At $t_{0}$, one of the bottles of bacterial suspension was inoculated with $10 \mathrm{ml}$ of a protozoan grazer culture in exponential growth. (This was achieved by starting the grazer culture for inoculation 2-3 days earlier, on a culture of bacteria grown in VSW with $0.03 \%$ yeast extract.) The bacterial suspension inoculated with protists is hereafter referred to as the experimental bottle, and the uninoculated bacterial suspension as the control. Each of the three experiments in series \#1 had only one experimental bottle and one control (i.e. no replicates). Experimental and control bottles were incubated at room temperature, without shaking, for the duration of the experiment.

Sampling and Analysis - Starting from $\mathrm{t}_{0}$ and continuing for 60 hours, size-fractionated samples were taken every 10 hours from both control and experimental cultures. Cultures were gently mixed prior to sampling by swirling and/or tipping the bottles. Size fractionation was performed by non-sequential filtration, using $25 \mathrm{~mm}$ diameter 
Nuclepore polycarbonate filters of $0.2 \mu \mathrm{m}, 1 \mu \mathrm{m}$, and 5 (Cafeteria and Paraphysomonas) or $10 \mu \mathrm{m}$ (Uronema) pore size. Volumes passed through the filters were as follows: $10 \mathrm{ml}$ through the $0.2 \mu \mathrm{m}$ filter; $25-50 \mathrm{ml}$ through the $1 \mu \mathrm{m}$ filter; $100 \mathrm{ml}$ through the 5 and 10 $\mu \mathrm{m}$ filters. Filtration was performed under gentle vacuum $(<100 \mathrm{~mm} \mathrm{Hg})$, and filters were not rinsed. Filtrates were discarded. The $1-0.2 \mu \mathrm{m}$ size fraction contained the bacteria, and the 1-5 or 1-10 $\mu \mathrm{m}$ size fractions contained most of the grazers. (Confirmed by microscopy.) Size fractions were obtained by difference. At each time point, an unfiltered aliquot was taken as a total to which all particulate fractions were normalized, thereby correcting for any wall loss of tracers (which was about $10 \%$ at the most). Filters and totals were counted for gamma activity on a Canberra low-energy Germanium detector coupled to an Ortec multi-channel analyzer. All isotopes were decay-corrected to the time of sampling. All samples were counted for a length of time sufficient to reduce statistical counting errors to $5 \%$ or less.

\section{$\underline{\text { Results }}$}

Results of the first experiment in the series, with Uronema as the grazer, are shown for both ${ }^{59} \mathrm{Fe}$ (Fig. 2.1) and ${ }^{234} \mathrm{Th}$ (Fig. 2.2) size fractionation. For ${ }^{59} \mathrm{Fe}$ in the control culture, activity was almost entirely limited to the $0.2-1 \mu \mathrm{m}$ size class, which contained the bacteria. The action of the grazer resulted in removal of the ${ }^{59} \mathrm{Fe}$ from the bacterial size class, and its redistribution between the $<0.2 \mu \mathrm{m}$ and $1-10 \mu \mathrm{m}$ size class. The behavior of ${ }^{234} \mathrm{Th}$ was fairly similar to ${ }^{59} \mathrm{Fe}$, with some key differences. In the control, there was desorption of $\mathrm{Th}$ from the $0.2-1 \mu \mathrm{m}$ particles, resulting in a distribution split fairly evenly between the dissolved and the solid phase. Similarly to ${ }^{59} \mathrm{Fe}$, the action of the grazer removed ${ }^{234} \mathrm{Th}$ from the bacterial size class. ${ }^{234} \mathrm{Th}$ exhibited a somewhat greater tendency than ${ }^{59} \mathrm{Fe}$ to go into the dissolved phase as a result of grazing, and there was a smaller accumulation of ${ }^{234} \mathrm{Th}$ in the $1-10 \mu \mathrm{m}$ size class.

The results of the next grazing experiment, with Paraphysomonas, included an additional radionuclide, ${ }^{51} \mathrm{Cr}$ (Figs. 2.3-2.5). The controls for ${ }^{59} \mathrm{Fe}$ and ${ }^{234} \mathrm{Th}$ in this experiment were similar to the first, except that there was no desorption of ${ }^{234} \mathrm{Th}$ from the 

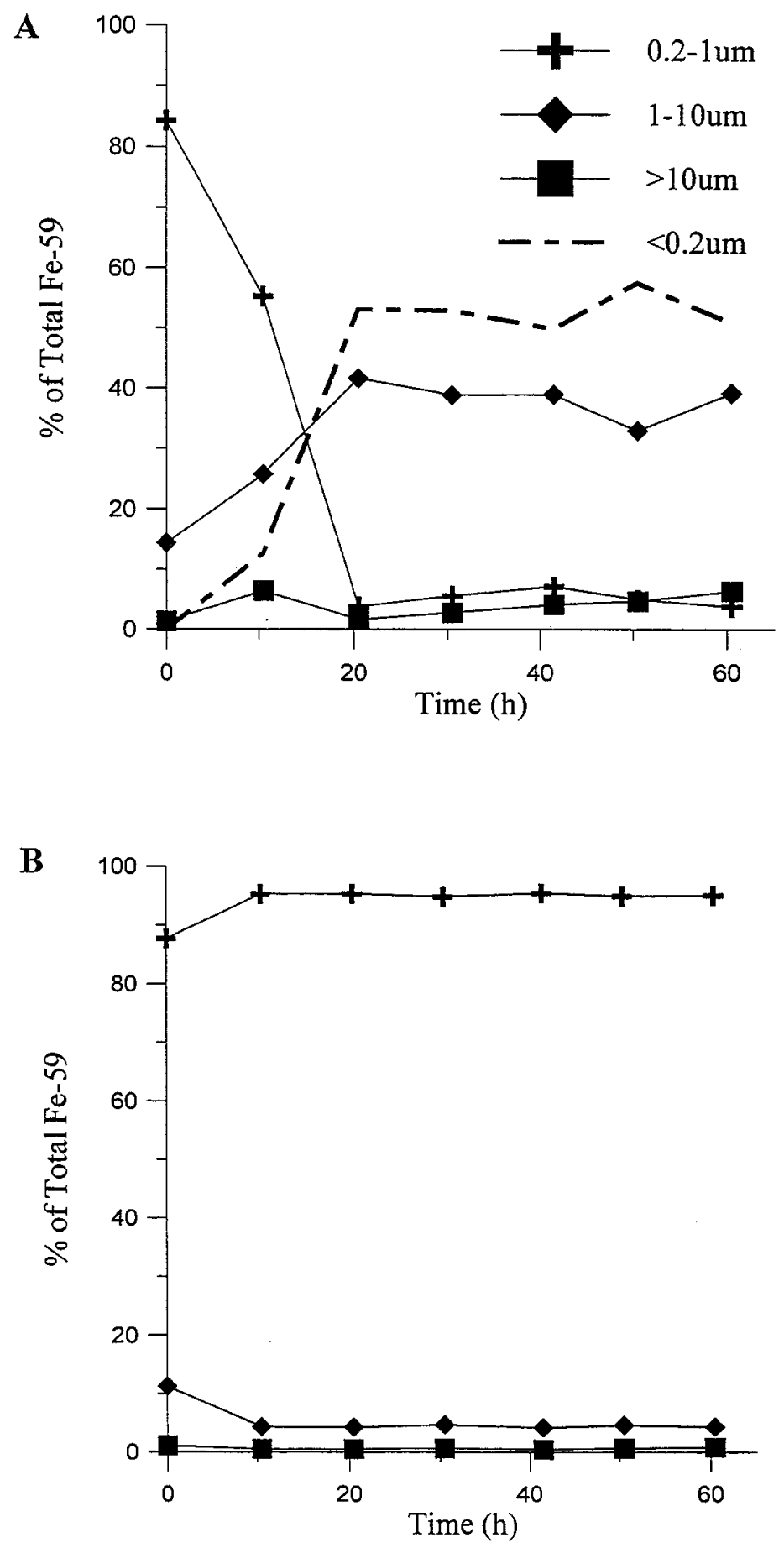

Figure 2.1 - Experimental series \#1, Uronema experiment.

$\%$ of total Fe-59 activity in various size fractions vs.time

A: Grazing culture; B: Bacteria control 

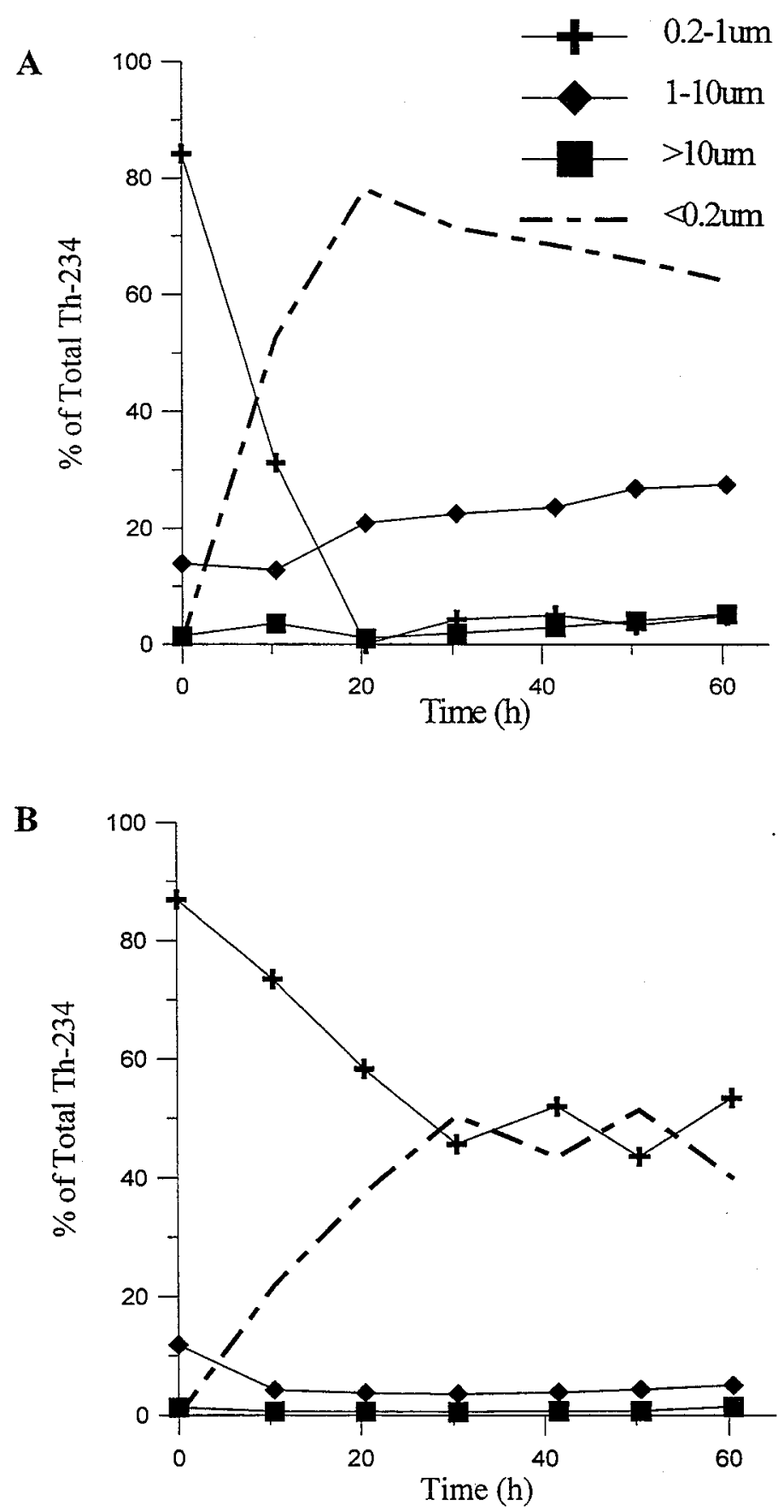

Figure 2.2 - Experimental series \#1, Uronema experiment. $\%$ of total Th-234 in various size fractions vs. time A: Grazing culture; B: Bacteria control 

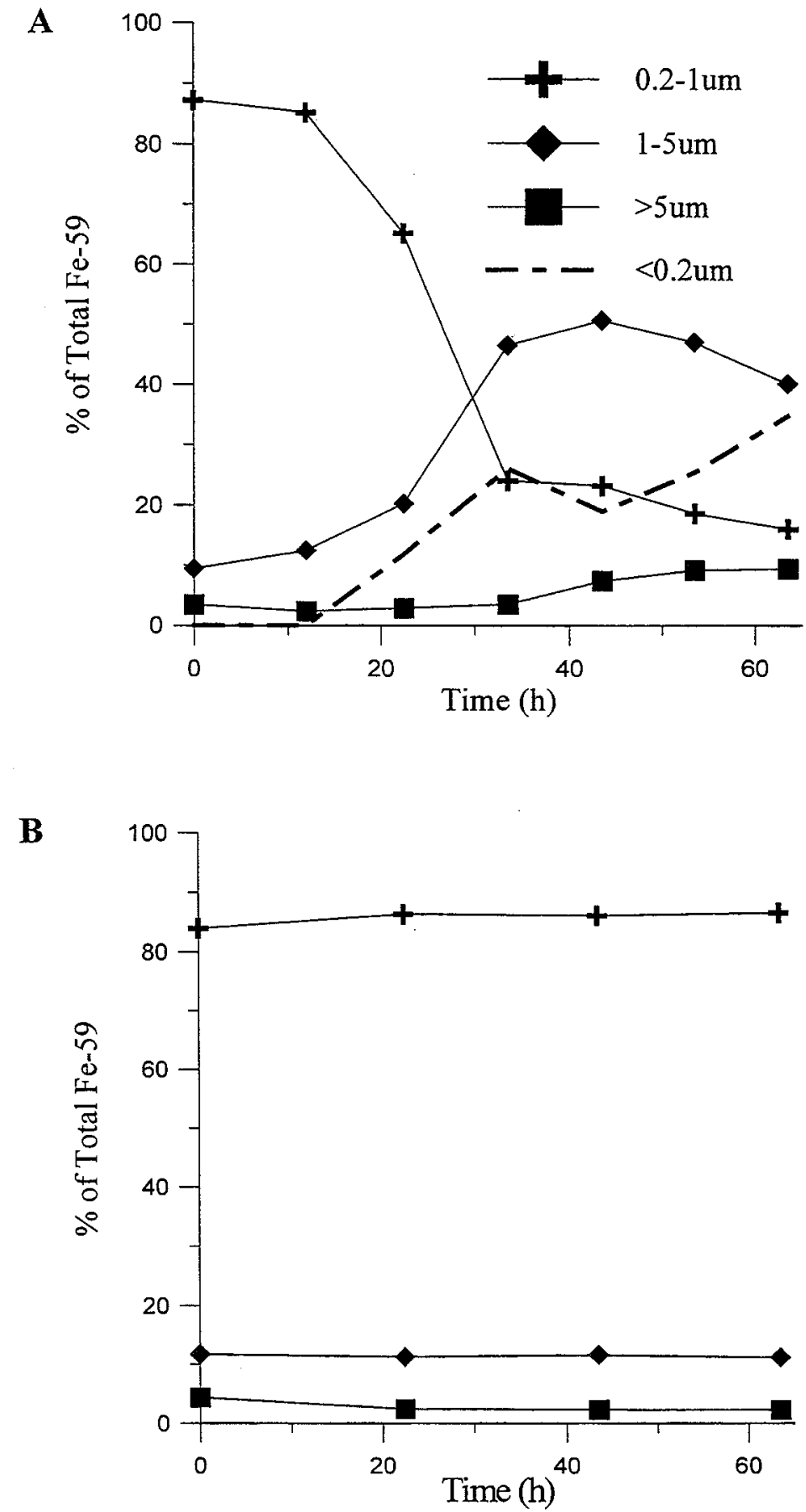

Figure 2.3 - Experimental series \#1, Paraphysomonas experiment. $\%$ of total $\mathrm{Fe}-59$ activity in various size fractions vs. time A: Grazing culture; B: Bacteria control 

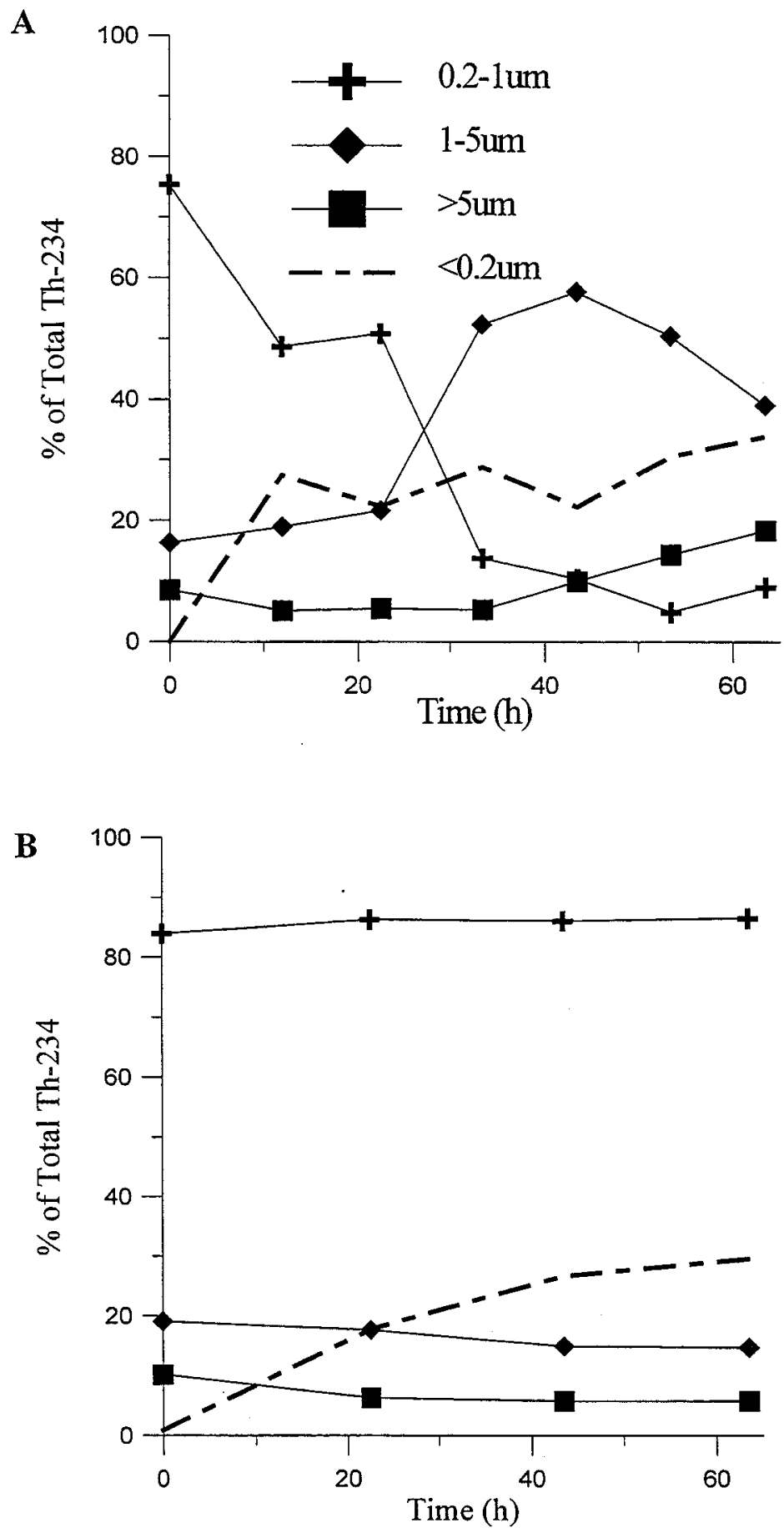

Figure 2.4 - Experimental series \#1, Paraphysomonas experiment. $\%$ of total Th-234 activity in various size fractions vs. time A: Grazing culture; B: Bacteria control 

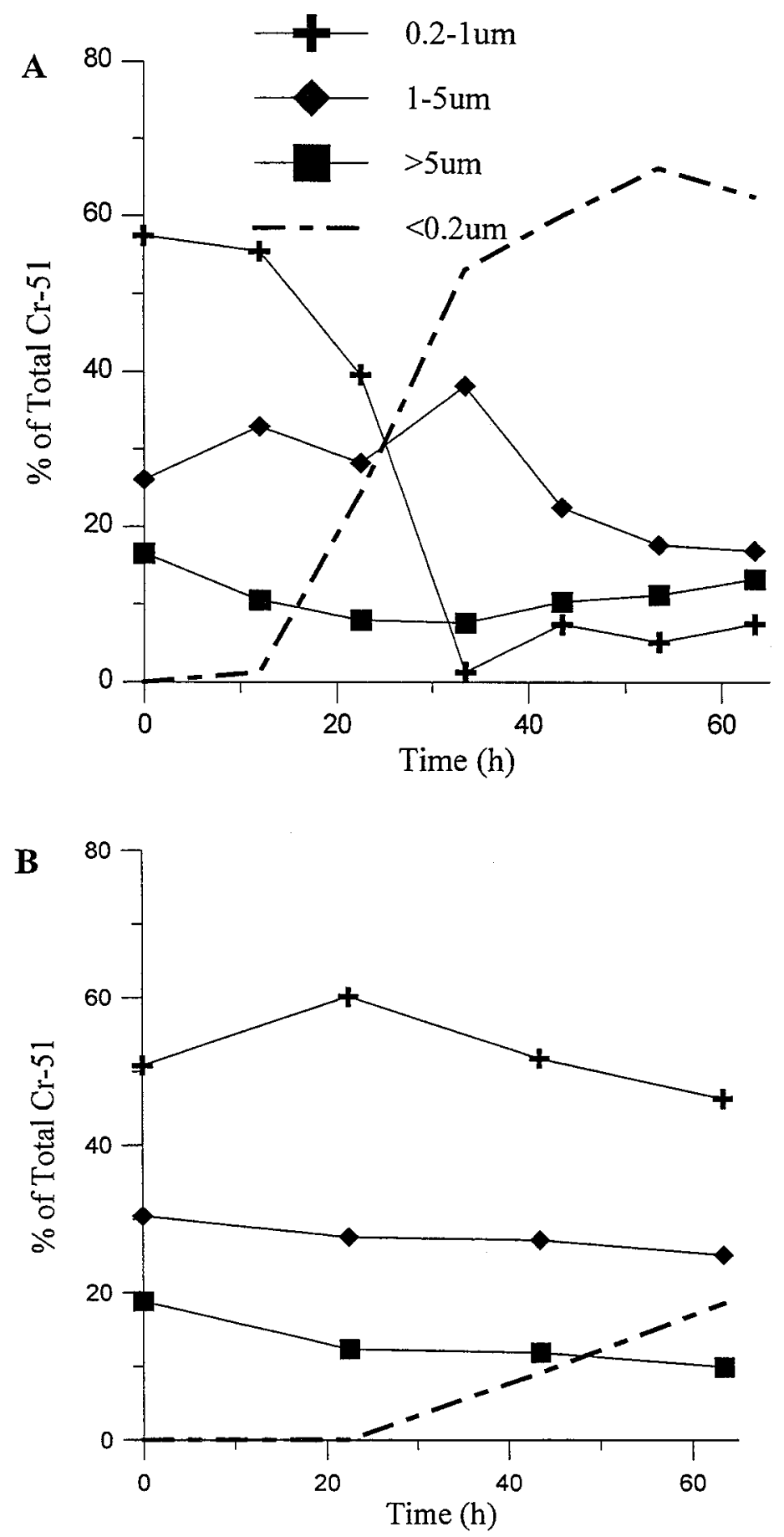

Figure 2.5 - Experimental series \#1, Paraphysomonas experiment $\%$ of total $\mathrm{Cr}-51$ activity in various size fractions vs. time A: Grazing cultures; B: Bacteria control 
bacterial size class. ${ }^{51} \mathrm{Cr}$ had a distribution in the control similar to that of ${ }^{59} \mathrm{Fe}$ and ${ }^{234} \mathrm{Th}$, except that there was more of it in the $1-5 \mu \mathrm{m}$ and $>5 \mu \mathrm{m}$ size classes. In the grazing cultures, as with Uronema, there was removal of all three radionuclides from the bacterial size class. Compared to the previous experiment with Uronema, there was a greater increase of ${ }^{59} \mathrm{Fe}$ and ${ }^{234} \mathrm{Th}$ in the $1-5 \mu \mathrm{m}$ size class and less release to the dissolved phase.

${ }^{51} \mathrm{Cr}$ in the grazing culture behaved very differently from the other nuclides, being moved from the particulate phase and into the $<0.2 \mu \mathrm{m}$ phase by the grazer to a much greater extent than ${ }^{59} \mathrm{Fe}$ and ${ }^{234} \mathrm{Th}$.

The last experiment in this series involved the grazer Cafeteria (Figs. 2.6-2.8). The behavior of the isotopes in the controls was similar to that observed in Figs. 2.1 and 2.2 from the Uronema experiment, with substantial desorption of ${ }^{234} \mathrm{Th}$ and, to a lesser extent, ${ }^{51} \mathrm{Cr}$. All of the isotopes in the experimental culture were removed from the bacterial size class and ended up almost completely in the dissolved phase. No movement of the isotopes into larger particle size classes was observed with this grazer.

\section{Discussion}

The preliminary experimental results shown here are easily subject to overinterpretation given the lack of experimental replicates, ancilliary data, and mechanistic information. Nonetheless, despite these limitations, these results demonstrated the potential for protozoan grazing to have significant and dynamic effects on the biogeochemical cycling of trace metals. For example, in many cases (especially the Cafeteria experiment) the action of the grazers resulted in an increase in the amount of the radiotracers in the $<0.2 \mu \mathrm{m}$ size class. This result implies either: 1) Remineralization of the tracers into the dissolved phase; or 2) Incorporation of the tracers into colloidal material less than $0.2 \mu \mathrm{m}$ in size. Based on what is known about protozoan digestion and ejecta, both scenarios are equally plausible, as is a mixture of the two. The stability of

${ }^{59} \mathrm{Fe}$ in the dissolved phase in some experiments, despite its known affinity for surfaces, seems to imply that Fe was stabilized in the dissolved phase by either ligand 

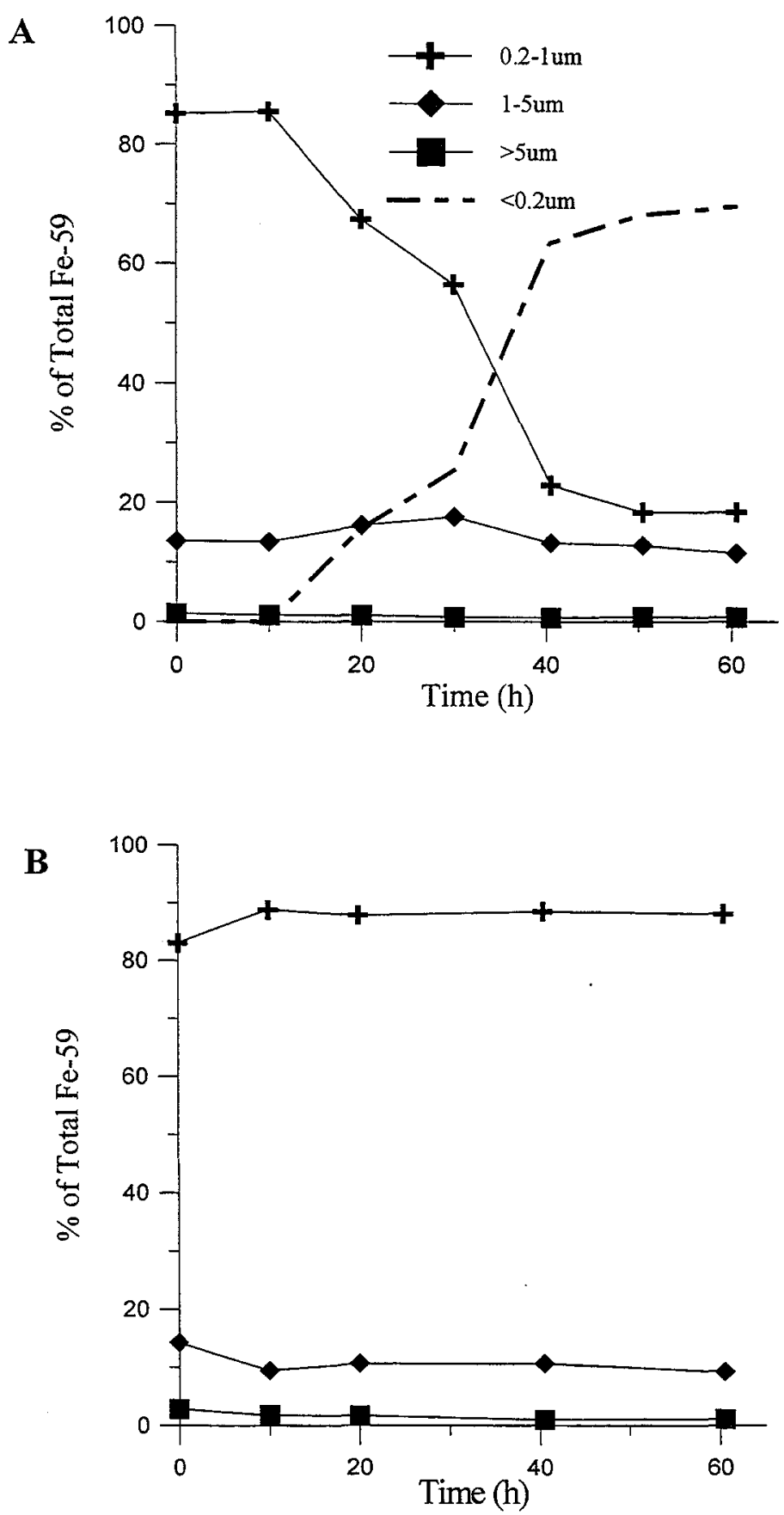

Figure 2.6 - Experimental series \#1, Cafeteria experiment $\%$ of total $\mathrm{Fe}-59$ activity in various size fractions vs. time A: Grazing culture; B: Bacteria control 

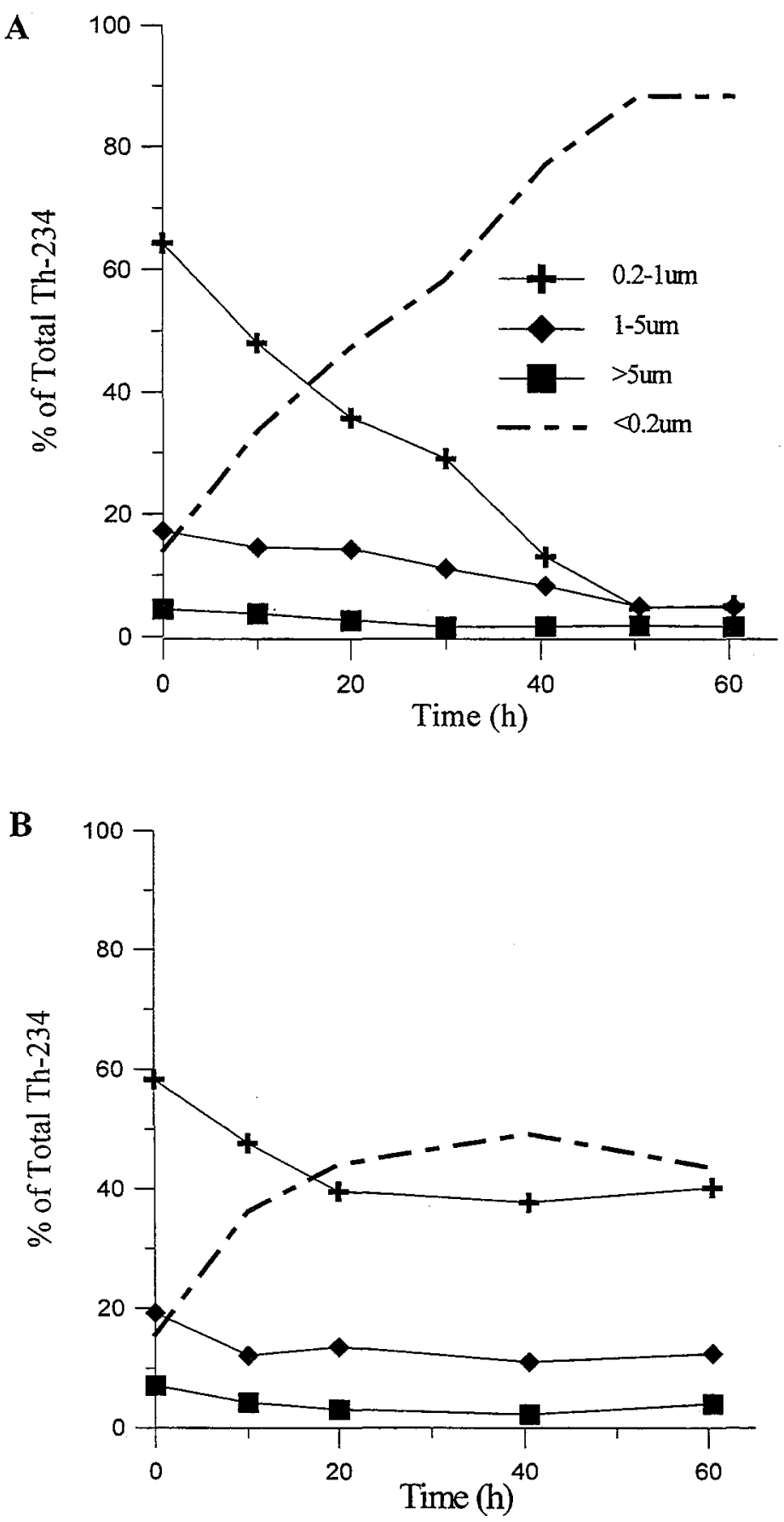

Figure 2.7 - Experimental series \#1, Cafeteria experiment. $\%$ of total Th-234 activity in various size fractions vs. time A: Grazing culture; B: Bacteria control 

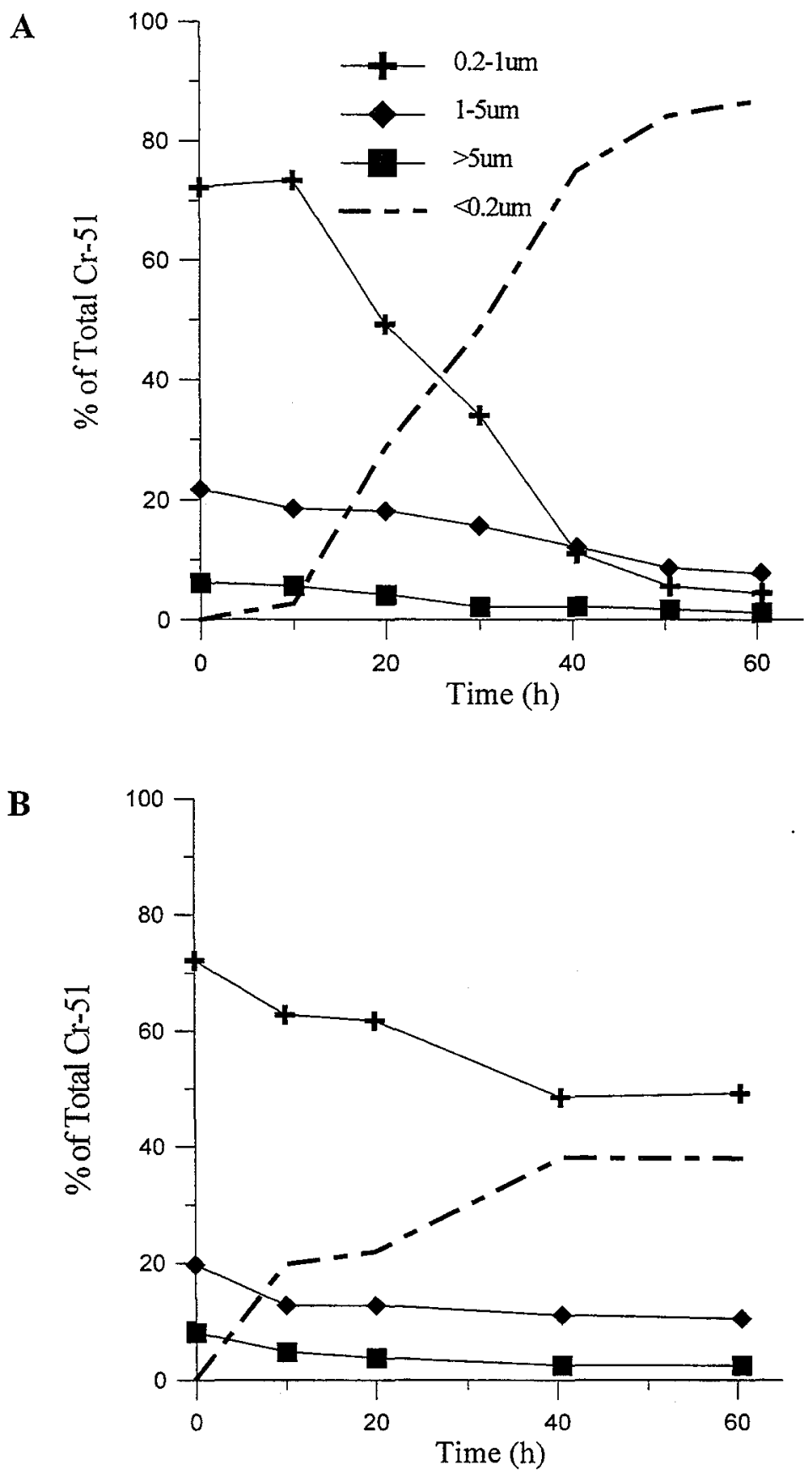

Figure 2.8 - Experimental series \#1, Cafeteria experiment. $\%$ of total $\mathrm{Cr}-51$ activity in various size fractions vs. time A: Grazing cultures; B: Bacteria control 
complexation or incorporation into small colloids. The same may be true of Th in these systems.

In several experiments, particularly those involving Paraphysomonas, grazing resulted in substantial changes in the particle size class distribution of the tracer, with an increase in the larger size fractions. In such cases, less of the tracers ended up in the $<0.2 \mu \mathrm{m}$ size class. This pattern could be due to several factors. One possibility is that the protozoa themselves were taking up the tracer. Another explanation is that the grazers produced waste particles in the $>1 \mu \mathrm{m}$ size class which either contained ejected tracer or adsorbed it from solution. Yet another alternative is that the action of the grazers produced aggregates which incorporated labeled small particles. This last possibility is supported by the observation that protozoan grazers tend to induce visible aggregation in culture, much more so than a pure bacterial culture.

In some cases, grazing may decouple the cycling of trace metals and organic carbon. While no measurements of dissolved or particulate organic carbon were made on these cultures, the conversion of bacterial biomass into protozoan biomass with average respiratory efficiency $(\sim 50 \%)$ should have resulted in a concurrent loss of particulate organic carbon. This suggests that, in cases where most of the tracer remained in the particulate phase (eg. ${ }^{234} \mathrm{Th}$ in the Paraphysomonas experiment), the action of the grazers may substantially increase metal/C ratios on particles, particularly in the 1-10 um size range, due to the preferential remineralization of carbon relative to metals.

Despite the similar size of the grazers used in these experiments, their effects on tracer distibutions showed some interesting variations. The primary effect of grazing by Cafeteria, for instance, appears to be an increase in the amount of tracer in the $<0.2 \mu \mathrm{m}$ phase. Paraphysomonas has the opposite effect, causing an increase of tracer in the larger particles. Uronema seems to fall somewhere in between the other two species, with both regeneration of the tracers into the dissolved phase and accumulation into larger particles observed. These differences may be related to grazer-specific characteristics, such as digestive metabolism. Additional factors could be differences in prey quality, or media composition. 
The difference in the behavior of $\mathrm{Cr}$ relative to $\mathrm{Fe}$ and Th in the Paraphysomonas experiment is intriguing. Cr may be stabilized in the dissolved phase more so than $\mathrm{Fe}$ or Th due to ligand complexation and the exceedingly slow reaction kinetics of $\mathrm{Cr}$ (III). Alternatively, $\mathrm{Cr}(\mathrm{III})$ could be oxidized to the relatively non-particle-reactive $\mathrm{Cr}(\mathrm{VI})$ within the protozoan food vacuole. A third possibility is that, if uptake onto $1-5 \mu \mathrm{m}$-sized particles in the Paraphysomonas experiment is due to assimilation of the tracers by the grazer itself, $\mathrm{Cr}$ (III) may be less available for uptake than $\mathrm{Fe}$ or $\mathrm{Th}$.

The preceding discussion is quite speculative, and this is in keeping with the preliminary nature of this first series of experiments. The results, while inconclusive, suggest an array of possibilities and provoke numerous questions. In experimental series \#2, an attempt was made to answer some of the questions and eliminate certain possibilities. In this second series of experiments a decision was made to focus on fewer species, in greater detail. Cafeteria and Paraphysomonas were chosen as representative of the two extremes of grazer-mediated transformations of particle-associated trace metals: Regeneration into the dissolved phase vs. aggregation into larger particle size classes. Experiments were performed in triplicate in order to ascertain the degree of variability in the biological processes. In addition to radiolabeled cultures, parallel nonlabeled cultures were run in order to sample for particulate and dissolved organic carbon as well as cell counts of bacteria and protists. More detailed size fractionation of radiolabeled cultures was performed, including ultrafiltration. These and other modifications to the general experimental protocol are described in more detail in the section that follows.

\section{Series \#2 - Subsequent Experiments}

\section{Method}

Set-up - This experimental series consists of two experiments, conducted one month apart. The first in the series, Part A, involved the grazer Cafeteria. The second in the series, Part B, involved the grazer Paraphysomonas. Because of the need to run bottles 
in triplicate, it was not logistically feasible to study both organisms simultaneously. To the extent that it was possible, identical conditions were maintained between the two experiments. This was of particular concern with regard to the bacterial prey, which had to be grown up and harvested separately for each experiment. In order to produce two successive cultures of bacterial prey as similar in quality as possible, an attempt was made to grow $H$. halodurans on glucose media rather than yeast extract; glucose being a simpler and more well-defined substrate than yeast extract.

Although $H$. halodurans did grow well on glucose, the seawater medium became quite acidic ( $\mathrm{pH} 4)$ due to the production of acidic metabolic by-products by the bacteria. It was felt that such low $\mathrm{pH}$ would inhibit uptake of the radiotracers by the bacterial cells, particularly surface-adsorbed trace metals like Th. An attempt was made to buffer the medium using various concentrations of borate, HEPES, and HEPPS. It was found that the buffers substantially slowed down bacterial growth, and were unable to keep medium $\mathrm{pH}$ above 6 at high cell densities. Therefore it was decided to grow the bacteria on $0.06 \%$ yeast extract, as previously, instead of on glucose. A stock solution of $10 \%$ yeast extract was mixed up freshly and used to enrich the bacterial medium for the first experiment. It was then frozen until needed for the next experiment approximately one month later.

Radiolabeled bacteria for experimental series \#2 were grown up using methods similar to the first experimental series. VSW was enriched with $0.06 \%$ yeast extract, the medium was inoculated with $H$. halodurans, spiked with ${ }^{59} \mathrm{Fe}$ and ${ }^{51} \mathrm{Cr}$ (about $30 \mu \mathrm{Ci} / \mathrm{l}$ each), neutralized for spike acidity, and put on a shaker. Parallel non-labeled bacterial cultures were set up at the same time. ${ }^{234} \mathrm{Th}$ was not added to the labeled cultures until they were 48 hours old, in order to minimize possible wall loss. ${ }^{234} \mathrm{Th}$ was extracted from 200g of uranyl nitrate for each of these experiments.) Both labeled and non-labeled cultures were incubated at room temperature on a shaker for 3 days. At the end of this time, they were in lag phase. The cultures were then put into the refrigerator overnight, harvested by centrifugation the next day, and then rinsed and resuspended 3x in VSW. The resulting bacterial concentrates were then stored overnight in the refrigerator. 
The following day, the bacterial concentrates were diluted in VSW to a final concentration of $2-3 \times 10^{7} \mathrm{cells} / \mathrm{ml}$. Six bottles of $1500 \mathrm{ml}$ radiolabeled bacterial suspension were set up in 2.5 liter polycarbonate bottles. Six more bottles of $2000 \mathrm{ml}$ non-labeled bacteria suspension were also set up. Half of the labeled and non-labeled bacteria suspensions were inoculated from an exponential growth phase culture of the protozoan grazer (Cafeteria in the first experiment, Paraphysomonas in the second) growing on rinsed, resuspended yeast-extract-grown bacteria. Protists were added to achieve an initial concentration of about $10^{4}$ cells $/ \mathrm{ml}$. Immediately after the inoculation, samples for $t_{0}$ were taken. Experimental and control bottles were incubated without shaking at $20-22^{\circ} \mathrm{C}$ under normal laboratory light for the duration of the experiment, 48 hours. Starting at $t_{0}$, sampling (as described below) was performed every 12 hours. Cultures were gently mixed by swirling or tipping bottles prior to taking all samples.

Sampling and Analysis - Radio-isotope samples - Size-fractionated samples $(0.2 \mu \mathrm{m}$, $1 \mu \mathrm{m}, 5 \mu \mathrm{m})$ were obtained by vacuum filtration at all time points, as described previously for experimental series \#1. Volumes filtered in these experiments were: $10 \mathrm{ml}$ for the $0.2 \mu \mathrm{m}$ size class; $25 \mathrm{ml}$ for the $1 \mu \mathrm{m}$ size class;and $50 \mathrm{ml}$ for the $5 \mu \mathrm{m}$ size class. In some cases, these volumes were reduced for samples taken during the later part of the grazing cultures due to clogging of the filters. At $\mathrm{t}_{0}$, additional $0.2 \mu \mathrm{m}$ filter samples of the control cultures were taken and rinsed with the Ti(III)/citrate-EDTA reagent of Hudson and Morel (1989) in order to remove loosely-bound extracellular ${ }^{234} \mathrm{Th},{ }^{51} \mathrm{Cr}$, and ${ }^{59} \mathrm{Fe}$. As in experimental series \#1, all filter fractions were normalized to a total sample taken at that time point in order to correct for wall loss.

In addition to the vacuum filtered samples, additional $0.2 \mu \mathrm{m}$-filtered samples were taken at $t_{0}, t_{24}$, and $t_{48}$ using a reverse-flow filtration technique driven by head pressure. This method of filtration is much slower and more gentle than vacuum filtration, and less likely to cause rupture of fragile protist cells and/or aggregation of particles at the filter surface (Nagata and Kirchman, 1990). It was used as a check for the accuracy of the $0.2 \mu \mathrm{m}$ vacuum-filtered samples. To make a reverse-flow filtration device, a $47 \mathrm{~mm}$ 
diameter $0.2 \mu \mathrm{m}$ pore size Nuclepore filter was glued with silicone adhesive around the edges to one end of an acid-washed piece of PVC pipe $47 \mathrm{~mm}$ in diameter and $8 \mathrm{~cm}$ in length. In order to use, the filter was pre-wet with VSW, and submerged to a depth of 1-2 inches in an aliquot of the experimental or control culture in a plastic cup. Head pressure at the surface of the filter caused water to pass through and slowly rise in the PVC pipe until the water level inside equaled the water level outside. Reverse-flow filtrate was withdrawn from within the PVC pipe using a plastic dispo-pipette. Reverse-flow filtrates $(10 \mathrm{ml})$ were placed in plastic cups and acidified with $\mathrm{HCl}$ for later radio-isotope counting. The activity in these filtrates was normalized to an equal volume of whole culture, also acidified and placed in plastic cups.

An additional $10 \mathrm{ml}$ aliquot of reverse-flow $0.2 \mu \mathrm{m}$ filtrate was used for ultrafiltration at $\mathrm{t}_{48}$. Ultrafiltration was performed using centrifugal concentrators with a nominal molecular weight cut-off of 10,000MWCO (Macroseps with Omega-type membrane, made by Filtron Technology Corporation, Northborough, MA). Prior to ultrafiltration, Macroseps were pre-treated with a dilute acid rinse $(10 \mathrm{ml}$ of $1 \mathrm{~N} \mathrm{HCl})$, followed by 2 rinses with Milli-Q water ( $10 \mathrm{ml}$ each) and one rinse with $10 \mathrm{ml}$ of $0.2 \mu \mathrm{m}$-filtered Sargasso seawater. After pre-treatment, Macroseps were stored in the refrigerator until needed for the experiment. Ultrafiltration was performed by loading the Macroseps with $10 \mathrm{ml}$ of reverse-flow filtrate and spinning on a centrifuge at $5000 \mathrm{x}$ g for 60 minutes. Volumes of filtrate and retentate were recorded. The entire retentate volume $(1.1 \mathrm{ml})$ was counted, and a $2 \mathrm{ml}$ aliquot of the filtrate $(8.9 \mathrm{ml}$ total) was counted. Radio-isotope counts for the ultrafiltration retentates and filtrates were normalized to the reverse-flow filtrate counts.

As in the first experimental series, all radio-isotope samples were counted on the lowenergy Canberra germanium gamma detector, to $5 \%$ error or less.

Organic Carbon Samples - Samples for organic carbon analysis were taken at all sampling times from the non-radioactively-labeled cultures. These samples were taken and analyzed by Elizabeth Kujawinski, who also developed the sample protocol. For 
particulate organic carbon (POC) samples, Nuclepore polycarbonate filters $(47 \mathrm{~mm}$ diameter, $0.2 \mu \mathrm{m}, 1 \mu \mathrm{m}$, and $5 \mu \mathrm{m}$ pore size) were loaded into acid-cleaned glass vacuum filter holders. Filters and filter holders were pre-rinsed with ethanol followed by Milli-Q water. A mixed aliquot of culture was then poured into the filter holder and gently filtered by vacuum as in the radio-isotope samples. Volumes were generally $40 \mathrm{ml}$ for the $0.2 \mu \mathrm{m}$ filter, $100 \mathrm{ml}$ for the $1 \mu \mathrm{m}$, and $200 \mathrm{ml}$ for the $5 \mu \mathrm{m}$. As for the radioisotope size factionation, these volumes were sometimes reduced if filters appeared to be clogging. Filtration was stopped just short of dryness, and the filter was transferred from the filtration apparatus to a $47 \mathrm{~mm}$ petri dish and immediately covered with $1 \mathrm{ml}$ of reagent alcohol.

Particulates were then gently and thoroughly scraped off the filter surface using a teflon-lined spatula. Particulates and reagent alcohol were quantitatively pipetted from the petri dish and transferred to a small Eppendorf tube. The filter was rinsed with $400 \mu \mathrm{l}$ reagent alcohol, also transferred to the Eppendorf tube. This procedure for cleaning particles off of the Nuclepore membranes was adapted from the protocol of Cowen (1991) for individual particle analysis by electron microscopy. Samples were stored under refrigeration until $\mathrm{CHN}$ analysis. Prior to $\mathrm{CHN}$ analysis, particulates in reagent alcohol were transferred to a pre-weighed Sn boat. Alcohol was slowly and completely evaporated on a hot plate set to low. POC samples were then analyzed on a Fisons Instruments EA 1108 Elemental Analyzer.

For dissolved organic carbon (DOC) samples, $40 \mathrm{ml}$ of culture was filtered by overpressure $\left(\mathrm{N}_{2}\right.$ gas, $\left.2-3 \mathrm{psi}\right)$ through a $0.2 \mu \mathrm{m}, 47 \mathrm{~mm}$ diameter Anopore membrane filter (Whatman) in an in-line teflon filter holder. The flow rate during this procedure was 2$3 \mathrm{ml} / \mathrm{minute}$. Filtrate was collected in a pre-combusted glass vial, acidified with $150-$ $200 \mu \mathrm{l}$ of $50 \% \mathrm{H}_{3} \mathrm{PO}_{4}$, capped, and stored in the refrigerator until analysis. Total organic carbon (TOC) samples ( $15 \mathrm{ml}$ whole culture aliquot $+15 \mathrm{ml}$ Milli- $\mathrm{Q}$ water) were similarly placed in glass vials, acidified, and stored under refrigeration. DOC and TOC samples were analyzed in the laboratory of Ed Peltzer at WHOI, using a homemade high- 
temperature combustion / discrete injection (HTC/DI) analyzer (Peltzer and Brewer, 1993), linked to a Li-Cor non-dispersive infra-red (NDIR) $\mathrm{CO}_{2}$ analyzer.

In addition to the DOC values measured directly from the Anopore filtrates as described above, an additional DOC value was calculated as the difference between the TOC value and POC collected on the $0.2 \mu \mathrm{m}$ Nuclepore filter. The difference between measured and calculated DOC values served as a check on the organic carbon mass balance obtained with these techniques. In the first experiment in series \#2, Part A (with Cafeteria), measured and calculated DOC values generally agreed to within $\sim 20 \%$, with no systematic offsets between the two. Mass balances improved somewhat in series \#2 Part B (with Paraphysomonas), with measured and calculated DOC values generally agreeing to within $\sim 12 \%$. Values for organic carbon $<0.2 \mu \mathrm{m}$ (DOC) shown in Fig. 2.10 are an average of the measured and calculated DOC values, with error bars representing the standard deviation between the two.

Additional samples - At each experimental sampling time, $\mathrm{pH}$ was determined on small aliquots $(10-20 \mathrm{ml})$ of the non-labeled control and experimental cultures, using an Orion Model 410A pH meter. Samples for cell counting by microscopy were also taken from non-labeled control and experimental cultures at each time point, and from the protistan inoculum culture at $\mathrm{t}_{0}$. Samples for microscopy $(9 \mathrm{ml}$ whole culture $+1 \mathrm{ml} 10 \%$ solution of glutaraldehyde in filtered VSW) were removed aseptically from cultures and placed in sterile $15 \mathrm{ml}$ centrifuge tubes. Samples were stored in the refrigerator for 1-2 weeks until slides were made. Slides were made by gentle vacuum filtration of sample onto $25 \mathrm{~mm}$ black $0.2 \mu \mathrm{m}, 1 \mu \mathrm{m}$, and $5 \mu \mathrm{m}$ Nuclepore filters and staining with acridine orange (AO) (Hobbie et. al., 1977). The Nuclepore filters were placed on top of larger pore-sized $25 \mathrm{~mm}$ Millipore filters in order to promote even dispersion of cells on the Nuclepore filter. $\mathrm{AO}$ was used at a final concentration of $0.01 \%$, for about $3 \mathrm{~min}$ per sample. After filtration, samples were rinsed once with sterile filtered Milli-Q water to remove excess stain. Filters were placed onto fogged glass slides, a drop of low-fluorescence immersion oil applied, and a coverslip. The coverslip was sealed around the edges with clear nail 
polish and the slides were stored in the freezer until counting. Samples were counted in Dave Caron's laboratory on a Zeiss standard microscope equipped for epifluorescence microscopy. Samples collected on $0.2 \mu \mathrm{m}$ filters were used for all bacteria and protist counts. Samples on $1 \mu \mathrm{m}$ and $5 \mu \mathrm{m}$ filters were used to visually determine the nature of the particles retained by those filters. The filter set used for fluorescence observations was a BP450-490 exciter filter, an FT510 chromatic beam splitter, and an LP520 barrier filter.

\section{$\underline{\text { Results }}$}

Figure 2.9 shows the changes in protist and bacteria cell concentrations in control and experimental bottles over the course of the experiments in series \#2. (Values shown are the average \pm standard deviation of three replicate bottles). While the bacteria cell concentrations in the controls remained relatively constant, the grazers in the experimental bottles reduced the bacterial population by about an order of magnitude. The Cafeteria cultures cleared more quickly than the Paraphysomonas cultures. It should be noted that the numbers reported for bacteria cells $/ \mathrm{ml}$ refer to counts of single bacteria cells. This number is accurate for the control cultures, but in the grazing cultures during the last 24 hours of the experiment, especially in Part B with Paraphysomonas as the grazer, there was a significant amount of bacterial biomass aggregated in clumps of cells, so that accurate cell number was difficult to determine by microscopical counts. Therefore the numbers shown for bacteria cell $\mathrm{s} / \mathrm{ml}$ in experimental cultures (just for $\mathrm{t}_{36}$ and $\mathrm{t}_{48}$ ) are an underestimate of the actual population. The protistan and bacterial cell concentrations in these model systems were 10-100 times those normally found in coastal environments (Valiela, 1995).

$\mathrm{pH}$ of the control and experimental cultures changed over the time course of both experiments (Table 2.1). Due to the respiratory activity of the grazers, the $\mathrm{pH}$ in the experimental cultures was consistently $0.3-0.4 \mathrm{pH}$ units lower than in the controls. There was a drop in $\mathrm{pH}$ of about 0.3 units in the control cultures over the course of 48 hours, reflecting the fact that the bacteria in the controls were metabolically active. The $\mathrm{pH}$ in both control and experimental bottles in Part B of series \#2 was significantly lower than 

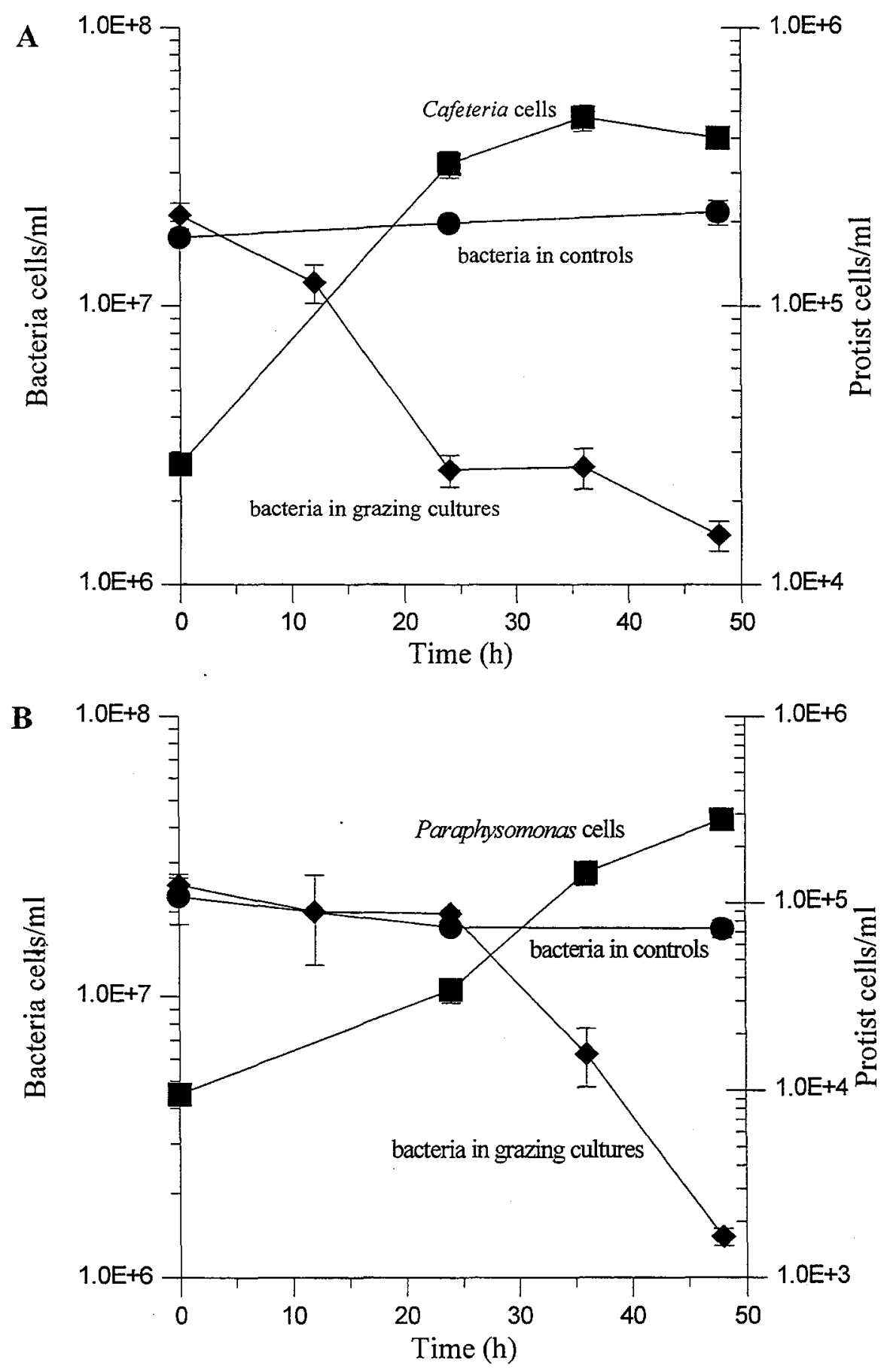

Figure 2.9 - Experimental series \#2 - Population dynamics Bacteria and protozoan cell concentrations vs time in grazing cultures and bacteria controls

A: Part A, Cafeteria as grazer

B: Part B, Paraphysomonas as grazer 
Table 2.1 - Experimental Series \#2, $\mathrm{pH}$ and total organic carbon (TOC) values for bacteria control and experimental (grazing) cultures from $t_{0}$ - $_{48}$. Part A experiment with Cafeteria as grazer; Part B - experiment with Paraphysomonas as grazer. All $\mathrm{pH}$ values are for individual bottles. TOC values at $\mathrm{t}_{0}, \mathrm{t}_{24}$, and $\mathrm{t}_{48}$ are the average and standard deviation of three replicate bottles. TOC values at $t_{12}$ and $t_{36}$ are for individual bottles.

\begin{tabular}{|c|c|c|c|c|}
\hline & $(\mathrm{Ca}$ & $\begin{array}{l}\text { rt A } \\
\text { eria } \text { expt.) }\end{array}$ & (Parapl & $\begin{array}{l}\text { rt B } \\
\text { somonas expt.) }\end{array}$ \\
\hline & pH & TOC $(\mathrm{mg} / \mathrm{l})$ & pH & TOC (mg/l) \\
\hline $\mathbf{t}_{0}$ control & 8.26 & $7.4 \pm 0.6$ & 7.83 & $19.4 \pm 1.5$ \\
\hline expt & 8.19 & $8.5 \pm 0.8$ & 7.76 & $20.0 \pm 1.3$ \\
\hline$t_{12}$ control & 8.23 & 8.0 & 7.57 & 17.9 \\
\hline expt & 8.06 & 7.4 & 7.52 & 16.4 \\
\hline$t_{24}$ control & 8.16 & $7.0 \pm 0.5$ & 7.34 & $14.5 \pm 0.8$ \\
\hline expt & 7.80 & $6.8 \pm 0.4$ & 7.25 & $14.5 \pm 1.5$ \\
\hline $\mathbf{t}_{36}$ control & 8.10 & 7.0 & 7.38 & 14.2 \\
\hline expt & 7.67 & 5.8 & 7.13 & 13.1 \\
\hline$t_{48}$ control & 7.99 & $7.1 \pm 0.1$ & 7.47 & $13.6 \pm 0.5$ \\
\hline expt & 7.76 & $5.3 \pm 0.5$ & 7.10 & $11.9 \pm 1.3$ \\
\hline
\end{tabular}


in Part A (eg. pH 7.8 vs 8.2 at $\mathrm{t}_{0}$ ). Values for total organic carbon (TOC) in experimental series \#2 are also shown in Table 2.1. In Part A, TOC levels in the control dropped slightly, from $7.4 \mathrm{mg} / \mathrm{l}$ at $\mathrm{t}_{0}$ to $7.1 \mathrm{mg} / \mathrm{l}$ at $\mathrm{t}_{48}$, reflecting metabolic activity of the bacteria and (most likely) uptake of DOC (see below). TOC levels in the experimental culture in Part A dropped further, down to $5.3 \mathrm{mg} / \mathrm{l}$ at $\mathrm{t}_{48}$, reflecting respiratory activity of the protozoans. TOC values in Part B were 2-2.5 times higher than in Part A, due to higher levels of DOC in that experiment (see below for more discussion). TOC levels in both control and experimental cultures in Part B started at 19-20 mg/1, and dropped to $12-$ $13 \mathrm{mg} / 1$ by $\mathrm{t}_{48}$.

The organic carbon in various particulate and dissolved size fractions changed over the course of the experiments, particularly in the grazing cultures (Fig. 2.10). (POC numbers at $t_{0}, t_{24}$, and $t_{48}$ are the average of three replicate bottles, shown with standard deviations. POC numbers at $t_{12}$ and $t_{36}$ are single values taken from one bottle; thus no error bar is shown. The same is true for the graphs of radionuclide size fractions that follow.) There was a decrease in organic carbon in the $0.2-1 \mu \mathrm{m}$ size class in the the grazing cultures, as expected due to removal of bacteria. This was accompanied by increases in organic carbon in larger size classes in the Paraphysomonas experiment, but not in the Cafeteria experiment.

Qualitative microscopic examination of AO-stained $0.2,1$, and $5 \mu \mathrm{m}$ filter samples in each experiment indicated that Cafeteria and Paraphysomonas cells were retained by the $1 \mu \mathrm{m}$ filter. The $5 \mu \mathrm{m}$ filters retained a few protist cells, but primarily bacterial aggregates. In the Cafeteria grazing cultures, the $5 \mu \mathrm{m}$ size fraction at $\mathrm{t}_{48}$ contained mostly filamentous and aggregated bacteria. In comparison, the $5 \mu \mathrm{m}$ filters in the Paraphysomonas experiment at $\mathrm{t}_{48}$ contained more extensive, amorphous bacterial aggregates, with no filamentous forms.

Relative to Part A (Cafeteria experiment), in Part B (Paraphysomonas experiment) there was about $10 \mathrm{mg} / \mathrm{l}$ more organic carbon in the dissolved phase at $t_{0}$ in both experimental and control cultures. The source of this DOC is not known. There was a draw-down of DOC in both control and experimental cultures in Part B, so that DOC 

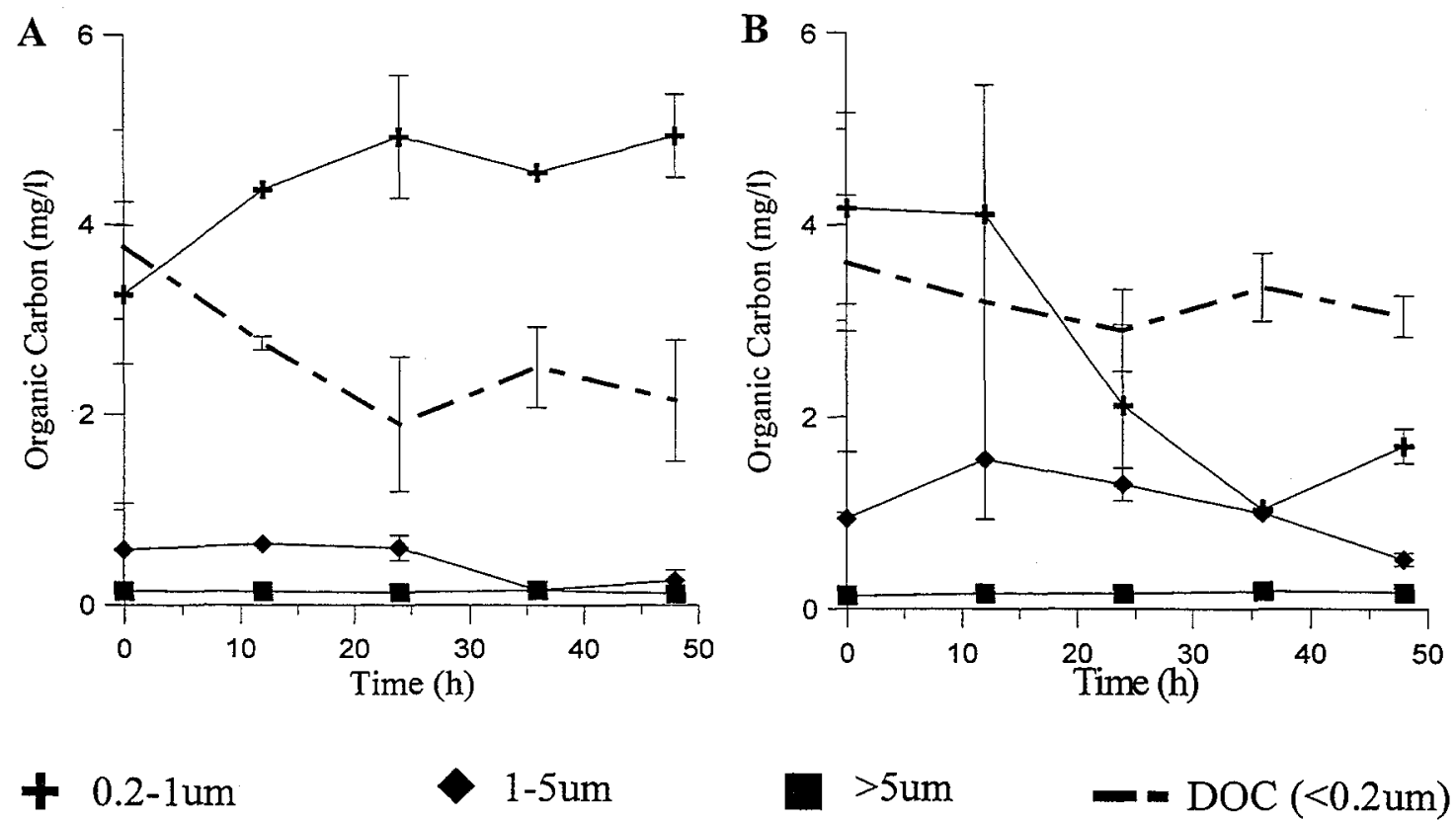

$\bullet 1-5 \mathrm{um}$

D >5um

- $\mathrm{DOC}(<0.2 \mathrm{um})$
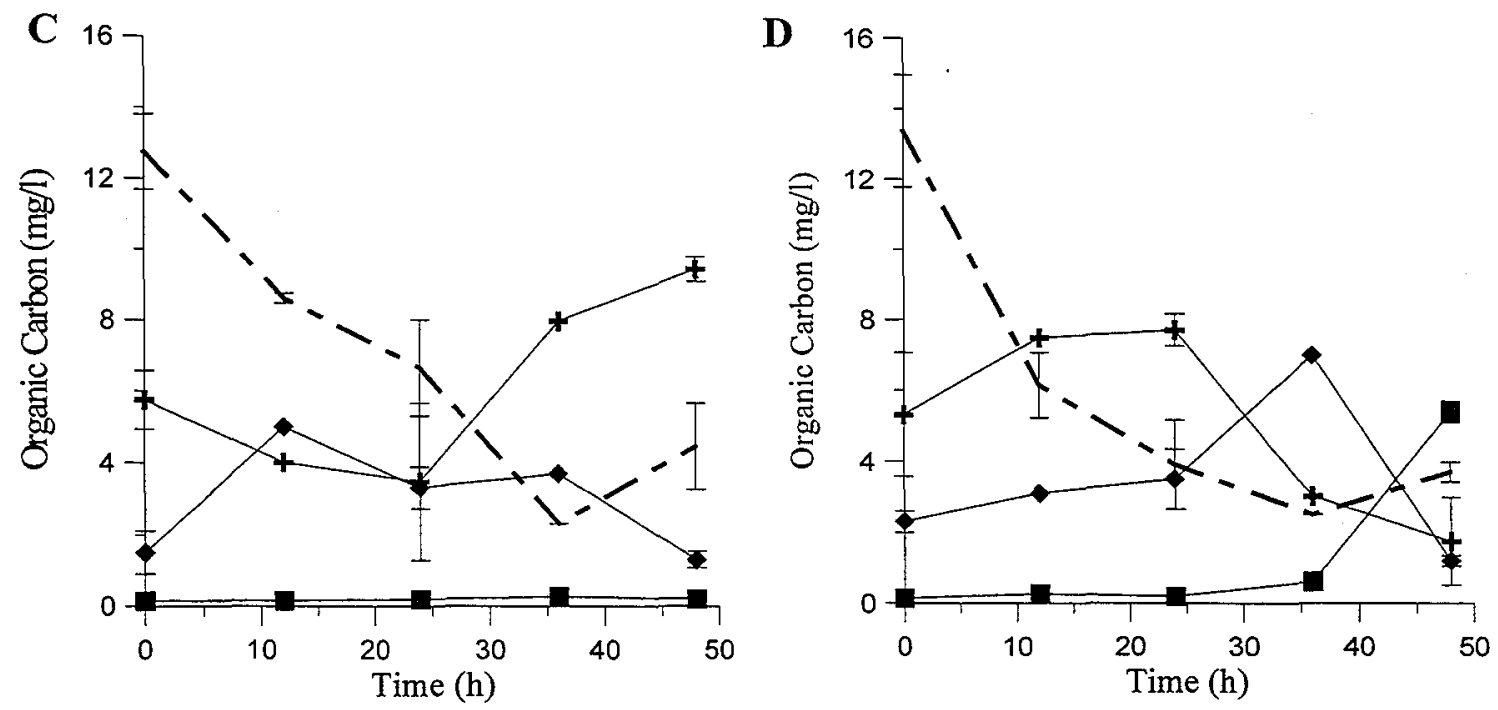

Figure 2.10 - Experimental series \#2 - Organic carbon size fractions vs time A: Cafeteria experiment (Part A), bacteria control B: Cafeteria experiment (Part A), grazing culture C: Paraphysomonas experiment (Part B), bacteria control D: Paraphysomonas experiment (Part B), grazing culture 
levels were similar to those in Part $\mathrm{A}$ by $\mathrm{t}_{36}$. There was also a draw-down of DOC in the control in Part A. The normal background level of DOC in VSW is about $1-1.5 \mathrm{mg} / \mathrm{l}$. DOC levels in the controls approached background levels by the end of the experiment, particularly in Part A, presumably due to uptake of DOC by the bacteria. Although DOC draw-down in both controls was accompanied by an increase in POC in the bacterial size class, this did not cause a change in bacterial cell numbers (see Fig. 2.9). The high DOC in the early stages of Part B may have been linked to the lower $\mathrm{pH}$ in those cultures, reflecting elevated metabolic activity due to DOC uptake by the bacteria in the controls and at the start of the grazing culture.

The Ti(III) rinse of the $>0.2 \mu \mathrm{m}$ size fraction of the control cultures at $\mathrm{t}_{0}$ in both experiments removed $80-85 \%$ of the ${ }^{234} \mathrm{Th}$, indicating that ${ }^{234} \mathrm{Th}$ was primarily adsorbed to the external surface of the bacteria cells (Table 2.2). The fact that the rinse removed comparatively little ${ }^{59} \mathrm{Fe}$ and ${ }^{51} \mathrm{Cr}$ (5-15\%) indicates that these nuclides were located intracellularly, or were bound very tightly to the cell exterior. $\mathrm{Cr}$ is more likely than $\mathrm{Fe}$ to be bound inertially to the cell exterior due to its slow exchange kinetics.

As in the case of organic carbon, there was removal of ${ }^{59} \mathrm{Fe}$ from the $0.2-1 \mu \mathrm{m}$ size class in both grazing cultures (Fig. 2.11). In the case of Cafeteria, this was accompanied by an increase of ${ }^{59} \mathrm{Fe}$ in the $<0.2 \mu \mathrm{m}$ size class and some increase in the amount of ${ }^{59} \mathrm{Fe}$ in the $>5 \mu \mathrm{m}$ size class. In the case of Paraphysomonas, there was some increase of ${ }^{59} \mathrm{Fe}$ in the $<0.2 \mu \mathrm{m}$ size class, but the largest effect was an increase in the $1-5 \mu \mathrm{m}$ and $>5 \mu \mathrm{m}$ size classes. The behavior of ${ }^{59} \mathrm{Fe}$ in the controls for both of these experiments (Part $\mathrm{A}$ and $\mathrm{B}$ ) was similar.

This was not the case for ${ }^{234} \mathrm{Th}$, shown in Figure 2.12. There was desorption of about $40 \%$ of the total ${ }^{234} \mathrm{Th}$ in the controls for the Cafeteria experiment (Part A), and little to ${ }_{\text {no }}{ }^{234} \mathrm{Th}$ desorption in the controls for the Paraphysomonas experiment (Part B). Since the concentration of bacteria and the amount of bacterial POC was similar in both of these experiments, the behavior of Th is indicative of significant differences in the surface chemistry of the bacterial prey in Parts A and B of experimental series \#2, despite attempts to keep conditions in both experiments as similar as possible. The behavior of 
Table 2.2 - Experimental Series \#2, Ti(III) wash results for bacteria controls at $\mathrm{t}_{0}$.

Values shown are the average and standard deviation of three replicates.

\begin{tabular}{|c|c|c|c|}
\hline & & ${ }^{1} \%>0.2 \mu \mathrm{m}$ & ${ }^{2} \%>0.2 \mu \mathrm{m}$ \\
\hline & & non-rinsed & Ti(III)-rinsed \\
\hline & ${ }^{334} \mathrm{Fe}$ & $100 \pm 0$ & $94.6 \pm 4.8$ \\
\hline Part A & ${ }^{51} \mathrm{Cr}$ & $\begin{array}{l}00.4 \pm 2.14 \\
100 \pm 0\end{array}$ & $91.7 \pm 8.5$ \\
\hline & ${ }^{59} \mathrm{Fe}$ & $100 \pm 0$ & $92.7 \pm 7.1$ \\
\hline Series \#2 & ${ }^{234} \mathrm{Th}$ & $100 \pm 0$ & $21.5 \pm 7.1$ \\
\hline Part B & ${ }^{51} \mathrm{Cr}$ & $100 \pm 0$ & $83.8 \pm 5.0$ \\
\hline
\end{tabular}

1 - Percentage of total activity retained by a $>0.2 \mu \mathrm{m}$ filter without rinsing.

2- Percentage of total activity retained by a $>0.2 \mu \mathrm{m}$ filter rinsed with Ti(III) reagent. 

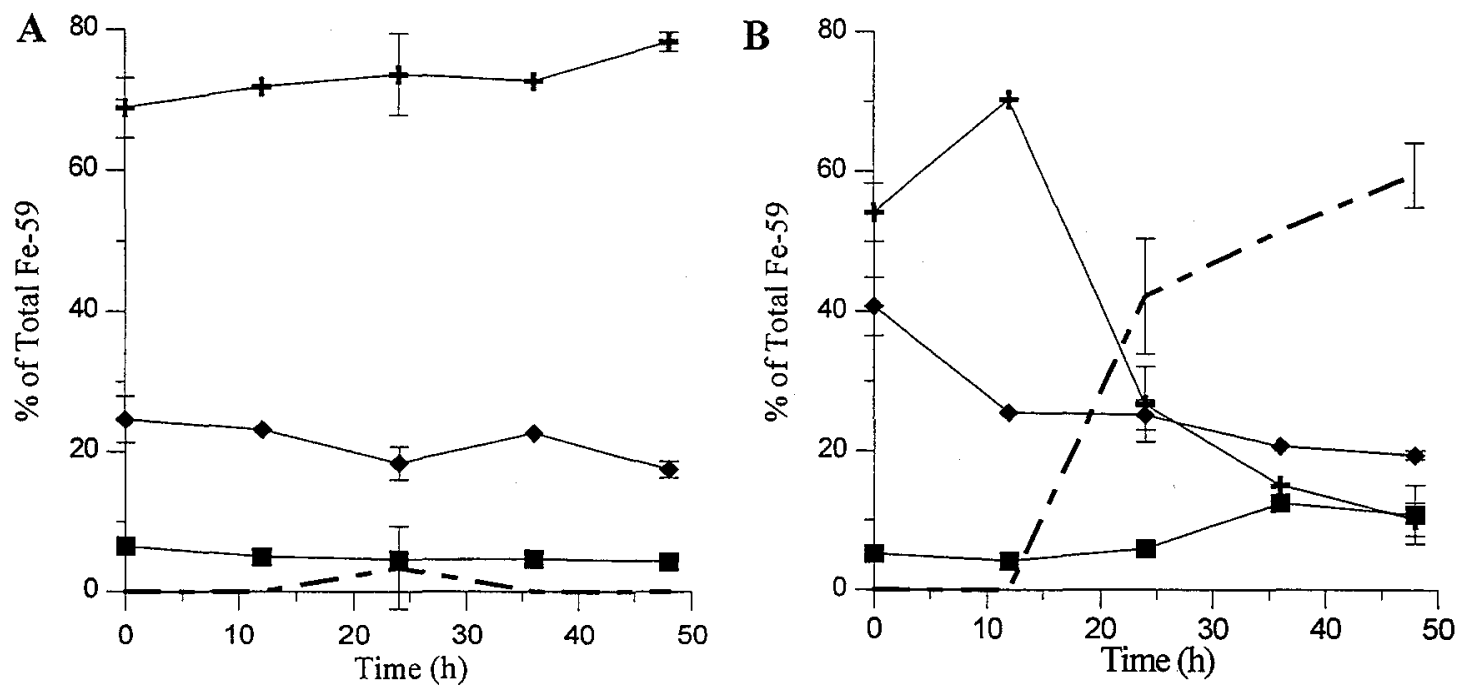

$+0.2-1$ um
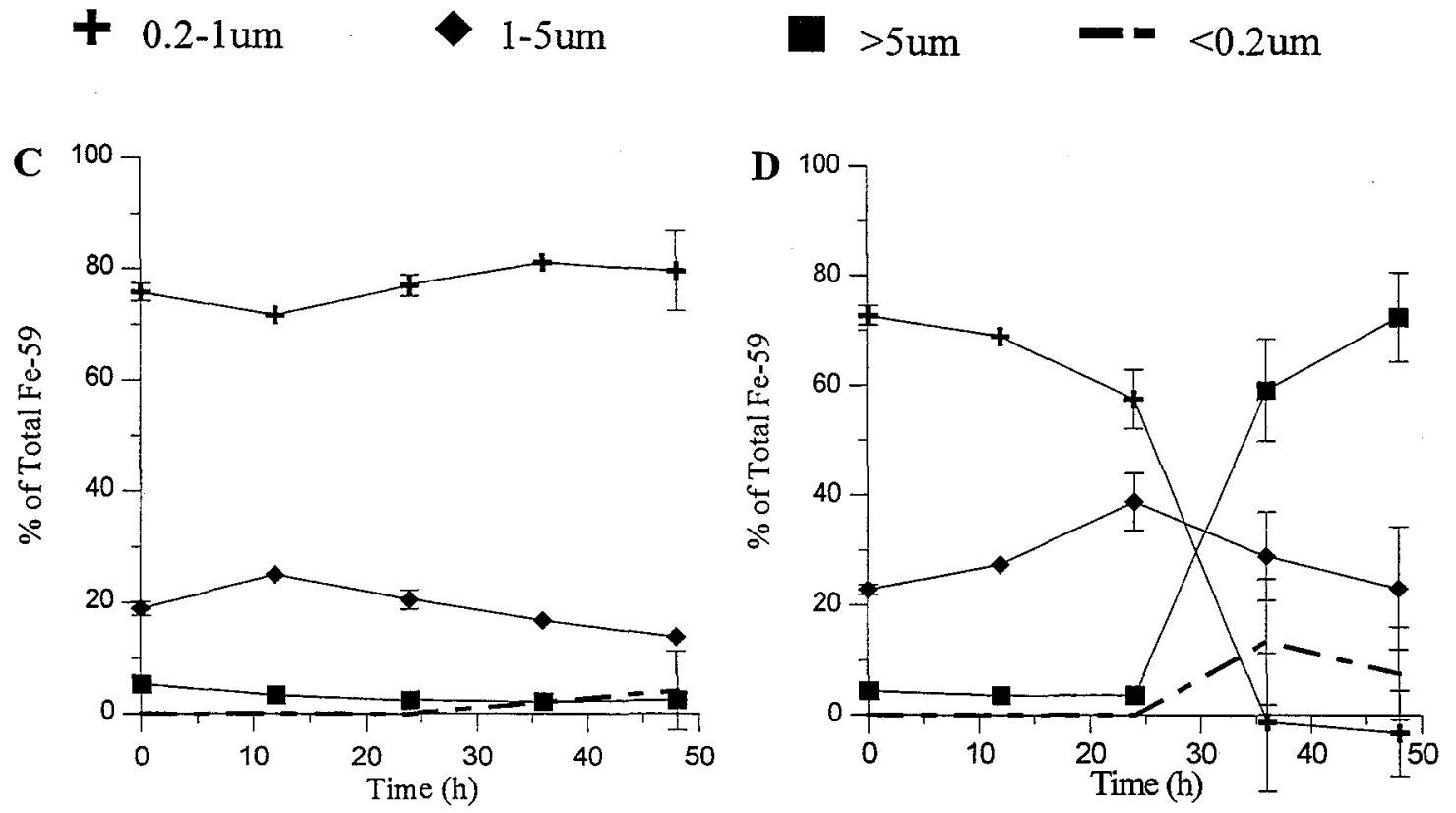

Figure 2.11 - Experimental series \#2 - Fe-59 size fractions vs time A: Cafeteria experiment (Part A), bacteria control B: Cafeteria experiment (Part A), grazing culture C: Paraphysomonas experiment (Part B), bacteria control D: Paraphysomonas experiment (Part B), grazing culture 

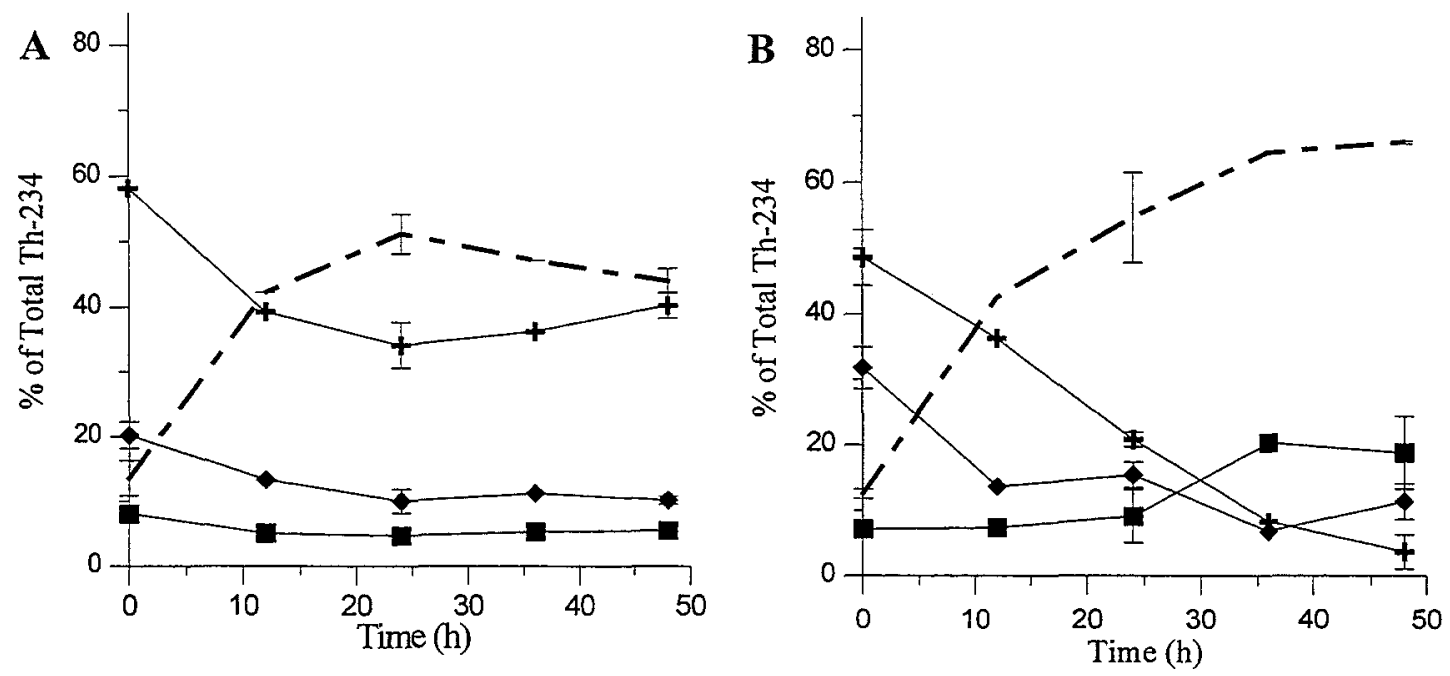

$+0.2-1$ um

$1-5 u m$

口 $>5 \mathrm{um}$

$-\mathbf{m}<0.2 \mathrm{um}$
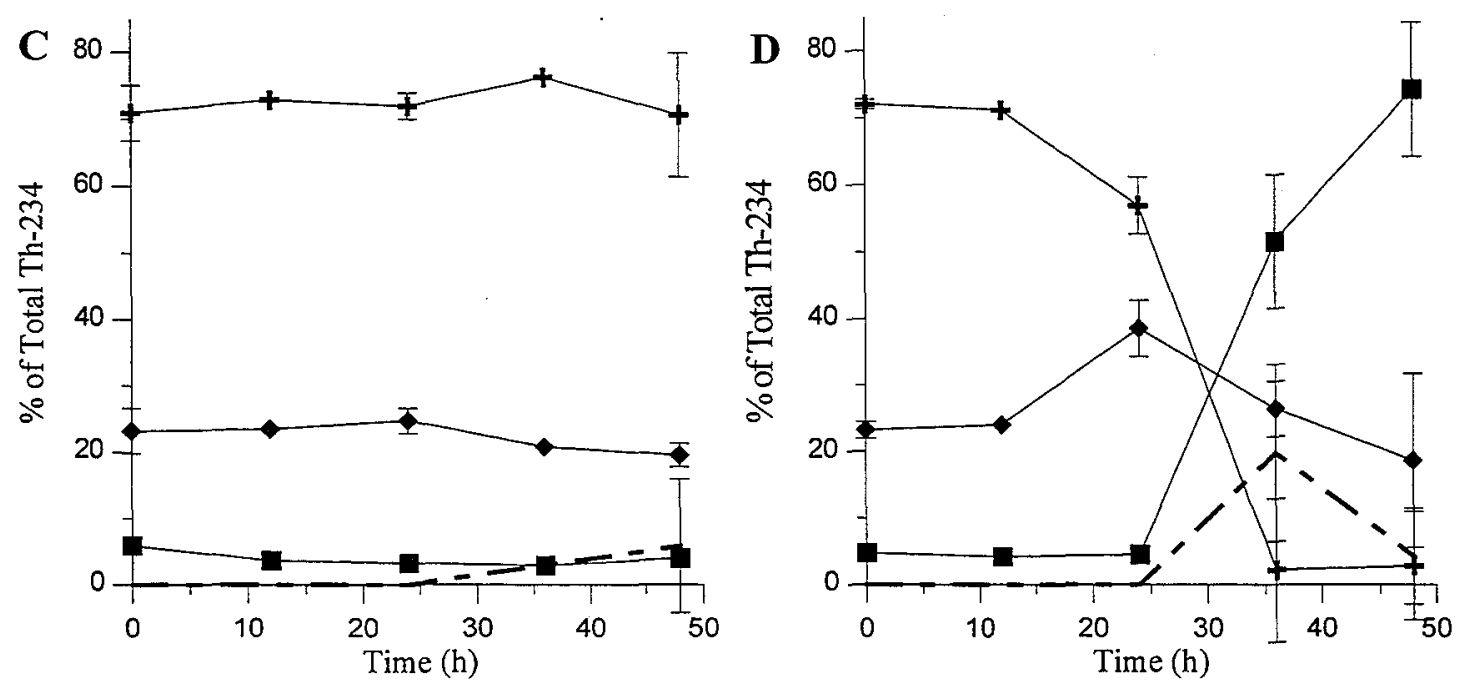

Figure 2.12 - Experimental series \#2 - Th-234 size fractions vs time

A: Cafeteria experiment (Part A), bacteria control

B: Cafeteria experiment (Part A), grazing culture

C: Paraphysomonas experiment (Part B), bacteria control

D: Paraphysomonas experiment (Part B), grazing culture 
${ }^{234}$ Th in the controls in Part B may have been linked to the presence of higher DOC in those cultures relative to the controls in Part A. The behavior of ${ }^{234} \mathrm{Th}$ in the grazing cultures of both Cafeteria and Paraphysomonas was very similar to that of ${ }^{59} \mathrm{Fe}$, as shown in Fig. 2.11.

Although the behavior of ${ }^{51} \mathrm{Cr}$ in experimental series \#2 was similar in many respects to that of ${ }^{59} \mathrm{Fe}$ and ${ }^{234} \mathrm{Th}$, two important differences in the grazing cultures should be noted (Fig. 2.13). In the grazing culture of Part A with Cafeteria, there was no increase in ${ }^{51} \mathrm{Cr}$ in the $>5 \mu \mathrm{m}$ size class as there was for ${ }^{59} \mathrm{Fe}$ and ${ }^{234} \mathrm{Th}$. In Part B with Paraphysomonas, the increase in ${ }^{51} \mathrm{Cr}$ in the $<0.2 \mu \mathrm{m}$ size class was as great as the increase in ${ }^{51} \mathrm{Cr}$ in the $>5 \mu \mathrm{m}$ size class. This is in sharp contrast to the behavior of ${ }^{234} \mathrm{Th}$ and ${ }^{59} \mathrm{Fe}$, which both tended to go mostly into the $>5 \mu \mathrm{m}$ size class in the Paraphysomonas grazing cultures. These results are consistent with the data from experimental series \#1 (see Figs. 2.3-2.5), where ${ }^{51} \mathrm{Cr}$ in the Paraphysomonas experiment also showed a much greater tendency to go into the $<0.2 \mu \mathrm{m}$ size class than did ${ }^{59} \mathrm{Fe}$ and ${ }^{234} \mathrm{Th}$.

The results of reverse-flow filtration and ultrafiltration in the grazing cultures of experimental series \#2 at $\mathrm{t}_{48}$ are shown in Table 2.3. The values obtained from reverseflow filtration agree within error $( \pm 5 \%)$ with the values obtained from vacuum filtration, with the exception of ${ }^{234} \mathrm{Th}$ and ${ }^{59} \mathrm{Fe}$ in Part B. In Part B, the amount of ${ }^{234} \mathrm{Th}$ and ${ }^{59} \mathrm{Fe}$ in the $<0.2 \mu \mathrm{m}$ size class obtained via vacuum filtration varied by over $100 \%$ and, for ${ }^{234} \mathrm{Th}$, was $20-60 \%$ less than the $<0.2 \mu \mathrm{m}$ size fraction obtained via reverse-flow filtration. Ultrafiltration results indicate that there was $2-2.5$ times more ${ }^{59} \mathrm{Fe}$ and ${ }^{234} \mathrm{Th}$ in the colloidal size class (i.e. \% retentate, $<0.2 \mu \mathrm{m}$ and $>10,000 \mathrm{MWCO}$ ) in Part B than in Part A. The $70 \%$ recoveries for ${ }^{59} \mathrm{Fe}$ in both Parts $\mathrm{A}$ and $\mathrm{B}$; and $85 \%$ recovery for ${ }^{234} \mathrm{Th}$ in Part $B$ may have been due to the retention within the ultrafilter of colloidal phases of these metals just under $10,000 \mathrm{MWCO}$ in size. Alternatively, the filter itself could have scavenged dissolved forms of the metals. In contrast to ${ }^{234} \mathrm{Th}$ and ${ }^{59} \mathrm{Fe},{ }^{51} \mathrm{Cr}$ demonstrated only about $10-15 \%$ partitioning into the colloidal size class in both Parts $\mathrm{A}$ and $\mathrm{B}$, and no loss during ultrafiltration. 

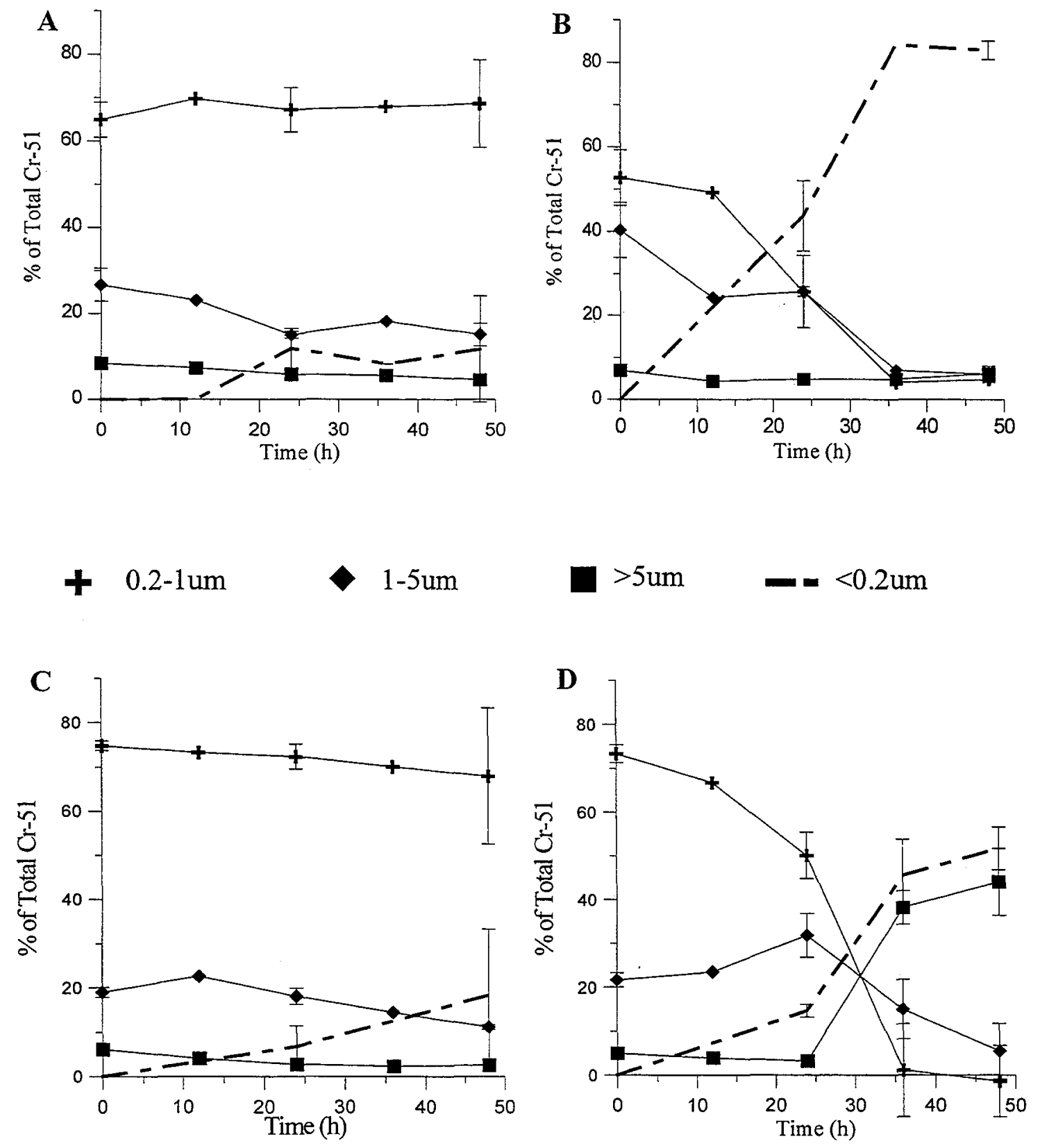

Figure 2.13 - Experimental series \#2 - Cr-51 size fractions vs time

A: Cafeteria experiment (Part A), bacteria control

B: Cafeteria experiment (Part A), grazing culture

C: Paraphysomonas experiment (Part B), bacteria control

D: Paraphysomonas experiment (Part B), grazing culture 
Table 2.3 - Experimental Series \#2 - vacuum filtration, reverse-flow filtration, and ultrafiltration results in experimental (grazing) cultures at $t_{48}$. Values shown are the average and standard deviation of three replicates.

\begin{tabular}{|c|c|c|c|c|c|}
\hline & \multirow{2}{*}{$\begin{array}{c}\%<0.2 \mu \mathrm{m} \\
\text { vacuum } \\
\text { filtration }\end{array}$} & \multirow{2}{*}{$\begin{array}{l}2 \%<0.2 \mu \mathrm{m} \\
\text { reverse-flow } \\
\text { filtration }\end{array}$} & \multicolumn{3}{|c|}{ - } \\
\hline & & & ${ }^{{ }_{\%} \% \text { Retentate }}$ & ${ }^{4} \%$ Filtrate & \% Recovery \\
\hline${ }^{59} \mathrm{Fe}$ & $59.5 \pm 4.6$ & $53.3 \pm 0.9$ & $21.9+2.2$ & $45.8+2.1$ & $67.7 \pm 4.3$ \\
\hline rt A ${ }^{234} \mathrm{Th}$ & $66.1 \pm 0.2$ & $69.5 \pm 3.3$ & $22.2 \pm 0.6$ & $72.9 \pm 4.1$ & $95.1 \pm 4.7$ \\
\hline & $83.1 \pm 2.2$ & $86.2 \pm 7.7$ & $12.1 \pm$ & $90.2 \pm 3.2$ & $102.3 \pm 4.1$ \\
\hline $\mathrm{Fe}$ & $7.6 \pm 8.5$ & $8.1 \pm 0.2$ & $52.9 \pm 7.4$ & $14.6 \pm 8.0$ & $67.5 \pm 15.4$ \\
\hline rt B B ${ }^{234} \mathrm{Th}$ & $4.2 \pm$ & $18.2 \pm 0.1$ & & $44.9 \pm$ & $84.8 \pm 9.3$ \\
\hline & $51.8 \pm 4.9$ & $56.6 \pm 1.5$ & $15.3 \pm 1.4$ & $86.0 \pm 1.7$ & $101.3 \pm 3.1$ \\
\hline
\end{tabular}

$1-\%$ of total activity passing through $0.2 \mu \mathrm{m}$ cutoff, using vacuum filtration

2 - $\%$ of total activity passing through $0.2 \mu \mathrm{m}$ cutoff, using reverse-flow filtration

3 - Retentate is $>10,000 \mathrm{MWCO},<0.2 \mu \mathrm{m}$.

4 - Filtrate is $<10,000 \mathrm{MWCO}$ 


\section{$\underline{\text { Discussion }}$}

As originally intended, experimental series \#2 was to have been a comparison between two different protozoan grazers (Cafeteria and Paraphysomonas) and their effects on trace metal chemistry. It is readily apparent, however, that Parts A and B of experimental series \#2 differed in more respects than just the species of protozoan involved. The high levels of DOC at $t_{0}$ in Part B relative to Part A are one indication that the chemistry of the two systems may have been different. This idea is reinforced by the behavior of ${ }^{234} \mathrm{Th}$ in the controls: extensive desorption in Part A; no desorption in Part B. The behavior of ${ }^{234} \mathrm{Th}$ in Part B could have been related to the higher levels of DOC in that experiment. One possibility is that, in taking up the (approximately) $13 \mathrm{mg} / 1 \mathrm{DOC}$ present in Part B (vs. 3-4 mg/l DOC in Part A), the bacteria may then have become limited by some other nutrient, such as phosphorus. Under such conditions, bacteria do tend to become more "sticky" (D. Caron, personal communication); this stickiness may possibly have accounted for the lack of Th desorption in Part B. This interpretation, however, is at present only speculation.

It is difficult to say how the higher concentrations of DOC in Part B may have influenced the system and contributed to differences in the experimental results in Parts $A$ and B. The source of this DOC is unclear - it could have derived from bacterial lysis or exudation during processing prior to setting up the experiment, or it could have derived from a contamination in the VSW used in Part B. In any case, based on the data for organic carbon and ${ }^{234} \mathrm{Th}$, it is possible that the chemistry of the model systems in Parts $\mathrm{A}$ and $\mathrm{B}$ of experimental series \#2 differed substantially. This must be considered in data interpretation, and a mechanistic comparison of the results from Parts $A$ and $B$ based largely on interspecies differences is not possible. Factors such as DOC concentration and prey surface chemistry must also be considered.

Intracellular vs extracellular trace metals - As shown from the Ti(III) rinse data in Table $2.2,{ }^{59} \mathrm{Fe}$ and ${ }^{51} \mathrm{Cr}$ were located primarily intracellularly, while ${ }^{234} \mathrm{Th}$ appeared to be extracellular. In all of the experiments discussed in this chapter, including both 
experimental series $\# 1$ and $\# 2$, the fate of $\mathrm{Fe}$ and $\mathrm{Th}$ was very similar. This indicates that the location of the metal either intracellularly or extracellularly on the prey makes very little difference to its eventual fate as a result of protozoan grazing. This is somewhat contradictory to the results of Reinfelder and Fisher, 1991. In studies of marine copepods feeding on diatoms radiolabeled with a variety of trace metal radio-isotopes, these workers found a very strong correlation between the copepod assimilation efficiency for a given trace element and the fraction of the element contained in the cytoplasm of the diatoms. This led Reinfelder and Fisher to hypothesize that copepods absorb only the soluble (i.e. cytoplasmic) portions of their algal prey.

Admittedly, in the studies described in this chapter, the assimilation efficiencies of the protists for $\mathrm{Fe}$ and $\mathrm{Th}$ were not measured. There should, however, be a correlation between the degree of assimilation of an element by the grazer and its eventual fate. If the degree to which $\mathrm{Th}$ and $\mathrm{Fe}$ were solubilized by the grazers in these experiments differed as a result of Th being located extracellularly and Fe being located intracellularly, then one should expect to see differences in the redistribution of these elements among various particulate and dissolved size classes as a result of protozoan grazing. In no case were substantial differences observed. This indicates that protists were able to solubilize extracellular $\mathrm{Th}$ to the same degree as intracellular Fe.

Given what is known about the digestive processes of protists, this makes sense. Protozoan grazers are single-celled phagotrophic organisms, and their digestion is of necessity intracellular. In intracellular digestion, the initial $\mathrm{pH}$ can become quite acidic (around 2 (Fok et. al., 1982)), and during subsequent steps hydrolytic enzymes operative at pH 5 essentially dissolve bacterial membrane components (Fok et. al., 1984). Such processes might reasonably be expected to solubilize $\mathrm{Th}$, bound non-specifically to external membranes. Organisms like copepods, which practice extracellular digestion, utilize a combination of mechanical disruption and digestive enzymes at circumneutral $\mathrm{pH}$ to obtain soluble nutrients from their prey (Vonk, 1960). Differences in the chemistry of intracellular vs. extracellular digestion may acount for the larger grazer-mediated 
fractionation between membrane-bound and cytoplasmic trace metals observed by Reinfelder and Fisher (1991), compared to this study.

It should be noted that the experiments conducted in this work were not designed to cause iron limitation of the protozoan grazers. Under such conditions, if $\mathrm{Fe}$ as a necessary nutrient was in short supply, one might expect $\mathrm{Fe}$ to be assimilated more efficiently than non-nutrient Th by protists, thereby resulting in a different repartitioning of the two metals as a result of grazing.

Organic complexation of trace metals - The most outstanding feature of the data for the Cafeteria grazing culture in Part A of experimental series \#2 is that all of the trace metal isotopes, ${ }^{59} \mathrm{Fe},{ }^{234} \mathrm{Th}$, and ${ }^{51} \mathrm{Cr}$, were overwhelmingly regenerated to the $<0.2 \mu \mathrm{m}$ size class. Ultrafiltration results in Table 2.3 indicate that a major fraction of these metals $<0.2 \mu \mathrm{m}$ were in the truly dissolved phase, as defined by $10,000 \mathrm{MWCO}$ (ultrafiltrate $50 \%$ for ${ }^{59} \mathrm{Fe}, 70 \%$ for ${ }^{234} \mathrm{Th}, 90 \%$ for ${ }^{51} \mathrm{Cr}$ ). The stability of particle reactive $\mathrm{Fe}$ and $\mathrm{Th}$ in the dissolved phase is striking, and contrary to expectation in a culture system where high particle concentrations provide abundant surface area for the adsorption of such trace metals. One explanation for this seeming paradox is that both $\mathrm{Fe}$ and $\mathrm{Th}$ were stabilized in the dissolved phase due to complexation by organic ligands.

For Th, organic complexation is rarely considered as an influence on dissolved/solid partitioning. If adsorption of $\mathrm{Th}$ to bacterial surfaces and subsequent ingestion by protozoan grazers can result in the eventual regeneration of $\mathrm{Th}$ to the dissolved phase in the form of organic complexes, this could reduce the overall scavenging of Th by particles. Coale and Bruland (1987) postulated that ${ }^{234} \mathrm{Th}$ could be stabilized against scavenging in the oceanic surface stratified euphotic zone as a result of the rapid particle cycling of the microbial loop. Our results suggest that the organic complexation of Th as a result of grazing in the microbial loop could contribute to the stability of Th in the stratified zone, and possibly at other times and in other locations.

$\mathrm{Fe}$, thought to be a limiting nutrient in some areas of the oceans, has recently been shown to be almost entirely complexed in the dissolved phase by organic ligands (Rue 
and Bruland, 1995; Wu and Luther 1995). Siderophores secreted by marine bacteria are often postulated as a potential source of the strong iron binding ligands recently found in seawater. Our data suggest that protozoan grazing may be an additional source of iron binding ligands in seawater, through the regeneration of iron from bacterial biomass. The strength of the iron binding ligands produced in our model systems (if any) is unknown.

The strength of any iron binding ligands regenerated into the dissolved phase as a result of protozoan grazing may vary with the degree of iron-limitation in the system. Chase and Price (1998), who studied the iron limitation of protozoan grazers (Paraphysomonas sp.), found that the assimilation efficiency of the protists for iron actually declined when grazing on iron-limited bacteria. They postulated that this was a result of the iron being bound very tightly by the iron-limited bacterial cells, in complexes unavailable to the protists. Regeneration of trace metals as organic complexes by protozoan grazers has been observed in other studies. Twiss and Campbell, 1995, found that $\mathrm{Zn}$ and $\mathrm{Cd}$ regenerated from cyanobacteria cells through protozoan grazing was less available to phytoplankton uptake than inorganic $\mathrm{Zn}$ and $\mathrm{Cd}$, implying organic complexation of the regenerated metals.

Aggregation - In series \#2, the results of the Paraphysomonas grazing culture (Part B) were very different from the Cafeteria grazing culture (Part A). In the Paraphysomonas grazing culture very little regeneration of the radiotracers into the dissolved phase occurred, with the exception of ${ }^{51} \mathrm{Cr}$. About $75 \%$ of the total ${ }^{234} \mathrm{Th}$ and ${ }^{59} \mathrm{Fe}$, and $45 \%$ of the total ${ }^{51} \mathrm{Cr}$ in the grazing cultures ended up in the $>5 \mu \mathrm{m}$ particle size class by $\mathrm{t}_{48}$. This size class also demonstrated a significant increase in organic carbon. Microscopic examination of AO-stained slides from these cultures indicated that the particles in this size class were primarily composed of large, amorphous aggregates of bacteria, possibly combined with other organic material. Factors that may have contributed to the increased aggregation observed in these cultures relative to the Cafeteria grazing cultures include higher levels of DOC in part B, differences in prey surface chemistry, and characteristics 
specific to the grazer Paraphysomonas, such as digestive metabolism and/or quality of waste products.

The control cultures in Part B remained as suspensions of single bacterial cells throughout the course of the experiment, rather than aggregating into clumps as in the grazing culture with Paraphysomonas. This suggests that aggregation was enhanced in some way by the activity of the grazer. Protozoan grazers can facilitate aggregation in several ways. Under grazing pressure, bacteria are known to resort to forming clumps or filaments as a refuge from their predators (Jurgens and Gude, 1994). Given the metabolically active nature of the bacteria in these experiments, this could have contributed to clump formation. Paraphysomonas may also have contributed more directly to aggregation processes by producing surface active particulate or colloidal material. Elevated levels of colloidal material in the Paraphysomonas cultures relative to the Cafeteria cultures are indicated by the higher percentage of colloidal ${ }^{59} \mathrm{Fe}$ and ${ }^{234} \mathrm{Th}$ in the Paraphysomonas cultures (Table 2.3). Protists have been hypothesized to produce colloidal material as a by-product of waste excretion (Nagata and Kirchman, 1992; Tranvik, 1994). Surface active materials produced by the grazer could both enhance aggregation processes, and, by scavenging trace metals, reduce the amount of trace metals regenerated to the dissolved phase.

Other workers studying the cycling of Fe by Paraphysomonas sp. have noticed a tendency for inefficient regeneration of $\mathrm{Fe}$ into the dissolved $(<0.2 \mu \mathrm{m})$ phase. Chase and Price, 1998, observed in their experiments that the percentage of $\mathrm{Fe}$ regenerated into the dissolved phase by Paraphysomonas imperforata dropped by about $80 \%$ in systems without $100 \mu \mathrm{M}$ EDTA present to trap Fe in the dissolved phase. Hutchins and Bruland, 1994, in their experiments with Paraphysomonas sp. (without EDTA) observed that only $6 \%$ of the added Fe radiotracer was regenerated into the dissolved phase in the grazing culture.

Colloidal/dissolved partitioning of trace metals - In both Part A' and Part B of experimental series $\# 2,{ }^{51} \mathrm{Cr}$ demonstrated substantially less partitioning into the colloidal 
size class (defined as $<0.2 \mu \mathrm{m}$ and $>10,000 \mathrm{MWCO}$ ) than either ${ }^{59} \mathrm{Fe}$ or ${ }^{234} \mathrm{Th}$ (Table 2.3). The greater proportion of $\mathrm{Cr}$ in the truly dissolved phase relative to $\mathrm{Fe}$ and $\mathrm{Th}$ may be due to the tendency of $\mathrm{Cr}$ (III) to form extremely stable and inert complexes with dissolved organic ligands, as a result of its slow ligand exchange kinetics (Mayer, 1988). This tendency to complex strongly with dissolved ligands might prevent $\mathrm{Cr}$ from interacting with binding sites on colloid surfaces, thus stabilizing $\mathrm{Cr}$ in the dissolved phase.

Other researchers have found evidence for the differential partitioning of protozoanregenerated trace metals into colloidal and dissolved phases, notably Twiss and Campbell, 1995, and Twiss et. al., 1996. These workers studied the regeneration of ${ }^{109} \mathrm{Cd}$ and ${ }^{65} \mathrm{Zn}$ from radiolabeled Synechococcus cells by protozoan grazers in freshwater. In both a laboratory and a field study, Twiss et. al. found that regenerated $\mathrm{Zn}$ partitioned more strongly into the colloidal phase (as defined by a $5000 \mathrm{MWCO}$ membrane) than Cd. This was interpreted as being related to the cellular localization of $\mathrm{Zn}$ and $\mathrm{Cd}$ within the prey, with $\mathrm{Zn}$ possibly being associated with material of higher molecular weight than $\mathrm{Cd}$, and being regenerated as such due to protozoan grazing. While this explanation is plausible for such micronutrient/nutrient analog trace metals as $\mathrm{Zn}$ and $\mathrm{Cd}$, it is less applicable to explain the difference observed in our studies between metals like $\mathrm{Cr}$ and Th, which are not nutrients and are associated fairly non-specifically with cells. A more likely explanation in this case is the lower particle reactivity of $\mathrm{Cr}$ relative to $\mathrm{Th}$ (and $\mathrm{Fe}$ ).

This explanation of our data also suggests an alternative interpretation of the results of Twiss et. al.: Rather than being a result of different intracellular partitioning of $\mathrm{Zn}$ and $\mathrm{Cd}$, their data showing a higher percentage of regenerated $\mathrm{Zn}$ associated with the colloidal phase may instead be due to the somewhat higher particle reactivity of $\mathrm{Zn}$ relative to $\mathrm{Cd}$. $\mathrm{Zn}$ has a higher ratio of charge to ionic radius, a higher stability constant of the first hydrolysis product, and a much greater affinity for organic complexation than Cd (Morel and Hering, 1993). All of these factors could contribute to give $\mathrm{Zn}$ a higher affinity for the colloidal phase than $\mathrm{Cd}$. 
Metal/Carbon Ratios - In the final part of this discussion, the cycling of trace metals and organic carbon in experimental series \#2 will be examined by looking at how the metalto-carbon ratio of various particulate size classes changed with time in the control and grazing cultures.

Figure 2.14 shows the metal/carbon ratios for ${ }^{59} \mathrm{Fe},{ }^{234} \mathrm{Th}$, and ${ }^{51} \mathrm{Cr}$ in the $>0.2 \mu \mathrm{m}$ size class (sum of all size classes $>0.2 \mu \mathrm{m}$ ) of the control and Cafeteria grazing cultures in Part A. In the control, the pattern for all of the metals was much the same. There was an initial drop in the metal/carbon ratio, most likely due to DOC uptake and, for ${ }^{234} \mathrm{Th}$, metal desorption. The controls reached equilibrium by between 12 to 24 hours, and thereafter a constant metal/carbon ratio was observed. In the grazing cultures, there was a distinctly different pattern. After an initial drop, there was an increase in the $\mathrm{Fe} / \mathrm{C}$ and $\mathrm{Th} / \mathrm{C}$ ratios in the grazing cultures relative to the control. The opposite pattern was observed for $\mathrm{Cr} / \mathrm{C}$ ratios, which decreased relative to the control. Changes in the $\mathrm{Th} / \mathrm{C}$ and $\mathrm{Cr} / \mathrm{C}$ ratios over time in the grazing culture for the $0.2-1 \mu \mathrm{m}$ and $>5 \mu \mathrm{m}$ size classes are shown in Figure 2.15. The pattern in the whole particle pool observed for Th was apparently largely driven by the $>5 \mu \mathrm{m}$ size class, which showed a substantial increase in the $\mathrm{Th} / \mathrm{C}$ ratio. The $\mathrm{Cr} / \mathrm{C}$ ratio in this size class did not increase.

Several processes could potentially account for the changes observed in metal/carbon ratios in the Cafeteria experiment. Increases in the $\mathrm{Th} / \mathrm{C}$ and $\mathrm{Fe} / \mathrm{C}$ ratio of larger particles in the grazing culture could have been driven by preferential retention (bioaccumulation) of $\mathrm{Th}$ and $\mathrm{Fe}$ in protozoan biomass, and remineralization of organic carbon. $\mathrm{Cr} / \mathrm{C}$ ratios might differ from this pattern because $\mathrm{Cr}$ was less available for bioaccumulation than $\mathrm{Th}$ or Fe. One argument against this hypothesis is that microscopic examination indicated that the $>5 \mu \mathrm{m}$ size class was composed primarily of bacterial aggregates, not protozoan biomass.

Another process that might contribute to the increase in $\mathrm{Th} / \mathrm{C}$ and $\mathrm{Fe} / \mathrm{C}$ ratios in larger particles is an increase in the reactivity of particle surfaces, which could lead to increased scavenging of particle reactive $\mathrm{Th}$ and $\mathrm{Fe}$. $\mathrm{Cr}$ might be less affected by such processes because, as discussed previously, it is apparently less surface active than $\mathrm{Th}$ and $\mathrm{Fe}$, 

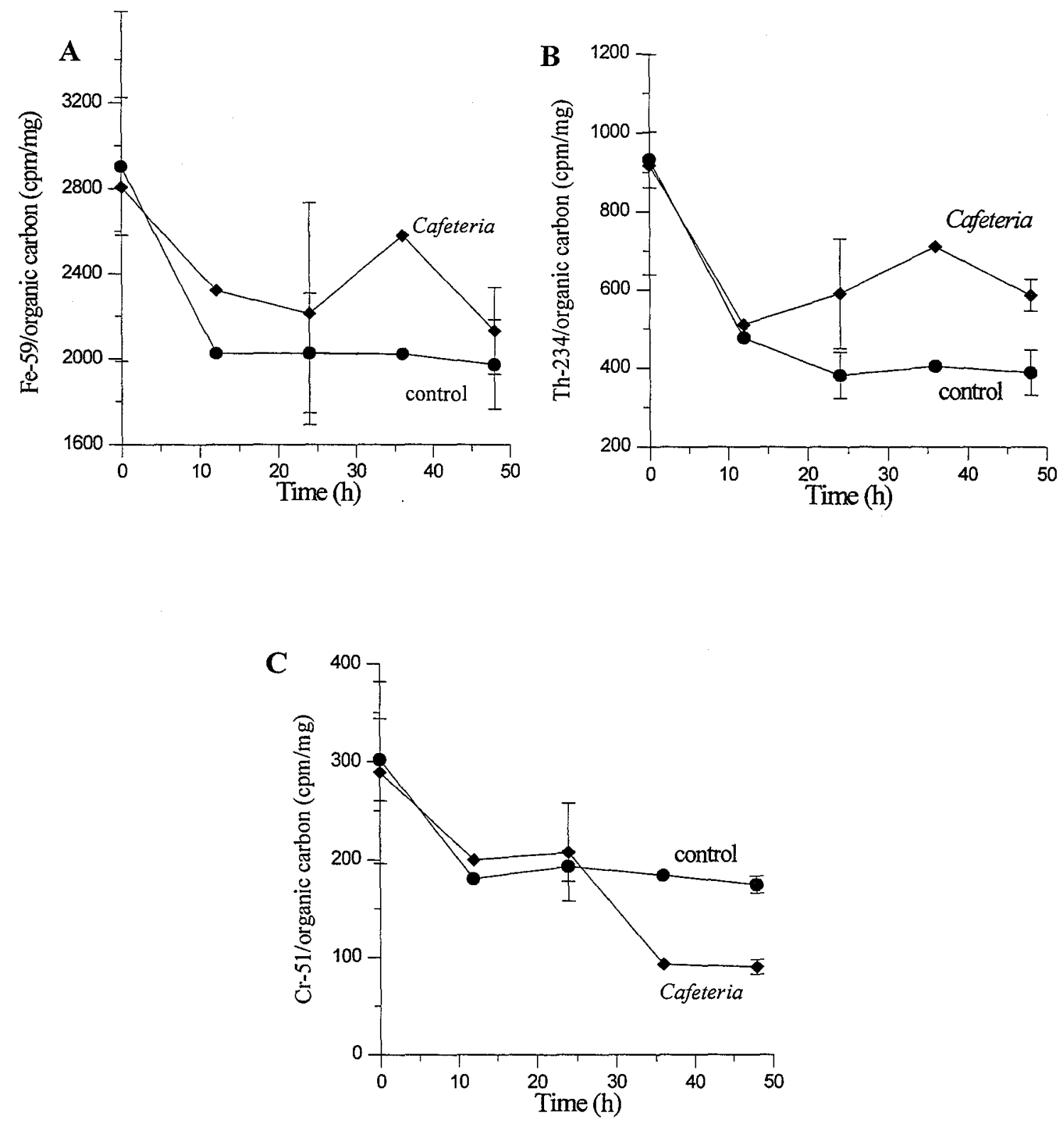

Figure 2.14 - Experimental series \#2, Part A - Cafeteria experiment Metal/Carbon ratios in total particle assemblage $->0.2 \mathrm{um}$

$\mathrm{A}: \mathrm{Fe}-59 / \mathrm{C}$ ratios vs time

B: Th-234/C ratios vs time

C: $\mathrm{Cr}-51 / \mathrm{C}$ ratios vs time 

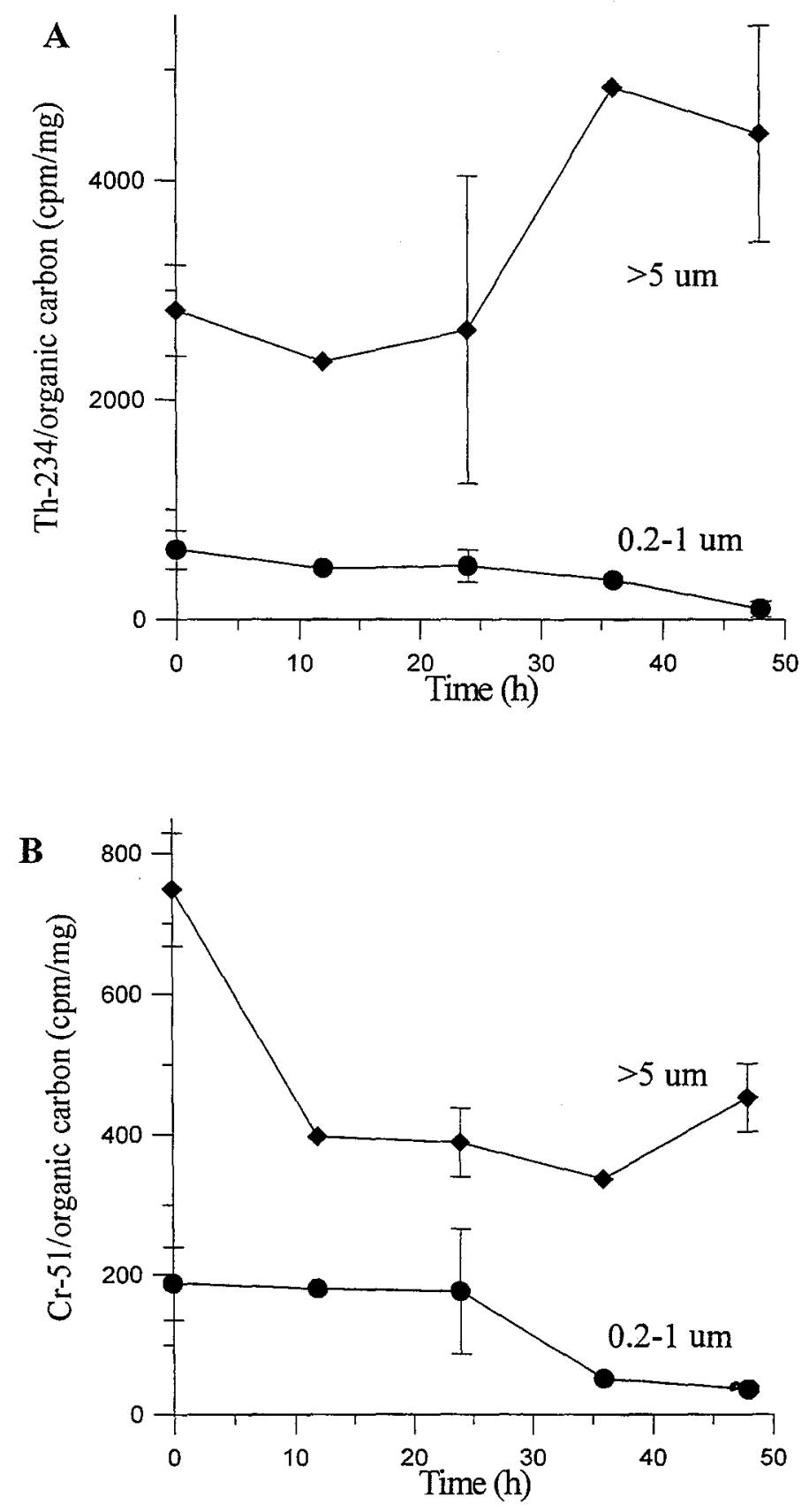

Figure 2.15 - Experimental series \#2, Part A, Cafeteria cultures: Metal/Carbon ratios in small and large particle size class A: Th-234/C ratio in $0.2-1$ um and $>5$ um size class vs time $\mathrm{B}$ : $\mathrm{Cr}-51 / \mathrm{C}$ ratio in $0.2-1 \mathrm{um}$ and $>5$ um size class vs time 
possibly due to organic complexation. Protozoan waste products may have a surface chemistry substantially different from the original bacterial prey particles, facilitating enhanced metal scavenging and aggregation in culture. If this were the only process affecting metal/carbon ratios, however, increases in the metal/carbon ratio would most likely be observed in small as well as large particle size classes.

Protozoan waste products may include colloidal material (Nagata and Kirchman, 1992; Tranvik, 1994). Sorption of particle-reactive trace metals (including Th) to colloidal phases has been postulated to be instrumental in the transfer of these trace metals to larger size classes, through the phenomenon of "colloidal pumping" - i.e. sorption of trace metals to colloidal phases followed by aggregation of colloids with larger particles (Honeyman and Santschi 1989; Honeyman and Santschi 1991; Moran and Buesseler 1992). Colloids, with their high surface area to volume ratios, should have high $\mathrm{Th} / \mathrm{C}$ and $\mathrm{Fe} / \mathrm{C}$ ratios relative to larger particles. If colloids produced as a consequence of protozoan grazing scavenge grazer-regenerated $\mathrm{Th}$ and $\mathrm{Fe}$ and then coagulate with bacteria to form larger, aggregated particles, it should result in an increase in the $\mathrm{Th} / \mathrm{C}$ and $\mathrm{Fe} / \mathrm{C}$ ratio of the large particle size class relative to the beginning of the experiment, when aggregates larger than $5 \mu \mathrm{m}$ were fewer (confirmed by microscopy), and did not incorporate colloids generated by the grazers. The pattern observed for $\mathrm{Cr} / \mathrm{C}$ would differ because $\mathrm{Cr}$ has less of a tendency than $\mathrm{Fe}$ and $\mathrm{Th}$ to partition onto colloids (Table 2.3). This mechanism may account for the metal/carbon ratios observed in the Cafeteria experiment. It may also explain why $\mathrm{Cr}$ demonstrated less of a tendency (relative to $\mathrm{Fe}$ and $\mathrm{Th}$ ) to partition into the $1-5 \mu \mathrm{m}$ or $>5 \mu \mathrm{m}$ particle size class in the Paraphysomonas grazing culture: The lower affinity of $\mathrm{Cr}$ for colloids might have kept it from being aggregated up the particle size class spectrum to the same extent as Fe and Th.

Given this hypothesis, large increases in $\mathrm{Th} / \mathrm{C}$ and $\mathrm{Fe} / \mathrm{C}$ ratios in the Paraphysomonas grazing cultures might be expected, since concentrations of $\mathrm{Fe}$ and $\mathrm{Th}$ in the colloidal phase were elevated relative to the Cafeteria experiment (Table 2.3), and more extensive aggregation occurred. Contrary to expectations, however, metal/carbon ratios did not increase in the $>0.2 \mu \mathrm{m}$ size class of the Paraphysomonas grazing cultures (Figure 2.16). 

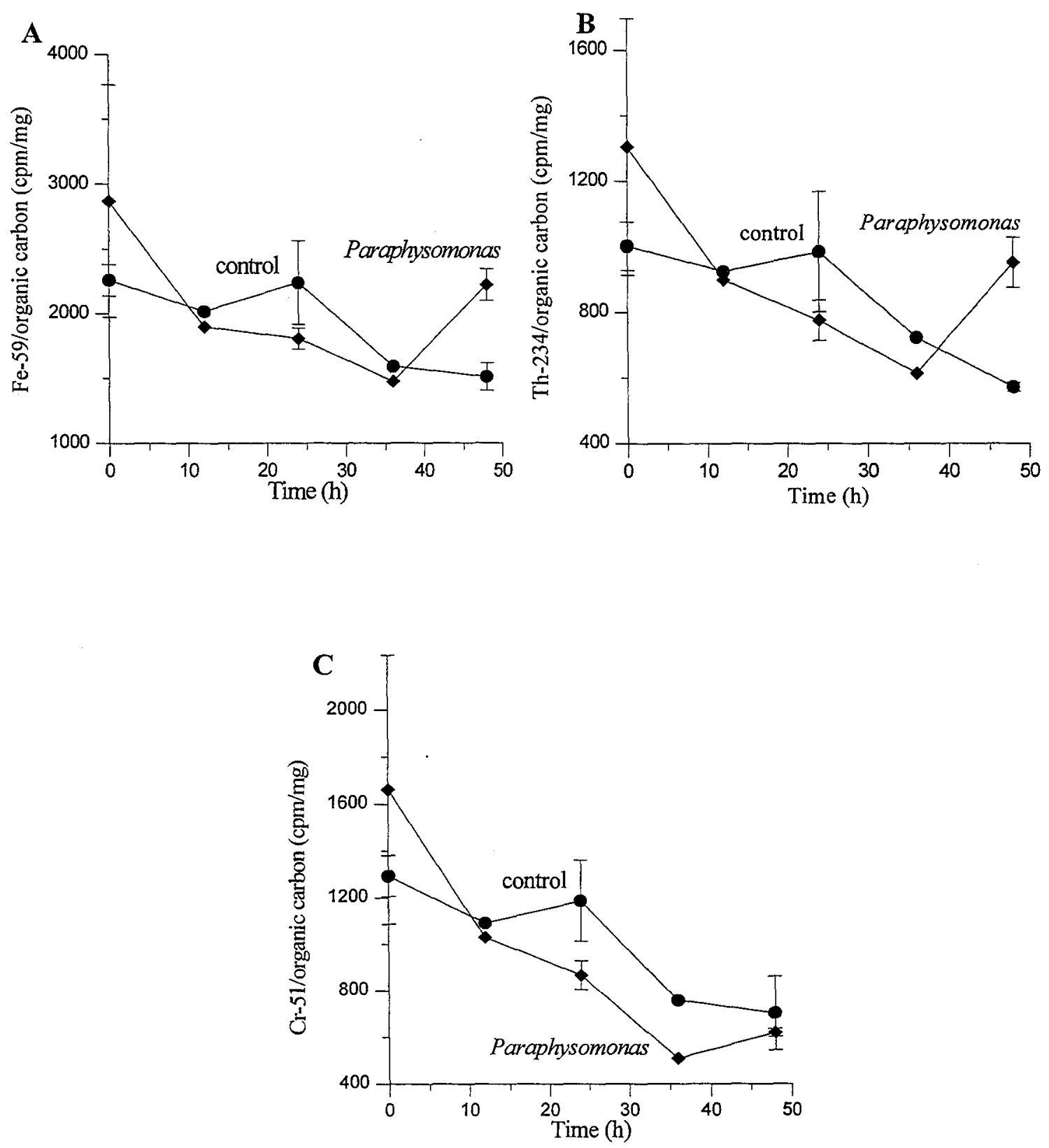

Figure 2.16 - Experimental series \#2, Part B - Paraphysomonas experiment Metal/Carbon ratios in total particle assemblage $->0.2 \mathrm{um}$

A: $\mathrm{Fe}-59 / \mathrm{C}$ ratios vs time

B: $\mathrm{Th}-234 / \mathrm{C}$ ratios vs time

C: $\mathrm{Cr}-51 / \mathrm{C}$ ratios vs time 
The general pattern for ${ }^{59} \mathrm{Fe},{ }^{234} \mathrm{Th}$, and ${ }^{51} \mathrm{Cr}$, in both control and experimental cultures, was one of decreasing metal/carbon ratios.

Metal/carbon ratios could be decreased by desorption of trace metals from particles into the dissolved phase. Desorption was shown not to occur, however, in the Paraphysomonas experiment. The controls, for example, demonstrated little to no desorption of ${ }^{234} \mathrm{Th}$, yet ${ }^{234} \mathrm{Th} / \mathrm{C}$ ratios decreased. Another means of decreasing metal/carbon ratios would be increasing particle size, which would reduce surface area to volume ratios and thus decrease metal/carbon ratios. Along with increasing particle size, taking up organic carbon (increasing POC) would also decrease metal/carbon ratios. One mechanism to increase POC and decrease metal/carbon ratios in both the control and experimental cultures in the Paraphysomonas experiment would be uptake of the high $(13 \mathrm{mg} / \mathrm{l})$ concentrations of DOC present in that experiment. As shown in Fig. 2.10 (C and D), there was uptake of DOC in both the experimental and control cultures. Comparing DOC to TOC in the Paraphysomonas experiment (Fig. $2.10 \mathrm{C}$ and D and Table 2.1), there was an increase in POC from about $5 \mathrm{mg} / 1$ at $\mathrm{t}_{0}$ to $10 \mathrm{mg} / \mathrm{l}$ at $\mathrm{t}_{48}$ in both control and grazing cultures in that experiment. This increase in POC most likely accounts for the observed decrease in metal/carbon ratios in the Paraphysomonas experiment.

It was only at the very end of the Paraphysomonas grazing cultures, when DOC levels had been reduced to near background, that an increase in the $\mathrm{Fe} / \mathrm{C}$ and $\mathrm{Th} / \mathrm{C}$ ratios (but not in the $\mathrm{Cr} / \mathrm{C}$ ratio) was observed (Fig. 2.16). This may have been due to aggregation of $\mathrm{Th}$ and $\mathrm{Fe}$ associated with colloidal material into larger size classes, as postulated for the Cafeteria experiment. A look at the $\mathrm{Th} / \mathrm{C}$ and $\mathrm{Cr} / \mathrm{C}$ dynamics of individual particle size classes in the Paraphysomonas culture (Figure 2.17), demonstrates a very large peak in the metal/carbon ratio of the $>5 \mu \mathrm{m}$ size class for both Th and Cr. This is probably artificial, caused by the fact that the non-labeled cultures from which the organic carbon numbers were taken were slightly out of phase with the radiolabeled cultures at $t_{36}$ (see Figs. 2.10 and 2.11). 

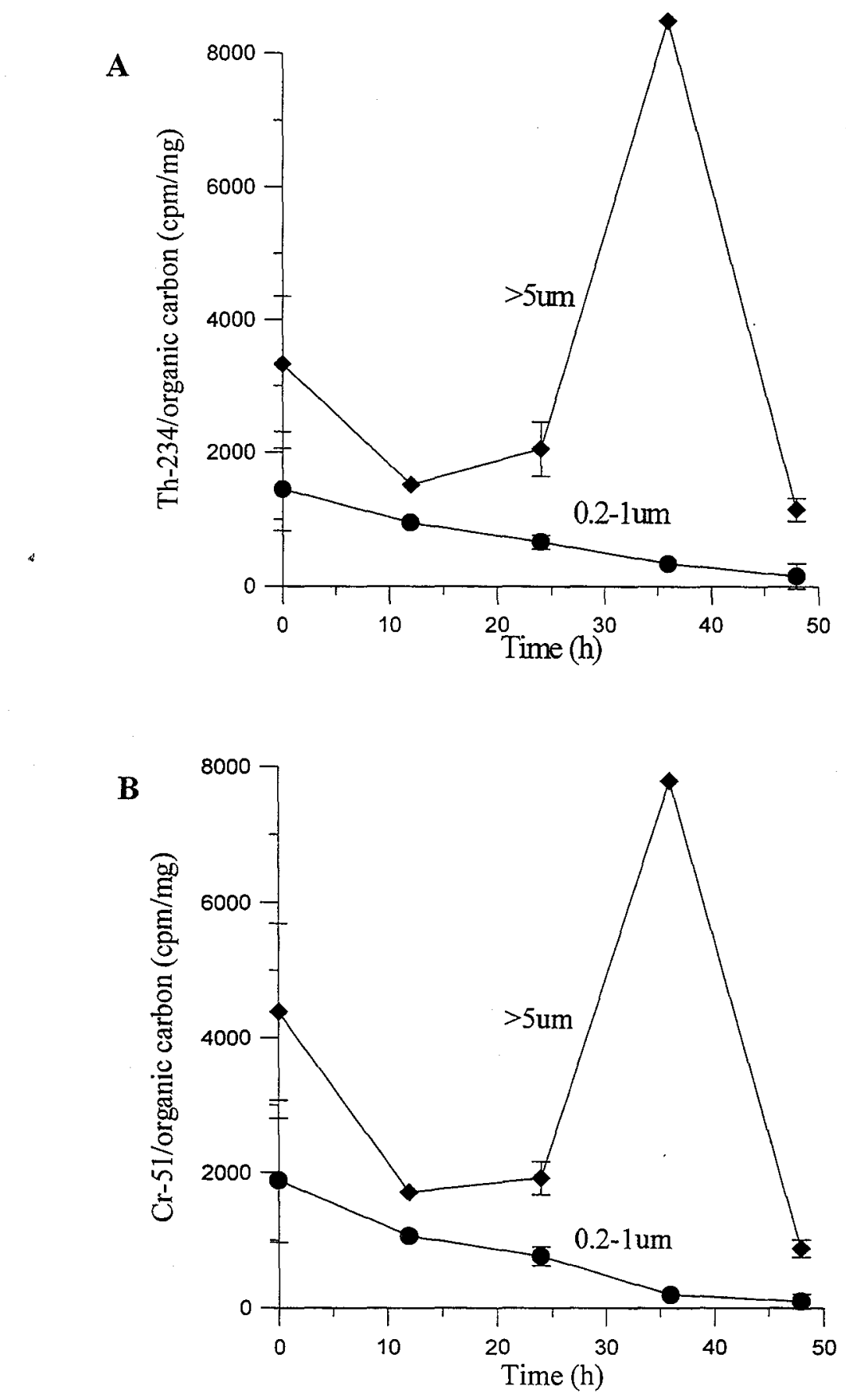

Figure 2.17 - Experimental series \#2, Part B, Paraphysomonas cultures: Metal/Carbon ratios in small and large particle size class A: Th-234/C ratio in $0.2-1$ um and $>5$ um size class vs time B: $\mathrm{Cr}-51 / \mathrm{C}$ ratio in $0.2-1 \mathrm{um}$ and $>5$ um size class vs time 
In summary, several processes could affect the metal/carbon ratios in these model grazing systems. Remineralization of organic carbon by grazers and bioaccumulation of trace metals would tend to increase metal/carbon ratios. Increasing particle size by increasing POC would decrease particle surface area to volume ratios, and thereby decrease metal/carbon ratios. Changes in the surface reactivity of particles could drive metal/carbon ratios either up or down, depending on how particle surface reactivity changes. Physical processes of aggregation can also affect metal/carbon ratios by moving small particles and colloids up the particle size spectrum. The preceding analysis of metal/carbon ratios in experimental series \#2 cannot prove or disprove the significance of any of these processes in contributing to the patterns observed, but some tentative conclusions are possible. While metal/carbon ratios in the Paraphysomonas experiment were apparently dominated by DOC-POC dynamics, results from the Cafeteria experiment suggest that colloids can be a key factor in determining the fate of trace metals with respect to organic carbon in grazing cultures.

Recent models of Th cycling in the ocean have considered the colloidal pool and demonstrated its importance to Th/particle dynamics (Honeyman and Santschi, 1989; Moran and Buesseler 1992; Moran et. al. 1993). Our results indicate that, as mediated by the microbial loop, partitioning of Th into the colloidal phase and subsequent aggregation of colloids with larger particles can increase the Th/carbon ratio of aggregated particles in the system. A recent compilation of data on ${ }^{234} \mathrm{Th} / \mathrm{POC}$ values obtained for different size classes of particles in field studies indicates that ${ }^{234} \mathrm{Th} / \mathrm{POC}$ ratios from various locations increase with increasing particle size (Burd et. al., 1998). Biological recycling of Th may impact $\mathrm{Th} /$ carbon ratios differently depending on the particular regime involved, i.e. dominance of microbial loop processing vs. new production/high particle flux (eg. Moran et. al., 1993). The results obtained in this chapter on the cycling of Th and organic carbon as mediated by protozoan grazers in model systems may contribute to our understanding of local variations in Th/carbon ratios observed in field studies of ${ }^{234} \mathrm{Th}$ as a tracer for organic carbon flux out of the euphotic zone (Buesseler et. al., 1992, 1995; Murray et. al. 1996). 


\section{Conclusion}

\section{Summary}

The work presented in this chapter has contributed to our growing understanding of how protozoan grazers mediate the biogeochemical cycling of particle-reactive trace metals in marine systems. Principle results can be summarized as follows:

- Protozoans appear to be able to mobilize trace metals bound extracellularly just as effectively as those contained intracellularly.

- In some cases, protozoan regeneration of trace metals from prey appears to stabilize otherwise particle-reactive trace metals like $\mathrm{Th}$ and $\mathrm{Fe}$ in the dissolved phase, possibly through the formation of metal-organic complexes.

- Protozoan grazing may generate colloidal material, which may scavenge particlereactive trace metals and aggregate with larger particles, resulting in an increase in the metal/carbon ratio of larger particle size classes.

- The degree to which protozoan grazing leads to the formation of colloidal trace metals and enhanced aggregation processes is affected by several factors, including prey surface chemistry and characteristics of protozoan metabolism.

\section{Application to Natural Systems}

It is not possible to extrapolate directly from these laboratory experiments into the natural environment. Due to the higher concentrations of protists and prey cells in our model systems, the rate and magnitude of the processes therein are elevated relative to what they would be in more dilute natural systems. The basic mechanisms, however, remain the same regardless of concentration. Work like this, using model systems, serves to identify processes and the mechanisms behind them. Further work is needed in order to identify the the relative importance of these processes and mechanisms in natural systems. 
It is likely that, in the water column of the open ocean, with protists grazing on single bacterial cells in suspension, the kinds of aggregation processes that we have observed in our systems would be much less important, or even non-existent. However, consumption of particle-reactive trace metals associated with prey cells by protozoan grazers should still result in the formation of colloidal and organically-complexed forms of these trace metals. Because this changes trace metal bioavailability and/or particle reactivity, this process will have an important impact on trace metal cycling.

With regard to aggregation processes, it is important to remember that protists occur in a variety of aquatic environments, most of them more concentrated than the oceanic water column. Even within the open ocean, particle-rich microenvironments like marine snow have been shown to be important in the cycling of materials and energy in oceanic systems (Honjo et. al. 1984; Pilskaln and Honjo 1987; Knauer et. al. 1982; Alldredge et. al., 1993). Protozoan grazers are known to be an abundant and important component of such microenvironments (Caron et. al., 1986; Alldredge and Silver, 1988). It is likely that in such microenvironments, protozoan-mediated aggregation processes of the type identified in our model systems play an important role in physico-chemical processes that affect trace metal speciation and transport. The same is true for other environments that are relatively high in both particle and protozoan abundance - coastal systems, estuaries, and air/sea and sediment/water interfaces.

\section{Future Work}

As is often the case, this attempt to answer questions and eliminate possibilities through experimental work has opened up further possibilities and new lines of inquiry. This work has confirmed the results of previous workers in showing that protists can regenerate trace metals from prey cells into the dissolved phase. For particle-reactive trace metals, depending on various factors, regeneration of metals to the colloidal phase with subsequent aggregation can be just as, or even more important, consequences of protozoan grazing. 
Future work could focus on the factors that control the ultimate speciation of trace metals regenerated from prey cells by protozoan grazers. These factors include protozoan metabolism (as dependent on protozoan species, growth phase, nature of prey, prey concentration, etc.); prey surface chemistry (as dependent on prey species, nutrient limitation status, growth phase, etc.); particle concentration; DOC concentration; and trace metal chemistry. It is apparent that numerous areas remain open to further research.

In the short term, I have plans to pursue the question of protist-specific metabolism by revisiting the comparison between Paraphysomonas and Cafeteria, this time conducting simultaneous grazing studies on the same batch of bacterial prey. I also plan to track bacterial carbon in addition to trace metals by using bacteria uniformly labeled with ${ }^{14} \mathrm{C}$ as well as trace metal radionuclides. This, along with measurements of stable DOC throughout the time course of the experiment, will provide a more complete understanding of the carbon cycling in the system. Finally, I would like to characterize the speciation of protozoan-regenerated trace metals in some more detail, particularly with regard to the strength of organic complexation (if any). 


\section{References}

- Alldredge, A.L. and M.W. Silver. 1988. Characteristics, dynamics and significance of marine snow. Prog. Oceanogr. 20: 41-82.

- Alldredge, A.L., U. Passow and B.E. Logan. 1993. The abundance and significance of a class of large, transparent organic particles in the ocean. Deep-Sea Res. 40: 1131-1140.

- Azam, F., D.C. Smith and A.F. Carlucci. 1992. Bacterial transformation and transport of organic matter in the Southern California Bight. Prog. Oceanog. 30: 151-166.

- Azam, F., T. Fenchel, J.G. Field, L.A. Meyer-Reil and F. Thingstad. 1983. The ecological role of water-column microbes in the sea. Mar. Ecol. Prog. Ser. 10: 257-263.

- Bruland, K.W. 1983. Trace elements in seawater. p. 157-220. In Chemical Oceanography, Vol. 8. Riley, J.P and R. Chester, eds. Academic Press, London.

- Buesseler, K.O., M.P. Bacon, J.K. Cochran and H.D. Livingston. 1992. Carbon and nitrogen export during the JGOFS North Atlantic Bloom Experiment estimated from ${ }^{234} \mathrm{Th}:{ }^{238} \mathrm{U}$ disequilibria. Deep-Sea Res. 39: 1115-1137.

- Buesseler, K.O., J.A. Andrews, M.C. Hartman, R. Belastock and F. Chai. 1995. Regional estimates of the export flux of particulate organic carbon derived from thorium-234 during the JGOFS EqPac program. Deep-Sea Res. 42: 777-804.

- Burd, A., S.B. Moran and G.A. Jackson. 1998. A coupled adsorption-aggregation model of the $\mathrm{POC} /{ }^{234} \mathrm{Th}$ ratio of marine particles. Deep-Sea Res. in press.

- Caron, D.A., P.G. Davis, L.P. Madin and J.McN. Sieburth. 1986. Enrichment of microbial populations in macroaggregates (marine snow) from surface waters of the North Atlantic. J. Mar. Res. 44: 543-565.

- Chase, Z. and N.M. Price. 1998. Metabolic consequences of iron deficiency in heterotrophic marine protozoa. Limnol. Oceanogr. in press.

- Clegg, S.L. and J.L. Sarmiento. 1989. The hydrolytic scavenging of metal ions by marine particulate matter. Progress in Oceanography 23: 1-21.

- Clegg, S.L. and M. Whitfield. 1991. A generalized model for the scavenging of trace metals in the open ocean - II. Thorium scavenging. Deep-Sea Res. 38: 91-120. 
- Coale, K.H. and K. W. Bruland. 1987. Oceanic stratified euphotic zone as elucidated by ${ }^{234} \mathrm{Th}:{ }^{238} \mathrm{U}$ disequilibria. Limnol. Oceanogr. 32: 189-200.

- Cowen, J.P. 1991. Individual particle analysis. p.429-436. In Marine Particles: Analysis and Characterization. Hurd, D.C. and D.W. Spencer, eds. Geophysical Monograph 63. AGU, Washington, D.C.

- Decho, A.W. and S.N. Luoma. 1991. Time-courses in the retention of food material in the bivalves Potamocorbula amurensis and Macoma balthica: significance to the absorption of carbon and chromium. Mar. Ecol. Prog. Ser. 78: 303-314.

- Decho, A.W. and S.N. Luoma. 1994. Humic and fulvic acids: sink or source in the availability of metals to the marine bivalves Macoma balthica and Potamocorbula amurensis? Mar. Ecol. Prog. Ser. 108: 133-145.

- Fenchel, T. 1987. Ecology of Protozoa: The Biology of Free-living Phagotrophic Protists. Madison: Science Tech Publishers.

- Fok, A.K., J.H. Muraoka and R. D. Allen. 1984. Acid phosphatase in the digestive vacuoles and lysosomes of Paramecium caudatum: A timed study.J. Protozool. 31: 216-220.

- Fok, A.K., Y. Lee and R.D. Allen. 1982. The correlation of digestive vacuole $\mathrm{pH}$ and size with the digestive cycle in Parmecium caudatum. J. Protozool. 29: 409-414.

- Goldman, J.C. 1984. Conceptual role for microaggregates in pelagic waters. Bull. Mar. Sci. 35: 462-476.

- Gustafsson, O., P.M. Gschwend and K.O. Buesseler. 1997. Using ${ }^{234}$ Th disequilibria to estimate the vertical removal rates of polycyclic aromatic hydrocarbons from the surface ocean. Mar. Chem. 57: 11-23.

- Hobbie, J.E, R. Daley and S. Jasper. 1977. Use of Nuclepore filters for counting bacteria by fluorescence microscopy. Appl. Environ. Microbiol. 33: 1225-1228.

- Honeyman, B.D. and P.H. Santschi. 1989. A Brownian-pumping model for oceanic trace metal scavenging: Evidence from Th isotopes. J. Mar. Res. 47: 951-992. 
- Honeyman, B.D. and P.H. Santschi. 1991. Coupling adsorption and particle aggregation: Laboratory studies of "colloidal pumping" using ${ }^{59} \mathrm{Fe}$-labeled hematite. Environ. Sci. Technol. 25: 1739-1747.

- Honjo, S., D.W. Doherty, Y.C. Agarwal and V.Asper. 1984. Direct optical assessment of large amorphous aggregates (marine snow) in the deep ocean. Deep-Sea Res. 31: 6776.

- Hudson, R.J.M. and F.M.M. Morel. 1989. Distinguishing between extra- and intracellular iron in marine phytoplankton. Limnol. Oceanogr. 34: 1113-1120.

- Hutchins, D.A. and K.W. Bruland. 1994. Grazer-mediated regeneration and assimilation of Fe, $\mathrm{Zn}$ and $\mathrm{Mn}$ from planktonic prey. Mar. Ecol. Prog. Ser. 110: 259-269.

- Jurgens, K. and H. Gude. 1994. The potential importance of grazing-resistant bacteria in planktonic systems. Mar. Ecol. Prog. Ser. 112: 169-188.

- Knauer, G.H., D. Hebel and F. Cipriano. 1982. Marine snow: major site of primary production in coastal waters. Nature, Lond. 300: 630-631.

- Landry, M.R. and others. 1997. Iron and grazing constraints on primary production in the central equatorial Pacific: An EqPac synthesis. Limnol. Oceanogr. 42: 405-418.

- Lee, J.J. and G.M. Capriulo. 1990. The ecology of marine protozoa: An overview. p. 339. In Ecology of Marine Protozoa. Capriulo, G.M. ed. Oxford University Press, New York.

- Lopez, G.R., P. Tantichodok and I.-J. Cheng. 1989. Radiotracer methos for determining utilization of sedimentary organic matter by deposit feeders. P. 149-170. In Lopez, G.R. et. al., eds. Ecology of marine deposit feeders, V. 31. Springer.

- Margerum, D.W., G.R. Cayley, D.C. Weatherburn and G.K. Pagenkopf. 1978. Coordination chemistry. p. 1-220. In ACS Monograph 20. Martell, A.E., ed. Washington, D.C.

- Martin J.H. and S.E. Fitzwater. 1988. Iron deficiency limits phtoplankton growth in the north-east Pacific subarctic.Nature. 331:341-343. 
- Mayer, L.M. 1988. Geochemistry of chromium in the oceans. p. 173-188. In Chromium in the Natural and Human Environments. Nriagu, J.O. and E. Nieboer, eds. Wiley Advances in Environmental Science and Technology Series, 20.

- Mertz, W. 1969. Chromium occurrence and function in biological systems. Physiol. Rev. 49: 163-239.

- Moran, S.B. and Buesseler, K.O. 1992. Short residence time of colloids in the upper ocean estimated from ${ }^{234} \mathrm{Th}^{238} \mathrm{U}$ disequilibria. Nature 359: 221-223.

- Moran, S.B., K.O. Buesseler, S.E.H. Niven, M.P. Bacon, J.K. Cochran, H.D. Livingston and A.F. Michaels.1993. Regional variability in size fractionated C/234Th ratios in the upper ocean: Importance of biological recycling. The third Scientific Meeting of the Oceanography Society, Seattle, WA; April 13-16; published program, p. 152.

- Morel, F.M.M. and J.G. Hering. 1993. Principles of Aquatic Chemistry. WileyInterscience, New York.

- Murray, J.W. and others. 1996. Export flux of particulate organic carbon from the central equatorial Pacific determined using a combined drifting trap $-{ }^{234} \mathrm{Th}$ approach. Deep-Sea Res. 43: 1095-1132.

- Nagata, T. and D.L. Kirchman. 1990. Filtration-induced release of dissolved free amino acids: application to cultures of marine protozoa. Mar. Ecol. Prog. Ser. 68: 1-5.

- Nagata, T. and D.L. Kirchman. 1992. Release of macromolecular organic complexes by heterotrophic marine flagellates. Mar. Ecol. Prog. Ser. 83: 233-240.

- Nieboer, E. and A.A. Jusys. 1988. Biologic chemistry of chromium. p. 21-80. In Chromium in the natural and human environments. Nriagu, J.O and E. Nieboer, eds. Wiley Advances in Environmental Science and Technology, 20.

- Pilskaln, C.H. and S. Honjo. 1987. The fecal pellet fraction of biogeochemical particle fluxes to the deep sea. Global biogeochem. Cycles 1: 31-48.

- Pomeroy, L.R. 1974. The ocean's food web, a changing paradigm. Bioscience 24: 499504.

- Reinfelder, J.R. and N.S. Fisher. 1991. The assimilation of elements ingested by marine copepods. Science 251: 794-796. 
- Riley, G.A. 1963. Theory of food chain relations in the ocean. P. 438-463. In The Sea,Vol. 2. Hill, M.N. ed. Interscience.

- Rue, E.L. and K. W. Bruland. 1995. Complexation of iron(III) by natural organic ligands in the Central North Pacific as determined by a new competitive ligand equilibration/adsorptive cathodic stripping voltammetric method. Mar. Chem. 50: 117138.

- Sherr, E. and B. Sherr. 1988. Role of microbes in pelagic food webs: A revised concept. Limnol. Oceanogr. 33: 1225-1227.

- Sieburth, J.McN. 1984. Protozoan bacterivory in pelagic marine waters. p. 405-444. In Heterotrophic Activity in the Sea. Hobbie, J.E. and P.J.LeB. Williams, eds. Plenum Press, NY.

- Tranvik, L. 1994. Colloidal and dissolved organic matter excreted by a mixotrophic flagellate during bacterivory and autotrophy. Appl. Env. Microbiol. 60: 1884-1888.

- Twiss, M.R. and P.G.C. Campbell. 1995. Regeneration of trace metals from picoplankton by nanoflagellate grazing. Limnol. Oceanogr. 40: 1418-1429.

- Twiss, M.R., P.G.C. Campbell and J.-C. Auclair. 1996. Regeneration, recycling and trophic transfer of trace metals by microbial food-web organisms in the pelagic surface waters of Lake Erie. Limnol. Oceanogr. 41: 1425-1437.

- Valiela, I. 1995. Marine Ecological Processes. Springer, New York.

- Vonk, H.J., 1960. Digestion and metabolism. p.291-313. In The Physiology of Crustacea. Waterman, T.H. ed. Academic Press, New York and London.

- Wells, M.L., N.M. Price and K.W. Bruland. 1995. Iron chemistry in seawater and its relationship to phytoplankton: A workshop report. Mar. Chem. 48: 157-182.

- Wu, J. and G.W. Luther. 1995, Complexation of Fe(III) by natural organic ligandds in the Northwest Atlantic Ocean by a competitive ligand equilibration method and kinetic approach. Mar. Chem. 50: 159-177. 


\section{CHAPTER 3}

\section{Dissolution of colloidal iron oxides by protozoan grazers: Initial results}

\section{Introduction}

\section{General Background}

Since the first evidence for iron limitation of phytoplankton production in high nutrient, low-chlorophyll (HNLC) regions of the world's oceans (Martin and Fitzwater, 1988; Martin and Gordon, 1988), the seawater chemistry of iron and its relationship to phytoplankton have been the subject of intensive research by a number of different groups (see Wells et. al., 1995). At this stage, a decade since Martin's pioneering work in the subarctic North Pacific, there seems little doubt that iron plays an important role in controlling primary productivity in several oceanic regions. Bottle incubation experiments in the equatorial Pacific and in the Southern Ocean, in addition to the subarctic Pacific, have demonstrated the effect of added iron on the phytoplankton communities in these HNLC areas (Martin et. al. 1989, 1990; Johnson et. al., 1994; de Baar et. al. 1990; Van Leeuwe et. al. 1997). These results have been confirmed by mesoscale iron addition experiments in the equatorial Pacific, which documented dramatic increases in phytoplankton biomass, chlorophyll content, and productivity as a result of the addition of low concentrations of dissolved iron to surface waters (Martin et. al., 1994; Coale et. al., 1996).

Despite progress made in proving the importance of iron as a factor in the control of phytoplankton growth, our knowledge of iron biogeochemistry in the oceans is still far from complete. Defining iron bioavailability continues to be an elusive goal for researchers in the field. Our understanding of trace metal bioavailability is founded on the concept of phytoplankton uptake of free metal ions (Sunda and Guillard, 1976; Anderson and Morel 1978); organically complexed and colloidal/particulate trace metal forms are not traditionally thought of as bioavailable. These concepts are not readily applicable to 
iron bioavailability in marine systems, due to the complexity of iron speciation in seawater and due to biological influences on that speciation. Iron(III) is extensively hydrolyzed in oxygenated seawater at $\mathrm{pH} 8$, so its thermodynamically stable speciation state is as an insoluble oxyhydroxide. This speciation is readily influenced, however, by iron(III)/iron(II) redox transitions and organic complexation of iron(III). Recent research suggests that $99.9 \%$ of dissolved iron in ocean surface waters is strongly complexed, by organic ligands thought to be of biological origin (Rue and Bruland 1995; Wu and Luther 1995). The influence of these ligands on iron bioavailablity is unknown.

Iron thus exhibits an array of dissolved, particulate, and colloidal species in marine systems, the bioavailability of many of these forms being ill-defined. In the most general terms, the various forms of iron can be classified as either bioavailable or nonbioavailable. In the literal sense this definition depends not only on the chemical speciation of iron, but also on the form of iron available to specific biological uptake mechanisms, and on the kinetic balance between iron exchange amongst various chemical species, biological uptake, and ecosystem structure/demand (Wells et. al., 1995). Clearly, an absolute and accurate classification of iron as being either bioavailable or non-bioavailable is not possible at the present time. As operationally defined by the techniques available to chemical oceanographers, bioavailable iron usually translates into labile (reactive) iron, as opposed to forms that are non-reactive or inert. In most marine systems, at any given time, the majority of iron present occurs in the non-labile pool, the labile pool being much smaller and more ephemeral due to the dual influences of biological demand and thermodynamic stability.

Mechanisms that mediate the conversion of iron from the non-labile pool to the labile pool in oceanic surface waters are key parameters in iron biogeochemistry. To date, several processes have been described that contribute to the maintenance of the available iron pool: Photoreduction, ligand complexation, enzymatic reactions, and thermal reactions. Work presented in this chapter demonstrates that the grazing activity of heterotrophic protists represents an additional mechanism for the production of reactive 
iron from non-bioavailable forms, due to chemical reactions that take place within the microenvironment of the phagotrophic food vacuole.

\section{Processes influencing iron availability}

Photoreduction - Among known pathways for the conversion of iron from non-reactive into reactive forms, photochemical reduction has been the one most intensively studied (eg. Waite and Morel 1984 a and b; Wells et. al. 1991a; Wells and Mayer 1991a; Johnson et. al. 1994). Photochemical redox cycling of both soluble and synthetic colloidal iron oxides in seawater has been documented. Ligand-to-metal charge transfer reactions are a key component of photochemical iron cycling (Sulzberger and Laubscher, 1995), thus photochemical reaction rates are enhanced by the presence of naturally occuring organic chromophores in seawater. Reaction with secondary photolysis products (indirect photolysis) may be another important photoredox process for iron in seawater (Voelker and Sedlak, 1995). Photoreduction is thought to be an important mechanism for the conversion of colloidal iron to reactive soluble iron (Johnson et. al., 1994; Wells and Mayer, 1991a).

Ligand complexation - Recent findings that $>99 \%$ of the dissolved iron $(<0.2 \mu \mathrm{m})$ in seawater is organically complexed by strong ligands (Rue and Bruland, 1995; Wu and Luther, 1995; van den Berg 1995) has generated interest in the idea of bacteriallyproduced siderophores in seawater. These strong iron-binding ligands are known to be important in the ecology of soil microorganisms and enteric bacteria (Neilands, 1974; Winkelmann et. al., 1987; Matzanke et. al., 1989; Crumbliss, 1991). Marine bacteria have also recently been shown to produce siderophores in culture (Haygood et. al., 1993; Reid et. al., 1993; Wilhelm and Trick, 1994). Although siderophores have not yet been isolated in seawater, the known occurrence of siderophore-like strong binding ligands in ocean waters combined with the production of siderophores by marine bacteria suggest that siderophores may facilitate iron acquisition by bacteria in the oceans. Substantial concentrations of weaker iron-binding ligands have also been shown to exist (Rue and 
Bruland, 1995), and these ligands may also work to keep iron in soluble form. Thus ligand-mediated pathways may be important in the conversion of non-reactive iron into iron that is biologically available. The question of interspecies competition for the uptake of siderophore-bound iron adds another layer of complexity to this hypothesis.

Enzymatic reactions - Recent data suggest that phytoplankton may employ plasmalemma redox proteins to reduce $\mathrm{Fe}$ (III)-organic complexes at the cell surface and thus produce iron available for uptake. This mechanism has been reported from diatom cell cultures (Soria-Dengg and Horstman, 1995) as well as from field populations (M.T. Maldonado, personal communication). Phytoplankton may be able to use this mechanism to access iron bound by microbially-produced siderophores as well as iron bound by synthetic chelators like EDTA (Soria-Dengg and Horstman, 1995). There is no evidence to date that phytoplankton are able to enzymatically reduce colloidal and solid forms of iron at cell surfaces. The work of Maldonado and Price, 1996 indicates that the enzyme nitrate reductase is involved in the extracellular reduction of Fe-EDTA complexes. This mechanism may help cells growing on oxidized $N$ to sustain high growth rates despite higher iron requirements for growth on nitrate vs ammonium. Iron(III) could also potentially be reduced enzymatically by dissolved enzymes secreted by cells or released as a consequence of grazing. Thus far evidence to support this idea has not been presented.

Thermal reactions - Some organic ligands, such as tannic acid, are known to reduce iron(III) upon complexation. Although such reactions could take place in surface seawater, the significance of such processes is thought to be much less than that of photoreductive processes (Wells et. al., 1995), at least in surface waters. Ligand-mediated thermal reduction of iron(III) may be of greater relative importance below the euphotic zone, or in localized regions of high DOC concentration. Thermal dissolution of inorganic iron oxides is known to occur, due to equilibrium with $\mathrm{Fe}(\mathrm{OH})_{x}{ }^{3-x}$ in the solution phase (Rich and Morel, 1990; Wells et. al., 1983). The rate of this process is 
related to the thermodynamic stability of the iron oxide phase; i.e. the thermal dissolution rate of a highly crystalline phase like goethite or hematite is much less than that of an amorphous phase like ferrihydrite. In general, the thermal dissolution rate of iron oxyhydroxides is slow relative to photoreductive dissolution.

Reactions in microenvironments - digestive vacuoles - The reduction of iron(III) in reducing microenvironments in oxic surface waters has been considered previously. Relevant microenvironments could include marine snow aggregates, diatom mats, fecal pellets, or even small organic colloids (Wells et. al., 1995). Reuter et. al. (1992) hypothesized that Trichodesmium colonies may utilize suboxic conditions in the colony interior to help solubilize iron oxide minerals.

Another reducing microenvironment worthy of consideration in this context are the digestive vacuoles of heterotrophic protists. As described in Chapter 1, vacuole chemistry is characterized by acidic $\mathrm{pH}$ (3-5), high levels of enzymatic activity, and high levels of reactive organic material. Reduction of iron(III) should certainly be favored under such conditions. Since protozoan grazers occur ubiquitously in the marine environment and consume $0.1-1 \mu \mathrm{m}$ size particles in the water column with turnover times on the order of days, they could potentially have a very significant impact on iron speciation by converting refractory particulate and colloidal forms of iron into more reactive, bioavailable phases. This chapter presents data documenting the dissolution of colloidal iron oxides in oxic seawater within the food vacuoles of nanoplanktonic protozoan grazers. The following section will present an overview of our experimental approach.

\section{Experimental approach}

Previous studies of the photochemical reduction of iron(III) in seawater (eg. Waite and Morel, 1984; Wells and Mayer, 1991a) and studies of the bioavailability of colloidal iron solids (Rich and Morel, 1990) have employed synthetic iron oxides. The use of these synthetic inorganic phases offers two important advantages: 1) Iron oxides occur very commonly in nature as the primary mineral phase of iron, one of the most abundant 
metals in the earth's crust. 2) Because of the ubiquity and importance of iron oxides in a variety of scientific disciplines, the structure, properties, and reactivity of various iron oxide phases have been extensively characterized (eg. Cornell and Schwertmann, 1996). Well-documented procedures for the laboratory synthesis of most of the known iron oxide minerals are widely available (eg. Schwertmann and Cornell, 1991).

In seeking to demonstrate the effect of protozoan grazers on iron speciation, therefore, synthetic iron oxides were used as a model phase for relatively refractory iron solids in seawater. In addition to the two advantages listed above, this approach has the advantage of being consistent with previous studies of iron chemistry in seawater, thereby facilitating evaluation of the current study in an established context. The experimental approach followed in this chapter is similar to that employed in Chapter 2, in that we have conducted a series of experiments in simplified model systems containing protozoan grazers and bacteria, with the added element of iron colloids. The synthesis protocols for and characteristics of this oxide phase are described in the first part of the following experimental section.

The experiments described in the experimental section are grouped into three subsets, based on the analytical techniques used to determine changes in the colloidal iron oxides as a result of protozoan grazing. Four independent methods were used to assess the effects of protozoan grazing on the colloidal iron. In the first set of experiments, techniques based on size fractionation and oxine lability were employed. These experiments were chronologically the first in the series. In the next set of experiments, a competitive ligand exchange/cathodic stripping voltammetry method (CLE/CSV) was used to assay for changes in the reactivity of the colloidal iron as a result of grazing. In the final set of experiments, iron-limited diatoms were employed as a "probe" to assay for the production of bioavailable iron from the colloids as a result of protozoan grazing. As in Chapter 2, results and discussion will be presented separately for each set of experiments in the experimental section, to be followed by a more general summary and discussion in the conclusion. 


\section{Experimental}

\section{Colloid Synthesis}

The colloids used in these experiments were synthesized according to the protocol of Wells et. al., 1991b. Ferric chloride $\left(\mathrm{FeCl}_{3} \cdot 6 \mathrm{H}_{2} \mathrm{O}\right)$ was added to Milli-Q water at room temperature to result in a final Fe concentration of $4 \times 10^{-4} \mathrm{M}$. This solution was allowed to polymerize at room temperature for 30-45 minutes. The $\mathrm{pH}$ in our system during this step was generally 3.2-3.4. For radiolabeled colloids, ${ }^{59} \mathrm{Fe}$ (as Fe(III)) was added to the solution just prior to dissolving the ferric chloride. At the end of the polymerization step, small aliquots of the colloidal sol $(\sim 20 \mathrm{ml})$ were heated for 5 minutes in a water bath. Most of the colloids used in these experiments were heated at $50^{\circ} \mathrm{C}$. In a few experiments, colloids heated at $90^{\circ} \mathrm{C}$ were used. After heating, colloids were rapidly cooled to room temperature by immersion in an ice water bath for about 5 minutes.

Following synthesis as described above, the colloidal sol was briefly extracted (15 minutes) in a slurry of Biorad 100-200 mesh AG50W-X8 cation exchange resin $\left(\mathrm{H}^{+}\right.$ form) at a pH of about 3 . This extraction was performed in order to remove any dissolved or highly reactive iron from the colloidal sol. To make the slurry, resin and colloidal sol were combined in a $1 / 20$ weight ratio (i.e. $1 \mathrm{~g}$ resin for every $20 \mathrm{~g}$ colloidal sol). After resin extraction, the colloidal sol was separated from the resin beads by filtration through a coarse glass frit (which retained the resin beads, but not the much smaller suspended colloids). For $50^{\circ} \mathrm{C}$ colloids, this procedure normally removed $40-50 \%$ of the $\mathrm{Fe}$, measured as ${ }^{59} \mathrm{Fe}$ before and after extraction. For $90^{\circ} \mathrm{C}$ colloids, only $10-20 \%$ of the ${ }^{59} \mathrm{Fe}$ was removed. For experiments, the colloids were added to seawater to give a final Fe concentration of about $1 \mu \mathrm{M}$.

The colloids formed by these synthesis and extraction protocols are ferrihydrite, similar to the $50^{\circ} \mathrm{C}$ FERR and $90^{\circ} \mathrm{C}$ FERR described by Wells et. al., (1983) and Wells et. al., (1991b). Because of the extra resin extraction step in our procedure, there is much less reactive iron (referred to in Wells et. al., (1991b) as 'primary phase') associated with our colloids than with the ones used by Wells et. al. Ferrihydrite synthesized in this way forms by a process of hydrolysis and polymerization at room temperature, followed by 
nucleation into submicron crystallites during the brief heating step. The reactive iron referred to as primary phase by Wells et. al., which is stripped away in our procedure by extraction with cation exchange resin, likely corresponds to monomeric $\mathrm{Fe}$ and polymers not yet deposited onto the surfaces of the nuclei which form during heating ( $\mathrm{Gu}$ and $\mathrm{Hsu}$, 1987; Schwertmann and Cornell, 1991). As the heat increases during the heating step, more of the $\mathrm{Fe}$ is able to nucleate, thus eliminating the reactive, or primary phase. This was noted by Wells et. al., (1991b). Our colloids correspond to the nucleated, less reactive 'secondary' phase, which is most likely similar in reactivity to the $90^{\circ} \mathrm{FERR}$ described by Wells et. al. (1991b).

This colloidal phase is most similar to the 6-line ferrihydrite described in various literature (for example, see Schwertmann and Cornell, 1991). Analysis of a concentrate of our colloidal sol by X-ray powder diffraction did not detect any crystallinity, consistent with Wells et. al., (1991b). This phase is more ordered than the very amorphous 2-line ferrihydrite, but still sub-crystalline. Figure 3.1 shows scanning electron micrographs of the concentrated colloidal sol, made by settling the colloidal suspension onto a formvar grid. No stains were used in these preparations. The SEM micrographs reveal a structure of filamentous aggregates ( $150 \mathrm{~nm}$ in length) of roughly spherical subunits (5-10 $\mathrm{nm}$ in size). The presence of these discrete subunits, and their size, corresponds to the morphology expected of a 6-line ferrihydrite. 2-line ferrihydrites are aggregated to the extent that subunits, which are only about $2 \mathrm{~nm}$ in size, are difficult to observe (Cornell and Schwertmann, 1996).

Although the colloid synthesis protocol is fairly reproducible, small variations in factors such as temperature, $\mathrm{pH}$, and concentration during synthesis can affect the level of reactivity of the colloidal ferrihydrite. The ferrihydrite also ages relatively quickly, undergoing reorganization into more stable forms in time periods on the order of days, even at room temperature (Wells et. al., 1991b). For these reasons, colloidal ferrihydrite was synthesized fresh for each experiment, and reactivity in experimental systems is always normalized to reactivity in controls run simultaneously. 

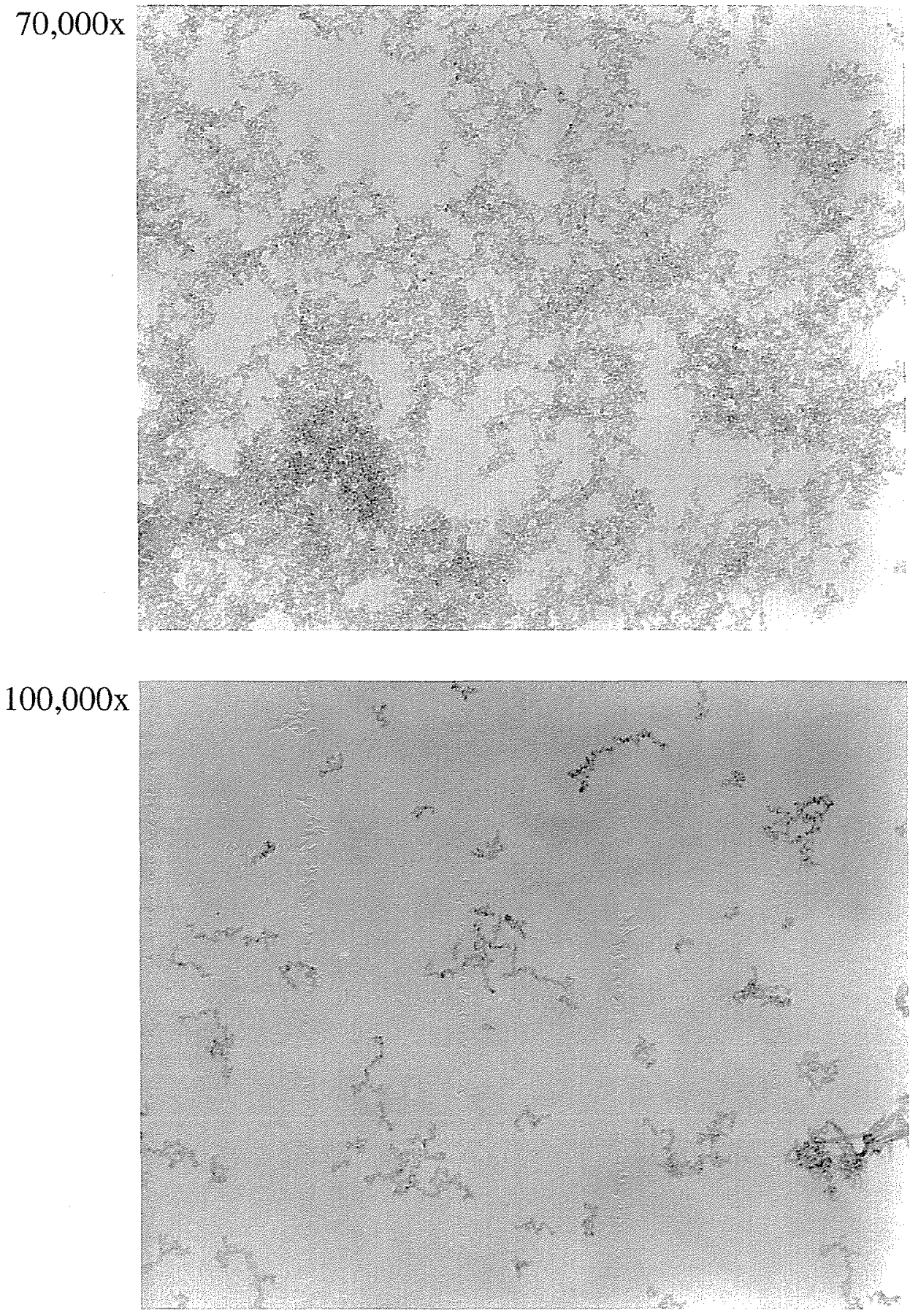

Figure 3.1- Scanning electron micrographs of concentrated colloidal ferrihydrite, at 70,000x (top) and 100,000x (bottom) magnification. These images were prepared by Barbara Eaglesham at Cornell University. 
Size Fractionation/Oxine Lability Experiments with ${ }^{59} \mathrm{Fe}$-labeled colloids

Methods - $0.05 \mu \mathrm{m}$ pore-size Nuclepore polycarbonate filters were used in size fractionation studies with radiolabeled colloids. Preliminary investigation demonstrated that colloids added to $0.2 \mu \mathrm{m}$ filtered, sterile Vineyard Sound water (VSW) at $\mu \mathrm{M}$ concentrations were $90-100 \%$ retained by $0.05 \mu \mathrm{m}$ filters. Since the ferrihydrite subunits, as described above, are only $0.005-0.01 \mu \mathrm{m}$ in size, their retention by $0.05 \mu \mathrm{m}$ filters must be due to aggregation, either amongst the iron colloidal subunits themselves, or with colloidal material naturally occuring in VSW. Thus this pore size was used as a cutoff to assay for disaggregation/dissolution of colloidal Fe due to protozoan grazing activity. Specifics of size fractionation protocols are discussed for the experiments below.

Oxine lability measurements were made according to Wells et. al., 1991b. These measurements were used to assay for grazer-mediated changes in colloid reactivity as determined by competitive ligand exchange with oxine. Wells et. al. have demonstrated a correlation between oxine exchangeable iron and biologically available iron in ferrihydrite colloids. To make the measurement, $20 \mathrm{ml}$ aliquots of whole-culture water were buffered to $\mathrm{pH} 6$ with phosphate buffer, and oxine was added to give a final concentration of $1.25 \times 10^{-4} \mathrm{M}$. After incubating for 1 hour at room temperature under these conditions, samples were filtered through a $0.2 \mu \mathrm{m}$ Nuclepore polycarbonate filter, and the filtrate was collected and passed through a C-18 Sep-Pak cartridge previously rinsed with methanol and Milli-Q water. Oxine-complexed ${ }^{59} \mathrm{Fe}$ was eluted from the SepPaks with methanol as described by Wells et. al., and radio-isotope counts in the eluate were normalized to counts of total ${ }^{59} \mathrm{Fe}$ activity in whole culture aliquots taken at the same time as the oxine lability sample aliquots.

Preliminary experiment - Model system set-up for this experiment included two controls: 1) Fe colloids in VSW at $\mu \mathrm{M}$ concentrations (colloid control); 2) Fe colloids plus bacteria in VSW (bacteria control). The experimental model system included $\mathrm{Fe}$ colloids and bacteria, and was inoculated with the microflagellate grazer Cafeteria. There were no replicate cultures in this preliminary experiment. As in Chapter 2, the bacteria 
(Halomonas halodurans) were first grown up separately in yeast extract medium and then harvested, rinsed, and resuspended for the experiment. The colloids were added to the VSW in control and experimental bottles (at $\mu \mathrm{M}$ concentrations) at the same time as the resuspended bacteria. It was assumed that the colloids would be scavenged by the surface of bacteria cells, and thus be consumed by the protists along with the bacterial prey. Protozoan grazers were inoculated into the experimental culture at $t_{0}$, and size fractionation and oxine lability measurements were taken at intervals thereafter. Model systems (1.5 liter each) were maintained in 2.5 liter polycarbonate bottles at room temperature under normal laboratory lighting, with no agitation except prior to sampling. For size fractionation, filtration was perfomed by gentle vacuum filtration through $47 \mathrm{~mm}$ diameter, $0.05 \mu \mathrm{m}$ pore size Nuclepore membranes $\left(10 \mathrm{ml}\right.$ per filter). ${ }^{59} \mathrm{Fe}$ activity was determined on filters by gamma counting, and normalized to whole culture ${ }^{59} \mathrm{Fe}$ samples of identical geometry. Oxine lability measurements were performed as described previously, at $\mathrm{t}=31$ hours and $\mathrm{t}=61$ hours.

Preliminary work indicated that wall loss of colloidal Fe could be a problem in these systems. In addition, any colloidal iron dissolved by the action of the grazers could be rapidly scavenged back onto the solid phase due to the high concentrations of bacteria and colloidal iron particles in these systems. This would prevent any ${ }^{59} \mathrm{Fe}$ from showing up in the $<0.05 \mu \mathrm{m}$ size fraction, despite grazer-mediated dissolution. Because of these concerns, $10^{-4} \mathrm{M}$ EDTA was added to all experimental and control cultures to act as a "trap" for dissolved iron. This is similar to the use of EDTA by Hutchins and Bruland, 1994 in their laboratory work on the remineralization of cellular Fe by grazers. Although some dissolution of colloids due to the presence of elevated concentrations of EDTA might be expected, preliminary data indicated that this would not be a large effect. In seawater at $\mathrm{pH} 8$, the ferrihydrite should be near neutrally charged (Cornell and Schwertmann, 1996) so that EDTA would have little affinity for colloid surfaces.

Additional data obtained in this preliminary experiment included a Ti(III)-EDTA (Hudson and Morel, 1989) wash procedure performed on the bacteria control about 24 hours after the last experimental time point. This was done in order to ascertain whether 
any of the ${ }^{59} \mathrm{Fe}$ associated with the bacteria was intracellular, therby indicating some solubilization and intracellular absorption of the Fe colloids by the bacteria after extended incubation. The Ti wash procedure was performed using a $47 \mathrm{~mm}$ diameter, $0.2 \mu \mathrm{m}$ pore size Nuclepore filter to retain all of the bacteria in a $20 \mathrm{ml}$ aliquot. The activity of the Tiwashed filter was normalized to the activity of a similar $20 \mathrm{ml}$ aliquot filtered onto a $0.2 \mu \mathrm{m}$ Nuclepore filter and rinsed with VSW.

The results of the preliminary experiment are shown in Figure 3.2. It can be seen from the oxine lability data at $t=31$ and $t=61$ that the oxine-labile percentage of the colloidal ${ }^{59} \mathrm{Fe}$ in the grazing culture was significantly higher (by a factor of 5-6) than in either of the controls (Fig. 3.2A). In the size fractionation data for the colloid control, ${ }^{59} \mathrm{Fe}$ $<0.05 \mu \mathrm{m}$ averaged about $30 \%$ for the duration of the experiment (Fig. 3.2B). This is higher than the normal percentage for colloids at $\mu \mathrm{M}$ concentrations in plain VSW, and this effect was probably due to the presence of EDTA, acting either to solubilize the colloids to some degree or to prevent aggregation by changing colloidal surface properties. In both the grazing culture and the bacteria control, only about $10 \%$ of the ${ }^{59} \mathrm{Fe}$ was $<0.05 \mu \mathrm{m}$ in size at $\mathrm{t}_{0}$, probably due to aggregation of the colloids with the bacteria. This percentage increased in both bacteria controls and grazing cultures by $\mathrm{t}=$ 21 , due most likely to the influence of EDTA. In the bacteria control the $\%$ of ${ }^{59} \mathrm{Fe}<$ $0.05 \mu \mathrm{m}$ leveled off at about $30 \%$, similar to the colloid control, until the very end of the experiment when it went up somewhat. In the Cafeteria grazing culture, the $\%$ of ${ }^{59} \mathrm{Fe}$ $<0.05 \mu \mathrm{m}$ increased throughout the experiment, reaching a maximum of about $80 \%$. Wall losses of ${ }^{59} \mathrm{Fe}$ in this experiment averaged about 20\%, even with EDTA added to reduce wall loss. The percentages shown in Figure 3.2 are normalized to the total ${ }^{59} \mathrm{Fe}$ in the system at each time point.

These preliminary results were encouraging, in that the protozoan grazing culture showed signs of both colloidal dissolution/disaggregation and increasing oxine reactivity relative to the control cultures. The Ti-wash of the bacteria control indicated that, out of the ${ }^{59} \mathrm{Fe}$ associated with the $>0.2 \mu \mathrm{m}$ size class (about $60 \%$ of the total ${ }^{59} \mathrm{Fe}$ in the system), only $1.8 \%$ was not removed by the Ti-wash procedure. This indicates little to no 

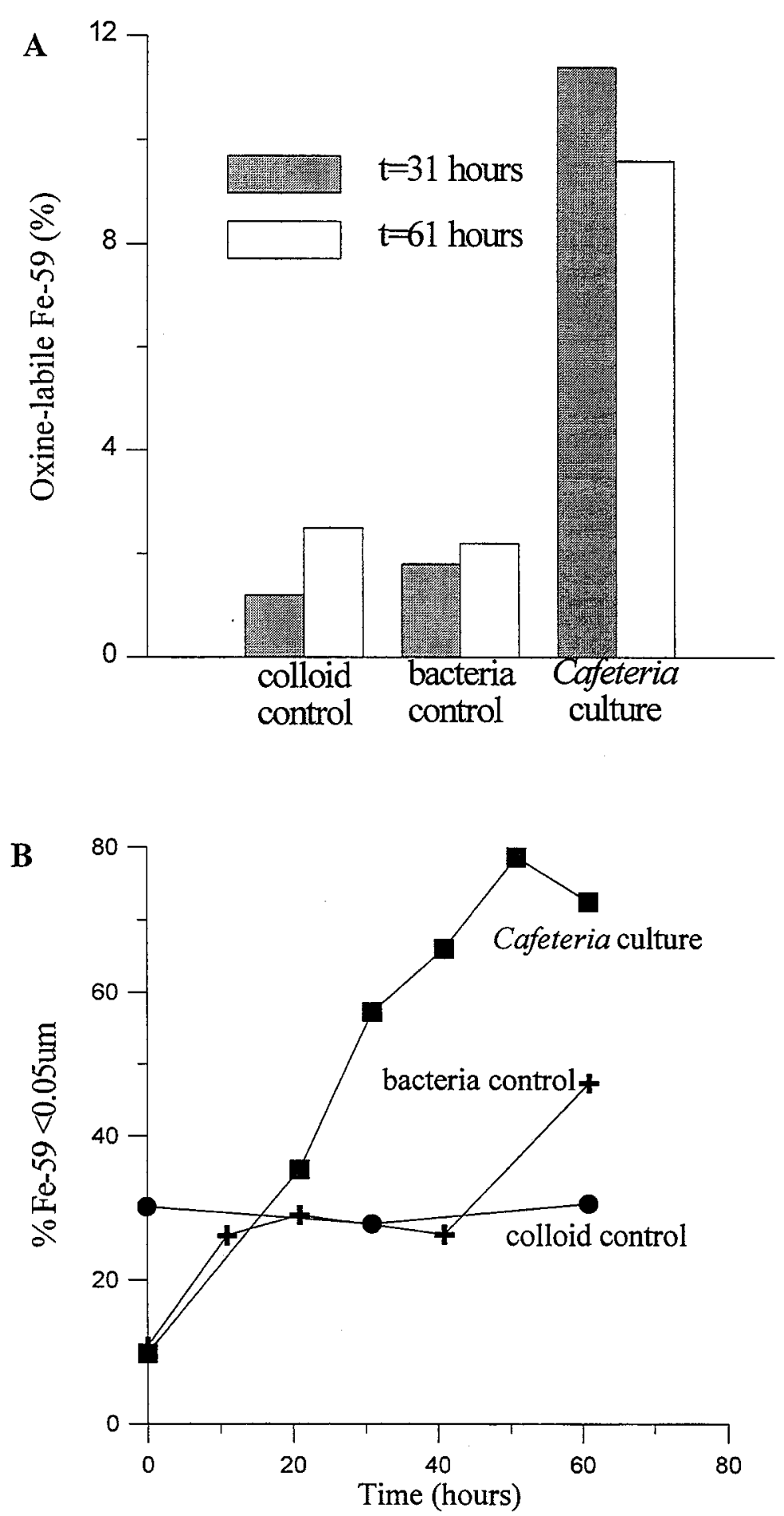

Figure 3.2 - Data from preliminary experiment in size fractionation/oxine lability series A. $\%$ oxine-labile iron in model systems at $t=31 \mathrm{hrs}$ and $\mathrm{t}=61 \mathrm{hrs}$.

B. \% Fe-59 less than $0.05 \mathrm{um}$ in size in different model systems vs. time 
solubilization and internalization of the iron by the bacteria, even after extended incubation. The bacteria in the system therefore appeared to have little effect on the colloidal Fe. A second experiment was thus planned involving replicate model systems and an additional grazer, Uronema. This experiment is described below.

Second experiment - The protocols followed in the next experiment were similar to those described for the preliminary experiment, with a few significant differences. Replicate model systems of colloid controls, bacteria controls, Cafeteria grazing cultures, and Uronema grazing cultures were maintained. (Culture volume 1 liter each, in 2.5 liter polycarbonate bottles). At $t_{0}$, the concentration of bacteria in bacteria control and grazing cultures was $8 \times 10^{7}$ cells $/ \mathrm{ml}$. The concentration of Cafeteria at $\mathrm{t}_{0}$ in grazing cultures was $7.3 \times 10^{3}$ cells $/ \mathrm{ml}$, and the Uronema concentration was $1.1 \times 10^{3}$ cells $/ \mathrm{ml}$. Colloids, as in the preliminary experiment, were added to all model systems at a concentration of about $1 \mu \mathrm{M}$. Also as in the preliminary experiment, all model systems contained $10^{-4} \mathrm{M}$ EDTA.

Instead of doing size fractionation by vacuum filtration, in-line filtration through $0.05 \mu \mathrm{m}$ Nuclepore polycarbonate filters was performed using a syringe filtration system. Filters were not allowed to dry during filtration. This method has been shown to be more gentle than vacuum filtration, leaving fragile protists intact (Nagata and Kirchman, 1990). Filtrates were collected to determine ${ }^{59} \mathrm{Fe}$ activity. Activity in filtrates was normalized to activity in geometrically identical aliquots of whole culture. Both filtrates and wholeculture aliquot samples were acidified to prevent wall loss (which could affect geometry). In-line filtration through a $0.2 \mu \mathrm{m}$ Nuclepore was also used in the oxine lability protocol, prior to running filtrates through C-18 Sep-Paks. Oxine lability samples were taken at all time points.

The oxine lability and size fractionation results for the second experiment are shown in Figure 3.3. As in the preliminary experiment, wall losses for this experiment averaged about $20 \%$, so percentages are normalized to the ${ }^{59} \mathrm{Fe}$ totals at each time point. The results for oxine lability were similar to those in the preliminary experiment. The Cafeteria cultures demonstrated a $\sim 10 \%$ increase in oxine lability relative to the controls, 

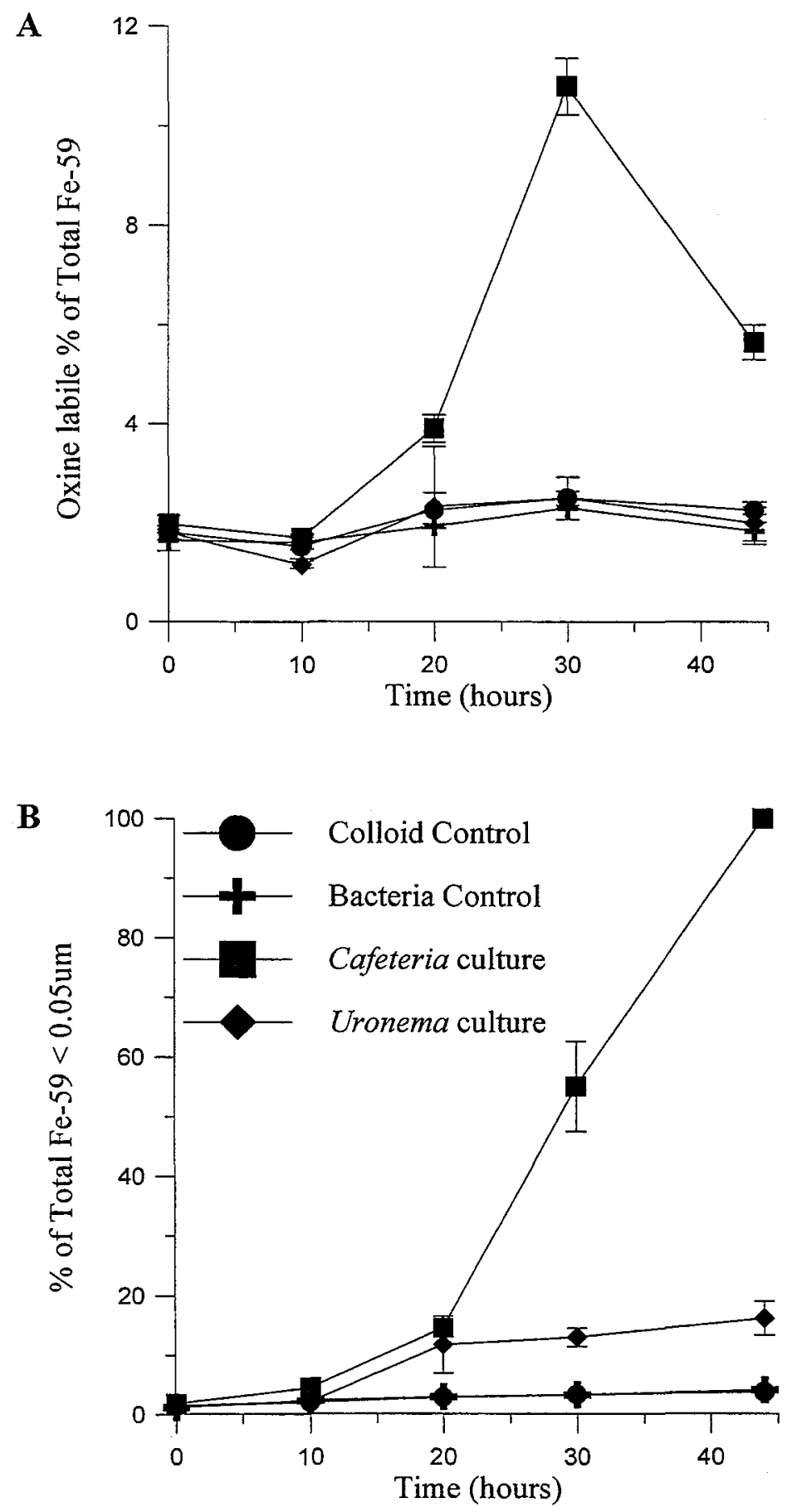

Figure 3.3 - Data from second experiment in size fractionation/oxine lability series. Symbol legend in B.

A. \% oxine-labile $\mathrm{Fe}-59$ vs time in different model systems.

B . $\% \mathrm{Fe}-59<0.05 \mathrm{um}$ vs time in different model systems 
with a peak at about 30 hours. This peak in oxine lability coincided with maximal rates of grazing in the Cafeteria cultures, as estimated by when decreases in the optical density of the grazing cultures relative to the control first became apparent. Oxine lability in the Uronema culture was not significantly different from the controls.

Size fractionation results demonstrated an increase of over $90 \%$ in ${ }^{59} \mathrm{Fe}<0.05 \mu \mathrm{m}$ in the Cafeteria cultures. A much smaller, but still significant increase $(\sim 10 \%)$, was shown in the Uronema cultures. Both control systems showed identical values of about 3-5\% for ${ }^{59} \mathrm{Fe}<0.05 \mu \mathrm{m}$ over the entire course of the experiment. This was significantly less than the control values for ${ }^{59} \mathrm{Fe}<0.05 \mu \mathrm{m}$ in the preliminary experiment, which were about $30 \%$. The difference was most likely due to differences in the reactivity of the iron colloids used in these experiments. The radiolabeled colloids synthesized for the second experiment differed somewhat from the normal protocol in that conditions during polymerization of the sol were (inadvertantly) more acidic than usual, causing slower polymerization. The lower reactivity of this batch of colloids was indicated by the results of the resin extraction step, which only removed about $20 \%$ of the ${ }^{59} \mathrm{Fe}$, vs $40-50 \%$ normally.

Most noticable in Fig. 3.3 is the difference in results for Cafeteria vs Uronema. Since these grazing cultures were run simultaneously, using the same batch of colloids and the same bacterial prey, the data appear to reflect interspecies differences in the effect of Uronema and Cafeteria on colloidal iron. Data taken on the $\mathrm{pH}$ of these cultures during the course of the experiment did not indicate any significant differences between the $\mathrm{pH}$ in the Cafeteria and Uronema cultures, so $\mathrm{pH}$ in the cultures cannot account for the observed differences in colloidal iron behavior (data not shown).

The fact that the peak in oxine lability in the Cafeteria grazing culture coincided with the period when the cultures first began to clear could indicate that oxine-reactive iron is an intermediate product, produced in greatest quantities at peak grazing rates and removed as grazing rates decline due to thermodynamic maturation of the colloidal iron phase. This effect was not observed in the size fractionation data, presumably because EDTA trapped the reactive iron produced by grazing in the dissolved phase. The question 
of how the presence of EDTA affects oxine lability is an interesting one, and this issue will be addressed in the next experimental section, where competitive ligand exchange with 1-nitroso-2-napthol is used to assess the role of EDTA in these systems.

\section{CLE/CSV with 1-nitroso-2-napthol}

Method - A further series of experiments was conducted using a competitive ligand exchange technique based on addition of the ligand 1-nitroso-2-napthol (1N2N) with detection by cathodic stripping voltammetry (CSV) (Wu and Luther, 1995). This technique is similar in terms of chemical principles to the oxine lability method. It also has the advantage that it can be used with CSV as the Fe detection method, allowing for relatively quick sample analysis without the necessity of using ${ }^{59} \mathrm{Fe}$ as a tracer for colloidal iron. Not having to use a radiotracer makes a number of aspects of the experimental work easier, allowing more experiments to be run in less time. Peter Croot is acknowledged for his work in adapting this technique for our purposes, and for his help in generating the data shown here.

The basic protocol for experimental set-up was similar to that described for the size fractionation/oxine methods described previously, with the exception of using radiolabeled iron colloids. Without ${ }^{59} \mathrm{Fe}$ as a tracer for colloidal iron, an additional control was necessary in order to account for production of labile iron due to consumption of bacterial biomass by the grazers. Thus, the basic set of controls included:

1) Colloidal ferrihydrite in VSW (colloid control); 2) Colloidal ferrihydrite plus $H$. halodurans in VSW (bacteria control); 3) H. halodurans in VSW inoculated with a protozoan grazer at $t_{0}$ (bacterial iron control). The experimental model systems, as in previously described experiments, included colloidal ferrihydrite, H. halodurans, and protozoan grazers inoculated at $t_{0}$. The experiments described in this section were carried out in square $250 \mathrm{ml}$ polycarbonate bottles, with $150 \mathrm{ml}$ of culture. Most model systems were run in replicate; exceptions are mentioned specifically below. Experiments were generally run over a time course of 48 hours, during which time bottles were incubated at 
room temperature without agitation except prior to sampling. 1N2N measurements were made at intervals (usually every 10-12 hours) during the course of an experiment.

For $1 \mathrm{~N} 2 \mathrm{~N}$ measurements, unfiltered $20 \mathrm{ml}$ aliquots were removed from each control and experimental system and placed in a separate teflon bottle. To these aliquots were added $200 \mu \mathrm{l}$ of PIPES buffer for a final $\mathrm{pH}$ of 6.9 , and $40 \mu \mathrm{l}$ of a $10 \mathrm{mM}$ stock of $1 \mathrm{~N} 2 \mathrm{~N}$ for a final $1 \mathrm{~N} 2 \mathrm{~N}$ concentration of $20 \mu \mathrm{M}$. Aliquots with these additions were incubated for 1 hour at room temperature prior to CSV analysis. (PIPES and 1N2N additions were made in a staggered fashion so that all samples could incubate for equal amounts of time prior to CSV analysis.) Samples were analyzed for iron on an EG\&G Princeton Applied Research Model 303A Static Mercury Drop Electrode coupled to an EG\&G Princeton Applied Research Model 264A Polarographic Analyzer/Stripping Voltammeter. Sampling conditions during CSV runs were as follows: 2 min purge; $60 \mathrm{~s}$ deposition (15 s if surfactants were a problem.) at $-0.2 \mathrm{~V}$; scan rate $10 \mathrm{mV} / \mathrm{s}$; pulse height $25 \mathrm{mV}$; final potential $-0.75 \mathrm{~V}$. As per $\mathrm{Wu}$ and Luther, 1995, all teflon incubation bottles, graduated cylinders, teflon sample cups and electrodes were preconditioned before use and in between sample runs in a solution of $1 \mathrm{~N} 2 \mathrm{~N}, \mathrm{Fe}(\mathrm{III})$ and PIPES in UV-irradiated Sargasso seawater. Bottles, etc. were only rinsed in Milli-Q water between uses so as to maintain conditioning. Prior to all sample runs, a run of Sargasso seawater was done to ensure that the electrode was conditioned and working properly, with no contamination. 1N2N-labile $\mathrm{Fe}$ was recorded as peak height in $\mathrm{nA}$, and converted to Fe concentrations in $\mathrm{nM}$ using the slope of standard additions made to the colloid control at each time point. Background Fe concentrations in VSW (total) were $\sim 21 \mathrm{nM}$.

Preliminary Experiment - Figure 3.4 shows the results of a preliminary experiment using $\mathrm{CLE} / \mathrm{CSV}$ with $1 \mathrm{~N} 2 \mathrm{~N}$ to look at changes in colloid lability as a result of grazing. Several species were compared for their ability to increase colloid reactivity, all grazing on the same batch of resuspended yeast-extract grown $H$. halodurans. Model systems run in this experiment included: one colloid control; two bacteria controls; one bacteria iron control for each protist species (i.e. 3 total); and two experimental bottles each for 


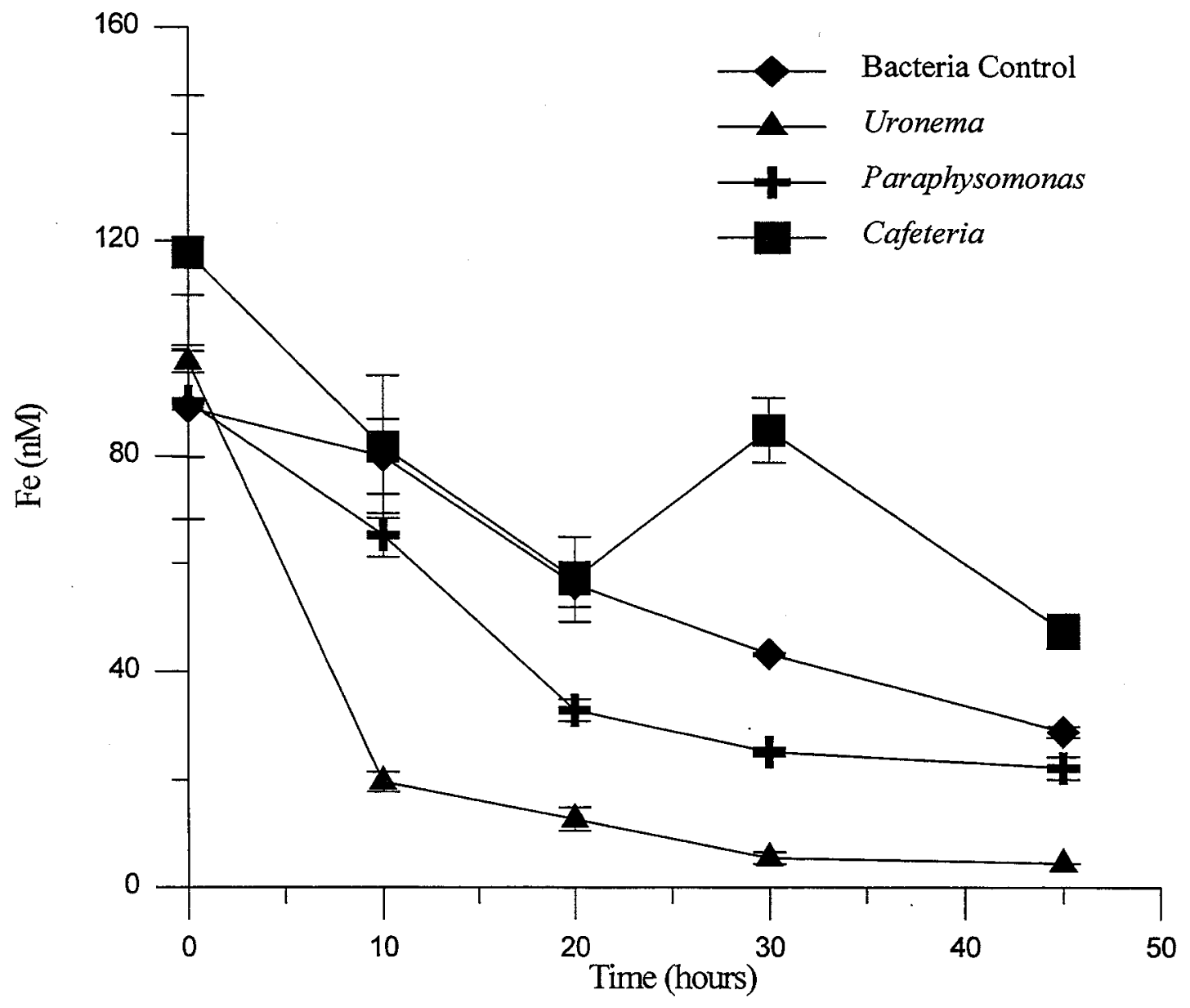

Figure 3.4 - Results of first experiment in CLE/CSV series. Multi-species screen test. $1 \mathrm{~N} 2 \mathrm{~N}$-labile $\mathrm{Fe}$ in different model systems vs time. 
Paraphysomonas, Cafeteria, and Uronema. Results are shown in Figure 3.4 only for the bacteria controls and the experimental cultures. While these results do appear to indicate some increase in the reactivity of the colloids in the Cafeteria culture, these data served mainly to demonstrate the potential of the method, and some of the possible pitfalls.

The initial values for reactive $\mathrm{Fe}$ at $\mathrm{t}_{0}$ were quite high. This problem was reduced in subsequent experiments by waiting about an hour between setting up model cultures and sampling for $t_{0}$. The high values were most likely due to some initial reactions when the colloids were added to the model system; reactive iron decreases relatively quickly as the system reaches equilibrium. These data also demonstrated the necessity of staggering sample additions so that each sample incubated only one hour with 1 N2N. Finally, this experiment revealed an unforseen difficulty in using this method: the cultures of the ciliate, Uronema, produced such copious amounts of surfactant material that measurements were not possible, even with deposition times reduced to 15 seconds instead of the usual 60 seconds. This problem was not encountered in either of the flagellate cultures, Cafeteria or Paraphysomonas. Later experimentation with other protozoan species showed this to be a consistent phenomenon: Ciliate grazers produced too much surfactant material for practical measurement via CLE/CSV, whereas flagellates did not.

Mechanistic Experiments - Modifications in protocol based on the results of the preliminary experiment described above resulted in improved ability to make CSV measurements in culture. This is demonstrated by the data in Figure 3.5A-C, in which $\mathrm{CLE} / \mathrm{CSV}$ was used to investigate a number of mechanistic details involving the role of EDTA in our model systems, the reactivity of ferrihydrite synthesized with a $50^{\circ} \mathrm{C}$ vs a $90^{\circ} \mathrm{C}$ heating step, and the effect of varying bacterial concentrations in grazing cultures on changes in colloid lability. The data shown in Figure 3.5 were all obtained in the same experiment, using the same batch of bacteria and the same two batches of colloidal ferrihydrite, $50^{\circ} \mathrm{C}$ and $90^{\circ} \mathrm{C}$; data is graphed on three separate plots in order to illustrate 

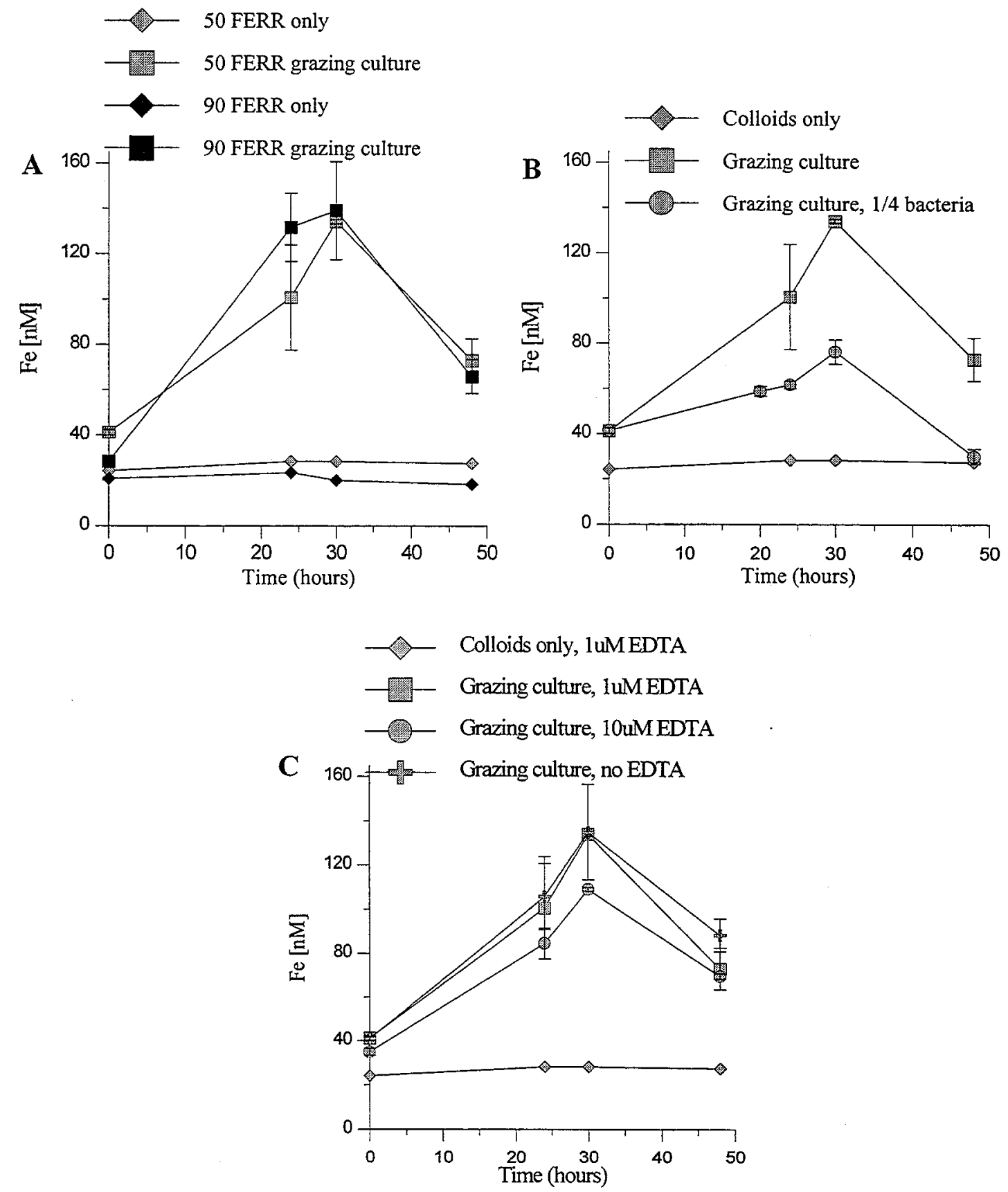

Figure 3.5 - Mechanistic experiments with CLE/CSV: 1N2N-labile Fe vs time A - Grazer effects on 50 FERR vs 90 FERR B - Effect of bacteria concentration on dissolution of colloidal iron oxides by grazers

C - Effect of EDTA concentration on dissolution of colloidal iron oxides by grazers 
specific points. Bacteria used in this experiment were resuspended yeast-extract grown $H$. halodurans. The following model systems were run, all in VSW as the medium:

$-50^{\circ} \mathrm{C}$ FERR + bacteria + Cafeteria; $1 \mu \mathrm{M}$ EDTA (replicates)

$-50^{\circ} \mathrm{C}$ FERR + bacteria + Cafeteria; $10 \mu \mathrm{M}$ EDTA (replicates)

- 50 ${ }^{\circ} \mathrm{C} \mathrm{FERR} \mathrm{+} \mathrm{bacteria} \mathrm{+} \mathrm{Cafeteria;} \mathrm{no} \mathrm{EDTA} \mathrm{(replicates)}$

$-90^{\circ} \mathrm{C}$ FERR + bacteria + Cafeteria; $1 \mu \mathrm{M}$ EDTA (replicates)

$-50^{\circ} \mathrm{C} \mathrm{FERR}+1 / 4$ bacteria + Cafeteria $; 1 \mu \mathrm{M}$ EDTA (replicates)

- $50^{\circ} \mathrm{C}$ FERR; $1 \mu \mathrm{M}$ EDTA (1 bottle)

- $90^{\circ} \mathrm{C}$ FERR; $1 \mu \mathrm{M}$ EDTA (1 bottle)

Figure $3.5 \mathrm{~A}$ indicates that there was little difference in the reactivity of $50^{\circ} \mathrm{C} \mathrm{FERR} \mathrm{vs}$ $90^{\circ} \mathrm{C} \mathrm{FERR}$, either at a base level with respect to $1 \mathrm{~N} 2 \mathrm{~N}$ reactivity, or in response to protozoan grazing. In terms of the actual percentage of the colloidal iron that was reactive, the difference between the two would increase due to the fact that the 15 minute cation resin extraction step removed more of the $50^{\circ} \mathrm{C}$ FERR than the $90^{\circ} \mathrm{C}$ FERR (see earlier discussion of extraction protocol). Based on the usual variance between the two in this extraction step, if the values in Fig. 3.5A were graphed on a percentage basis, reactive iron for $50^{\circ} \mathrm{C}$. FERR would exceed that for $90^{\circ} \mathrm{C}$ FERR by about a factor of 1.5 . Thus, the $50^{\circ} \mathrm{C}$ FERR was actually more reactive. This may be due to surface area - the subunits in the $50^{\circ} \mathrm{C}$ FERR are likely to be smaller than those in the $90^{\circ} \mathrm{C} \mathrm{FERR,} \mathrm{(with}$ greater surface area to volume ratio), so the greater amount of exposed surface area could account for increased \% solubilization.

When only $1 / 4$ as much bacteria were added to the model system, the peak value of reactive iron decreased by about a factor of two (Fig. 3.5B). This is generally consistent with the idea that the production of reactive iron in the system is related to the ingestion of the Fe colloids by protists: The relationship between protozoan ingestion rate and particle concentration is nonlinear, due to saturation of the ingestion rate at higher particle concentrations (Fenchel, 1987). Assuming, then, that colloidal Fe ingestion rate in these systems is proportional to bacterial ingestion rate (given that the Fe colloids are most likely associated with bacterial surfaces), and further that production of reactive 
iron is proportional to colloidal ingestion rate, then a four-fold decrease in bacteria concentration should result in a less-than-four-fold decrease in production of reactive iron. This agrees with the result in Figure 3.5B.

There was no apparent difference in the peak value of reactive iron between the grazing culture with $1 \mu \mathrm{M}$ EDTA vs the grazing culture with no EDTA (Fig. 3.5C). Thus the EDTA itself did not play a role in increasing the amount of reactive iron in the system. In fact, as figure $3.5 \mathrm{C}$ shows, when the amount of EDTA in the system was increased from $1 \mu \mathrm{M}$ to $10 \mu \mathrm{M}$, there was actually a drop in the peak value of $1 \mathrm{~N} 2 \mathrm{~N}$ reactive iron. This was most likely due to competition for reactive iron between the EDTA (at $10 \mu \mathrm{M}$ concentration) and $1 \mathrm{~N} 2 \mathrm{~N}$ (at $20 \mu \mathrm{M}$ concentration). Because EDTA at elevated concentrations was able to compete with $1 \mathrm{~N} 2 \mathrm{~N}$, the Fe-1N2N CSV signal was drawn down.

Given this interpretation of the data in Figure 3.5C, how is it that any increase in reactive iron was detected at all in the previous experimental section, using the oxine lability method in systems with 10 times as much $(100 \mu \mathrm{M})$ EDTA? The following conceptual model is proposed to explain the competitive ligand exchange data in this chapter obtained with $1 \mathrm{~N} 2 \mathrm{~N}$ and oxine, in systems with and without EDTA: In the oxine lability experiments, oxine was added at a concentration of $125 \mu \mathrm{M}$, so it was still in excess of the EDTA present. Oxine is a much stronger chelator for iron than $1 \mathrm{~N} 2 \mathrm{~N}$. The equilibrium constant for $\mathrm{Fe}$ (oxine) $)_{3}$ in seawater is about $10^{38}$ (Wells et. al., 1991; Smith and Martell, 1975), while that for $\mathrm{Fe}(1 \mathrm{~N} 2 \mathrm{~N})_{3}$ is about $10^{28}$ (Wu and Luther, 1995). Thus, unlike $1 \mathrm{~N} 2 \mathrm{~N}$, oxine should be able to outcompete EDTA for reactive iron, even when it is only in excess of EDTA by a factor of 1.25 . The reactive iron bound by oxine is labile iron, produced from the colloidal ferrihydrite by the grazing process, which has not yet been bound by EDTA. Competition between $\mathrm{Fe}^{3+}$ and $\mathrm{Ca}^{2+} \mathrm{Mg}^{2+}$ for EDTA significantly slows down the kinetics of the Fe-EDTA reaction (Morel, 1983; Hering and Morel, 1988). Unlike EDTA, oxine has very little affinity for divalent alkali earth cations in seawater (Wells et. al., 1991b), so it should be able to rapidly bind reactive iron present in the system at any given time. 
It is probable that oxine is not, however, within a 1 hour incubation time, able to recover iron that has already been bound by EDTA (see Wu and Luther, 1995 for related kinetic studies on Fe-EDTA and $1 \mathrm{~N} 2 \mathrm{~N}$ ). This is the most likely explanation for the data in Figures 3.2 and 3.3. The ${ }^{59} \mathrm{Fe}$ less than $0.05 \mu \mathrm{m}$ in size in the Cafeteria grazing cultures corresponds to ${ }^{59} \mathrm{Fe}$ bound by EDTA, and this fraction of the ${ }^{59} \mathrm{Fe}$ increased throughout the grazing experiment. The data on oxine lability only showed detectable increases in labile iron during those time periods when the production of reactive iron by the grazing process outpaced the fairly slow kinetics of Fe-EDTA complexation, thereby leaving a pool of reactive iron detectable by the kinetically fast oxine lability method. This should explain why the oxine lability data had a peak at about 30 hours, the most intense grazing period. The same reasoning can be applied to the $1 \mathrm{~N} 2 \mathrm{~N}$ data in Figure 3.5.

As Figure 3.5C shows, competitive ligand exchange techniques reveal a peak in the temporal distribution of reactive iron in grazing cultures regardless of the presence of EDTA. This is because the reactive iron produced through grazing can also become unavailable to complexation by $1 \mathrm{~N} 2 \mathrm{~N}$ (or oxine) by means of hydrolysis reactions, precipitation, and thermal maturation of precipitated products (Wells et. al., 1991). When present, EDTA probably increases the rate of removal of iron from the reactive pool, as is shown by the 48 hour time point in Figure 3.5C: The amount of reactive iron remaining in the system with no EDTA was significantly higher than in the systems with EDTA.

Glucose-grown bacteria experiment - The data shown in Figure 3.4 for the preliminary experiment raised some concern about the possibility that the CSV baseline signal could be elevated due to chemistry associated with the bacteria in the system. In an attempt to bring the baseline down somewhat, an experiment was performed in which the bacterial prey, H. halodurans, was grown up on glucose medium (VSW with $1560 \mathrm{mg} / \mathrm{l}$ glucose, $240 \mathrm{mg} / 1 \mathrm{NH} / \mathrm{Cl}$ and $37 \mathrm{mg} / 1 \mathrm{NaH}_{2} \mathrm{PO}_{4}$ added) instead of on yeast extract medium as normally done for experiments. It was surmised that bacteria grown on glucose might provide a cleaner matrix for the model systems than bacteria grown on yeast extract. The glucose-grown bacteria were harvested and rinsed with VSW by centrifugation in the 
same way as normally done for yeast-extract grown cells. The experiment was conducted using Cafeteria as the grazer, inoculated from a culture growing on resuspended glucosegrown bacteria. No EDTA was added to the model systems in this experiment. Model systems included 2 colloid controls, 2 bacteria controls, 2 bacterial iron controls, and 2 experimental cultures.

The results of the experiment using glucose-grown bacteria are shown in Figure 3.6. Errors are the standard deviation of replicates. Using bacteria grown on glucose did reduce the background signal in the controls, and a clear peak in reactive iron was observed in the experimental cultures containing protists and $\mathrm{Fe}$ colloids, right around 30 hours as observed previously with oxine. The left $y$-axis expresses the concentration of $1 \mathrm{~N} 2 \mathrm{~N}$-labile iron in the samples as determined by standard additions. In the right $y$-axis, the concentration of $1 \mathrm{~N} 2 \mathrm{~N}$-labile iron has been normalized to the concentration of added colloidal iron $(\sim 1 \mu \mathrm{M})$. The right axis is thus directly comparable to the $y$-axis in the oxine lability graph in Figure 3.3 for a previous experiment with Cafeteria. It can be seen that the data compares remarkably well.

Figure 3.6 also seems to indicate that there was some increase in the reactivity of the Fe colloids in the bacteria control during the latter part of the experiment. This is supported by the data in the following figure, 3.7 , which shows the results of some extended $1 \mathrm{~N} 2 \mathrm{~N}$ incubation studies performed during the same experiment. At $\mathrm{t}=22$ hours and at $t=44$ hours, $1 \mathrm{~N} 2 \mathrm{~N}$ incubations of 4 and 8 hours in length were performed in addition to the usual 1 hour incubations. At $t=22$ hours, the $1 \mathrm{~N} 2 \mathrm{~N}$-labile $\mathrm{Fe}$ in the bacteria control was very similar to that in the colloid control. In contrast, the extended incubations at $\mathrm{t}=44$ show that the 8 -hour incubation with the bacteria control produced almost as much $1 \mathrm{~N} 2 \mathrm{~N}$-labile iron as the experimental culture.

This data raised concerns about the effect of the bacteria in these model systems on the the colloidal Fe. One possibility suggested was that the apparent release of reactive colloidal $\mathrm{Fe}$ as a result of grazing could actually be due to solubilization and absorption of colloidal iron by the bacteria, with subsequent release of iron from the bacteria as a result of protozoan grazing and excretion. The Ti-wash results discussed in the 


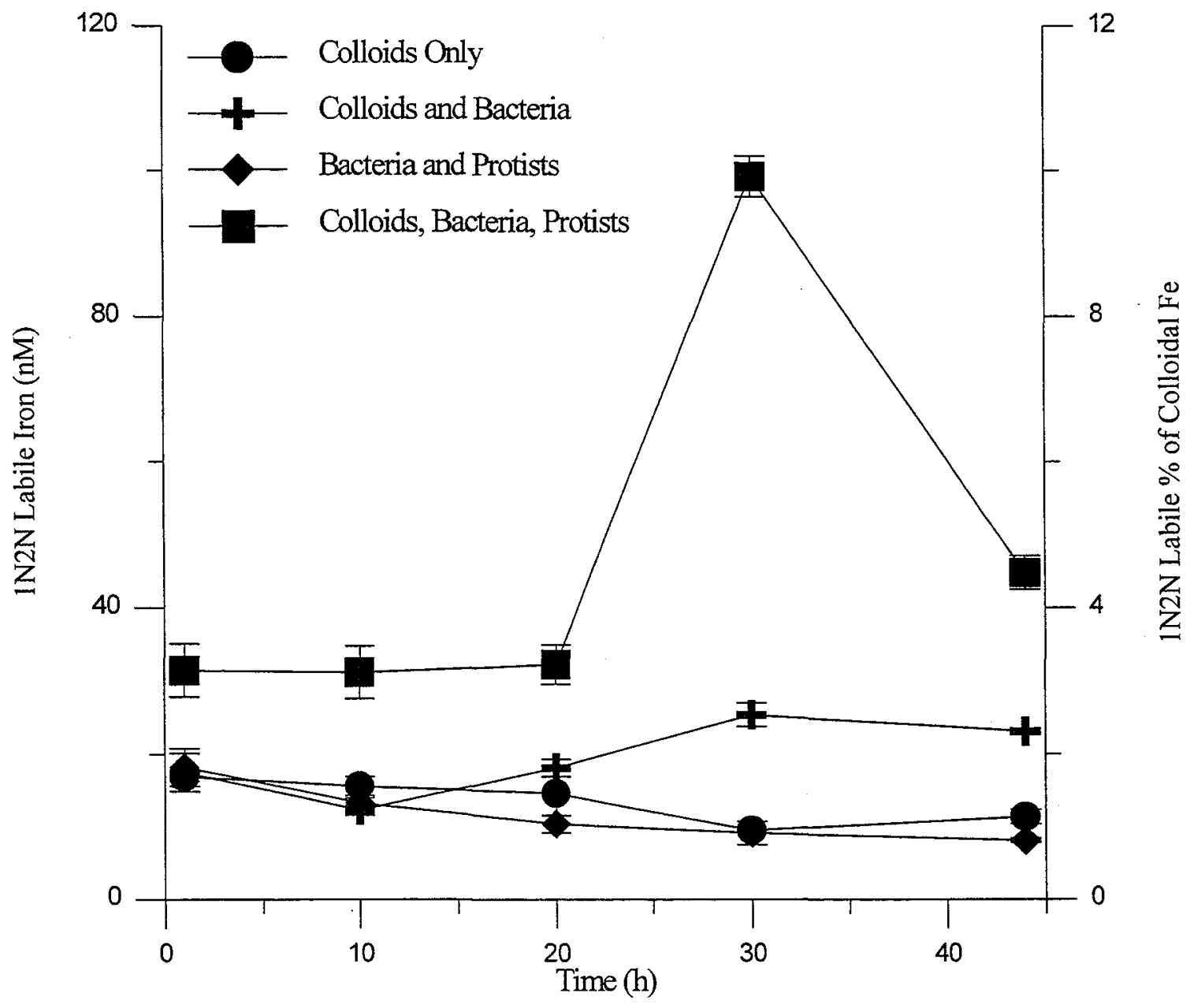

Figure 3.6 - Results from CLE/CSV experiment using glucose-grown bacterial prey to improve baseline. Cafeteria as grazer. Data shown are from 1 hour $1 \mathrm{~N} 2 \mathrm{~N}$ incubations. Left y-axis: $1 \mathrm{~N} 2 \mathrm{~N}$-labile Fe, in $\mathrm{nM}$. Right $\mathrm{y}$-axis: \% of colloidal $\mathrm{Fe}$ that is $1 \mathrm{~N} 2 \mathrm{~N}$ labile. This axis is comparable to $\mathrm{y}$-axis in Figure 3.3A. 

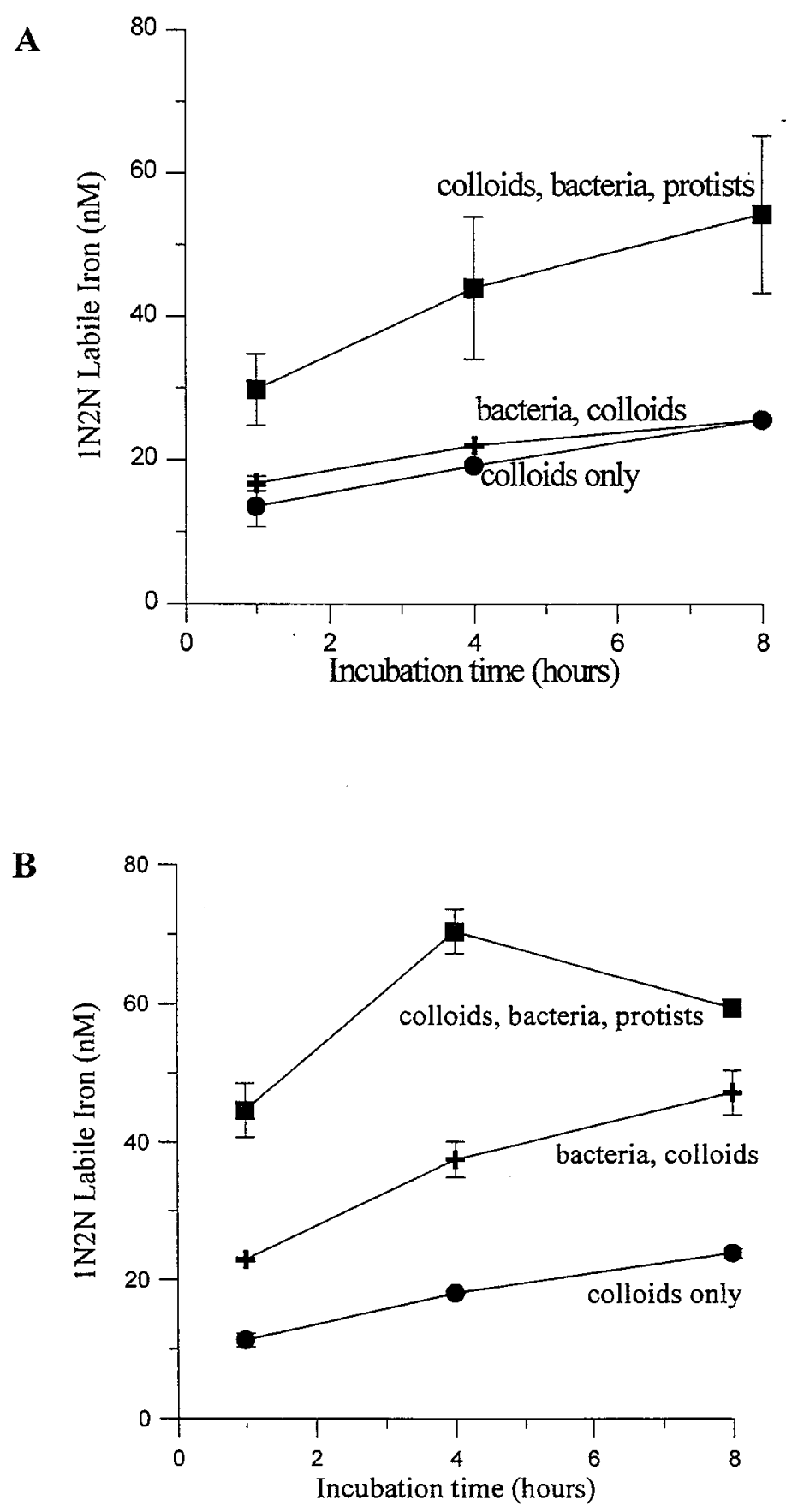

Figure 3.7 - Extended incubation data from CLE/CSV experiment with glucose-grown bacteria (see Fig. 3.6).

$1 \mathrm{~N} 2 \mathrm{~N}$-labile $\mathrm{Fe}$ (y-axis) vs $1 \mathrm{~N} 2 \mathrm{~N}$ incubation time (x-axis).

A. Data from 1-,4-,8-hour $1 \mathrm{~N} 2 \mathrm{~N}$ incubations at $\mathrm{t}=20 \mathrm{hrs}$ in experiment.

B. Data from $1-, 4-, 8$-hour $1 \mathrm{~N} 2 \mathrm{~N}$ incubations at $\mathrm{t}=44 \mathrm{hrs}$ in experiment. 
preliminary size fractionation/oxine lability experiment argue strongly against this, in that only $1.8 \%$ of the ${ }^{59} \mathrm{Fe}$ associated with the bacteria in the bacteria control for that experiment was intracellular, even after over 80 hours of incubation time. However, in order to fully discount the effects of bacteria, it was felt that an experiment using heatkilled bacteria would be necessary.

Heat-killed bacteria experiments - Conducting a CLE/CSV experiment with heat-killed bacteria proved to be unfeasible for several reasons. Attempts to conduct experiments with heat-killed glucose-grown bacteria failed because none of the protozoan strains in our collection would grow well, if at all, on heat-killed glucose-grown bacteria. The protists grew well on heat-killed yeast-extract-grown bacteria, but repeated attempts to conduct CLE/CSV experiments in model systems with heat-killed yeast-extract-grown bacteria failed because of an apparent matrix effect that caused interference with the CSV analysis. This was detected by comparison of the standard addition slopes for various model systems. The data for one of these comparisons is shown in Figure 3.8. For clearer comparison, all of the standard addition curves have been normalized to $0 \mathrm{nA}$ at 0 added [Fe]. As the figure shows, the standard addition slope in the heat-killed bacteria control was significantly low relative to other systems, indicating some kind of interference with the CSV signal. The slope in experimental systems was also somewhat erratic. The issue of conducting an experiment with heat-killed bacteria will be addressed in the final experimental section, in which $\mathrm{Fe}$-limited diatoms were used as probes for bioavailable Fe produced as a consequence of protozoan ingestion of colloidal ferrihydrite. In the last experiment in this section, CLE/CSV was used to determine whether a grazing culture from which the protists were removed by filtration could have the same effect on colloidal ferrihydrite reactivity as a grazing culture containing protists.

Grazing culture filtrate experiment - In order to substantiate our interpretation of the mechanistics of grazer-mediated effects on colloidal ferrihydrite as being due to processes occurring within grazer food vacuoles, an experiment was conducted in which 


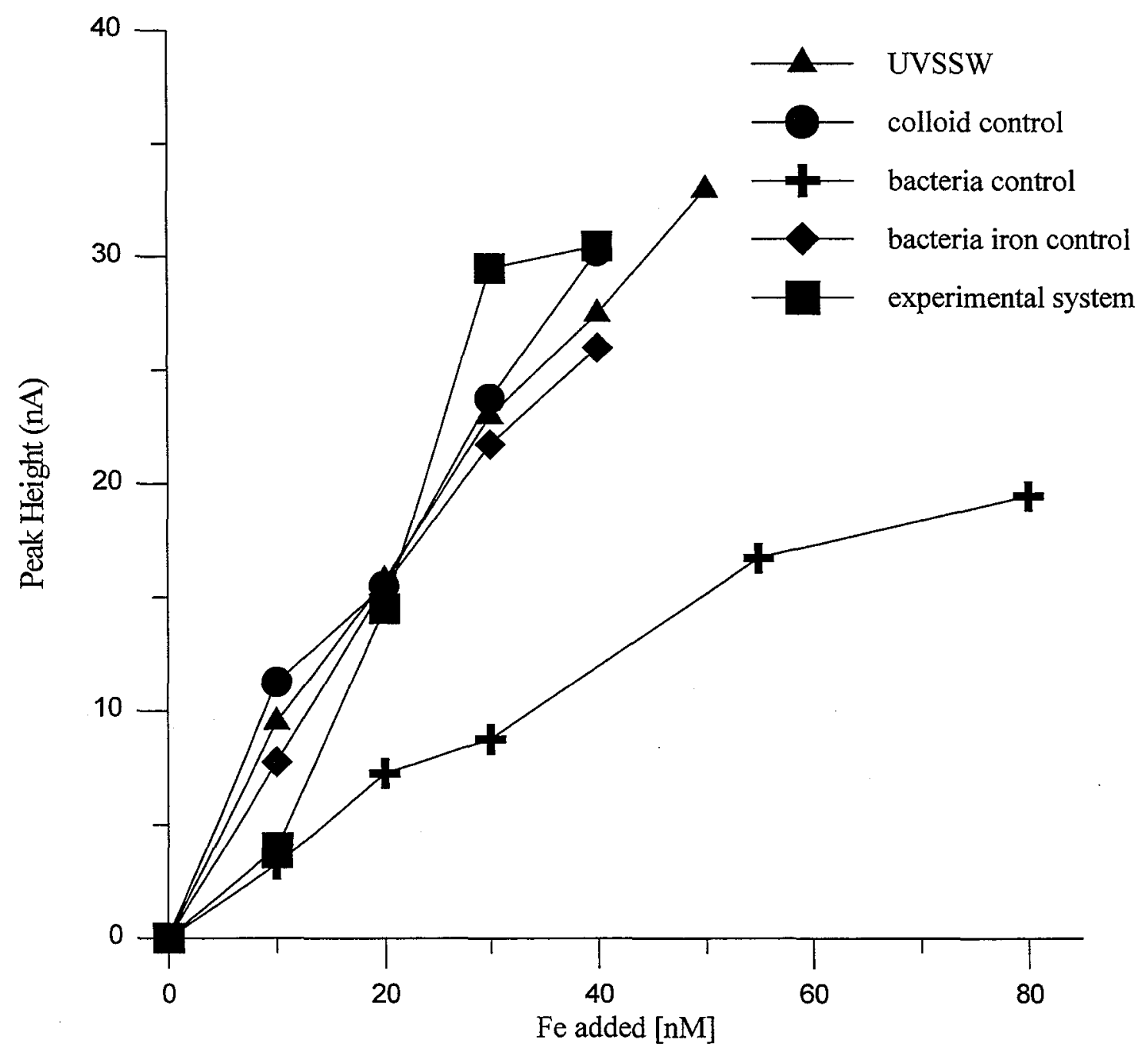

Figure 3.8 - Standard addition curves for CLE/CSV experiment with heat-killed, yeast-extract-grown bacteria as prey. Raw data shown as peak height in $\mathrm{nA}$ vs $\mathrm{Fe}$ additions in $\mathrm{nM}$. All curves normalized to $0 \mathrm{nA}$ at $0 \mathrm{Fe}$ additions. 
an actively grazing protozoan culture was filtered to remove protists, and the filtrate added to a suspension of colloidal ferrihydrite in VSW. CLE/CSV with $1 \mathrm{~N} 2 \mathrm{~N}$ was used to monitor changes in colloidal ferrihydrite reactivity as a result of the added culture filtrate. The purpose of this experiment was to demonstrate that the increase in colloidal ferrihydrite reactivity observed in previous experiments was not due to changes in the dissolved chemistry of the model system as a consequence of grazing activity. Protozoan grazers have been shown to release dissolved organic matter (Nagata and Kirchman, 1992), including extracellular enzymes that function at ambient seawater $\mathrm{pH}$ (Karner et. al., 1994). These kinds of processes could conceivably have an effect on Fe oxide reactivity.

For this experiment, the following model systems were set up in order to monitor changes in Fe colloid reactivity as a result of grazing: one colloid control, one bacteria control, one bacteria iron control, and one experimental culture containing Fe colloids, bacteria, and the grazer Cafeteria inoculated at $\mathrm{t}_{0}$. Starting at $\mathrm{t}_{0}$, CLE/CSV sampling was performed on these four model systems (referred to as monitoring cultures hereafter) in order to monitor changes in $\mathrm{Fe}$ colloid reactivity in the presence of the grazer. Four additional model systems were set up at the same time, but not monitored by CLE/CSV: replicate colloid controls, and replicate bacteria iron controls (just Cafeteria and bacteria). The same batch of colloidal ferrihydrite, bacterial prey, and protistan inoculum were used to set up all eight of these model systems. The H. halodurans used as prey in these systems were grown on glucose medium, and were not heat-killed.

As soon as the monitoring cultures indicated a substantial change in the reactivity of the Fe colloids in the presence of grazing Cafeteria, the replicate bacteria iron control cultures that had been set up at the same time as the monitoring cultures were gently filtered through a $47 \mathrm{~mm}$ diameter, $1 \mu \mathrm{m}$ pore size Nuclepore polycarbonate filter, using inline filtration driven very slowly by a peristaltic pump. This removed almost all of the protists and some of the bacteria. This was done when all of the model systems were 30.5 hours old, and the cultures containing grazers were just starting to clear. The replicate colloid control bottles were then split into four bottles, each half the volume of the 
original replicates. The $1 \mu \mathrm{m}$ grazing culture filtrate was added to two of these bottles, bringing them back to their original volume and thus diluting the colloidal $\mathrm{Fe}$ concentration by $50 \%$. VSW was added to the two remaining colloid control bottles, also to a level corresponding to a $50 \%$ dilution of colloidal Fe. These bottles served as controls for the colloid control/filtrate mixes. Aliquots were immediately removed from all four of these bottles for analysis by CLE/CSV with $1 \mathrm{~N} 2 \mathrm{~N}$. Time between filtering grazing cultures and adding $1 \mathrm{~N} 2 \mathrm{~N}$ reagents to colloid control/filtrate mixes was no more than 10-15 minutes. As soon as the $1 \mathrm{~N} 2 \mathrm{~N}$ incubation bottles were freed up from this $\mathrm{t}_{0}$ analysis, another set of aliquots was taken. CLE/CSV analysis of both the colloid control/VSW and colloid control/filtrate mixes was performed at intervals over the next 36 hours. Additional analyses were also performed on the monitoring cultures over the same time period.

For intercomparison, the data for this experiment (Fig. 3.9) is presented as peak height in $\mathrm{nA}$, with both $y$-axes on the same scale. There are no errors for the monitoring cultures, (Fig. 3.9 A) since there were no replicates of these cultures. The errors for the filtrate mixes (Fig. 3.9 B) are the standard deviation of replicates. The monitoring cultures demonstrated a large increase in colloidal Fe reactivity at 30.5 hours, when the replicate bacteria+Cafeteria cultures were filtered and the colloid+VSW and colloid+filtrate mixes were set up. This peak in the monitoring cultures persisted until about 40 hours, and then began to fall off. Reactivity in the bacteria control did appear to be consistently higher than in the colloid control.

Both the colloid+VSW and colloid+filtrate mixes exhibited a maximum in reactivity just after set-up (Fig. 3.9 B). The reactivity did appear to be higher in the colloid+filtrate than in the colloid+VSW. Reactivity rapidly dropped off in both of the colloidal suspensions by the next data point. There was some increase in reactivity in the colloid+filtrate mix at $t=40$. This may have been due to the residual presence of a few protozoan grazers in these bottles, confirmed by microscopic inspection. Due to the observed presence of protists in the colloid+filtrate mixes, at this time point all four mix bottles were moved into a lower temperature room for incubation (about $17^{\circ} \mathrm{C}$ as opposed 

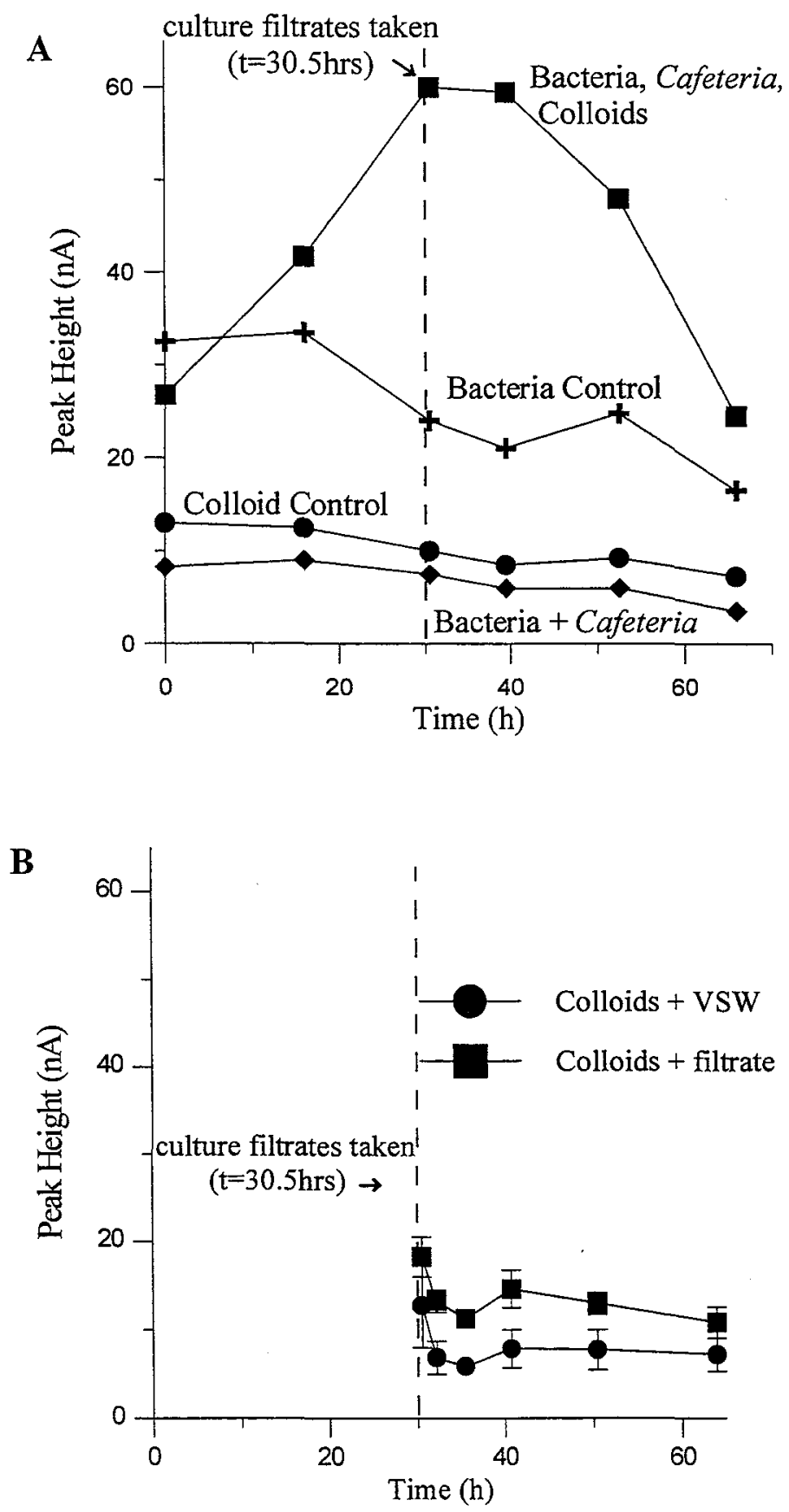

Figure 3.9 - CLE/CSV data from grazing culture filtrate experiment. Raw data shown as peak height vs time for comparison.

A. $1 \mathrm{~N} 2 \mathrm{~N}$-labile $\mathrm{Fe}$ in monitoring cultures. No replicates.

B. $1 \mathrm{~N} 2 \mathrm{~N}$-labile $\mathrm{Fe}$ in culture filtrates vs. VSW. Errors=std. dev. $(\mathrm{n}=2)$.

Dotted line marks when culture filtrates were mixed with colloid controls. 
to $24^{\circ} \mathrm{C}$ ) in order to reduce protozoan growth rate to some extent. Both colloid+filtrate and colloid+VSW mixtures leveled off to low reactivities by $t=66$.

Considering that the colloid+filtrate mixes were half as concentrated as the experimental monitoring culture, one might expect peak heights of about $30 \mathrm{nA}$ in the colloid+filtrate mixes had the Fe colloids therein reacted to the same extent as those in the experimental monitoring culture. Instead, after the initial time point, the colloid+filtrate mixes quickly equilibrated to a reactivity level of about 10-15nA, more commensurate with the reactivity level in the bacteria control of the monitoring cultures, which averaged about $25 \mathrm{nA}$. This is consistent with the composition of the culture filtrate, which was mostly bacteria. Thus the culture filtrate alone did not generate the same type of effect on colloidal ferrihydrite as a culture containing protozoan grazers. This supports the theory that protozoan ingestion of colloidal Fe, rather than dissolved or colloidal material released into the medium as a consequence of grazing, was responsible for the grazer-mediated effects on colloidal Fe reactivity observed in this and other experiments in this chapter.

This is also consistent with the kinetics observed for the oxine- and $1 \mathrm{~N} 2 \mathrm{~N}$-reactive iron analyses. If dissolved or colloidal byproducts were responsible for increasing colloid lability, then one would expect to see gradually increasing lability over the time course of the incubation as more and more bacteria are consumed and byproducts accumulate, continually reacting with the colloidal $\mathrm{Fe}$ in the system. Instead, discrete peaks in colloidal Fe reactivity are observed coincident with the most intense grazing period in the culture. This points to ingestion as critical in generating reactive iron, unless dissolved by-products responsible for the reaction are exceedingly short-lived. Data from a variety of sources (Karner et. al., 1994; Nagata and Kirchman, 1992;Gonzalez et. al., 1993) indicate that the activity of enzymes released as a consequence of grazing retain their activity for several days at least. This, combined with the data in Figure 3.9, would tend to discount the possibility that extracellular enzymes could be responsible for increasing Fe colloid reactivity. It is possible that other dissolved components with very short halflives, such as reactive oxygen species which may be associated with protozoan 
phagocytosis (Stossel, 1974), could be responsible for the observed changes in colloidal Fe reactivity. There is no data to indicate that protists actually release such agents to the external medium, but our data thus far does not discount such a phenomenon as a possible mechanism for increasing colloidal Fe reactivity.

Experiments with iron-limited diatoms

Method - The data discussed previously indicate that protozoan grazers can cause a significant increase in the reactivity of colloidal ferrihydrite, as determined by the oxine and $1 \mathrm{~N} 2 \mathrm{~N}$ competitive ligand exchange techniques. Increasing oxine reactivity of colloidal $\mathrm{Fe}$ has been positively correlated with increasing bioavailability of colloidal $\mathrm{Fe}$ (Wells et. al., 1991b). In order to obtain a more direct assessment of the effect of protozoan grazing on the bioavailability of colloidal $\mathrm{Fe}$, a series of experiments was performed in which Fe-limited diatoms (Thalassiosira weissflogii, clone Actin) were used as "probes" for bioavailable iron. In laboratory culture, Fe-limited growth of $T$. weissflogii is relatively easy to achieve due to the high iron requirement of this coastal diatom. Diatoms were grown under Fe-limiting conditions by adding an excess of all necessary nutrients except $\mathrm{Fe}$ to the basal coastal seawater medium, and limiting the amount of available iron by adding $1 \mu \mathrm{M}$ of EDTA. Fe-limited diatoms grown under such conditions exhibited clear indications of reduced growth rate and reduced levels of $\mathrm{chl} a$ per cell relative to Fe-replete cultures. Therefore, in the experiments described here, diatom growth rate and $\mathrm{chl} a$ concentrations in the experimental systems relative to the controls were used as diagnostic indicators of the amount of bioavailable iron produced from the colloidal ferrihydrite via grazer-mediated processes.

Diatoms for these experiments were grown up under conditions of iron limitation, in axenic culture. Growth media was VSW with $1 \mu \mathrm{M}$ EDTA, $0.5-1 \times 10^{-7} \mathrm{M} \mathrm{Fe}$, and $\mathrm{f} / 2$ nutrients except Fe added. Background Fe concentrations in VSW were about $21 \mathrm{nM}$. These diatoms were grown in the laboratory of Don Anderson at WHOI, with the assistance of Deana Erdner. Growth conditions for iron-limiting T. weissflogii clone Actin had been well-established previously by Ms. Erdner. Prior to being used in 
experiments, the diatoms were harvested from iron-limited culture (in equal sized mixed aliquots for each model system) by gentle vacuum filtration onto $47 \mathrm{~mm}$ diameter, $3 \mu \mathrm{m}$ pore size Nuclepore polycarbonate filters, and resuspended in experimental media to achieve an initial cell density of about $1 \times 10^{4}$ cells $/ \mathrm{ml}$ in each model system. Experimental medium consisted of VSW with $1 \mu \mathrm{M}$ EDTA and $\mathrm{f} / 2$ nutrients except iron added. Thus all nutrients except iron were in excess, and the only source of iron added to the systems was the colloidal Fe. Several hours prior to the addition of the diatoms, colloidal ferrihydrite $(\sim 1 \mu \mathrm{M})$ was added to the experimental media in the model systems. Bacteria, $H$. halodurans, were added about 1 hour after the addition of the Fe colloids. Appropriate model systems were inoculated with protozoan grazers just prior to harvesting and adding the resuspended diatoms.

Specific details on model system composition, etc. accompany the descriptions of the various experiments described in this section. For a given experiment, the following types of model systems were set up, in duplicate or triplicate:

- Colloid controls - containing Fe colloids and diatoms

- Bacteria controls - containing Fe colloids, bacteria and diatoms

- Bacteria iron controls - containing bacteria, protozoans and diatoms

- Experimental systems - containing Fe colloids, bacteria, protozoans and diatoms

This combination of systems provided appropriate controls for direct effects of colloidal $\mathrm{Fe}$ on diatom growth, bacterially-mediated effects of colloidal $\mathrm{Fe}$ on diatom growth, and effects of remineralized bacterial biomass on diatom growth.

During experiments, model systems (100ml each in volume) were maintained in $250 \mathrm{ml}$ polycarbonate centrifuge bottles, at $20^{\circ} \mathrm{C}$ in an incubator without agitation except just prior to sampling. In order to facilitate diatom growth, model systems were illuminated by cool-white fluorescent light at about $100 \mu$ Einsteins $\mathrm{m}^{-2} \mathrm{~s}^{-1}$ on a $16 \mathrm{~h}$ light: $8 \mathrm{~h}$ dark cycle. Most experiments ran for about 4 days. During this time, the bottles were periodically ( $\sim$ every 24 hours) removed from the incubator, mixed, and aseptically sampled in triplicate (sample volumes $300 \mu \mathrm{leach}$ ) for diatom cell counts. These samples were preserved in Utermohl's iodine solution, and counted by light microscopy on a 
hemacytometer. Occasionally, samples preserved in $1 \%$ glutaraldehyde were taken to examine by epifluorescence microscopy for the presence of chlorophyll fluorescence in protozoan food vacuoles.

At the end of the experiment, $\mathrm{pH}$ was measured in each model system, and samples for diatom cell counts were taken. In addition, triplicate samples were taken from each bottle for determination of diatom chlorophyll concentrations. Aliquots of culture (usually $5 \mathrm{ml}$ each) were harvested onto $25 \mathrm{~mm}$ diameter, $3 \mu \mathrm{m}$ pore size Millipore cellulose acetate filters. These filters were stored at $-80^{\circ} \mathrm{C}$ until chlorophyll extraction prior to measurement. For extraction, each filter was placed in $10 \mathrm{ml}$ of cold $90 \%$ acetone and thoroughly agitated. Filters in acetone were stored in the dark in the refrigerator for 24 hours prior to chlorophyll analysis. For analysis, acetone/filter samples were vortexed, centrifuged, and the acetone decanted into clean glass test tubes. Chlorophyll $a$ fluorescence measurements were immediately taken on a Turner Designs Fluorometer. Phaeopigments were measured after acidification of the acetone extracts with 2 drops of $10 \% \mathrm{HCl}$.

Initial experiments - In the first attempt at conducting an experiment with Fe-limited diatoms as probes of bioavailable iron, Fe colloids were added at a level of $5 \mu \mathrm{M}$ iron. This experiment failed because $5 \mu \mathrm{M}$ of colloidal $\mathrm{Fe}$ was too much, causing diatoms in all cultures with added $\mathrm{Fe}$ colloids to increase in growth rate and chlorophyll. In the subsequent experiment (and in all of the others to follow), colloidal Fe was added at $1 \mu \mathrm{M}$ concentration, similar to the $1 \mathrm{~N} 2 \mathrm{~N}$ and oxine lability/size fractionation experiments.

For this experiment, the following model systems were set up:

-2 colloid controls, $50^{\circ} \mathrm{C}$ FERR

-2 colloid controls, $90^{\circ} \mathrm{C}$ FERR

- 2 bacteria controls, $50^{\circ} \mathrm{C}$ FERR

- 2 bacteria controls, $90^{\circ} \mathrm{C}$ FERR

- 2 bacterial iron controls, Cafeteria as grazer

- 2 experimental cultures, $50^{\circ} \mathrm{C}$ FERR, Cafeteria as grazer 
- 2 experimental cultures, $90^{\circ} \mathrm{C}$ FERR, Cafeteria as grazer

The bacteria for this experiment were grown up in a reduced-iron medium, with glucose as the carbon source. During harvesting and resuspension, the bacteria were resuspended twice in a saline solution with $1 \mu \mathrm{M}$ concentration of EDTA. The final rinse and resuspension was in VSW with $\mathrm{f} / 2$ nutrients (except Fe). This was done in order to reduce the amount of bioavailable $\mathrm{Fe}$ associated with the remineralization of bacterial biomass in the experiment. Bacteria were not heat-killed, and were added to the model systems at a concentration of about $3 \times 10^{7}$ cells $/ \mathrm{ml}$. Diatoms were resuspended in all model systems at an initial concentration of $1.25( \pm 0.11) \times 10^{4}$ cells $/ \mathrm{ml}$. The experiment ran 4 days.

Data on diatom cell counts and chl $a$ concentrations at the end of the experiment are shown in Table 3.1. This data set is incomplete due to problems with the preparation of some of the acetone extracts for chl $a$ analysis. Additional data taken on $\mathrm{pH}$ at the end of the experiment indicated $\mathrm{pH}$ in all cultures was 7.8. As Table 3.1 indicates, diatom cell densities in the experimental cultures at the end of the experiment were almost twice as high as in the bacteria and colloid controls, and the diatom chl $a$ concentrations per cell were almost three times as high. This is clear evidence for an increase in the amount of bioavailable iron in the experimental cultures as a result of grazer activity. There appeared to be little difference between the bacteria controls and colloid controls, or between the $50^{\circ} \mathrm{C}$ and $90^{\circ} \mathrm{C}$ ferrihydrite, in terms of diatom response. Very little bioavailable iron was evidently produced in the bacterial iron controls. This is all consistent with previous data.

A similar experiment was carried out in an attempt to collect a more complete data set on diatom growth rate and chl $a$ concentration. For this experiment $50^{\circ} \mathrm{C}$ ferrihydrite only was used. Colloid control, bacteria control, bacteria iron control, and experimental cultures were set up in triplicate, for a total of twelve bottles. Cafeteria was again the grazer. H. halodurans was grown up on normal glucose medium, without any special attempts to limit its iron content during growth or rinsing, and cells were not heat-killed. Bacteria were added at $t_{0}$ at a concentration of about $2 \times 10^{7}$ cells $/ \mathrm{ml}$. 
Table 3.1 - Results from initial experiment with diatoms as probes of bioavailable iron. Data shown for diatom cell concentrations and chl a concentrations per cell in various model systems at the end of the experiment, day 4.

Values shown are the average and standard deviation of replicate bottles.

\begin{tabular}{|c|c|c|c|}
\hline Model Systen & & Diatom cells/ml $\left(\times 10^{4}\right)$ & pg chl $a /$ diatom c \\
\hline $\begin{array}{l}\text { bacterial iron } \\
\text { control }\end{array}$ & & $1.83 \pm 0.08$ & $0.11 \pm 0.001$ \\
\hline colloid & $50^{\circ} \mathrm{C}$ FERR & $3.77 \pm 0.56$ & ND \\
\hline control & $90^{\circ} \mathrm{C}$ FERR & $4.08 \pm 0.15$ & $\mathrm{ND}$ \\
\hline $\begin{array}{r}\text { bacteria } \\
\text { control }\end{array}$ & $\begin{array}{c}50^{\circ} \mathrm{C} \text { FERR } \\
90^{\circ} \mathrm{C} \text { FERR }\end{array}$ & $\begin{array}{c}4.02 \pm 0.49 \\
3.15 \pm 0.23\end{array}$ & $\begin{array}{r}* 0.12 \pm 0.02 \\
0.15 \pm 0.04\end{array}$ \\
\hline $\begin{array}{l}\text { experimental } \\
\text { systems }\end{array}$ & $\begin{array}{l}50^{\circ} \mathrm{C} \text { FERR } \\
90^{\circ} \mathrm{C} \text { FERR }\end{array}$ & $\begin{array}{l}6.56 \pm 0.02 \\
7.21 \pm 0.12\end{array}$ & $\begin{array}{l}0.41 \pm 0.05 \\
0.38 \pm 0.02\end{array}$ \\
\hline $\mathrm{ND}=$ no data & obtained & & \\
\hline
\end{tabular}


Data on $\mathrm{pH}$ taken at the end of this experiment (day 4) indicated higher $\mathrm{pH}$ in the experimental cultures $(\sim 8.9)$ than in the controls ( $~ 8.6$ in colloid controls, 8.3 in bacteria controls, and 8.0 in bacteria iron controls). The variations in $\mathrm{pH}$ correlated well with variations in diatom biomass, as seen in Figure 3.10 which shows data on diatom growth rates and chl $a$ concentrations in this experiment. Again, there was a clear indication of production of bioavailable iron from the colloidal ferrihydrite in the experimental culture. Chlorophyll concentrations were about twice as high, and diatom cell yields were almost three times as high as in controls. The colloids did appear to provide some available iron to the diatoms without the presence of the protozoans, but protozoans clearly enhanced the supply. The presence of bacteria in the bacteria controls did not appear to enhance the amount of iron available to the diatoms relative to that in the colloid controls.

Compared to the data in Table 3.1, these diatoms had about ten times as much chlorophyll fluorescence per cell as the diatoms in the previous experiment. This was because they were grown up under less severe conditions of iron stress. This may explain their somewhat greater growth response relative to the diatoms in the previous experiment. The growth curves in Figure 3.10 reveal that there was a lag phase of about two days before the diatoms in the experimental culture began to increase in growth rate. The period of active protozoan grazing was completed by about 48 hours, as previous data has shown. Diatoms evidently sequester available iron during this lag phase for the first 48 hours, and subsequently utilize it to fuel a growth spurt during the latter half of the experiment.

This is consistent with what has been observed in iron addition experiments in the field, where lag phases are commonly observed in iron-amended bottles (or water patches, as in IRONEX I and II) prior to increases in phytoplankton biomass (Martin et. al., 1989,1990; Coale et. al. 1996). The length of diatom lag phase and degree of growth response may be dependent on their physiological state at the beginning of the experiment, with more severely-limited populations taking longer to increase growth rate. The rate of chlorophyll response may be faster - note that chlorophyll levels in the experimental cultures of this experiment were twice as high as controls, whereas in the 

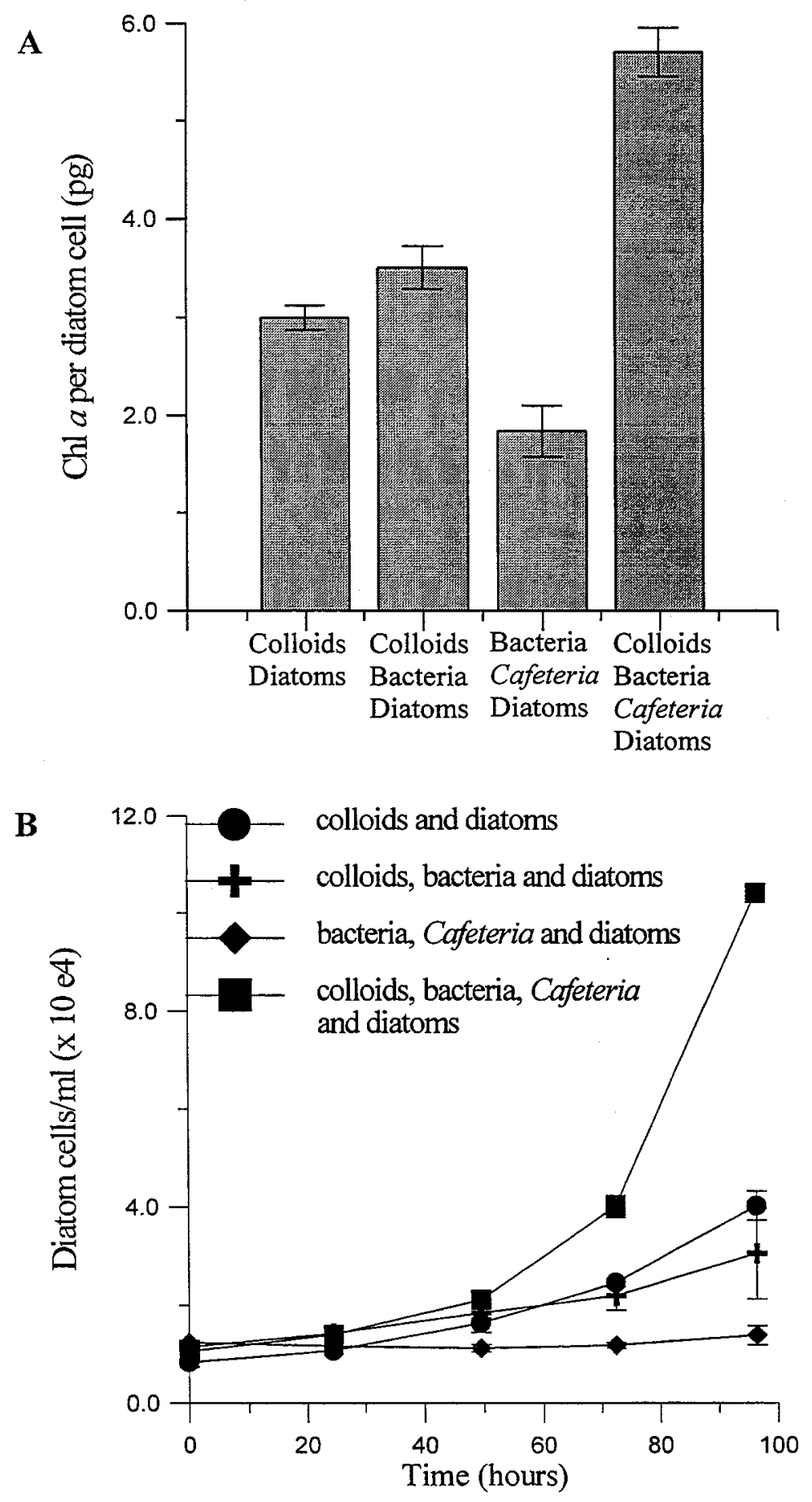

Figure 3.10 - Data from second iron-limited diatom experiment using live bacteria as prey.

A. chl a fluorescence per diatom cell at $t=4$ days

B. diatom growth curves - cell concentration vs time 
previous experiment the difference was closer to a factor of three. Diatoms may respond to relief of iron limitation by first producing more chlorophyll on a single cell basis and then increasing division rates, tending to increase cell concentration but lower chlorophyll concentration per cell.

In both of these experiments, fresh samples preserved in $1 \%$ glutaraldehyde were examined by epifluorescence microscopy in order to see if there was any chlorophyll fluorescence associated with grazer food vacuoles. This was done to ensure that the protozoan grazers in the system (Cafeteria) were not consuming diatom cells. No fluorescing protists were detected. Consumption of diatoms by the protists in these systems is unlikely, given that the diatoms are $10-15 \mu \mathrm{m}$ in size, while the microflagellate Cafeteria is only $2-4 \mu \mathrm{m}$ in size. Some microflagellates, like Paraphysomonas imperforata, have been reported to ingest similarly-sized diatoms as prey (Goldman and Caron, 1985). This is not the case for Cafeteria, which is significantly smaller than Paraphysomonas. In any case, if consumption of diatoms by protozoans played a significant role in increasing the supply of bioavailable iron to the diatoms, such an effect should show up in the bacteria iron controls.

Diatom experiments with heat-killed bacteria - As mentioned in the section on 1N2N experiments, some concern was raised about the role played by bacteria in increasing colloidal iron reactivity. Since, as previously explained, experiments with heat-killed bacteria were not practical by CLE/CSV with $1 \mathrm{~N} 2 \mathrm{~N}$, heat-killed bacteria experiments were conducted using Fe-limited diatoms as probes for the production of bioavailable $\mathrm{Fe}$. Although care was taken to prevent contamination of these experiments by live bacteria, it should be noted that, since the experiments ran for 4 days, it is possible that some population of live bacteria could have developed by the end of the experiment. Therefore the use of heat-killed bacteria in these experiments cannot be said to have completely eliminated the possible effects of live bacteria. The $H$. halodurans in the experiments described here were grown up on yeast-extract media, heat-killed by incubation in a water bath at $60^{\circ} \mathrm{C}$ for 2 hours, and then rinsed and resuspended $3 \mathrm{x}$ via centrifugation 
prior to being added to experimental model systems. In all other respects, the set-up of the two experiments described in this section is similar to the previous section.

The first experiment with heat-killed bacteria involved the following cultures, each type set up in triplicate: colloid controls; bacteria controls; bacteria iron controls with Cafeteria as grazer; bacteria iron controls with Pteridomonas as grazer; experimental cultures with Cafeteria as grazer; experimental cultures with Pteridomonas as grazer. Pteridomonas sp. (clone NB1 from D. Caron's culture collection) is a small microflagellate similar in size and morphology to Cafeteria. Prior to the experiment, both Cafeteria and Pteridomonas were repeatedly transferred into cultures of heat-killed, resuspended yeast-extract-grown bacteria. The Cafeteria inoculum used in the experiment had been transferred into heat-killed cultures $4 \mathrm{x}$; the Pteridomonas inoculum had been transferred $3 x$.

Some difficulty was experienced during experimental set-up with harvesting and resuspension of the diatoms, which were at a lower density than usual in the initial Felimiting culture. The diatoms were under a high degree of iron stress, as indicated by their low cell density and lack of pigmentation. This lack of color made resuspending the diatoms into the experimental model systems difficult, because the cells on the filter were difficult to see, and therefore difficult to completely rinse off. As a result of this, the diatom concentrations in the experimental model systems were at densities too low to feasibly obtain a cell count by light microscopy with a haemacytometer. This was determined on day four of the experiment, so the experiment was run an extra 24 hours, until day 5, at which point the experiment was terminated and samples taken for chlorophyll fluorescence determinations. Because cell densities were still so low, $20 \mathrm{ml}$ of culture was filtered for each chlorophyll sample, as opposed to $5 \mathrm{ml}$ in previous experiments. Cell counts were not obtained.

Table 3.2 presents the results of the chlorophyll analyses for this experiment. Because cell concentrations were not ascertained, only bulk chlorophyll measurements in terms of fluorescence units are presented, normalized to the same volume in all samples. It is apparent that the chlorophyll $a$ fluorescence was much higher in the Cafeteria 
Table 3.2 - Bulk chlorophyll $a$ measurements from first diatom experiment with heat-killed bacteria, shown for different model systems at the end of the experiment (day 5). Values are normalized to $20 \mathrm{ml}$ of culture extracted for chlorophyll analysis. Values shown are the average and standard deviation of triplicate bottles.

\section{Model Sytem}

colloid control

bacteria control

bacteria iron control

(Cafeteria)

bacteria iron control

(Oikomonas)

experimental system

(Cafeteria)

experimental system

(Oikomonas)

\section{Chlorophyll $a$ fluoroscence (fu)}

$8.41 \pm 3.05$

$6.62 \pm 2.06$

$3.58 \pm 0.75$

$4.59 \pm 1.09$

$215.47 \pm 32.35$

$81.41 \pm 1.66$ 
experimental cultures than in the controls, by about a factor of 30 . Fluorescence in the Pteridomonas experimental cultures was also significantly higher than the controls, by about a factor of 12 .

While these differences are quite large relative to the chl $a$ results presented previously, it must be remembered that this is a bulk chlorophyll $a$ fluorescence measurement, whereas the previous chlorophyll data was normalized on a per diatom cell basis. Bulk fluorescence is influenced both by increases in diatom chl $a$ per cell, and also by increases in diatom cell concentration. Thus, differences in bulk chlorophyll should be larger than differences in chlorophyll per cell. In the data shown in Figure 3.10, for example, the difference between experimental and control cultures in terms of chl $a$ concentration per diatom cell is about a factor of 2 , but the difference in these cultures in terms of bulk chlorophyll $a$ fluorescence (for equal volumes of culture) was about a factor of 5 (data not shown). Factors of 30 and 12 are still relatively large, however, so it seems that both Cafeteria and Pteridomonas had a large effect in increasing the supply of bioavailable iron to the diatoms in this experiment, particularly Cafeteria. The effect may have been exacerbated relative to previous experiments both because of the lower concentrations of diatoms in this experiment (less competition between cells for the same amount of bioavailable $\mathrm{Fe}$ ), and because this experiment was run for five days instead of the usual four. It is interesting to note the interspecies difference between Cafeteria and Pteridomonas, which confirms previous findings (using different techniques) of interspecies differences in the effect of protists on colloidal ferrihydrite.

In order to obtain a more complete data set in a diatom experiment with heat-killed bacteria, another experiment was conducted similar to the one previously described, but involving only Cafeteria as the grazer. The Cafeteria inoculum in this experiment had been transferred into heat-killed bacterial culture about 10 times in succession prior to the experiment. Diatom cells in this experiment were grown up under conditions of iron limitation, but less extreme than in the previous experiment, so the resuspension step was easier. H. halodurans was again grown on yeast extract and heat-killed, rinsed $3 \mathrm{x}$, and resuspended prior to the experiment. Model systems were set up in triplicate for this 
experiment and included the following types: colloid controls; bacteria controls; bacteria iron controls with Cafeteria; and experimental cultures with Cafeteria.

The results of this experiment for both diatom chlorophyll $a$ fluorescence per cell and diatom growth curves are shown in Figure 3.11. It can be seen that these results are very similar to those shown in Figure 3.10, indicating that whether the bacterial prey were alive or dead apparently made very little difference to the eventual result. The experiment with heat-killed bacteria even seemed to show slightly greater enhancement of diatom growth and chlorophyll in the experimental cultures. The production of bioavailable iron from the colloidal ferrihydrite in the experimental cultures was apparently due to the ingestion and subsequent dissolution of colloidal iron by protozoan grazers. Any action of the bacteria on the Fe colloids played only a minor role, if at all, in this process.

\section{Conclusion}

\section{$\underline{\text { Summary }}$}

In this chapter, four independent techniques have been employed to investigate the effect of protozoan grazing on colloidal ferrihydrite in laboratory culture systems. Methods used included: Size fractionation of radio-labeled Fe colloids in systems with EDTA as a dissolved Fe trap; oxine lability with radio-labeled Fe colloids; CLE/CSV with $1 \mathrm{~N} 2 \mathrm{~N}$; and Fe-limited diatoms as probes of bioavailable Fe. The results obtained from these four different techniques indicate that grazing by heterotrophic protists in the nanoplanktonic size class can cause dissolution of colloidal ferrihydrite, thereby increasing the reactivity and bioavailablity of this iron oxide phase.

Data indicates that the effect of the protozoan grazers on iron colloid lability is independent of medium $\mathrm{pH}$. It has also been shown that the medium of an actively grazing protozoan culture from which protists have been removed by $1 \mu \mathrm{m}$ filtration does not affect ferrihydrite reactivity to the same extent as whole culture containing protozoan grazers. The peak rate of production of reactive iron in these systems appears to correlate strongly with peak grazing rates, when the growth rate of protists and the consumption 

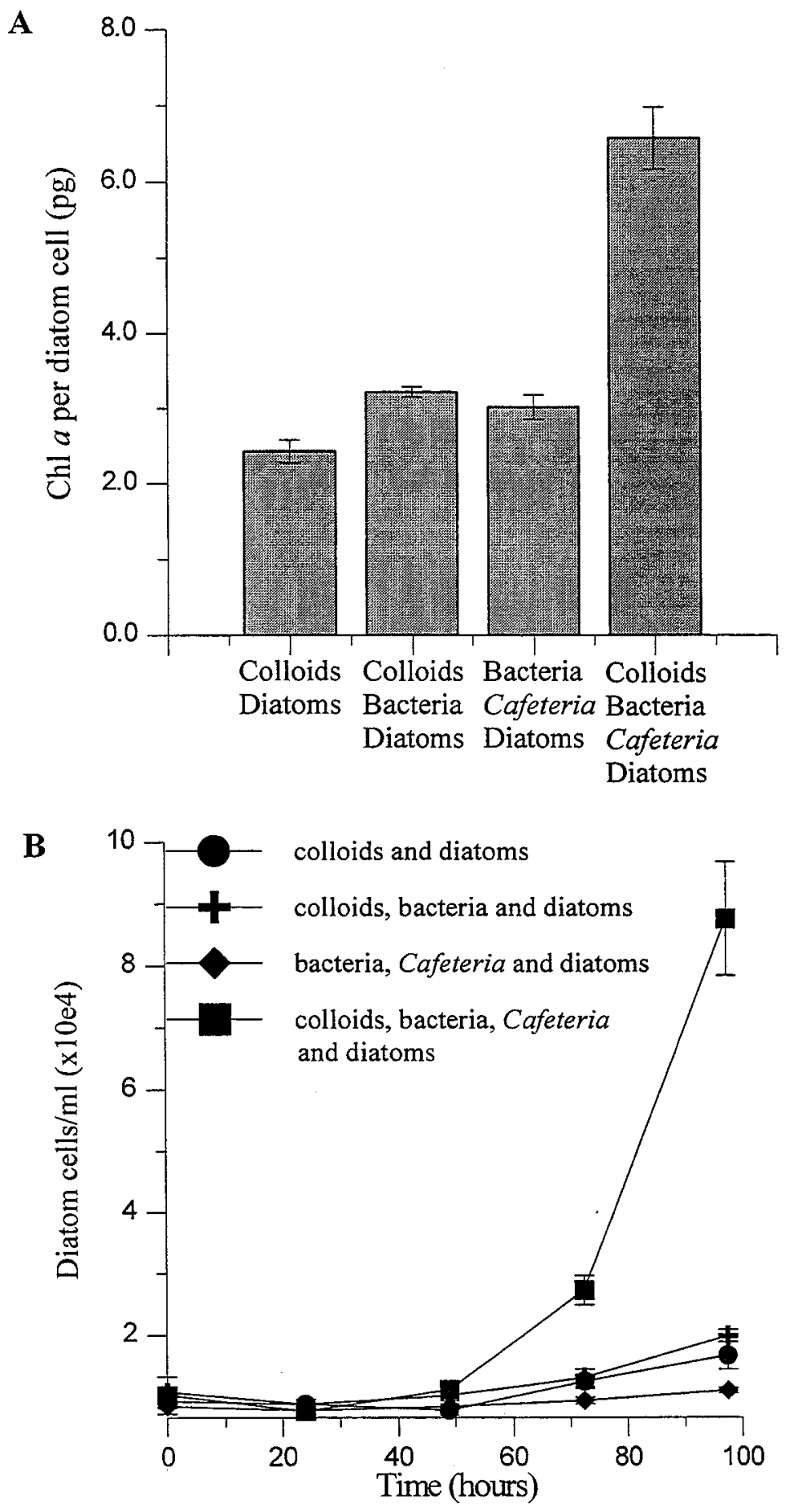

Figure 3.11 - Data from second iron-limited diatom experiment using heat-killed bacteria as prey.
A. chl a fluorescence per diatom cell at $t=4$ days
B. diatom growth curves - cell concentration vs time 
rate of bacteria is maximal. These results support the conclusion that the effect of protozoan grazers on colloidal ferrihydrite is due to the ingestion and chemical transformation of $\mathrm{Fe}$ colloids within the low- $\mathrm{pH}$, enzyme-rich microenvironment of the protozoan food vacuole. While the data presented here has demonstrated that several different protozoan species can mediate transformations of colloidal iron, the extent to which such transformations occur apppears to be relatively species-specific. This may be related to differences in the digestive physiology of various protozoan species, such as vacuole residence time, acidity, enzymatic activity, etc.

\section{Significance and Caveats}

In discussing the significance of these results it is necessary to consider the various caveats involved in trying to extrapolate from laboratory model systems to natural systems. One obvious caveat involves the higher concentrations of protists, bacteria, and colloidal iron in our model systems relative to natural marine waters. Because of the concentrated nature of the model systems, the rate and extent of the processes occurring within tend to be magnified over what would be observed in a more dilute environment. The mechanism(s) responsible for the observed processes, however, should be the same in both model and natural systems.

With mechanistic knowledge based on our studies of model systems, it is possible to make a rough approximation of the rate of protozoan-mediated dissolution of colloidal iron in the oceans. Given an average volume clearance rate for microflagellates as determined by various studies $\left(2 \times 10^{-6} \mathrm{ml} \mathrm{cell}^{-1} \mathrm{~h}^{-1}\right.$, Berninger et. al., 1991), along with a typical concentration of microflagellate cells in open ocean waters $\left(10^{3}\right.$ cells per $\mathrm{ml}$, Berninger et. al., 1991), these two constants can be multiplied to derive a first-order rate constant for the dissolution of colloidal iron by protozoan grazers of $2 \times 10^{-3} \mathrm{~h}^{-1}$. This is only a rough estimate, and assumes a dissolution efficiency of $100 \%$ on the part of the protists. Bearing this in mind, it is nonetheless significant to note that this rate constant is approximately one-tenth as large as one published value for the maximum (noon-time) near-surface first-order rate constant for the photoreduction of colloidal iron in seawater, 
$1.8 \times 10^{-2} \mathrm{~h}^{-1}$ (Wells and Mayer, 1991a). This photoreduction rate, as indicated, is a maximum. The photoreduction rate declines exponentially with depth as a result of light attenuation (Wells et. al., 1991), and is also well below maximum for much of the day, on cloudy days, and of course at night. Protozoan grazers occur throughout the upper water column (and below), and are continually active. Thus, when integrated temporally over the entire euphotic zone, protozoan grazing may equal or exceed photoreduction as a mechanism for converting non-labile forms of iron into reactive, bioavailable forms.

"Non-labile forms of iron" were represented in our model systems by synthetic ferrihydrite colloids. Although phases like this have often been used in similar studies of the influence of photoreductive processes on iron speciation in the marine environment (eg. Wells and Mayer, 1991a; Johnson et. al., 1994), this brings up an additional caveat inherent in the use of model systems: the degree to which the various components of a model system are representative of their analogous components in the natural environment. The colloidal ferrihydrite used in these studies differs from naturallyoccurring iron colloids in that it was synthesized as a relatively pure inorganic phase. Colloidal iron in seawater most likely occurs in association with a primarily organic matrix (Wells et. al., 1995). Even primarily inorganic iron oxides formed in natural systems often contain substantial quantities of organic and inorganic impuritities, and these substances can have an effect on iron oxide reactivity (Deng and Stumm, 1994).

The pool of iron occuring as small particles and colloids in natural systems is likely to be quite heterogeneous. No consensus currently exists on the chemical composition of this iron pool, particularly in marine systems. Iron oxyhydroxides, such as the ferrihydrite employed in our experiments, are at least known to occur in the marine environment, particularly in coastal waters (Wells and Goldberg, 1992). In terms of their chemical reactivity, our synthetic phases are similar to naturally-occurring iron solids studied by Wells and Mayer, 1991b. While synthesized as an inorganic phase, the colloidal ferrihydrite in our model systems is undoubtedly intimately associated with organic material as a result of the presence of relatively high concentrations of bacteria, protozoan grazers, and dissolved organic carbon. In these respects, then, the colloidal iron 
used in our experiments is likely to be a reasonable facsimile of naturally-occurring, relatively refractory iron solids.

\section{Protozoan grazers and "new" iron}

At the beginning of this chapter, the operational classification of iron as "bioavailable" (reactive) vs. "non-bioavailable" (non-reactive) was discussed. Accepting that the colloidal ferrihydrite used in these experiments represents an appropriate model for nonbioavailable iron solids, and that protozoan grazers can potentially play an important role in converting those solids into more bioavailable forms, under what circumstances is this process likely to be significant? In the open ocean, most of the solid iron is likely to occur in the form of living organinsms. Protozoans are already thought to be important in the remineralization and recycling of biological iron, particularly in HNLC oligotrophic regimes dominated by small phytoplankton cells (Landry et. al, 1997; Hutchins et. al., 1993).

In HNLC areas, relatively refractory particulate iron entering into the system from external sources can be an important parameter in determining the amount of new production in the system (Landry et. al., 1997). A key intermediate step in converting "new" iron into new production is, of course, the conversion of the new iron into a form that is biologically available. Protozoan grazers may contribute to this process.

In the central equatorial Pacific, for example, recent studies have shown that the most important source of new iron to the system is from the upwelling of water from the Equatorial Undercurrent (Gordon et. al., 1997). The iron in this water is believed to be of lithogenic origin, derived from hydrothermal vents in the eastern Pacific near the source waters of the Equatorial Undercurrent. As a hydrothermal precipitate, this iron must be largely composed of small particles and colloids of amorphous iron oxyhydroxides (Campbell, 1991). This source of new iron upwells from a depth of about $175 \mathrm{~m}$, and both particulate and dissolved/colloidal $(<0.4 \mu \mathrm{m})$ iron is drawn down to background levels by a depth of $100 \mathrm{~m}$, at the base of the euphotic zone (Gordon et. al., 1997). At these depths, rates of colloidal iron oxide dissolution due to photolysis are negligible, 
even in clear ocean waters (Wells et. al., 1991a). In the absence of photochemical processes, dissolution of small particles and colloids of iron oxide within the food vacuoles of the protozoan grazers known to be important in this ecosystem may be an important vector for the entry of new iron into the biologically active pool of iron in the central Equatorial Pacific. A similar situation may exist in the Southern Ocean, where upwelling of iron derived from the benthic nepheloid layer and from the diagenetic altering of shelf sediments is thought to be the main source of iron to the Antarctic Circumpolar Current (Loescher et. al., 1997).

In addition to HNLC regions, recent evidence indicates that new iron inputs may play an important role in the biological production of coastal regions (Johnson et. al., 1997). This may be particularly true in the coastal California upwelling region, which has been the object of a time-series study based at MBARI since 1992 (Chavez, 1996). In this regime, offshore waters appear to become depleted in iron before nitrate. Additional data indicate that particulate iron is solubilized and consumed by biota in surface waters (Johnson et. al., 1997). Particulate iron may in fact play a major role in determining the available supply of iron for primary producers in this system. The bulk of iron input to these waters occurs as suspended sediment particles in fluvial discharge, during the late winter to early spring. New iron supply to phytoplankton during upwelling season in the late spring and summer may be largely mediated by diagnetic re-working of shelf sediments and upwelling of sediment-derived particulate, colloidal, and dissolved iron (Ken Bruland, personal communication). In this coastal system where photochemicallyreactive light is likely to be attenuated at relatively shallow depths (Wells et. al., 1991a), protozoan-mediated reaction pathways as discussed in this chapter may be important in increasing the bioavailability of particulate and colloidal forms of new iron. 


\section{References}

- Anderson, D.M. and F.M.M. Morel. 1978. Copper sensitivity of Gonyaulax tamarensis. Limnol. Oceanogr. 23: 283-295.

- Berninger, U.-G., D.A. Caron, R.W. Sanders and B.J. Finlay. 1991. Heterotrophic flagellates of planktonic communities: their characteristics and methods of study. p. 3956. In The Biology of Free-living Heterotrophic Flagellates, Systematics Association Special Volume 45. Patterson, D.J. and J. Larsen (eds.). Clarendon, Oxford.

- Campbell, A.C. 1991. Mineralogy and chemistry of particles by synchrotron X-ray, Mossbauer and plasma MS. p. 375-390. In Marine Particles: Analysis and Characterization. Hurd, D.C. and D.W. Spencer, eds. Geophysical Monograph 63. AGU, Washington, D.C.

- Chavez, F.P. 1996. Forcing and biological impact of onset of the 1992 El Nino in central California. Geophys. Res. Lett. 23: 265-268.

- Coale, K.H. and others. 1996. A massive phytoplankton bloom induced by an ecosystem-scale iron fertilization experiment in the equatorial Pacific Ocean. Nature 383: 495-501.

- Cornell, R.M. and U. Schwertmann. 1996. The Iron Oxides: Structure, Properties, Reactions, Occurrence and Uses. VCH, New York, NY.

- Crumbliss, A.L. 1991. Aqueous solution equilibrium and kinetic studies of iron siderophore and model siderophore complexes. Ch. 7. In CRC Handbook of Microbial Iron Chelates. Winkelmann, G. (ed.) CRC Press, New York, NY.

- de Baar, H.J.W., A.G.J. Buma, R.F. Nolting, G.C. Cadee, G. Jacques and P.J. Treguer. 1990. On iron limitation of the Southern Ocean: Experimental observations in the Wedell and Scotia Seas. Mar. Ecol. Prog. Ser. 65: 105-122.

- Deng, Y. and W. Stumm. 1994. Reactivity of aquatic iron(III) oxyhydroxides implications for redox cycling of iron in natural waters. Appl. Geochem. 9: 23-36. - Fenchel, T. 1987. Ecology of Protozoa: The Biology of Free-living Phagotrophic Protists. Madison: Science Tech Publishers. 
- Goldman, J.C. and Caron, D.A. 1985. Experimental studies on an omnivorous microflagellate: implications for grazing and nutrient regeneration in the marine microbial food chain. Deep-Sea Res. 32: 899-915.

- Gonzalez, J.M., B.F. Sherr and E.B. Sherr. 1993. Digestive enzyme activity as a quantitative measure of protistan grazing: the acid lysozyme assay for bacterivory. Mar. Ecol. Prog. Ser. 100: 197-206.

- Gordon, R.M., K.H. Coale and K.S. Johnson. 1997. Iron distributions in the equatorial Pacific: Implications for new production. Limnol. Oceanogr. 42: 419-431.

- Gu, X.-Y. and P.H. Hsu. 1987. Hydrolytic formation of submicron iron(III) oxides from dilute ferric nitrate solutions. Soil Sci. Am. J. 51: 469-474.

- Haygoood, M.G., P.D. Holt and A. Butler. 1993. Aerobactin production by a planktonic marine Vibrio sp. Limnol. Oceanogr. 38: 1091-1097.

- Hering, J. and F.M.M. Morel. 1988. Kinetics of trace metal complexation: Role of alkaline-earth metals. Environ. Sci. Technol. 22: 1469-1478.

- Hudson, R.J.M. and F.M.M. Morel. 1989. Distinguishing between extra- and intracellular iron in marine phytoplankton. Limnol. Oceanogr. 34: 1113-1120.

- Hutchins, D.A. and K.W. Bruland. 1994. Grazer-mediated regeneration and assimilation of Fe, $\mathrm{Zn}$ and $\mathrm{Mn}$ from planktonic prey. Mar. Ecol. Prog. Ser. 110: 259-269.

- Hutchins, D.A., G.R. DiTullio and K.W. Bruland. 1993. Iron and regenerated production: Evidence for biological iron recycling in two marine environments. Limnol. Oceanogr. 38: 1242-1255.

- Johnson, K.S., K.H. Coale, V.A. Elrod and N.W. Tindale. 1994. Iron photochemistry in the equatorial Pacific. Mar. Chem. 46: 319-334.

- Johnson, K.S., R.M. Gordon and K.H. Coale. 1997. What controls dissolved iron concentrations in the world ocean? Mar. Chem. 57: 137-161.

- Karner, M., C. Ferrier-Pages and F. Rassoulzadegan. 1994. Phagotrophic nanoflagellates contribute to occurrence of $\alpha$-glucosidase and aminopeptidase in marine environments. Mar. Ecol. Prog. Ser. 114: 237-244. 
- Landry, M.R. and others. 1997. Iron and grazing constraints on primary production in the central equatorial Pacific: An EqPac synthesis. Limnol. Oceanogr. 42: 405-418. - Loescher, B.M., H.J.W. de Baar, J.T.M. de Jong, C. Veth and F. Dehairs. 1997. The distribution of Fe in the Antarctic Circumpolar Current. Deep-Sea Res. 44: 143-187.

- Maldonado, M.T. and N.M. Price. 1996. Influence of N substrate on Fe requirements of marine centric diatoms. Mar. Ecol. Prog. Ser. 141: 161-172.

- Martin J.H. and S.E. Fitzwater. 1988. Iron deficiency limits phtoplankton growth in the north-east Pacific subarctic. Nature. 331:341-343.

- Martin, J.H. and others. 1994. Testing the iron hypothesis in ecosystems of the equatorial Pacific Ocean. Nature 371: 123-129.

- Martin, J.H. and R.M. Gordon. 1988. Northeast Pacific iron distributions in relation to phytoplankton productivity. Deep-Sea Res. 35: 177-196.

- Martin, J.H., R.M. Gordon, S. Fitzwater and W.W. Broenkow. 1989. Vertex: phytoplankton/iron studies in the Gulf of Alaska. Deep-Sea Res. 36: 649-680. - Martin, J.H., S.E. Fitzwater and R.M. Gordon. 1990. Iron deficiency limits phytoplankton growth in Antarctic waters. Global Biogeochem. Cycl. 4: 5-12. - Matzanke, B.F., G. Muller-Matzanke and K.N. Raymond. 1989. Siderophore-mediated iron transport. Ch. 1. In Iron Carriers and Iron Proteins. Loehr, R.M. (ed.) UCH, New York, NY.

- Morel, F.M.M. 1983. Principles of Aquatic Chemistry. Wiley, New York, NY.

- Nagata, T. and D.L. Kirchman. 1990. Filtration-induced release of dissolved free amino acids: application to cultures of marine protozoa. Mar. Ecol. Prog. Ser. 68: 1-5.

- Nagata, T. and D.L. Kirchman. 1992. Release of macromolecular organic complexes by heterotrophic marine flagellates. Mar. Ecol. Prog. Ser. 83: 233-240.

- Neilands, J.B. 1974. Iron and its role in microbial physiology. p. 3-34. In Microbial Iron Metabolism: A Comprehensive Treatise. Neilands, J.B. (ed.) Academic, New York, NY.

- Reid, R.T., D.H. Live, D.J. Faulkner and A. Butler. 1993. A siderophore from a marine bacterium with an exceptional affinity constant. Nature 366: 455-458. 
- Reuter, J.G., Hutchins, D.A., Smith, R.W. \& Unsworth, N.L. Iron nutrition of Trichodesmium. In Marine pelagic cyanobacteria: Trichodesmium and other diazotrophs. Carpenter, E.J., D.G. Capone and J.G. Reuter (eds). Kluwer Academic Publishers, Boston, 289-306 (1992).

- Rich, H.W. and F.M.M. Morel. 1990. Availability of well-defined iron colloids to the marine diatom Thalassiosira weissflogii. Limnol. Oceanogr. 35: 652-662.

- Rue, E.L. and K. W. Bruland. 1995. Complexation of iron(III) by natural organic ligands in the Central North Pacific as determined by a new competitive ligand equilibration/adsorptive cathodic stripping voltammetric method. Mar. Chem. 50: 117138.

- Schwertmann, U. and R.M. Cornell. 1991. Iron Oxides in the Laboratory. VCH, New York, NY.

- Soria-Dengg, S. and U. Horstmann. 1995. Ferrioxamines B and E as iron sources for the marine diatom Phaeodactylum tricornutum. Mar. Ecol. Prog. Ser. 127: 269-277.

- Stossel, T.P. 1974. Phagocytosis [first of 3parts]. N. Engl. J. Med. 290: 717-723.

- Sulzberger, B. and H. Laubscher. 1995. Reactivity of various types of iron(III) (hydr)oxides towards light-induced dissolution. Mar. Chem. 50: 103-115.

- Sunda, W.G. and R.R.L. Guillard. 1976. The relationship between cupric ion activity and the toxicity of copper to phytoplankton. J. Mar. Res. 34: 511-529.

- van den Berg, C.M.G. 1995. Evidence for organic complexation of iron in seawater. Mar. Chem. 50: 139-157.

- Van Leeuwe, M.A., R. Scharek, H.J.W. de Baar, J.T.M. de Jong and L. Goyens. 1997. Iron enrichment experiments in the Southern Ocean: Physiological responses of plankton communities. Deep-Sea Res. 44: 189-207.

- Voelker, B.M. and D.L. Sedlak. 1995. Iron reduction by photoproduced superoxide in seawater. Mar. Chem. 50: 93-102.

- Waite, T.D. and F.M.M. Morel. 1984a. Ligand exchange and fluorescence quenching studies of the fulvic acid - iron interaction: effects of $\mathrm{pH}$ and light. Anal. Chim. Acta 162: $263-274$. 
- Waite, T.D. and F.M.M. Morel. 1984b. Photoreductive dissolution of colloidal iron oxides in natural waters. Environ. Sci. Technol. 18: 860-868.

- Wells, M.L. and E.D. Goldberg, 1992. Marine submcron particles. Mar. Chem. 40: 518.

- Wells, M.L. and L.M. Mayer. 1991a. The photoconversion of colloidal iron hydroxides in seawater. Deep-Sea Res. 38: 1379-1395.

- Wells, M.L. and L.M. Mayer. 1991b. Variations in the chemical lability of iron in estuarine, coastal and shelf waters and its implications for phytoplankton. Mar. Chem. 32: $195-210$.

- Wells, M.L., L.M. Mayer, O.F.X. Donard, M.M. de Souza Sierra and S. Ackleson. 1991a. The photolysis of colloidal iron in the oceans. Nature 353: 248-250.

- Wells, M.L., L.M. Mayer and Guillard, R.R.L. 1991b. A chemical method for estimating the availability of iron to phytoplankton in seawater. Mar. Chem. 33: 23-40.

- Wells, M.L., N.G. Zorkin and A.G. Lewis. 1983. The role of colloid chemistry in providing a source of iron to phytoplankton. J. Mar. Res. 41: 731-746.

- Wells, M.L., N.M. Price and K.W. Bruland. 1995. Iron chemistry in seawater and its relationship to phytoplankton: A workshop report. Mar. Chem. 48: 157-182.

- Wilhelm, S.W. and C.G. Trick. 1994. Iron-limited growth of cyanobacteria - multiple siderophore production is a common response. Limnol. Oceanogr. 39: 1979-1984.

- Winkelmann, G., D. v.d. Helm and J.B. Neilands. 1987. In Iron Transport in Microbes, Plants and Animals. UCH, New York, NY.

- Wu, J. and G.W. Luther. 1995. Complexation of Fe(III) by natural organic ligands in the Northwest Atlantic Ocean by a competitive ligand equilibration method and kinetic approach. Mar. Chem. 50: 159-177. 


\section{CHAPTER 4 \\ Development and application of a novel technique \\ to study reactions of synthetic iron oxides in seawater}

\section{Introduction}

\section{Iron oxides in surface waters}

Iron oxides are an abundant and widespread mineral phase in the natural environment, known to occur in soils, rocks, aquatic systems, and in the atmosphere. In surface waters, iron (hydr)oxides participate in several environmentally significant processes. Due to their finely dispersed nature and large surface area, iron oxides are an important carrier phase for surface-active organic and inorganic compounds in natural waters (Cornell and Schwertmann, 1996). Redox reactions at the iron oxide surface can result in the oxidation of reduced compounds like organic carbon, and the production of reduced iron (Fe(II)) (Suter et. al., 1991). In the presence of suitable oxidants at circum-neutral $\mathrm{pH}$, $\mathrm{Fe}(\mathrm{II})$ is rapidly re-oxidized to form amorphous iron oxide precipitates, phases which are more soluble and therefore more reactive than crystalline iron oxides, in terms of both biological uptake and/or further redox cycling (Rich and Morel, 1990).

These processes are important to the geochemical cycling of organic carbon, both as an oxidation pathway for dissolved organic compounds and as a source of iron, a limiting nutrient, to primary producers (eg. Zepp et. al., 1992; Martin et. al., 1991). Factors which influence the redox cycling of iron in surface waters are thus of interest to researchers in a variety of disciplines, from environmental chemistry to plankton ecology. As a consequence, chemical reactions of iron oxides in aquatic systems have been an active area of research. Marine surface waters are a particularly challenging environment for such research, because in oxygenated seawater the rapid reoxidation of Fe(II) and the relatively rapid reorganization of amorphous iron precipitates into more thermodynamically stable forms make it difficult to determine the actual forward reaction rate of iron oxides (Wells and Mayer, 1991a). This chapter presents details of the 
development and some applications of a novel technique to study dissolution reactions of synthetic iron oxides in seawater. Methods employed previously are briefly reviewed below.

Methods for studying reactions of iron oxides in seawater

Some of the first work on the reactivity of iron oxides in natural waters (including seawater) was done by Waite and Morel, 1984 (a,b). These authors utilized iron oxides labeled with ${ }^{59} \mathrm{Fe}$, combined with ultrafiltration, to study the production of soluble $\mathrm{Fe}$ (II) species from iron oxide phases upon irradiation of aqueous solutions under different conditions of solution $\mathrm{pH}$ and chromophore concentration. This approach was successful at low $\mathrm{pH}(\mathrm{pH} 4)$, but led to the underestimation of $\mathrm{Fe}(\mathrm{II})$ production rates above $\mathrm{pH} 6$ due to the strong $\mathrm{pH}$ dependence of the reoxidation rate of Fe(II) (Moffett and Zika, 1987). At circumneutral $\mathrm{pH}$, rapid reoxidation of $\mathrm{Fe}(\mathrm{II})$ and re-precipitation as $\mathrm{Fe}(\mathrm{III})$ reduced the amount of Fe(II) produced by photoreductive processes to levels below the detection limit of ultrafiltration.

In additional work on iron redox dynamics in seawater, Waite and Morel (1984c) utilized a controlled potential coulometric procedure combined with a masking technique in order to determine the concentration of oxidizable Fe(II) species in seawater solutions containing added iron. Although this technique had the advantage of allowing "in situ" analysis without undue perturbation of the system, sensitivity was still insufficient to detect any production of $\mathrm{Fe}$ (II) upon irradiation of a seawater sample at natural $\mathrm{pH}$.

Several systems for the detection of Fe(II) have since been developed, with sufficient sensitivity for the determination of $\mathrm{Fe}$ (II) in seawater at ambient $\mathrm{pH}$ and naturallyoccurring Fe concentrations, even in open ocean environments. King et. al., 1991 published details of a method involving preconcentration of Fe(II) on a C-18 Sep-Pak cartridge impregnated with the strong Fe(II)-specific chelator ferrozine. This method, reported to have a detection limit of $0.12 \mathrm{nmol} / \mathrm{kg}$, was employed by O'Sullivan et. al., 1991 in a study of $\mathrm{Fe}$ (II) in the surface waters of the equatorial Pacific. The results of O'Sullivan et. al. indicated surprisingly high levels of Fe(II) in equatorial Pacific waters, 
generally higher in surface waters than total dissolved iron concentrations determined independently by Martin et. al., 1991. The validity of the Fe(II) results is therefore suspect.

A number of techniques have subsequently been introduced that rely on flow injection with chemiluminescence detection for the determination of various forms of iron (Elrod et. al., 1991; Obata et. al. 1993; King et. al., 1995; O’Sullivan et. al., 1991; Measures et. al., 1995). Some of these techniques detect Fe(II) specifically, and have been employed in laboratory studies of Fe redox reactions in seawater (eg. King et. al., 1995; Voelker and Sedlak 1995). In some cases, techniques based on the chemiluminescent detection of $\mathrm{Fe}$ (II) have been modified by the addition of $\mathrm{pH}$ buffers and/or reducing agents to provide a measure of the amount of reducible iron in seawater (O'Sullivan et. al., 1995; Johnson et. al., 1994). This is an operational measurement that corresponds to iron species in seawater labile enough to be reduced and detected under the measurement conditions used. This pool of iron can include dissolved Fe(II) and Fe(III) species, as well as amorphous iron oxide solids. Measurement of reducible iron has been used in studies of iron oxide photochemical reactions in seawater at ambient (nM) concentrations in the equatorial Pacific (Johnson et. al., 1994).

A method that is conceptually similar to the measurement of reductively-labile iron is the oxine lability technique of Wells et. al., 1991a. Rather than reduction of iron, this technique is based on the principle of competitive ligand exchange. An excess of oxine, a strong iron(III)-binding ligand, is added to a seawater sample buffered at $\mathrm{pH} 6$. After incubation under these conditions for one hour, the iron bound to oxine is extracted from the seawater onto a C-18 Sep-Pak cartridge, and eluted from the cartridge with methanol. This method has been used in studies of the photoreduction of iron oxides in seawater (Wells et. al., 1991b; Wells and Mayer, 1991a) and in studies of iron bioavailability (Wells et. al., 1991a; Wells and Mayer, 1991b). Like the reducible iron methods, oxine lability is an operationally-defined measurement that detects dissolved inorganic or weakly complexed forms of iron, as well as amorphous iron oxides. 
Several additional competitive ligand exchange techniques have been developed for the analysis of iron in seawater, based on the addition of iron-complexing ligands and detection of specific iron complexes via cathodic stripping voltammetry (CSV) (Gledhill and van den Berg, 1995; Wu and Luther, 1995; Rue and Bruland, 1995). These methods have the advantage of high sensitivity and minimal sample treatment. Competitive ligand exchange/CSV techniques have generally been used in combination with titration methods in order to determine the concentration and binding constants of natural $\mathrm{Fe}$ (III)binding ligands, although Wu and Luther, 1995 have in addition employed a kinetic approach to study the complexation of $\mathrm{Fe}$ (III) by natural ligands. Competitive ligand exchange with CSV detection can also be used to study changes in iron oxide reactivity due to thermal maturation and/or reductive processes (Wu and Luther, 1995).

\section{Novel method for studying reactions of synthetic iron oxides in seawater}

The techniques described above that have been used to study reactions of iron oxides in seawater all rely on the detection of reactive intermediate iron species. In the case of methods specific for $\mathrm{Fe}(\mathrm{II})$, this is a reactive intermediate with a half-life on the order of a few minutes in oxygenated seawater at $\mathrm{pH} 8$ (O'Sullivan et. al., 1991); in the case of methods specific for iron species labile to reduction and/or competitive ligand exchange, half-lives with respect to hydrolysis and polymerization reactions may be in the range of 10's of hours (Wells and Mayer, 1991a; Wu and Luther, 1995; Johnson et. al., 1994). In order to study iron oxide reactions using any of these methods, therefore, it is necessary that the rate of formation of reactive iron species (as determined by the analytical method employed) exceeds the removal rate of those species to the degree that a detectable surplus or steady-state level of reactive iron develops. Processes that account for the removal of reactive iron species can include not only the oxidation and hydrolysis/polymerization reactions mentioned above, but also the biological uptake and sequestration of available iron, complexation of iron by strong binding ligands, and the aggregation of colloidal or particulate iron. 
Analytical techniques involving the detection of reactive iron, therefore, are ill-suited to the study of processes that dissolve iron oxides or modify oxide reactivity with relatively low specific rate constants, or that involve sequestration of reactive iron due to biological uptake, strong complexation, etc. This chapter describes the development and application of a technique that is well-suited to study reactions of iron oxide via these types of processes. Rather than focusing on reactive iron, this method involves:

1) Synthesis of synthetic iron oxides uniformly impregnated with an inert tracer; 2) Detection of tracer released to the dissolved phase upon dissolution or modification of the oxide structure due to reactive processes. Once released from the iron oxide matrix, the tracer remains stable in the dissolved phase due to a lack of particle reactivity, involvement in biological cycling, or other removal processes.

Thus, over time and with continued reaction of the synthetic oxide, the inert tracer gradually accumulates in the dissolved phase, providing an "integrated" signal of iron oxide reaction. In this way, even though the specific reaction rate of the iron oxide may be low enough that measurable amounts of reactive iron do not accumulate in the system, release of the inert tracer from the oxide matrix and its accumulation in the dissolved phase provide a signal of the extent of reaction undergone by the iron oxide. In addition to its use in studies of iron oxide reactions that proceed relatively slowly, this technique also provides an independent, complementary means to study faster processes, such as photoreduction, that have already been examined using techniques like oxine lability (Wells and Mayer, 1991a) or total reducible iron (Johnson et. al., 1994).

\section{Method Development - Chapter Overview}

The following experimental section begins with a description of the initial attempt to develop the technique described above, based on the synthesis of colloidal ferrihydrite impregnated with ${ }^{51} \mathrm{Cr}$ as $\mathrm{Cr}(\mathrm{VI})$. Colloid synthesis, initial testing and experimental applications are described, leading to the result that $\mathrm{Cr}(\mathrm{VI})$ is inappropriate as an inert tracer. The rest of the experimental section details the subsequent, successful attempt to use ${ }^{133} \mathrm{Ba}$ as the inert tracer. Synthesis of several types of ${ }^{133} \mathrm{Ba}$-impregnated iron oxides 
are described. A description follows of the testing and initial experimental application of ${ }^{133} \mathrm{Ba}$-impregnated ferrihydrite colloids rinsed via dialysis. ${ }^{133} \mathrm{Ba}$-impregnated ferrihydrite colloids rinsed via successive filtration and resuspension are also discussed. The chapter concludes with a discussion of the mechanistics of the method, comparison to other techniques, and prospects for future use.

\section{Experimental}

\section{Initial attempt $-{ }^{51} \mathrm{Cr}(\mathrm{VI})$}

Choice of tracer - In the first attempt to develop an inert tracer methodology for following the reaction of iron oxides in seawater, ${ }^{51} \mathrm{Cr}$ (VI) was chosen as the inert tracer. This choice was based upon the following three criteria: 1) Uniform incorporation into the iron oxide matrix during synthesis: Ferrihydrite, the iron oxide used in our previous work as a model iron oxide phase (see Chapter 3), was synthesized by hydrolysis of acidic Fe(III) solution (Wells et. al., 1991a). At the synthesis $\mathrm{pH}$ near 3, $\mathrm{Cr}$ (VI) occurs as an anion, $\mathrm{HCrO}_{4}^{-}$, and thus should be efficiently co-precipitated with the positivelycharged iron oxide phase (Dzombak and Morel, 1990).

2) Efficient release from the iron oxide phase in seawater; no scavenging by other solids or biological uptake: In seawater, with the iron oxide surface neutral to slightly positively-charged, the $\mathrm{CrO}_{4}{ }^{2-}$ anion should have little to no affinity for the oxide surface and thus be efficiently released (Dzombak and Morel, 1990). The high concentrations of $\mathrm{SO}_{4}{ }^{2-}$ in seawater should also contribute to release of the tracer via ion exchange. Most divalent anions in seawater (barring phosphate) are generally non-particle and nonbiologically reactive (Bruland, 1983), so uptake by phases other than the iron oxide itself should not be a problem. 3) Tracer should be capable of sensitive detection, with a minimum of sample prep: ${ }^{51} \mathrm{Cr}$ has a gamma emission which is detected with reasonable sensitivity by our Canberra low-energy germanium detector. 
Colloid synthesis - Colloids were synthesized essentially according to the same protocol described in Chapter 3, taken from Wells et. al., 1991a, i.e. hydrolysis of a $400 \mu \mathrm{M}$ solution of $\mathrm{FeCl}_{3}$ at $\mathrm{pH} \sim 3.3$ for $<1 \mathrm{~h}$, followed by a brief heating phase. Colloids were co-labeled with ${ }^{59} \mathrm{Fe}$ (III) and ${ }^{51} \mathrm{Cr}(\mathrm{VI})$. Both isotopes were added to the solution just prior to dissolving the ferric chloride. For these colloids, generally a heating step of 5-10 minutes at $90^{\circ} \mathrm{C}$ was used. These colloids were also extracted with ionic exhange resin by the slurry method as described in Chapter 3, but with a slight modification. In order to remove excess ${ }^{51} \mathrm{Cr}$ (as $\mathrm{HCrO}_{4}^{-}$at $\mathrm{pH} 3$ ) as well as excess reactive $\mathrm{Fe}$, colloids were extracted (15 min.) in a slurry of both cation and anion exchange resin, added at a ratio of $2 \mathrm{x}$ (by weight) anion exchange resin (Biorad 100-200 mesh AG 1-X8, $\mathrm{Cl}^{-}$form) to cation exchange resin (Biorad 100-200 mesh AG 50W-X8, $\mathrm{H}^{+}$form). Colloids and resin beads were separated by filtration through a glass frit, as described previously.

Co-dissolution - In order to use production of dissolved ${ }^{51} \mathrm{Cr}$ in seawater as a proxy for the dissolution of the iron oxide phase, it was necessary to demonstrate co-dissolution of $\mathrm{Fe}$ and ${ }^{51} \mathrm{Cr}$. In other words, as the colloidal ferrihydrite dissolves, soluble ${ }^{51} \mathrm{Cr}$ should be produced in a constant ratio to soluble $\mathrm{Fe}$. This indicates that the ${ }^{51} \mathrm{Cr}$ is incorporated uniformly throughout the Fe oxide matrix, not preferentially concentrated near the oxide surface, or within the inner regions of the colloidal sub-units (Cornell and Schwertmann, 1996). In order to demonstrate co-dissolution, an extended resin extraction was performed on a batch of colloids labeled with both ${ }^{59} \mathrm{Fe}$ and ${ }^{51} \mathrm{Cr}$. The extraction protocol was similar to that described above, but extended over a much longer time period in order to completely dissolve the colloids.

Starting at $t_{0}$, the colloidal sol was sampled to determine the total concentration of ${ }^{51} \mathrm{Cr}$ and ${ }^{59} \mathrm{Fe}$ prior to extraction. Then cation and anion exchange resin were added and stirred to create a slurry. Periodically aliquots of the slurry were removed and filtered through a coarse frit to remove the resin beads, and the aliquot of extracted sol saved for radioactive counting. In this way, the disappearance of each isotope from the colloidal sol was monitored. Results are shown in Figure 4.1A in terms of relative $\%$ of ${ }^{51} \mathrm{Cr}$ and ${ }^{59} \mathrm{Fe}$ (vs 

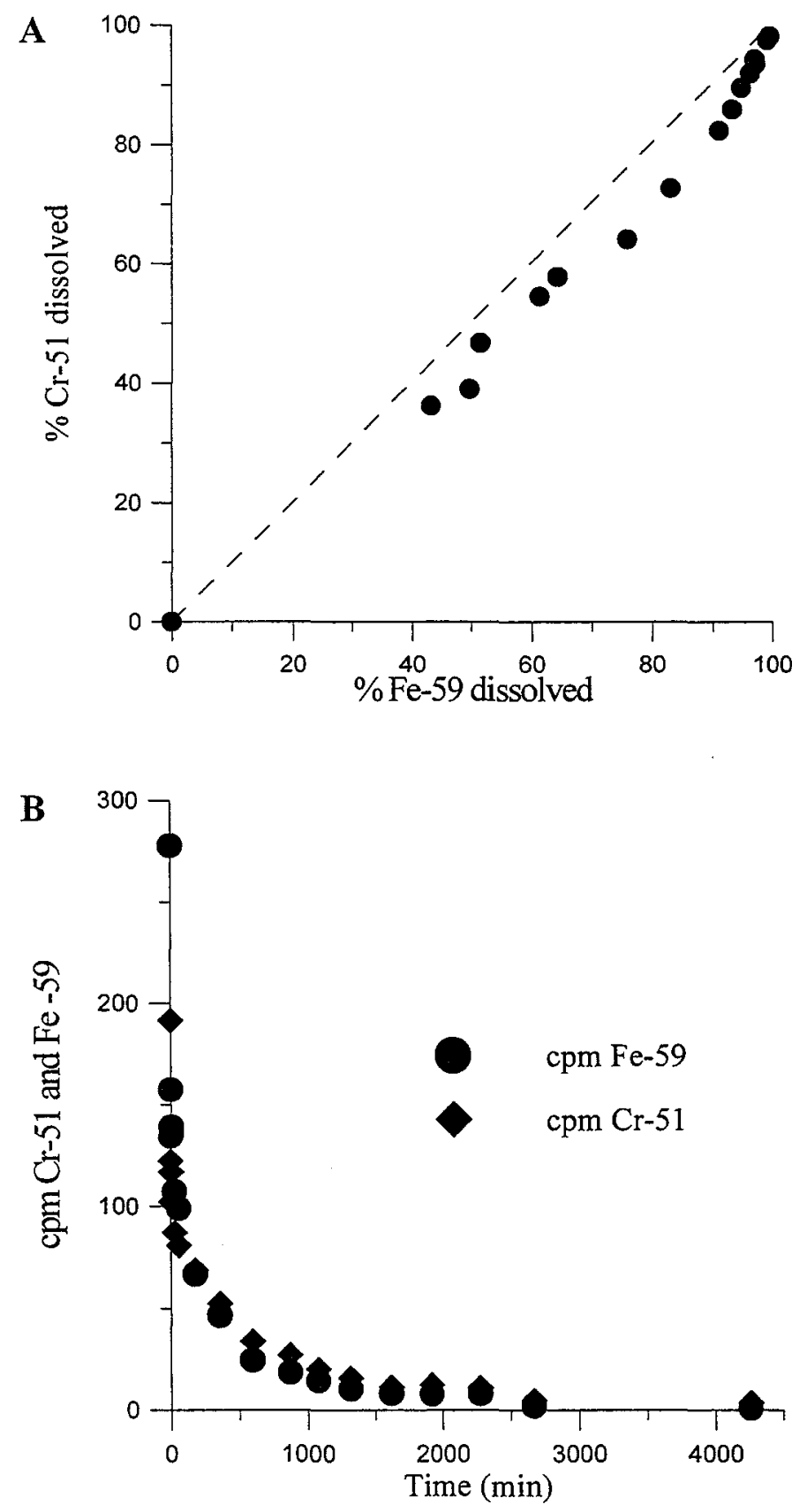

Figure 4.1 - Resin co-dissolution curves for ferrihydrite impregnated with 51-Cr

A. $\% \mathrm{Cr}-51$ vs $\% \mathrm{Fe}-59$ removed from colloids

B. CPM Cr-51 and Fe-59 remaining in sol vs time 
the ideal co-dissolution line), and in $4.1 \mathrm{~B}$ in terms of $\mathrm{cpm}^{51} \mathrm{Cr}$ and ${ }^{59} \mathrm{Fe}$ vs time. It is apparent that the two isotopes track each other quite well, showing co-dissolution of ${ }^{51} \mathrm{Cr}$ and $\mathrm{Fe}$ (as ${ }^{59} \mathrm{Fe}$ ) as the structure of the $\mathrm{Fe}$ oxide is broken down due to interaction with the cation exchange resin. This indicates that ${ }^{51} \mathrm{Cr}$ is distributed uniformly throughout the Fe oxide matrix, so the production of dissolved ${ }^{51} \mathrm{Cr}$ upon reaction of the colloidal ferrihydrite in seawater can be taken as a proxy for the extent of reaction of the Fe oxide phase.

Photochemical Experiments - As an initial test of the ${ }^{51} \mathrm{Cr}$-impregnated Fe colloids, some simple photochemical experiments were conducted on May 2nd and 5th, 1996.

Ferrihydrite colloids dual-labeled with ${ }^{59} \mathrm{Fe}$ and ${ }^{51} \mathrm{Cr}$ were synthesized as described above and added at $\mu \mathrm{M}$ Fe concentrations to $0.2 \mu \mathrm{m}$-filtered Vineyard Sound water in duplicate $300 \mathrm{ml}$ quartz flasks. The flasks were incubated in natural sunlight outside the Fye Laboratory at WHOI, with one flask exposed to the sun and one flask shielded with aluminum foil as a dark control. No attempt was made to regulate the temperature; being early May the air was fairly cool. At $t_{0}$ and at intervals throughout the time course of irradiation, flasks were sampled for ${ }^{51} \mathrm{Cr}$ and ${ }^{59} \mathrm{Fe}$ activity in total and $0.05 \mu \mathrm{m}$-filtered aliquots. Results for the two experiments are shown in Figure 4.2 as \% of total ${ }^{51} \mathrm{Cr}$ in $0.05 \mu \mathrm{m}$ filtrates vs time in both the lighted flasks and dark controls. In both experiments, there was a significant increase in dissolved ${ }^{51} \mathrm{Cr}$ in the lighted flasks relative to the dark controls. No ${ }^{59} \mathrm{Fe}$ activity was detected in the filtrates for either experiment. These results appear to confirm the validity of the method, at least for photochemical measurements. As a result of photoreductive reactions occuring at the iron oxide surface, ${ }^{51} \mathrm{Cr}(\mathrm{VI})$ was released from the iron oxide matrix into the dissolved phase. $\mathrm{Fe}$ (as ${ }^{59} \mathrm{Fe}$ ) was rapidly scavenged back onto the oxide surface, showing no increase in the dissolved phase.

Grazing Experiments - On a cruise to the Sargasso Sea in June, $1996,{ }^{51} \mathrm{Cr}$-impregnated ferrihydrite colloids were employed in a variety of on-deck incubation studies of protozoan-mediated colloidal Fe oxide dissolution. Results were all negative, showing no 

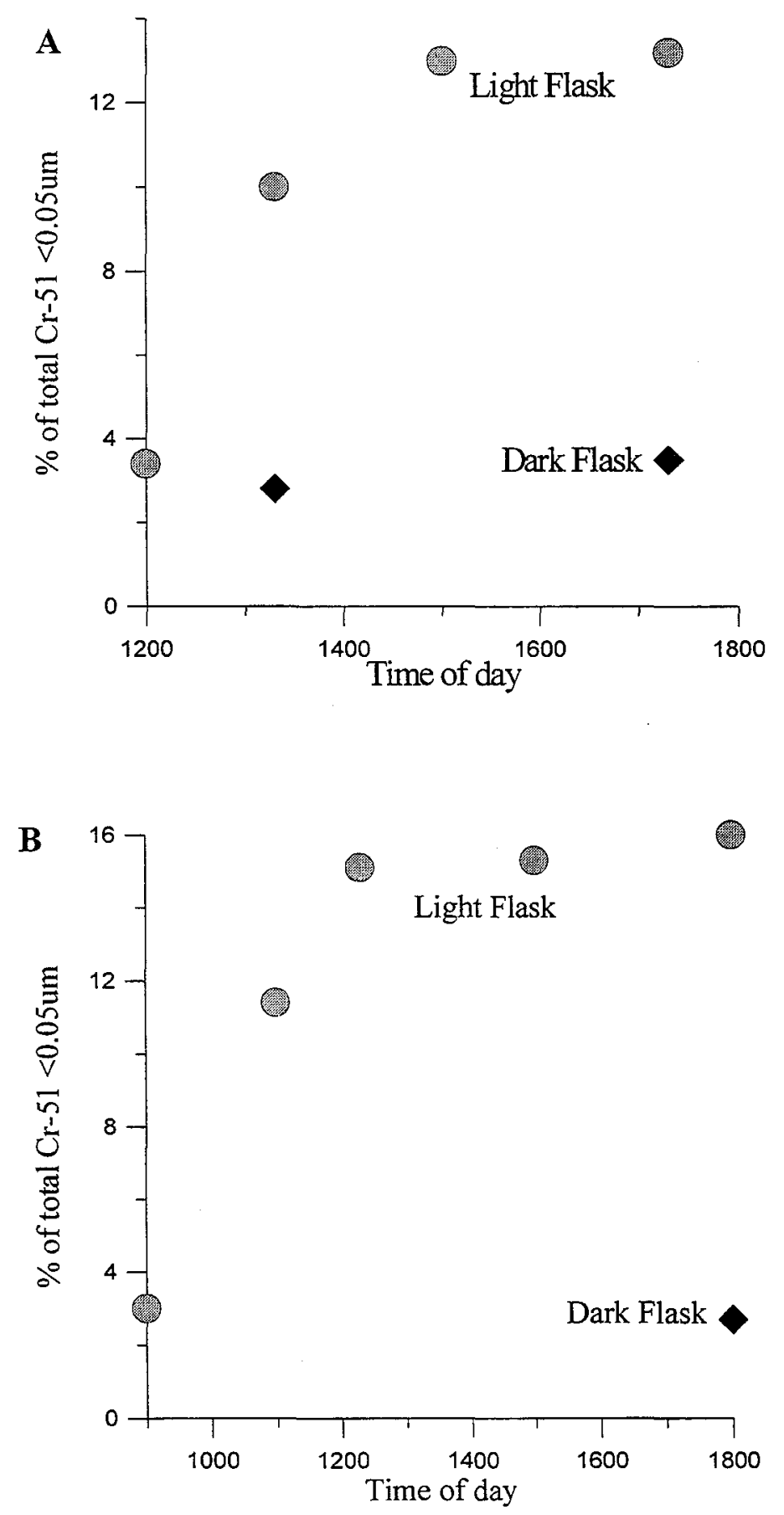

Figure 4.2 - Photochemical experiments with Cr-51-impregnated ferrihydrite in natural sunlight. Dissolved $\mathrm{Cr}-51$ vs time in irradiated flasks and dark controls.

A. Photochemical experiment \#1,5/2

B. Photochemical experiment $\# 2,5 / 5$ 
production of dissolved ${ }^{51} \mathrm{Cr}$ in incubations with active grazers relative to controls. After this cruise, a grazing experiment was conducted in the laboratory with ${ }^{51} \mathrm{Cr}$-impregnated Fe oxides, using the microflagellate Cafeteria as the grazer. This organism had been shown repeatedly in previous work (see Chapter 3 ) to be capable of dissolving colloidal ferrihydrite, yet there was no increase in filterable ${ }^{51} \mathrm{Cr}$ in the grazing cultures in this experiment relative to controls, despite consumption of bacterial biomass as normal by the grazer. This indicated that the tracer was not operating as predicted in the culture system.

Conclusion - Subsequent to these results, Deng and Stone, 1996, published an article on the surface-catalyzed reduction of $\mathrm{Cr}(\mathrm{VI})$ by low molecular weight organic compounds. These authors found that the reduction of $\mathrm{Cr}(\mathrm{VI})$ by simple organic acids, esters, and phenols could be catalyzed by a variety of oxide surfaces, including goethite ( $\alpha-\mathrm{FeOOH})$. The catalytic effect is enhanced at low $\mathrm{pH}$, due to greater adsorption of $\mathrm{Cr}(\mathrm{VI})$ onto the Fe oxide. This mechanism could account for the failure of the ${ }^{51} \mathrm{Cr}$-impregnated ferrihydrite to demonstrate any release of dissolved ${ }^{51} \mathrm{Cr}$ despite grazer-mediated dissolution. In the acidic conditions and elevated concentrations of organic matter that occur within the protozoan food vacuole, $\mathrm{Cr}(\mathrm{VI})$ might be quite readily reduced to $\mathrm{Cr}$ (III) via the catalytic mechanism proposed by Deng and Stone. $\mathrm{Cr}(\mathrm{III})$, a trivalent cation, has a chemistry similar in many respects to Fe(III). It would likely behave in a manner similar to Fe, i.e. re-precipitating back onto oxide surfaces in seawater. This would account for the lack of dissolved ${ }^{51} \mathrm{Cr}$ observed in this experiment.

${ }^{51} \mathrm{Cr}$ (VI) thus appears to be inappropriate as an inert tracer for iron oxide reactions, due to its redox chemistry. The potential for reduction of $\mathrm{Cr}(\mathrm{VI})$ at the iron oxide surface would complicate any attempt to utilize release of dissolved ${ }^{51} \mathrm{Cr}$ as an indicator of the extent of reaction. Nonetheless it is worth noting that the photochemical experiments in Figure 4.2 did indicate substantial release of dissolved ${ }^{51} \mathrm{Cr}$ as a consequence of photochemical reactions at the iron oxide surface. This may be due to the fact that the photochemical reactions took place in ambient seawater at $\mathrm{pH} 8$, thus decreasing the 
affinity of $\mathrm{Cr}(\mathrm{VI})$ for the $\mathrm{Fe}$ oxide surface and facilitating its release to the dissolved phase before oxide-catalyzed reduction could take place. The surfaces of Fe oxides during photochemical reactions in surface waters have been proposed to be a strongly oxidizing environment (Moffett and Zafiriou, 1993), which may keep Cr(VI) from being reduced. The protistan food vacuole, in contrast, is likely to be acidic and reducing, effectively trapping $\mathrm{Cr}$ (VI) at the oxide surface and facilitiating its reduction to $\mathrm{Cr}(\mathrm{III})$. Thus, the different behavior of the ${ }^{51} \mathrm{Cr}$-impregnated ferrihydrite in the case of photochemical dissolution vs. protozoan-mediated dissolution may indicate fundamental differences in the nature of these processes, and in their effect on the surface chemistry of iron oxides.

\section{$\underline{\text { Second attempt }-{ }^{133} \mathrm{Ba}}$}

Choice of tracer - The preliminary results with ${ }^{51} \mathrm{Cr}(\mathrm{VI})$ served to demonstrate that the inert tracer idea does work, in principle, as a means of following the reaction of iron oxides in seawater. Colloidal ferrihydrite was successfully synthesized with a tracer incorporated throughout the oxide matrix, and in photochemical experiments in seawater reaction of the iron oxide resulted in release of the tracer into the dissolved phase. Therefore a second attempt was made to develop the method, using a tracer that avoided the problems associated with ${ }^{51} \mathrm{Cr}(\mathrm{VI})$.

${ }^{133} \mathrm{Ba}$ was eventually chosen as the tracer, based on the following modified criteria: 1) No complicated redox chemistry: $\mathrm{Ba}$ is an alkali earth metal, and as such has only one oxidation state, +2 . Thus there is no chance of redox chemistry. 2) Uniform incorporation into the iron oxide matrix during synthesis: This necessitated a change in synthesis protocol, but incorporation is possible (see below). 3) Efficient release from the iron oxide phase in seawater; no scavenging by other solids or biological uptake: As a divalent cation with a relatively large ionic radius, $\mathrm{Ba}$ exhibits a very low partition coefficient for a variety of solids in seawater, including surface sediments (Nyffeler et. al., 1984), $\alpha-\mathrm{FeOOH}$, and montmorillonite (Li et. al., 1984). Based on its first hydrolysis constant, $\mathrm{Ba}$ is expected to have a very low surface complexation constant for binding to 
Fe oxides (Dzombak and Morel, 1990). The high concentrations of $\mathrm{Mg}^{2+}$ and $\mathrm{Ca}^{2+}$ in seawater should also help to buffer against scavenging of $\mathrm{Ba}$ by iron oxides or other particles. $\mathrm{Ba}$ is known to be involved in biological cycling, which results in its depletion in surface waters. This is thought to be due to the formation of barite, $\mathrm{BaSO}_{4}$, within microenvironments inside aggregates of decaying phytoplankton (Bishop, 1988; Dymond et. al., 1992). This process, although not well-characterized, is unlikely to occur to a significant extent on the timescale of the intended experiments ( 5 days at the most). In addition, the fairly high concentration of stable $\mathrm{Ba}$ in seawater should buffer against uptake of ${ }^{133} \mathrm{Ba}$ as barite. 4) Tracer should be capable of sensitive detection, with a minimum of sample prep: ${ }^{133} \mathrm{Ba}$ has an intense $\mathrm{X}$-ray emission at the very low end of the gamma spectrum (31.3 keV), for which the low-energy germanium detector in the Moffett laboratory is quite sensitive.

Colloid Synthesis - The very low affinity of $\mathrm{Ba}$ for iron oxide surfaces makes incorporating ${ }^{133} \mathrm{Ba}$ into ferrihydrite via co-precipitation somewhat challenging. It is not possible to co-precipitate $\mathrm{Ba}^{2+}$ during hydrolysis of acidic Fe(III) solution, as described previously for $\mathrm{Cr}(\mathrm{VI})$. In order to incorporate a measurable amount of ${ }^{133} \mathrm{Ba}$, synthesis of the ferrihydrite has to be carried out at a higher $\mathrm{pH}$ by rapidly neutralizing a concentrated ferric salt solution in the presence of a large excess of ${ }^{133} \mathrm{Ba}$. In this way, a small percentage of the ${ }^{133} \mathrm{Ba}$ is incorporated by the iron oxide phase.

The procedure for synthesizing ferrihydrite via neutralization of a ferric salt solution was derived from Eggleton and Fitzpatrick, (1988) and from Schwertmann and Cornell, (1991). To begin, ${ }^{133} \mathrm{Ba}$ ( $\mu \mathrm{M}$ concentration) and ${ }^{59} \mathrm{Fe}$ (trace amount) are added to Milli-Q water, and the $\mathrm{pH}$ of the water adjusted to $\sim 5$ (with rapid stirring). $\mathrm{Fe}\left(\mathrm{NO}_{3}\right)_{3} \cdot 9 \mathrm{H}_{2} \mathrm{O}$ is then added and dissolved (concentration $2.5 \mathrm{mM} \mathrm{Fe}$ ). A pre-calibrated amount of $0.0375 \mathrm{M} \mathrm{NaOH}$ solution (usually about $1 / 5$ the volume of the ferric nitrate solution) is then rapidly added to the ferric nitrate salt while stirring to quickly bring the $\mathrm{pH}$ to between 7 and 8 . This results in the instantaneous precipitation of orange flocs. The 
solution is allowed to stand in this condition for about 15 minutes prior to further processing.

The initial product of this basic hydrolysis is a 2-line ferrihydrite, the most amorphous form of the mineral. This material is, in general, too reactive for practical use in experiments. In order to decrease the reactivity of the 2-line ferrihydrite, it is heated at $90^{\circ} \mathrm{C}$ in a water bath for 5 minutes (at pH 7-8), and then rapidly cooled to room temperature by submersion in an ice-water bath. (For some experiments colloidal ferrihydrite heated at $70^{\circ} \mathrm{C}$ for 5 minutes was used; this phase demonstrated little difference in reactivity relative to $90^{\circ} \mathrm{C}$ ferrihydrite.) The heating step causes the phase to condense somewhat, so that it becomes more ordered but still sub-crystalline, more like a 6-line than a 2-line ferrihydrite.

This is the synthesis procedure for the ${ }^{133} \mathrm{Ba}$-impregnated colloidal ferrihydrite used in the experiments described in this chapter and the next. Using the same co-precipitation procedure as that described above, it is possible to make other iron oxide phases impregnated with ${ }^{133} \mathrm{Ba}$. This is because 2-line ferrihydrite is often a precursor for the synthesis of other iron oxides like goethite and hematite (Schwertmann and Cornell, 1991). Co-precipitation of other metals with ferrihydrite by neutralizing a mixed metal/Fe salt solution is a common first step in making metal-substituted goethite and hematite (Cornell and Schwertmann, 1996).

In order to make hematite impregnated with ${ }^{133} \mathrm{Ba}$, the original 2-line ferrihydrite can be heated at weakly alkaline $\mathrm{pH}$ at $90^{\circ} \mathrm{C}$ for a number of hours (see Schwertmann and Cornell, 1991 for specific details). This causes rearrangement and dehydration within ferrihydrite aggregates to form hematite. Because this transformation takes place under alkaline conditions, the ${ }^{133} \mathrm{Ba}$ should be retained by the iron oxide phase during the transformation. This has been tested by heating the 2-line ferrihydrite synthesized as described above at $90^{\circ} \mathrm{C}$ for periods of 10 minutes, 30 minutes, 2 hours, and 6 hours, at a $\mathrm{pH}$ of 7.8. The ${ }^{133} \mathrm{Ba}^{59} \mathrm{Fe}$ ratio of dialysis-rinsed products was about $2 \mathrm{x}$ as high in the 30 minute and 2 hour phases as in the 10 minute phase, probably due to better retention of ${ }^{133} \mathrm{Ba}$ by the more condensed phases during dialysis. The ${ }^{133} \mathrm{Ba} /{ }^{59} \mathrm{Fe}$ ratio decreased 
somewhat in the 6 hour phase, possibly due to expulsion of ${ }^{133} \mathrm{Ba}$ from the more crystalline, hematite-like structure. In appearance, the 6 hour phase had larger-sized subunits and the bright red color characteristic of hematite.

These results indicate that synthesis of ${ }^{133} \mathrm{Ba}$-impregnated hematite should be possible, although further work is needed to characterize the distribution of ${ }^{133} \mathrm{Ba}$ within the hematite crystal lattice. It sould also be possible to synthesize goethite impregnated with ${ }^{133} \mathrm{Ba}$ by precipitating ${ }^{133} \mathrm{Ba}$-impregnated 2-line ferrihydrite and subsequently holding the product under very basic conditions $(\mathrm{pH}>12)$ at $70^{\circ} \mathrm{C}$ for several days (Schwertmann and Cornell, 1991). Under these conditions, the ferrihydrite precipitate dissolves to form $\mathrm{Fe}(\mathrm{OH})_{4}{ }^{-}$species from which goethite nucleates and grows. Despite dissolution of the initial ferrihydrite phase, the ${ }^{133} \mathrm{Ba}$ would most likely remain in association with the forming goethite throughout this process, due to the very high $\mathrm{pH}$ of synthesis. This has been shown in the synthesis of $\mathrm{Ni}(\mathrm{II})-, \mathrm{Cu}(\mathrm{II})$ - and $\mathrm{Zn}$ (II)- substituted goethite (Cornell and Schwertmann, 1996).

A further iron oxide phase that can be synthesized with incorporated ${ }^{133} \mathrm{Ba}$ is lepidocrocite. Lepidocrocite is commonly synthesized by slow oxidation/hydrolysis of Fe(II) solutions buffered at pH 5-7.5 (Schwertmann and Cornell, 1991). ${ }^{133} \mathrm{Ba}-$ impregnated lepidocrocite has been synthesized by following the procedure outlined by Waite and Morel, 1984a, for lepidocrocite synthesis in a mM solution of $\mathrm{Fe}(\mathrm{III})$ at $\mathrm{pH} 7.4$ using a $\mathrm{CO}_{2}$ /air mixture to buffer the reaction. Lepidocrocite was synthesized according to the Waite and Morel protocol in the presence of $5 \times 10^{-8} \mathrm{M}{ }^{133} \mathrm{Ba}$. The final product incorporated approximately $5-10 \%$ of the ${ }^{133} \mathrm{Ba}$ present during synthesis. Further testing is required to determine how the ${ }^{133} \mathrm{Ba}$ is distributed within the lepidocrocite structure.

In all of the syntheses described above, it is important to note that the ${ }^{133} \mathrm{Ba}$ is only a trace component in the iron oxide matrix. During synthesis of ${ }^{133} \mathrm{Ba}$-impregnated 2-line ferrihydrite, the $\mathrm{Fe} /{ }^{133} \mathrm{Ba}$ ratio in solution is $10^{3} / 1$. In the final iron oxide product, the ratio is about $10^{5} / 1$ due to the inefficiency with which $\mathrm{Ba}$ is incorporated into the iron oxide, even when co-precipitated under basic conditions in a concentrated iron solution. Thus the ${ }^{133} \mathrm{Ba}$ is not present in the oxide as a solid solution or separate phase, but rather 
as a trace component scattered throughout the oxide matrix (see co-dissolution results below). As such, the ${ }^{133} \mathrm{Ba}$ does not have any effect on the structural or chemical properties of the iron oxide. Such effects are only known to occur at much higher levels of cation substitution (Cornell and Schwertmann, 1996). The sensitivity of the method can be increased by increasing the concentration of ${ }^{133} \mathrm{Ba}$ present during the coprcipitation step. In this way, it should be possible to incorporate up to $10 \mathrm{x}$ more ${ }^{133} \mathrm{Ba}$ without affecting the Fe oxide structure.

The ${ }^{133} \mathrm{Ba}$-impregnated iron oxide used in the experiments described in this chapter is a 6-line ferrihydrite. Before this product can be used in tracer experiments, it is critical to thoroughly rinse away the large excess of ${ }^{133} \mathrm{Ba}$ that does not actually get incorporated into the oxide matrix during co-precipitation. Several methods of rinsing the colloidal ferrihydrite were investigated in this work, involving both dialysis and filtration/resuspension techniques. The type of rinsing method used has a significant effect on the properties of the ${ }^{133} \mathrm{Ba}$-impregnated iron oxide and on its use as a tracer. The following section will describe ${ }^{133} \mathrm{Ba}$-colloidal ferrihydrite rinsed via dialysis, including the dialysis protocol, co-dissolution results, and a number of experimental applications of these synthetic iron oxides in mechanistic studies. The final portion of the experimental section will discuss ${ }^{133} \mathrm{Ba}$-impregnated colloidal ferrihydrite rinsed via filtration/resuspension.

\section{${ }^{133}$ Ba-impregnated ferrihydrite - dialyzed colloids}

Dialysis protocol - Following the 5 minute heating period at $90^{\circ} \mathrm{C}$, the colloidal ferrihydrite co-precipitated with ${ }^{133} \mathrm{Ba}$ is rapidly cooled to room temperature. The phase is allowed to equilibrate for a few minutes, then the suspension is well-mixed and diluted by a factor of 10 with Milli-Q water. The $\mathrm{pH}$ is adjusted to 5 by adding dilute $\mathrm{HCl}$. The solution is then briefly ultrasonicated with a $1 / 8$ " probe in order to disperse the aggregated colloidal suspension, forming a stable colloidal sol at $\mathrm{pH}$. The colloidal sol is loaded into dialysis bags (Spectrum, Spectra/Por CE Sterile Membranes, 3500 MWCO, $20 \mathrm{ml}$ sample volume). The colloids are dialyzed in Milli-Q water at $\mathrm{pH} 5$ for 60 hours, 
changing the dialysis water 5 times during that period. Dialysis containers are maintained open under a laminar flow hood, with constant stirring. Up to $60 \mathrm{ml}$ of colloidal sol (i.e. 3 dialysis bags) can be dialyzed at once in a 1 liter polycarbonate container. Generally, $10^{-4} \mathrm{M}$ stable $\mathrm{BaCl}_{2}$ is added to the $3 \mathrm{rd}$ or 4 th batch of dialysis water in order to enhance removal of ${ }^{133} \mathrm{Ba}$ from the surface of the iron oxide. If the colloidal sol de-stabilizes during the dialysis period (indicated by the settling of a small amount of orange precipitate at the bottom of the dialysis bags), it is quantitatively removed from the dialysis bags, re-sonicated, and put back into the bags for further dialysis. Colloids are never re-sonicated less than 20 hours before use in an experiment.

Counting ${ }^{59} \mathrm{Fe}$ and ${ }^{133} \mathrm{Ba}$ activities of co-labeled colloidal ferrihydrite both before and after the dialysis procedure indicate that, in general, dialysis removes about $20-30 \%$ of the ${ }^{59} \mathrm{Fe}$ and $96-99 \%$ of the ${ }^{133} \mathrm{Ba}$. Loss of the iron may be due to partial dissolution of the ferrihydrite at $\mathrm{pH} 5$, and/or scavenging of colloids onto the surface of the dialysis bags. The dialysis procedure results in a colloidal ferrihydrite that can be added to seawater at $\mu \mathrm{M}$ concentrations and preserve a low ${ }^{133} \mathrm{Ba}$ blank ( $~ 5 \%$ or less) under nonreactive conditions for extended periods of time (several days). These colloids were used in experiments with protozoan grazers, and also photochemical and thermal dissolution experiments. The colloids are fairly refractory for ferrihydrite, as indicated by the low ${ }^{133} \mathrm{Ba}$ blank and by measurements of oxine lability that indicate $2-3 \%$ of the iron to be oxine-labile (see Chapter 3 for oxine protocol). This level of oxine reactivity is similar to that of the colloidal ferrihydrite used in grazing experiments in Chapter 3.

As with the colloidal ferrihydrite described in Chapter 3, the ${ }^{133} \mathrm{Ba}$-impregnated ferrihydrite could vary somewhat in reactivity from batch to batch due to small variations in the synthesis protocol. Thus, colloids were synthesized fresh for each experiment, and reactivity in experimental systems is always normalized to reactivity in controls.

Co-dissolution - As explained for the ${ }^{51} \mathrm{Cr}(\mathrm{VI})$-impregnated colloidal ferrihydrite, it was necessary to demonstrate co-dissolution of ${ }^{133} \mathrm{Ba}$ and $\mathrm{Fe}$ for the ${ }^{133} \mathrm{Ba}$-impregnated colloidal ferrihydrite. A series of co-dissolution experiments was performed, using 
ferrihydrite co-labeled with ${ }^{59} \mathrm{Fe}$ and ${ }^{133} \mathrm{Ba}$ and rinsed by dialysis as described above. Figure 4.3 shows the results of a co-dissolution by resin extraction similar to the procedure described previously for the ${ }^{51} \mathrm{Cr}$-ferrihydrite. This extraction was performed with cation exchange resin only, at a pH of 5 . It is apparent that the ${ }^{133} \mathrm{Ba}$ and $\mathrm{Fe}$ (as ${ }^{59} \mathrm{Fe}$ ) dissolved congruently, demonstrating that ${ }^{133} \mathrm{Ba}$ is evenly distributed within the oxide framework. By 30 seconds, about $35 \%$ of the phase had already dissolved. In order to get a better idea of the coherence between ${ }^{133} \mathrm{Ba}$ and $\mathrm{Fe}$ at lower \% dissolution, additional co-dissolution experiments were performed.

Figure 4.4 shows a co-dissolution by citrate. For this experiment, dialysis-rinsed colloids were added to filtered Sargasso seawater at $\mu \mathrm{M}$ concentration, and allowed to equilibrate for 1 hour. At this point, $t_{0}$, a $0.05 \mu \mathrm{m}$ filtered aliquot and a total sample were taken. $\mathrm{pH}$ of the seawater was then reduced to 4 with $\mathrm{HCl}$, and citrate was added to a concentration of $100 \mu \mathrm{M}$. Subsequent filtered samples and totals were taken at 20 minutes, 1 hour, 3 hours, and 11.5 hours. It can be seen that ${ }^{133} \mathrm{Ba}$ and ${ }^{59} \mathrm{Fe}$ dissolved fairly congruently over the range of $0-30 \%$ dissolution. By the sample taken at 11.5 hours, wall loss had affected ${ }^{59} \mathrm{Fe}$, causing the point to fall somewhat off the 1:1 line. Wall loss of ${ }^{59} \mathrm{Fe}$ was about $11 \%$ by this time point, as indicated by the ${ }^{59} \mathrm{Fe}$ activity in total samples at $t_{11.5}$ relative to $t_{0}$.

In a similar experiment, Fe colloids were added to Sargasso seawater at $\mu \mathrm{M}$ concentration, equilibrated, and the $\mathrm{pH}$ of the seawater adjusted to 2.5. Over a time course of 4 hours, $0.05 \mu \mathrm{m}$ filtrate and total samples were taken. Dissolution was close to the 1:1 line (Fig. 4.5), although towards the latter part of the time course it appears that ${ }^{133} \mathrm{Ba}$ was being released from the colloids more efficiently than ${ }^{59} \mathrm{Fe}$. This is consistent with the greater affinity of $\mathrm{Fe}^{3+}$ relative to $\mathrm{Ba}^{2+}$ for the iron oxide surface at $\mathrm{pH} 2.5$.

Figure 4.6 shows a similar co-dissolution of ${ }^{133} \mathrm{Ba}$-ferrihydrite at $\mu \mathrm{M}$ concentrations in seawater which was acidified to about $\mathrm{pH} 2$ and to which hydroxylamine hydrochloride, a reducing agent, was added. This experiment also lasted 4 hours. Co-dissolution was again congruent, and the reductant apparently increased the rate of ${ }^{59} \mathrm{Fe}$ release from the oxide 


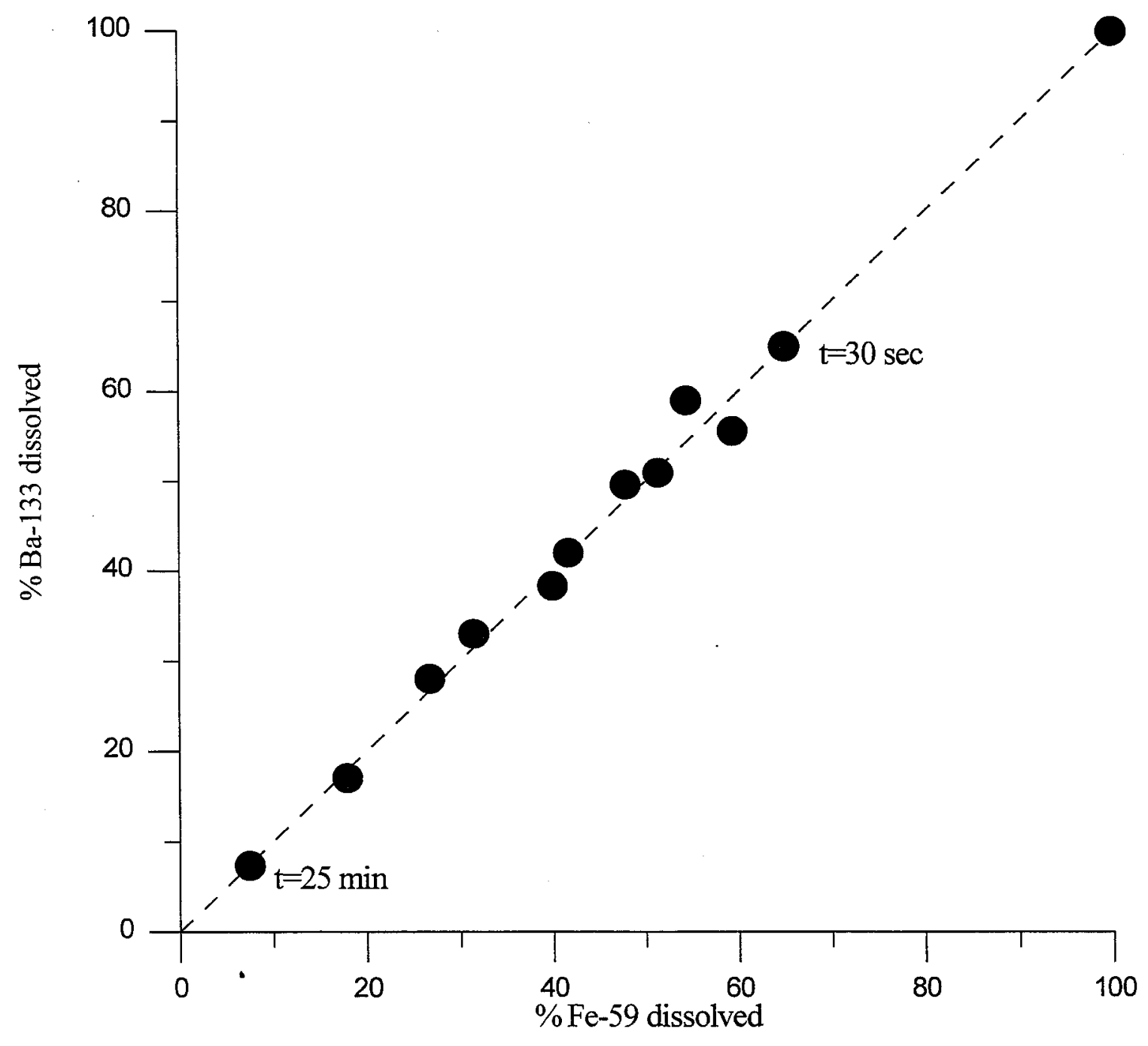

Figure 4.3 - Co-dissolution of $\mathrm{Ba}-133$ ferrihydrite via cation resin extraction. $\% \mathrm{Ba}-133$ vs $\% \mathrm{Fe}-59$ remaining in colloidal sol 


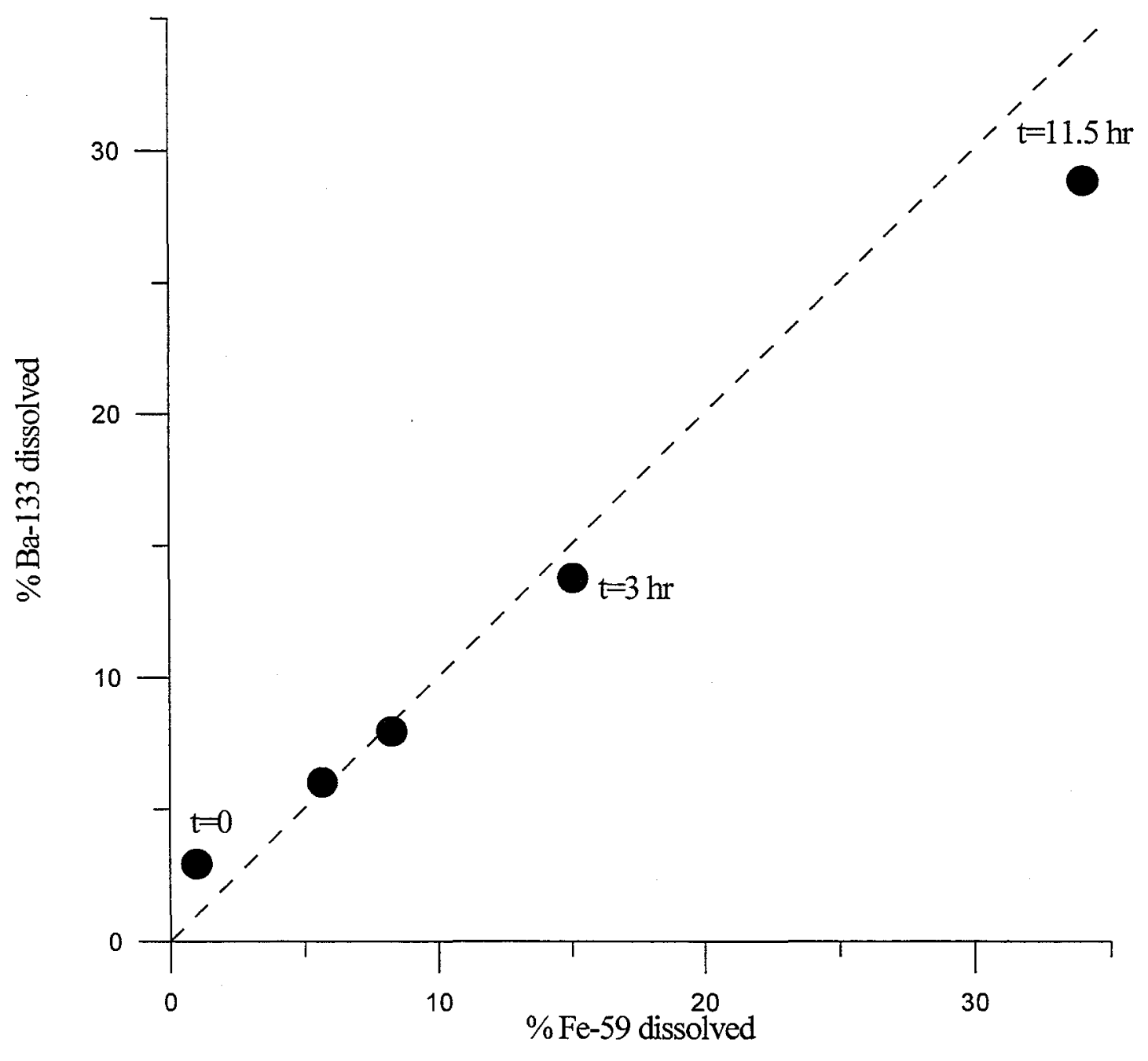

Figure 4.4 - Co-dissolution of Ba-133 ferrihydrite via citrate at $\mathrm{pH} 4$ in Sargasso seawater. \%Ba-133 dissolved vs $\% \mathrm{Fe}-59$ dissolved 


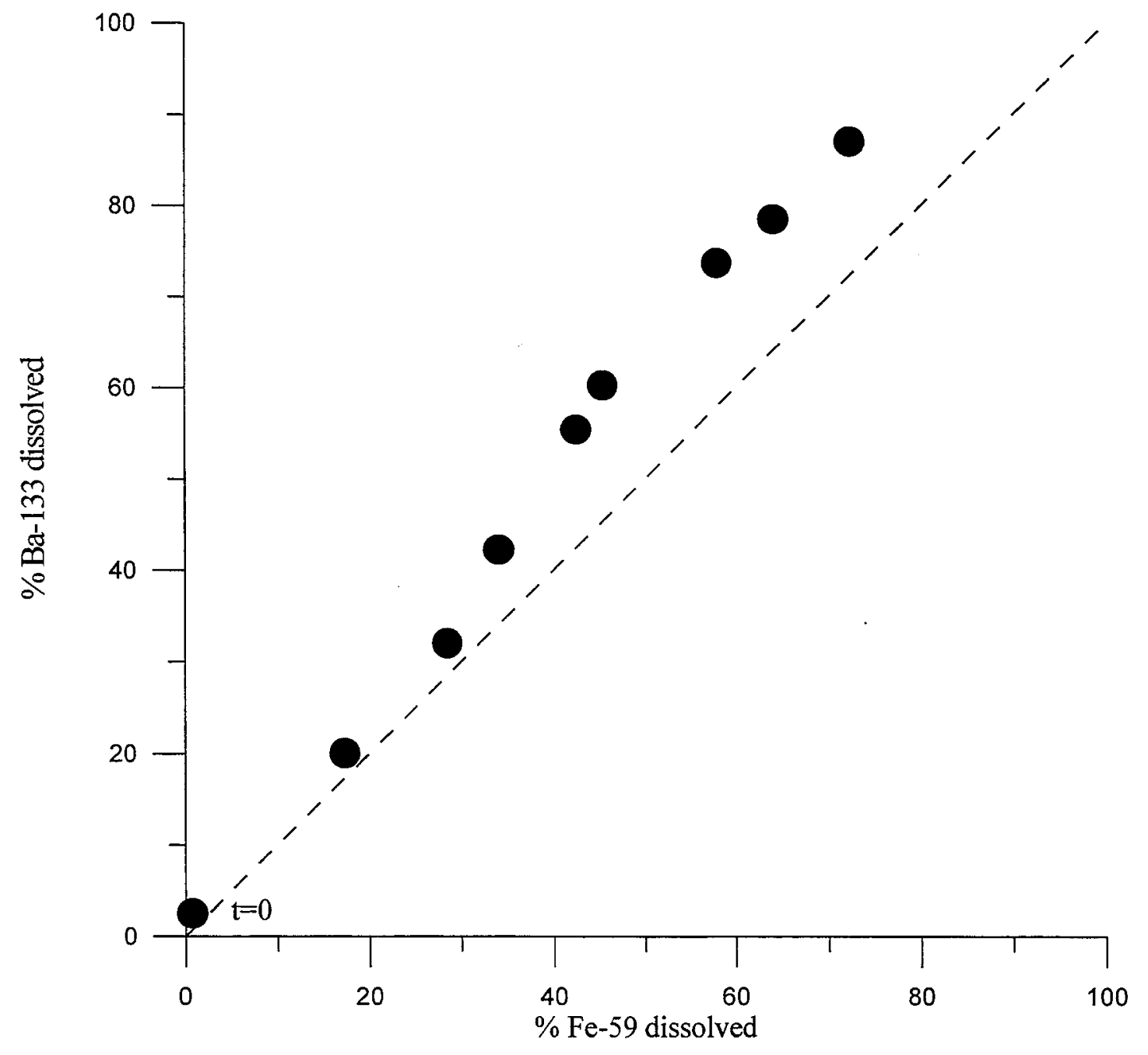

Figure 4.5 - Co-dissolution of Ba-133 ferrihydrite via acid, $\mathrm{pH}=2.5$ in Sargasso seawater. $\% \mathrm{Ba}-133$ vs $\% \mathrm{Fe}-59$ dissolved 


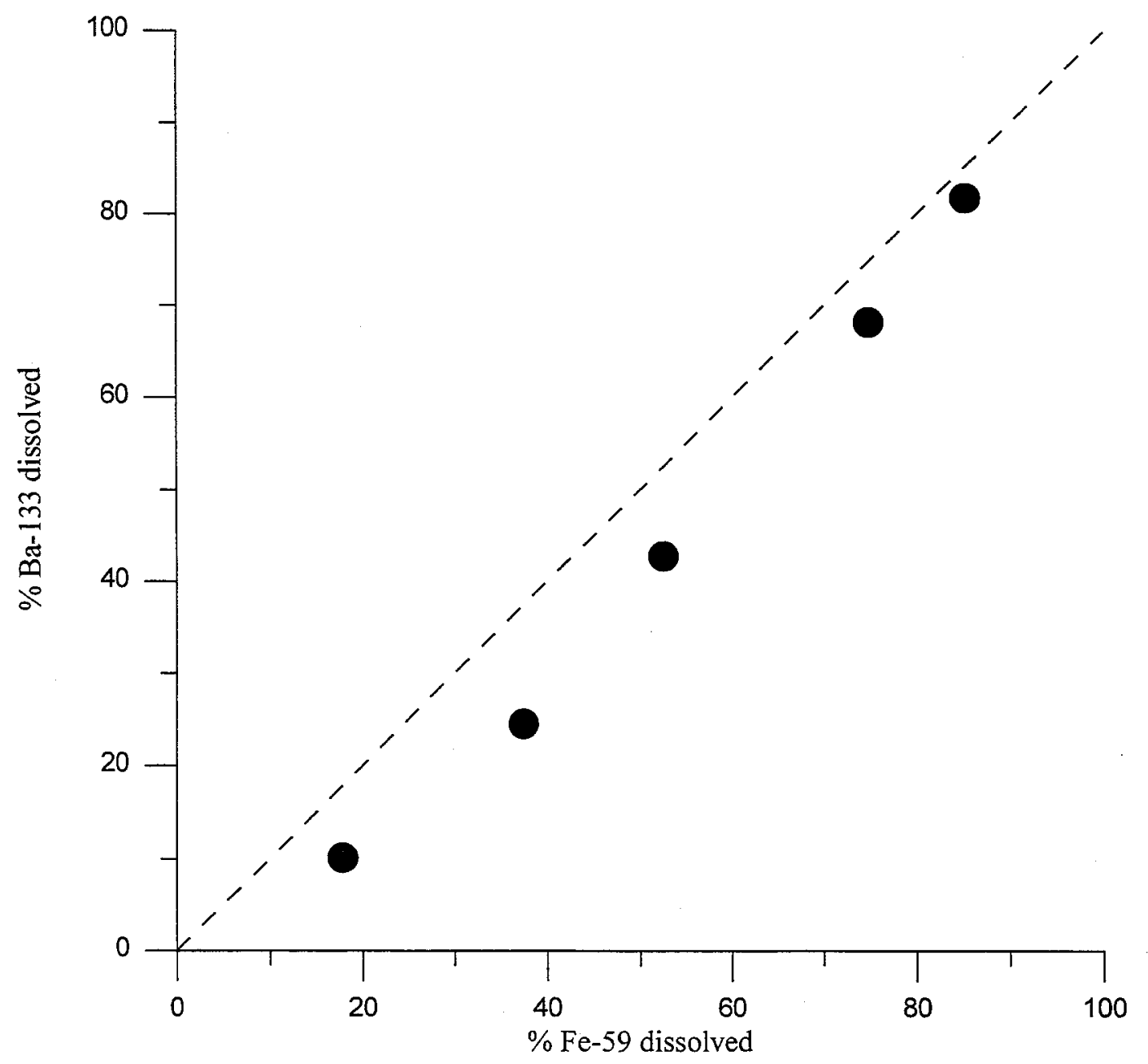

Figure 4.6 - Co-dissolution of Ba-133 ferrihydrite via hydroxylamine reduction in Sargasso seawater. \%Ba-133 vs \%Fe-59 dissolved 
surface. This is consistent with the fact that $\mathrm{Fe}^{2+}$ has a lower affinity for the oxide surface than $\mathrm{Fe}^{3+}$.

These data indicate that production of dissolved ${ }^{133} \mathrm{Ba}$ is a reasonable proxy for the dissolution of $\mathrm{Fe}$ oxides in seawater by a variety of mechanisms - reductive dissolution, complexation, and acidity. It should be noted, however, that these co-dissolution experiments were performed (of necessity) under conditions where ${ }^{59} \mathrm{Fe}$ released from the colloidal ferrihydrite would remain in the dissolved phase, so that the production of dissolved ${ }^{59} \mathrm{Fe}$ and dissolved ${ }^{133} \mathrm{Ba}$ could be compared. In subsequent experiments in this chapter and the next, the slow dissolution rate of the iron oxide and the chemical composition of the seawater $(\mathrm{pH} 8$; without high concentrations of complexing or reducing agents) are in most cases such that the fate of ${ }^{133} \mathrm{Ba}$ and $\mathrm{Fe}$ (stable $\mathrm{Fe}$ and ${ }^{59} \mathrm{Fe}$ ) is decoupled. While ${ }^{133} \mathrm{Ba}$, once released from the ferrihydrite matrix, accumulates in the dissolved phase, Fe readily re-precipitates, either back onto the surface of the oxide itself, or onto other available colloidal and particulate surfaces. (In some cases involving biota, the fate of Fe may be more complex, involving biological uptake as well as precipitation reactions.)

Under such circumstances, although dissolved ${ }^{133} \mathrm{Ba}$ is produced as a result of reactions undergone by ${ }^{133} \mathrm{Ba}$-impregnated ferrihydrite, it is not possible to say whether such reactions (and the concomitant accumulation of dissolved ${ }^{133} \mathrm{Ba}$ ) represent actual dissolution. ${ }^{133} \mathrm{~B}$ a release may also occur as a consequence of dissolution with subsequent reprecipitation, or even as a consequence of non-dissolution reactions involving some rearrangement of the iron oxide structure (eg. maturation reactions, ion exchange/sorption/desorption at the oxide surface, etc.). For this reason, the ${ }^{133} \mathrm{Ba}$ method is a tracer for the absolute rate and extent of iron oxide reaction (referring to a variety of possible reaction mechanisms) rather than just iron oxide dissolution.

It is apparent in Figs. 4.4-4.6, for example, that there is a small amount of ${ }^{133} \mathrm{Ba}$ in solution at $t_{0}$, even before dissolution of the ferrihydrite has begun by acidification or addition of reagents to the seawater. This ${ }^{133} \mathrm{Ba}$ "blank" is due to a variety of factors, including excess ${ }^{133} \mathrm{Ba}$ that may not have been rinsed away completely, and/or ${ }^{133} \mathrm{Ba}$ that 
may be released due to reactions at the Fe oxide surface (eg. ion exchange) when the ferrihydrite is diluted from the Milli-Q water dialysis matrix into seawater. Over longer timescales, maturation, sorption reactions, and thermal dissolution may contribute to the ${ }^{133} \mathrm{Ba}$ blank. Experiments employing ${ }^{133} \mathrm{Ba}$-impregnated ferrihydrite as a tracer for various types of $\mathrm{Fe}$ oxide reaction mechanisms must always include appropriate controls to correct for the blank, i.e. ${ }^{133} \mathrm{Ba}$ release that may occur due to mechanisms other than the one under study.

\section{Dialyzed colloids - Experimental applications}

The dialysis-rinsed colloids described above were used in a variety of experiments, looking at reactions of colloidal ferribydrite in seawater as mediated by photoreduction, ligand/thermal dissolution, and protozoan grazers. Photochemical and ligand/thermal results will be discussed here; experiments with protozoan grazers will be described in the following chapter.

Photochemical experiments provide a useful means of evaluating the performance of the inert tracer method as a technique for studying reactions of iron oxides in seawater. As described in the introduction to this chapter, the photochemistry of iron oxides in natural waters is an environmentally significant phenomenon and as such has been the object of much research. Previous studies provide a context within which our own results using a novel tracer methodology can be interpreted. Particularly useful for us with regard to reactions of iron oxides in seawater and under different conditions of $\mathrm{pH}$ and chromophore concentration is the work of Waite and Morel, 1984a and b; and Wells and Mayer 1991a.

Photochemical experiments with irradiation system - Protocols - A series of laboratory photochemical experiments was conducted in March 1997 using ${ }^{133}$ Ba-impregnated colloidal ferrihydrite. These experiments were done with the assistance of Phoebe Lam, an MIT undergraduate at the time, as part of her senior thesis project. The light source used in these experiments was a $1000 \mathrm{~W} \mathrm{Hg} / \mathrm{Xe}$ lamp equipped with an infrared filter 
and a pyrex ultraviolet filter. The lamp produced a well-collimated beam of light with a broad spectrum, about $300-800 \mathrm{~nm}$ in range. This approximates the solar spectrum as seen in surface waters. In terms of light intensity, this source is about ten times as intense as natural sunlight.

${ }^{133} \mathrm{Ba}$-impregnated colloidal ferrihydrite was synthesized as described above and colabeled with ${ }^{59} \mathrm{Fe}$. All of the colloidal ferrihydrite used in these experiments was synthesized during the same co-precipitation procedure, but aliquots of the colloidal product were dialyzed in a staggered fashion over several days so that a fresh batch would be ready for each days' experiments. The ferrihydrite was added to various water samples at a concentration of approximately $2 \mu \mathrm{M} \mathrm{Fe}$. After a period of equilibration (specific details for different experiments provided below), $30 \mathrm{ml}$ aliquots of the solution were loaded into a quartz spectrophotometric cell $(10 \mathrm{~cm}$ in length) for irradiation. Two identical cells were used in experiments, each cleaned with concentrated $\mathrm{HCl}$ and ethanol and thoroughly rinsed between samples. Samples were irradiated in quartz cells in front of the light source for a maximum of 1 hour. Although all samples started at room temperature, some increase in sample temperature was observed during longer irradiation periods.

For determination of dissolved ${ }^{133} \mathrm{Ba}$ and ${ }^{59} \mathrm{Fe}$, irradiated samples and non-irradiated blanks were syringe-filtered through a $47 \mathrm{~mm}$ diameter, $0.05 \mu \mathrm{m}$ pore-size Nuclepore polycarbonate filter. Unfiltered aliquots were also taken for determination of total ${ }^{133} \mathrm{Ba}$ and ${ }^{59} \mathrm{Fe}$. All filtrate and total samples were weighed out to equal mass (about 8g) and stored in polypropylene sample cups of identical geometry for counting on our lowenergy germanium detector. An aliquot of hydroxylamine $\mathrm{HCl}$ solution $(250 \mu \mathrm{l})$ was added to all total and filtrate samples to dissolve colloidal iron and prevent wall loss.

In order to facilitate comparison with previous studies, several photochemical kinetics experiments were performed in $0.2 \mu \mathrm{m}$ filtered, aged Sargasso seawater (SSW) with $100 \mu \mathrm{M}$ citrate added. The experiments were performed at $\mathrm{pH} 8$ and at $\mathrm{pH}$ 6.5. All irradiations were performed on the same day, with the same batch of colloidal ferrihydrite. Colloids $(2 \mu \mathrm{M} \mathrm{Fe})$ were added to $\mathrm{pH} 8 \mathrm{SSW}$ with citrate, allowed to 
equilibrate for one hour, and then irradiations were performed (sequentially) for 5, 25, and 50 minutes. Blank (non-irradiated) samples were also taken for each of those time periods. The $\mathrm{pH}$ of the SSW + citrate was then adjusted to 6.5 by adding $\mathrm{HCl}$, and three more irradiations were performed (and blank samples taken) for 5, 25, and 50 minutes.

A second series of experiments was performed on a suite of natural waters collected freshly (except for SSW) from areas around Woods Hole. These waters were collected in acid-washed polycarbonate containers and stored under refrigeration for 2-3 days until used in photochemical experiments. All of the waters were used in experiments without filtration. In addition to Sargasso seawater (SSW), waters used included Vineyard Sound seawater (VSW), water from a local pond (Salt Pond), and water from a marshy area in Waquoit Bay (WB). An "estuary" sample was also made by mixing a 50/50 (volume/volume) solution of VSW and Salt Pond water. These waters (unfiltered) were all tested spectrophotometrically for their absorbance spectra between 200 and $800 \mathrm{~nm}$ (shown in Figure 4.7 for SSW, VSW, WB and Salt Pond) relative to a Milli-Q water blank. It was found that absorbance decreased in the following order:

$$
\text { Salt Pond }>>\text { "estuary" }>\text { WB }>\text { VSW }>\text { SSW }
$$

This was taken as a relative indication of the concentration of organic chromophores and suspended particulates in these waters. Information on the $\mathrm{pH}$ and salinity of each water type is provided in Table 4.1. SSW, VSW, and WB were fairly characteristic of marine waters, while the Salt Pond sample had a much lower salinity (1 ppt) and lower pH (6).

Replicate photochemical experiments were carried out in each of these water types. The ${ }^{133} \mathrm{Ba}$-ferrihydrite was added to the different water types at $2 \mu \mathrm{M} \mathrm{Fe}$ concentration, and allowed to equilibrate for a period of about one hour prior to irradiation. Samples were irradiated with the broad spectrum light source for 1 hour each, and then filtrate and total samples were taken as described above for irradiated samples and non-irradiated blanks. Due to their high organic content and the possibility of light attenuation, the Salt Pond and "estuary" samples were mixed and turned around every 15 minutes during the 1 hour irradiation. 
A

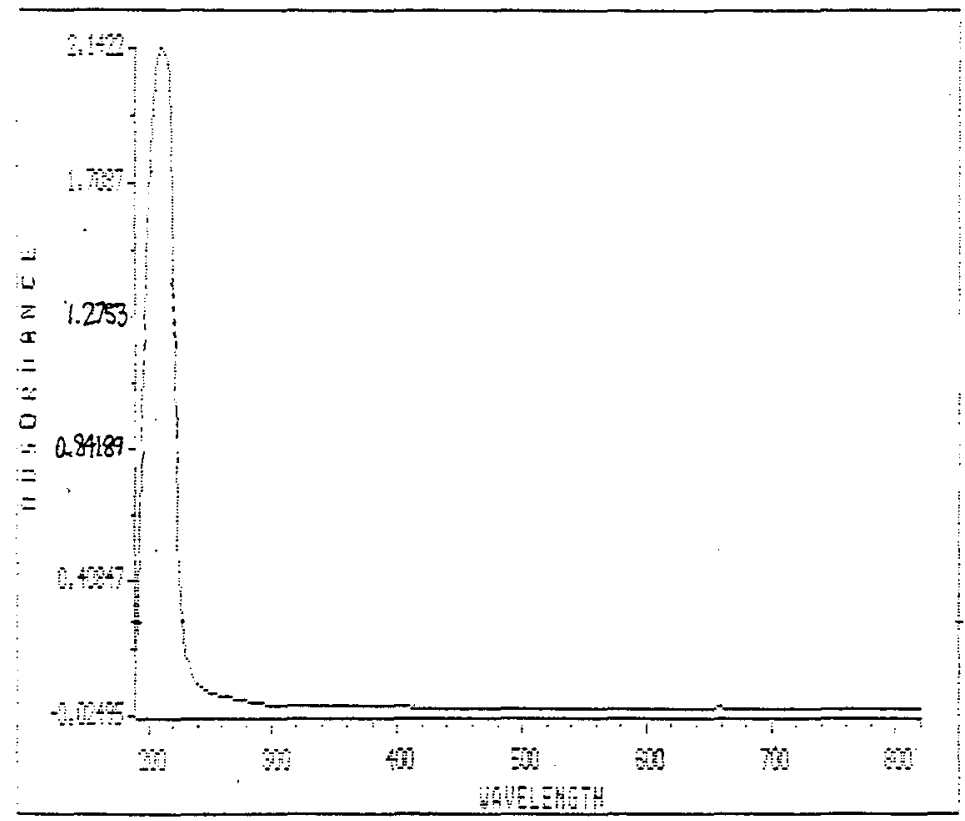

B

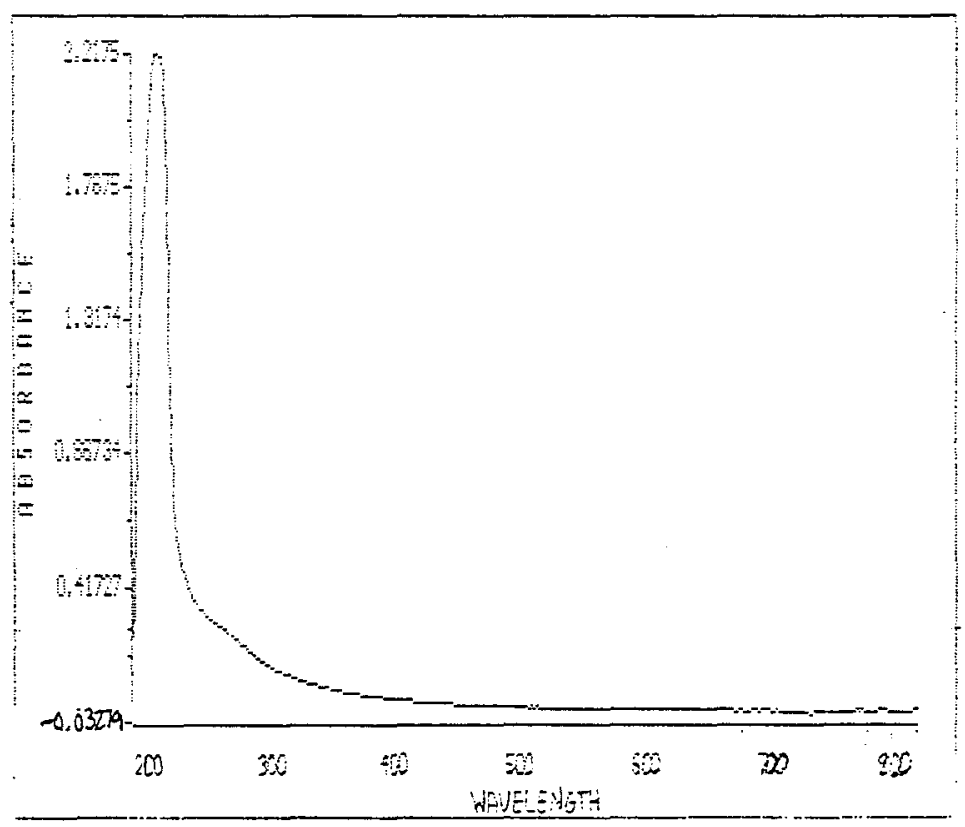

Figure 4.7 - Absorbance spectra for waters used in photochemical irradiation experiments, obtained on a Hewlett Packard 8452A Diode Array Spectrophotometer. Spectra show absorbance on the $y$-axis (relative to a Milli-Q water blank) vs. wavelength on the $x$-axis (190 to $820 \mathrm{~nm}$ ). A - Sargasso seawater (SSW); B - Vineyard Sound seawater (VSW) 


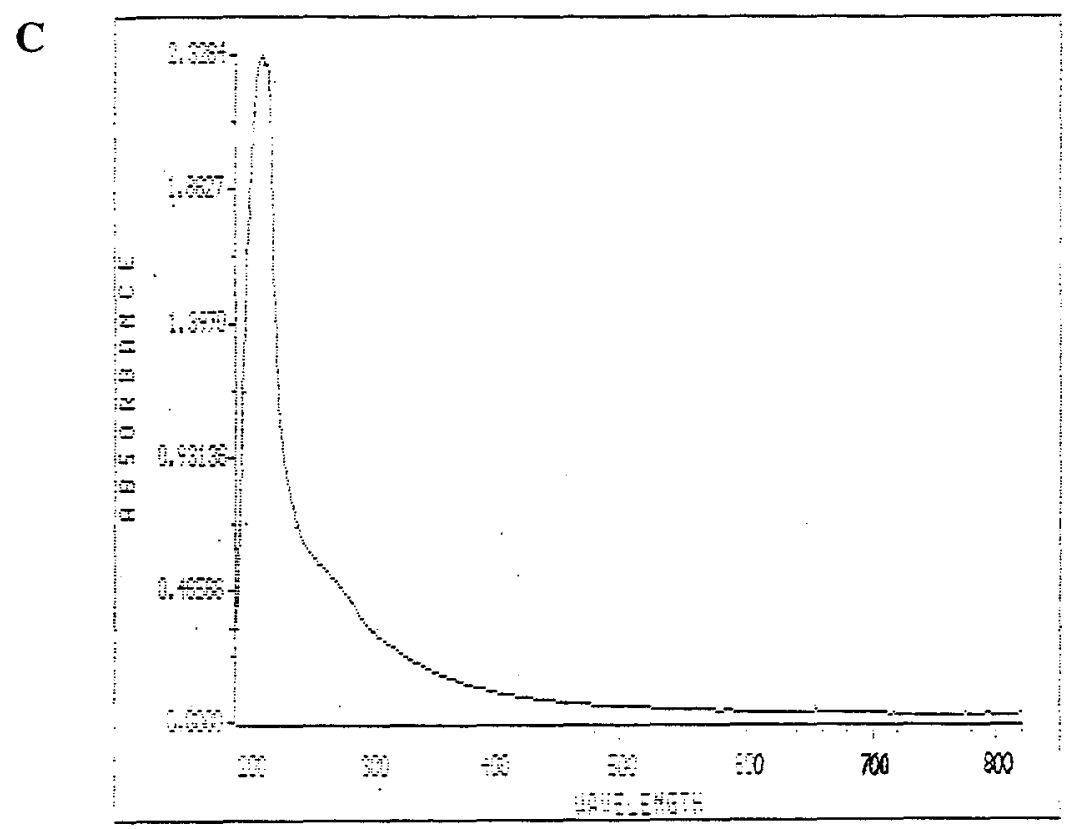

D

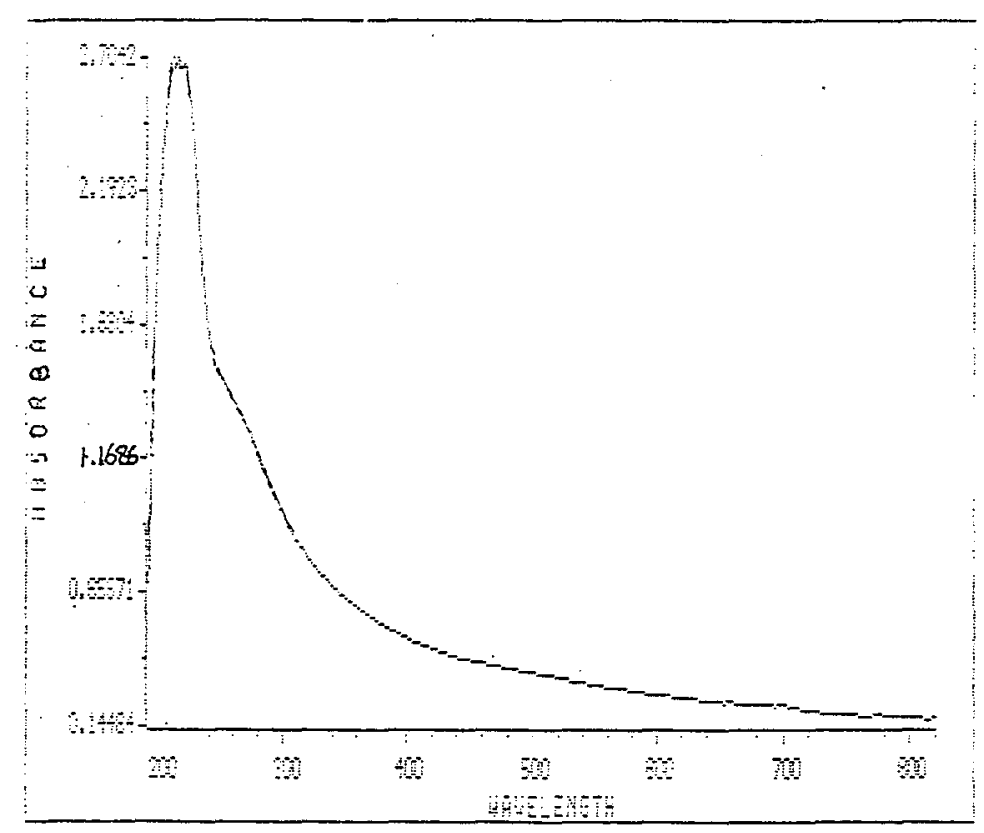

Figure 4.7, continued - C - Waquoit Bay water (WB); D - Salt Pond water 
Table 4.1 - Photochemistry in natural waters. $\%$ dissolved ${ }^{133}$ Ba produced in 1-hour $\mathrm{Hg} / \mathrm{Xe}$ lamp irradiations of ${ }^{133} \mathrm{Ba}$-ferrihydrite in various water types. Values shown for $\%{ }^{133} \mathrm{Ba}$ are the average and standard deviation of replicates. Samples above the dotted line were run on the same day, with the same batch of colloidal ferrihydrite. Samples below the line were run 2 days later, with a different batch.

\begin{tabular}{|c|c|c|c|c|}
\hline Sample & pH & $\underline{\text { Salinity (ppt) }}$ & $\begin{array}{c}\text { Blank } \\
\%{ }^{133} \mathrm{Ba}<0.05 \mu \mathrm{m} \\
\end{array}$ & $\begin{array}{c}\text { Irradiated } \\
\%{ }^{133} \mathrm{Ba}<0.05 \mu \mathrm{m}\end{array}$ \\
\hline${ }^{1} \mathrm{VSW}$ & 8 & 31 & $2.95 \pm 0.35$ & $4.85 \pm 0.35$ \\
\hline${ }^{2} \mathrm{SSW}$ & 8 & 35 & $3.10 \pm 0.14$ & $5.29 \pm 0.98$ \\
\hline${ }^{3} \mathrm{WB}$ & 8 & 24.2 & $2.85 \pm 0.07$ & $5.80 \pm 0.14$ \\
\hline "Estuary" & 7.6 & 15.4 & $2.20 \pm 0.85$ & $4.50 \pm 0.85$ \\
\hline Salt Pond & 6.0 & 1.1 & $2.95 \pm 0.35$ & $7.60 \pm 2.80$ \\
\hline
\end{tabular}

1- Vineyard Sound seawater

2- Sargasso seawater

3- Waquoit Bay water 
A second series of photochemical experiments was performed with the VSW, SSW, and WB water types. For these experiments, ${ }^{133} \mathrm{Ba}$-ferrihydrite was added to each water type $(2 \mu \mathrm{M} \mathrm{Fe})$ and allowed to incubate overnight prior to photochemical irradiation. Half of the water samples were incubated overnight at room temperature, and half were incubated in the refrigerator $\left(\sim 4^{\circ} \mathrm{C}\right)$. Irradiations were performed the next day, for one hour each, and filtrate and total samples were taken for irradiated samples and nonirradiated blanks as described above.

Results and Discussion - Figure 4.8 shows the result of the photochemical kinetics experiments in SSW $+100 \mu \mathrm{M}$ citrate at $\mathrm{pH} 8$ and $\mathrm{pH}$ 6.5. There are no replicate samples for the points on the graphs; the errors in some of the ${ }^{59} \mathrm{Fe}$ graphs are counting statistics for filtrates with very low ${ }^{59} \mathrm{Fe}$ activity. For $\mathrm{A}$ and $\mathrm{B}$, the experiments at $\mathrm{pH} 6.5$, the initial behavior of ${ }^{133} \mathrm{Ba}$ and ${ }^{59} \mathrm{Fe}$ was similar. The initial rate constant of dissolved ${ }^{133} \mathrm{Ba}$ photoproduction was $3.48 \mathrm{~h}^{-1}$; the initial rate for ${ }^{59} \mathrm{Fe}$ was similar at $3.22 \mathrm{~h}^{-1} .{ }^{59} \mathrm{Fe}$ in this system was likely stabilized in the dissolved phase by citrate complexation. The high rate constants for the period from 0 to 5 minutes decreased somewhat between 5 and 25 minutes, and the rate of dissolved ${ }^{133} \mathrm{Ba}$ production reached a plateau by 50 minutes. A decreasing photoreduction rate constant with time is consistent with observations by Wells and Mayer, 1991a, in similar experiments, and also with the work of Waite and Morel, 1984a. Such decreasing rates of photolysis with time have been attributed to depletion of organic chromophores and/or the inactivation of the Fe oxide surface by photolysis products. In our work, an additional factor may be the depletion of ${ }^{133} \mathrm{Ba}$ at the iron oxide surface. The large drop in dissolved ${ }^{59} \mathrm{Fe}$ at 50 minutes in the $\mathrm{pH} 6.5$ experiment is difficult to explain. It could be due to loss of citrate by photolysis due to the extended irradiation period and the intense iron redox cycling in this system, despite the initial excess of citrate relative to Fe. Further work is needed to confirm the validity of this interpretation.

In graphs $\mathrm{C}$ and $\mathrm{D}$, for the experiment at $\mathrm{pH} 8$, an increase in dissolved ${ }^{133} \mathrm{Ba}$ was also observed due to photoreduction, and the initial rate constant $\left(0.14 \mathrm{~h}^{-1}\right)$ was about $25 \mathrm{x}$ less 

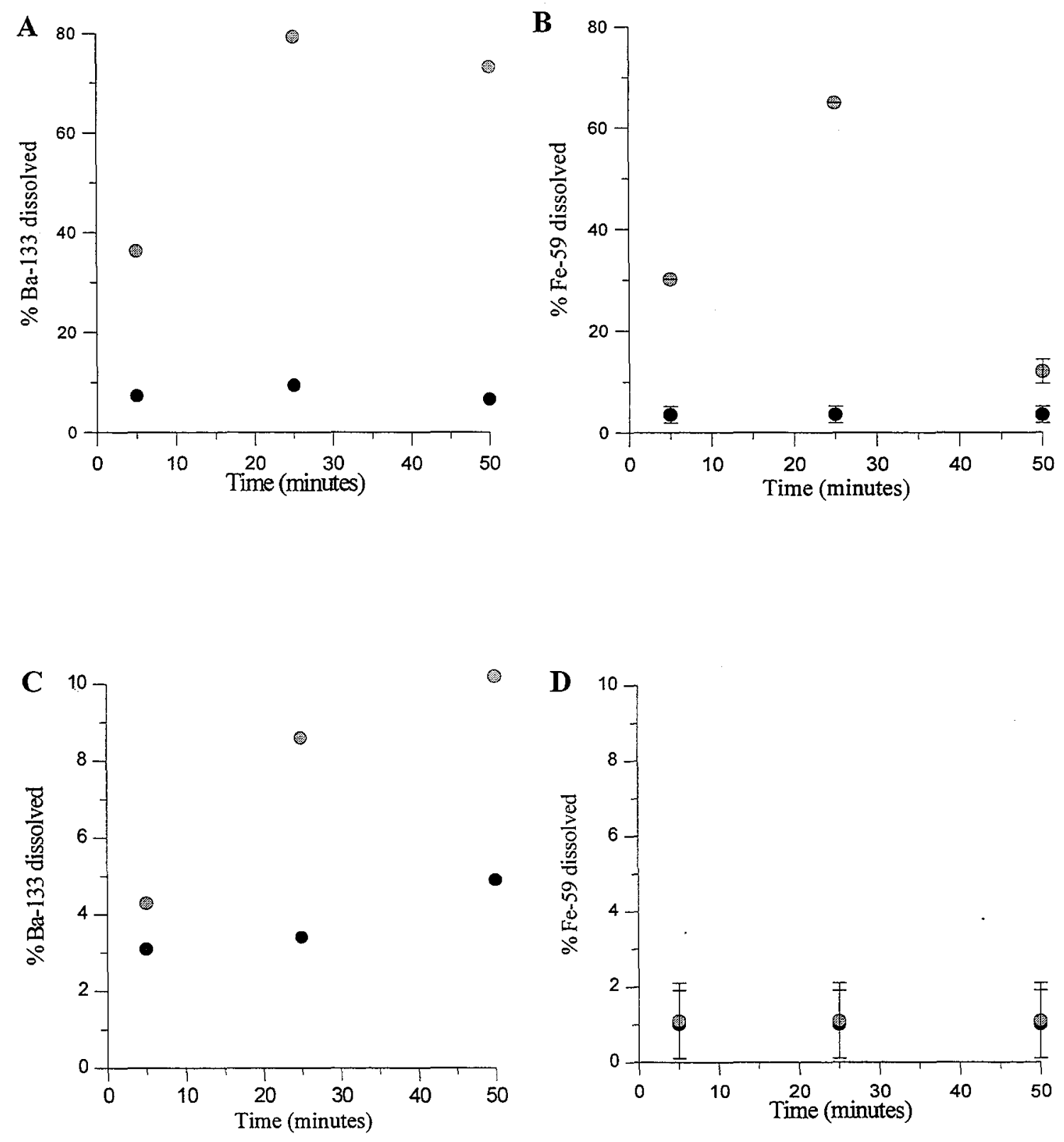

Figure 4.8 - Photochemical kinetics experiment in SSW + 100uM citrate, $\mathrm{pH} 6.5$ and 8. A - \% dissolved $\mathrm{Ba}-133$ vs time at $\mathrm{pH} 6.5$ $\mathrm{B}-\%$ dissolved $\mathrm{Fe}-59$ vs time at $\mathrm{pH} 6.5$ $\mathrm{C}-\%$ dissolved $\mathrm{Ba}-133$ vs time at $\mathrm{pH} 8$ $\mathrm{D}-\%$ dissolved $\mathrm{Fe}-59$ vs time at $\mathrm{pH} 8$

- Blank

- Irradiated 
than that observed at $\mathrm{pH}$ 6.5. This is consistent with the results of Waite and Morel, 1984a, who found about $8 x$ less filterable iron produced during irradiation of lepidocrocite at $\mathrm{pH} 8.2$ vs. $\mathrm{pH} 6.5$, thus indicating a lower dissolution rate. The lowered dissolution rate of iron oxide at $\mathrm{pH} 8$ relative to $\mathrm{pH} 6.5$ is a consequence of both the reduced affinity of citrate for the iron oxide surface at higher $\mathrm{pH}$ (Waite and Morel, 1984a), and also the strong $\mathrm{pH}$ dependence of the oxidation rate of Fe(II) (Moffett and Zika, 1987). Dissolved ${ }^{59} \mathrm{Fe}$ in the irradiated sample demonstrated no increase relative to the non-irradiated blank, within counting error. At this $\mathrm{pH}$, photoreduced iron is most likely being recycled rapidly at the surface of the Fe oxide, without going into solution. This interpretation has been advanced before by previous workers (Wells and Mayer, 1991a). Although iron did not accumulate measurably in solution, photochemical reactions at the oxide surface did apparently result in a release of ${ }^{133} \mathrm{Ba}$ to solution. A decrease in the photoproduction rate of dissolved ${ }^{133} \mathrm{Ba}$ was observed at this $\mathrm{pH}$, but unlike the experiment at $\mathrm{pH} 6.5$ the initial rate (from 0-5 min.) did not decrease until after 25 minutes of irradiation and the photoproduction rate did not plateau between 25 and 50 minutes. The more prolonged photoproduction of dissolved ${ }^{133} \mathrm{Ba}$ at $\mathrm{pH} 8$ relative to pH 6.5 may be due to the lower rate of iron redox cycling in this system, concurrent with lower rates of citrate photolysis and surface inactivation than at $\mathrm{pH} 6.5$.

The results of the 1 hour irradiations after brief equilibration in VSW, SSW, WB, "estuary" and Salt Pond are shown in Table 4.1. All of these waters exhibited some degree of photoreactivity, as indicated by higher dissolved ${ }^{133} \mathrm{Ba}$ activity in the irradiated samples. (No increase in dissolved ${ }^{59} \mathrm{Fe}$ was detected.) For most of these waters, the averaged rate constant of dissolved ${ }^{133} \mathrm{Ba}$ photoproduction over the 1 hour irradiation period was between 0.02 and $0.03 \mathrm{~h}^{-1}$, assuming linear kinetics. The exception was the Salt Pond sample, which had an average rate constant of $0.05 \mathrm{~h}^{-1}$, possibly due to the higher concentration of dissolved chromophores (Fig. 4.7) as well as the lower $\mathrm{pH}$ in this water type relative to the others. The similarity of the results for VSW, SSW, and WB suggest that, for these waters, there was not a strong relationship between photoreduction rate and the concentration of natural organic chromophores present. This is consistent 
with the results of Wells and Mayer, (1991a), who found no difference in photoreduction rates of colloidal iron oxides between Maine coastal seawater and Sargasso seawater.

The 1 hour averaged rate constant for SSW $\left(0.02 \mathrm{~h}^{-1}\right)$ in this data set is substantially less than the 50 minute averaged rate constant for SSW + citrate at pH 8 in Figure 4.8 $\left(0.085 \mathrm{~h}^{-1}\right)$. This suggests that the presence of effective chromophores like citrate can make a difference in photoreduction rate. This is not exactly an equal comparison, since these rate constants are not actually first order, and the averaged time period is slightly different in each case. Nonetheless, the comparison suggests that the addition of citrate significantly increased the rate of photoreduction at $\mathrm{pH} 8$ in SSW. This is in agreement with the work of Waite and Morel, 1984a, who found a proportional relationship at pH 6 between the photoconversion rate and concentration of citrate in the system. Wells and Mayer, 1991a, did not see any difference in photoproduction rate as measured by oxine reactivity in systems with and without added citrate. This difference may be due to the different methods used by Wells and Mayer and Waite and Morel to measure the rate of photoreduction.

The final data set for this series is shown in Figure 4.9, for ferrihydrite equilibrated overnight in VSW, SSW, and WB at room temperature and at $4^{\circ} \mathrm{C}$. In terms of the photoproduction of dissolved ${ }^{133} \mathrm{Ba}$ over a one hour irradiation, the increased equilibration time relative to the data in Table 4.1 did not make a big difference. Averaged rates still varied between $0.02 \mathrm{~h}^{-1}$ and $0.03 \mathrm{~h}^{-1}$ for all these samples, with the ones incubated at $4^{\circ} \mathrm{C}$ being slightly higher than the ones incubated at room temperature. This data indicates that the timescale for adsorption of organic chromophores onto the iron oxide surfaces is not limiting to the photoreduction observed after equilibrations of an hour or more. Adsorption of organics by the colloidal ferrihydrite must therefore be relatively rapid, as hypothesized by Wells and Mayer, (1991a).

The most striking difference in this experiment was in the non-irradiated blanks, between those equilibrated overnight at $4^{\circ} \mathrm{C}$ vs those equilibrated at room temperature (Fig. 4.9). The room temperature blanks were at least twice as high, possibly due to higher rates of thermal dissolution/maturation reactions at room temperature relative to 


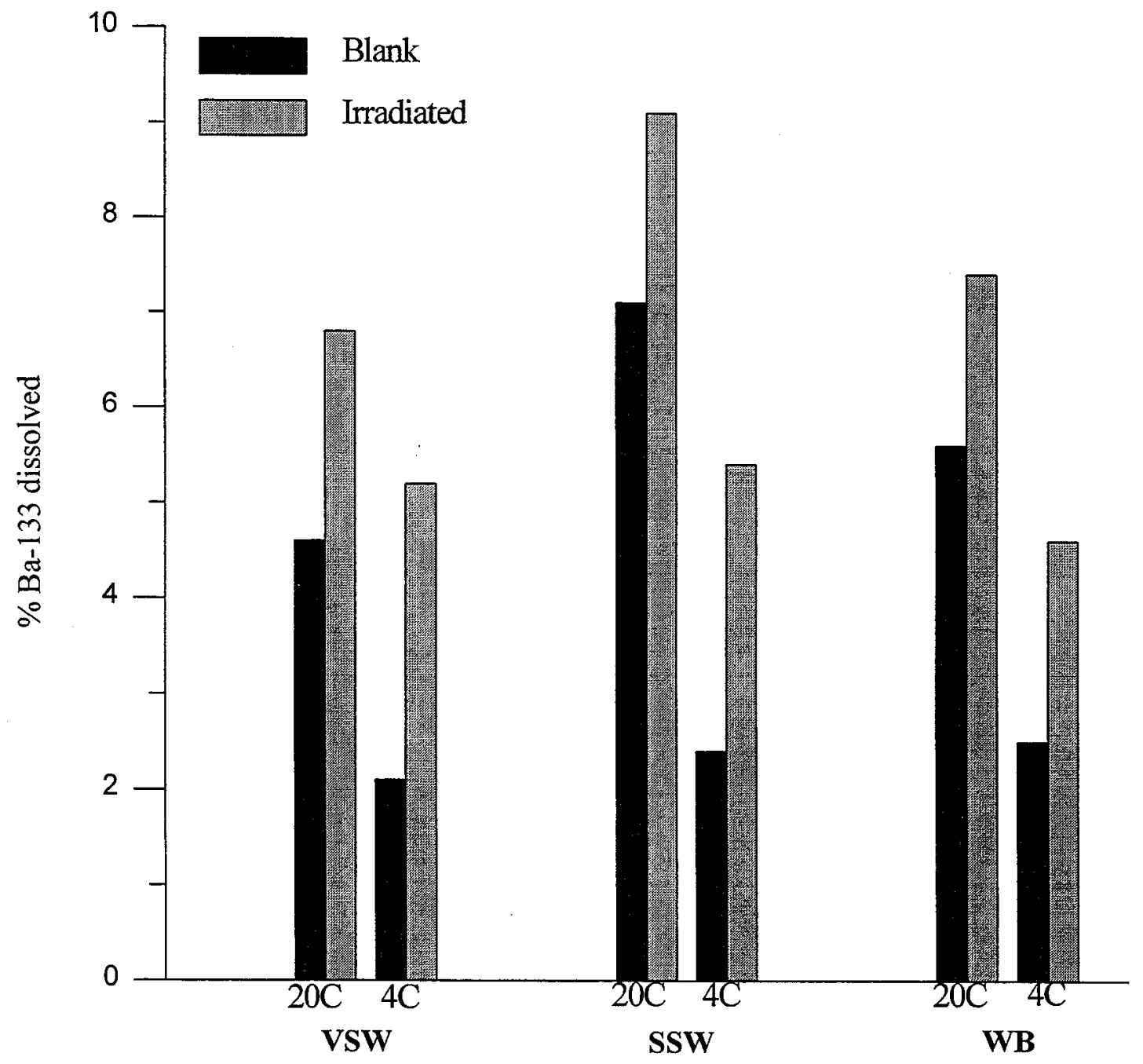

Figure 4.9 - Photochemical and thermal effects.

$\%$ dissolved $\mathrm{Ba}-133$ produced upon irradiation of ferrihydrite incubated overnight at 4 vs 20 degrees $C$ in Vineyard Sound seawater (VSW), Sargasso seawater (SSW) and Waquoit Bay water (WB).

Irradiation time 1 hour. 
$4^{\circ} \mathrm{C}$. These water samples were unfiltered, however, so the possible effects of biota on the reactivity of the colloidal iron oxides cannot be excluded. The room temperature blank for SSW appeared to be significantly higher than for VSW and WB. It is possible that the reaction rate was affected by the presence of specific organic ligands in the different water types.

A related series of experiments was conducted some months later, equilibrating ${ }^{133} \mathrm{Ba}-$ colloidal ferrihydrite for 24 hours at room temperature and at $4^{\circ} \mathrm{C}$ in replicate containers of various water types and comparing the $\%$ of filterable ${ }^{133} \mathrm{Ba}$ produced at the end of the equilibration period. These results are shown in Table 4.2. Again, the room temperature equilibrations had significantly higher blanks than the $4^{\circ} \mathrm{C}$ equilibrations. The room temperature blank in $0.2 \mu \mathrm{m}$ filtered VSW was not much lower than that in unfiltered VSW, and poisoning the unfiltered VSW with azide made little difference. This indicates that biological processes were probably not respondible for the difference observed between $20^{\circ} \mathrm{C}$ and $4^{\circ} \mathrm{C}$ samples. The room temperature blank for filtered SSW was lower than that for filtered, UV-irradiated SSW (UVSSW), indicating that the dissolution was not mediated by dissolved organics. The higher reaction rate observed at room temperature was thus most likely due to thermal dissolution/maturation of the colloidal ferrihydrite. Maturation via hydrolysis/polymerization has been observed before as a decrease over time in the level of oxine-reactive iron (Wells and Mayer, 1991a) and reducible iron (Johnson et. al., 1994) during dark incubation. The strong temperature dependence of this process has not previously been noted. The higher rate of reaction in SSW relative to VSW and WB may have been due to lower concentrations of phosphate and silicate in the oligotrophic water of the Sargasso Sea relative to coastal waters. These anions are known to interact with the iron oxide surface, lowering its reactivity (Moffett and Zafiriou, 1993; Bondietti et. al., 1993).

Photochemical experiment in natural sunlight - A photochemical experiment was conducted with ${ }^{133} \mathrm{Ba}$-ferrihydrite in natural sunlight in fresh Vineyard Sound seawater on July 8th, 1997. VSW was collected on an incoming tide the previous day, in an acid- 
Table 4.2 - Thermal effects. $\%$ dissolved ${ }^{133} \mathrm{Ba}$ in samples incubated 24 hours at $4{ }^{\circ} \mathrm{C}$ vs samples incubated 24 hours at $20^{\circ} \mathrm{C}$.Values shown are the average and standard deviation of replicates. Samples above the dotted line were run on the same day, with the same batch of colloidal ferrihydrite. Samples below the line were run on a different day, with another batch of colloids.

\begin{tabular}{|c|c|c|}
\hline Sample & $\begin{array}{c}4^{\circ} \mathrm{C} \\
\%^{133} \mathrm{Ba}<0.05 \mu \mathrm{m}\end{array}$ & $\begin{array}{c}20^{\circ} \mathrm{C} \\
\%{ }^{133} \mathrm{Ba}<0.05 \mu \mathrm{m}\end{array}$ \\
\hline$<0.2 \mu \mathrm{m} \mathrm{VSW}$ & $1.20 \pm 0.14$ & $2.92 \pm 0.03$ \\
\hline whole VSW & $1.50 \pm 0.42$ & $3.55 \pm 0.21$ \\
\hline whole VSW + azide & ---- & $2.75 \pm 0.64$ \\
\hline$<0.2 \mu \mathrm{m} \mathrm{SSW}$ & $1.50 \pm 0$ & $4.60 \pm 1.1$ \\
\hline UVSSW & $1.60 \pm 0$ & $6.10 \pm 0.4$ \\
\hline
\end{tabular}


cleaned polycarbonate bottle. Half of the VSW was filtered through an acid-cleaned, $0.2 \mu \mathrm{m}$ pore size Nuclepore polycarbonate filter. ${ }^{133} \mathrm{Ba}$-impregnated colloidal ferrihydrite (co-labeled with ${ }^{59} \mathrm{Fe}$ ) was added to both filtered and unfiltered VSW $(\sim 3 \mu \mathrm{M} \mathrm{Fe})$, and equilibrated overnight at $4^{\circ} \mathrm{C}$. At 10 am the next morning, both filtered and unfiltered VSW/ferrihydrite solutions were placed in acid-cleaned quartz flasks and irradiated under natural sunlight until $4 \mathrm{pm}$. During the irradiation, the flasks were submerged (up to the neck) in a circulating water bath which maintained the water temperature at $19^{\circ} \mathrm{C}$ throughout the day. Foil-wrapped flasks, as dark controls, were also submerged in the water bath. Irradiated flasks and dark controls were sampled at intervals for dissolved and total ${ }^{133} \mathrm{Ba}$ and ${ }^{59} \mathrm{Fe}$ as described in previous experiments. There were no replicates in this experiment.

Both filtered and unfiltered VSW flasks demonstrated evidence of photoproduction of dissolved ${ }^{133} \mathrm{Ba}$ relative to the dark controls (Fig. 4.10). No dissolved ${ }^{59} \mathrm{Fe}$ was observed in any filtrates. The initial rate constant for production of dissolved ${ }^{133} \mathrm{Ba}$ from $10 \mathrm{am}$ to 11:30am was $0.005 \mathrm{~h}^{-1}$ in unfiltered VSW, and $0.003 \mathrm{~h}^{-1}$ in filtered VSW. The initial rate dropped slightly in the unfiltered VSW, and remained relatively linear in the filtered VSW over the course of the irradiation. These initial rate constants are about an order of magnitude lower than what was observed for photochemistry in VSW using the irradiation system. This is consistent with the $\sim 10 \mathrm{x}$ lower light intensity in natural sunlight relative to the $1000 \mathrm{~W} \mathrm{Hg} / \mathrm{Xe}$ lamp. Waite and Morel, 1984a, observed a linear relationship between light intensity and photoreduction rate. Wells and Mayer, 1991a did not see differences in photoreduction rate at different light intensities.

The rate of photoproduction of dissolved ${ }^{133} \mathrm{Ba}$ was higher in the unfiltered VSW than in the filtered VSW. This is consistent with the work of Johnson et. al., 1994, who observed larger diurnal cycling in total reducible iron as a result of photochemical processes in unfiltered vs. filtered equatorial Pacific water. The higher rate of reaction may be due to the interaction of colloidal ferrihydrite with particle surfaces. It is possible that the presence of additional adsorptive surfaces for photoproduced dissolved $\mathrm{Fe}$ species allows a greater chance for ${ }^{133} \mathrm{Ba}$ to escape from the Fe oxide lattice. Particles 


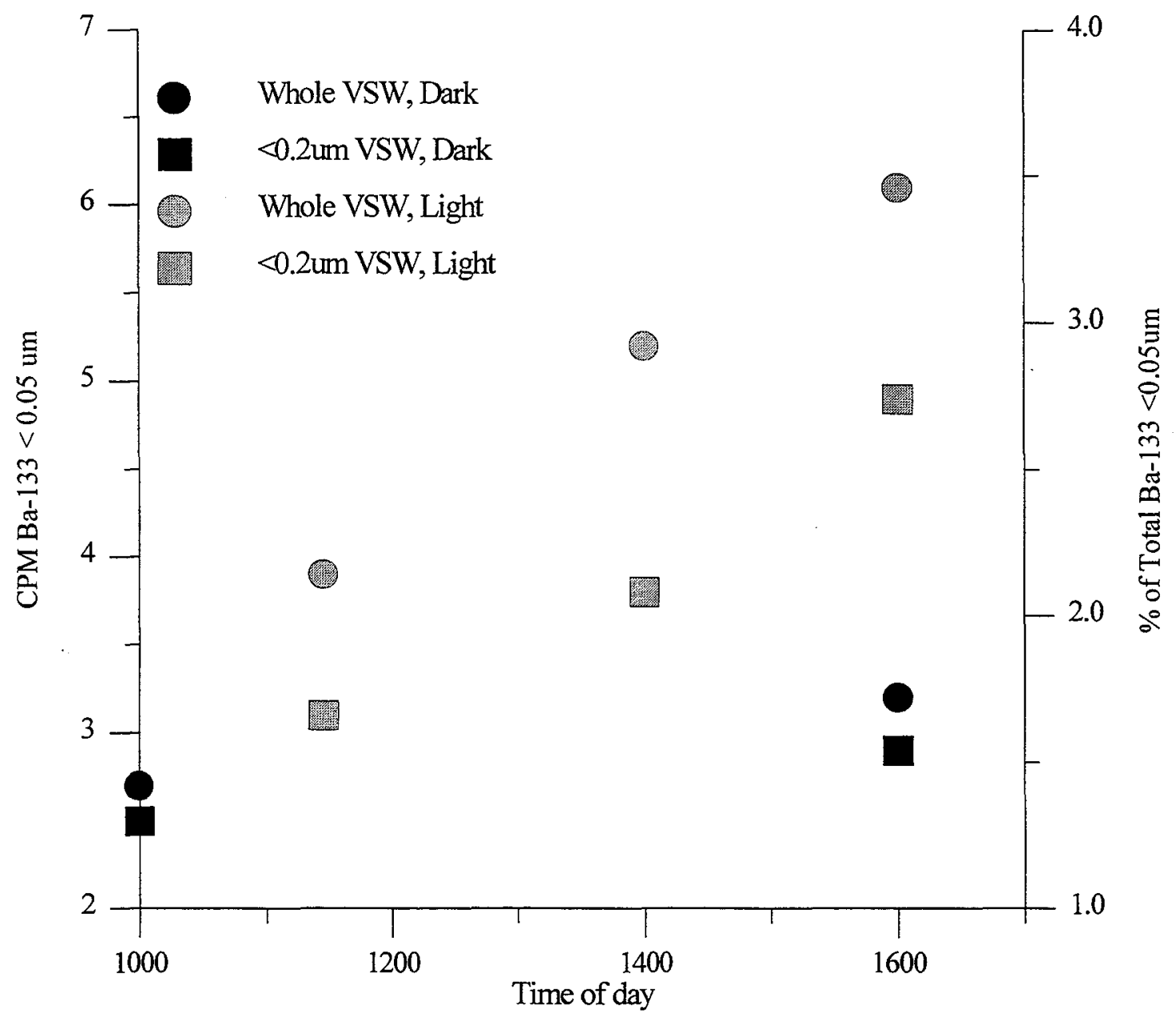

Figure 4.10 - Photochemical experiment, 7/8/97.

Natural sunlight irradiation of $\mathrm{Ba}-133$ ferrihydrite in filtered and unfiltered Vineyard Sound seawater. left $y$-axis - CPM Ba- $133<0.05 u m$ right $\mathrm{y}$-axis $-\%$ of total $\mathrm{Ba}-133<0.05$ um 
might also increase reaction rates by providing a source of redox-active organic material close to iron oxide surfaces.

The initial rate of photoproduction of dissolved ${ }^{133} \mathrm{Ba}$ in unfiltered VSW $\left(0.005 \mathrm{~h}^{-1}\right)$ is a factor of 3-4 less than the oxine lability photoreduction rate measured by Wells and Mayer, (1991a) for 5 $\mu$ M 90 FERR incubated in Damariscotta surface seawater under natural noontime sun in July 1988 , in a flowing seawater bath at $13.5^{\circ} \mathrm{C}$ (Wells and Mayer 1991a; rate constant estimated from Fig. $5=0.016-0.018 \mathrm{~h}^{-1}$ ). Considering the differences between the ${ }^{133} \mathrm{Ba}$ method and the oxine lability method, the two rates agree fairly well. There are a number of reasons, aside from intrinsic differences between the two techniques, why our observed rate constant could be lower. Our colloids are almost certainly more refractory than those used by Wells and Mayer. Our $90^{\circ} \mathrm{C}$ ferrihydrite colloids were dialysed at $\mathrm{pH} 5$ for 60 hours and equilibrated overnight in VSW prior to irradiation, whereas Wells and Mayer synthesized their $90^{\circ} \mathrm{C}$ ferrihydrite and added it directly to the water sample just prior to the experiment. As a "fresher" phase, the Wells and Mayer ferrihydrite is very likely to contain more reactive iron than our colloids, and thus should show a higher degree of photoreactivity as demonstrated by a faster initial photoreduction rate.

Additional factors may partially account for the difference in measured rate of photoreduction. The Wells and Mayer data is for noon-time sun, whereas our initial rate constant was derived between 10 and 11:30am, when the sunlight is somewhat less intense. Finally, the temperature of incubation was about $5.5^{\circ} \mathrm{C}$ lower in the Wells and Mayer experiment. The rate of Fe(II) oxidation is temperature-dependent (Moffett and Zika, 1987), and a longer lifetime of Fe(II) could increase the apparent rate of iron oxide dissolution.

It is interesting to compare this experiment to our earlier photochemistry results with ${ }^{51} \mathrm{Cr}$ (VI)-impregnated ferrihydrite irradiated under natural sunlight (Figure 4.2). The initial photoproduction rate of dissolved ${ }^{51} \mathrm{Cr}$ in those experiments was $0.04-0.05 \mathrm{~h}^{-1}$, which is much higher than the rate observed for dissolved ${ }^{133} \mathrm{Ba}$ photoproduction in natural sunlight (Fig. $4.10 ; 0.005 \mathrm{~h}^{-1}$ in unfiltered VSW). The reason for this difference is 
unclear, and may be due to factors such as colloid reactivity and concentrations of organic chromophores present in the different experiments. Alternatively, the difference may be related to the nature of $\mathrm{Cr}(\mathrm{VI})$ incorporation into the iron oxide phase. As an oxyanion, $\mathrm{CrO}_{4}{ }^{2-}$ is much larger than $\mathrm{Ba}^{2+}$. It is possible that the removal of $\mathrm{CrO}_{4}{ }^{2-}$ from the iron oxide matrix leaves a large "hole" which exposes more reactive iron to photochemical reaction. The known redox chemistry between $\mathrm{Cr}(\mathrm{VI})$ and $\mathrm{Fe}$ at the iron oxide surface could also affect the apparent rate of oxide dissolution as measured by production of dissolved ${ }^{51} \mathrm{Cr}$. Further work is necessary to substantiate these hypotheses.

\section{${ }^{133} \mathrm{Ba}$-impregnated ferrihydrite - colloids rinsed via filtration/resuspension}

The feasibility of rinsing excess ${ }^{133} \mathrm{Ba}$ out of the ${ }^{133} \mathrm{Ba}$-impregnated colloidal ferrihydrite by a filtration/resuspension protocol instead of by dialysis was investigated. The possible advantages of this procedure include less time- consuming ferrihydrite preparation, generation of less radioactive waste during rinsing, and potentially a more reactive ferrihydrite phase. Filtration/resuspension procedures were carried out by filtering the concentrated colloidal ferrihydrite onto a $0.2 \mu \mathrm{m}$ pore size Nuclepore polycarbonate filter, rinsing several times, and resuspending the colloidal ferrihydrite in a small volume of solution. This concentrate was then ultra-sonicated, allowed to equilibrate for some period of time, and the filtration/rinsing/resuspension procedure was repeated.

Generally, 4-5 repetitions of this procedure, with one extended equilibration period (several hours) between the 4th and 5th rinse resulted in colloids that had a low enough ${ }^{133} \mathrm{Ba}$ blank to be used in photochemical studies. Preliminary experiments with these colloids using the $\mathrm{Hg} / \mathrm{Xe}$ lamp irradiation system described previously indicated that they were more reactive than dialysis-rinsed ${ }^{133} \mathrm{Ba}$-colloids. For 1 hour irradiations in VSW and 30 minute irradiations in SSW+citrate at $\mathrm{pH} 8$, the filtration/resuspension-rinsed ferrihydrite demonstrated averaged dissolved ${ }^{133} \mathrm{Ba}$ photoproduction rates of $0.06 \mathrm{~h}^{-1}$ and $0.21 \mathrm{~h}^{-1}$, respectively. (Compared to respective rate constants of $0.02 \mathrm{~h}^{-1}$ and $0.08 \mathrm{~h}^{-1}$ for dialysis-rinsed ferrihydrite under similar conditions). 
Achieving a consistently low ${ }^{133} \mathrm{Ba}$ blank was found to be a problem with the filtration/resuspension rinsing protocol. Particularly over extended equilibration in seawater samples (12 hours or longer), the ${ }^{133} \mathrm{Ba}$ blank of ferrihydrite rinsed via filtration/resuspension increased to levels of $15-20 \%$. With blanks this high, it was not possible to see any changes in dissolved ${ }^{133} \mathrm{Ba}$ upon photoirradiation. This may have been due to depletion of ${ }^{133} \mathrm{Ba}$ at the surface of the colloidal ferrihydrite.

In order to reduce the blank over extended periods in seawater, the filtration/resuspension protocol was altered by rinsing in various media, and extending the equilibration period between rinses. Seawater and Milli-Q water were about equally effective used as rinsing media. By equilibrating overnight in seawater before the final rinse, ferrihydrite which maintained a lower ${ }^{133} \mathrm{Ba}$-blank over longer periods of time could be achieved. These colloids still had much higher blank values than the dialyzed ${ }^{133} \mathrm{Ba}$-impregnated ferrihydrite, but their greater level of reactivity made them better suited to some applications.

Figure 4.11 shows preliminary results from an experiment in which ${ }^{133} \mathrm{Ba}-$ impregnated, filtration/resuspension rinsed colloids were added to replicate bacterial suspensions $\left(\sim 5 \times 10^{7}\right.$ cells $/ \mathrm{ml}$, Halomonas halodurans $)$ both with and without the addition of azide (metabolic poison). At intervals, these cultures were sampled for dissolved $(<0.05 \mu \mathrm{m})$ and total ${ }^{133} \mathrm{Ba}$. Over a 48 hour period, the slope of the increase in dissolved ${ }^{133} \mathrm{Ba}$ in the bacterial suspensions without azide was almost twice the slope in the poisoned cultures. Although the difference between azide-treated and non-azidetreated supensions was fairly subtle, this is tentative evidence for bacterially-mediated dissolution of colloidal ferrihydrite. Repeated attempts failed to reproduce these results with ${ }^{133} \mathrm{Ba}$-impregnated ferrihydrite rinsed by dialysis. If $H$. halodurans is able to affect the reactivity of colloidal ferrihydrite, its influence can only be detected on exceedingly labile phases, such as those produced by the filtration/resuspension rinse protocol.

While these results are suggestive, they are very preliminary. It is difficult to "poise" the reactivity of the ${ }^{133} \mathrm{Ba}$-impregnated colloids at a level such that very slight dissolution effects can be detected over the elevated ${ }^{133}$ Ba-blanks common with highly reactive iron 


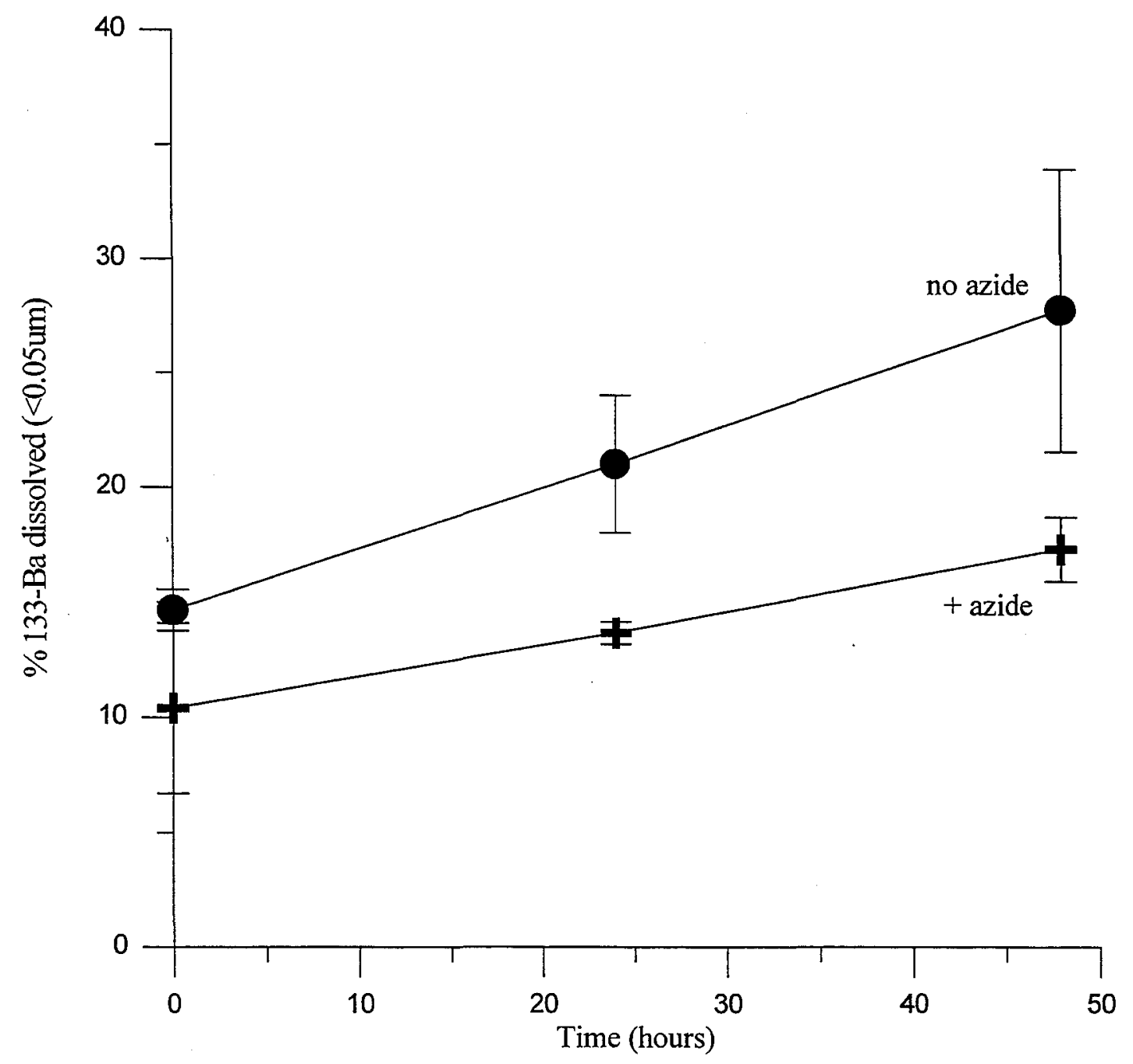

Figure 4.11 - Bacterial dissolution experiment

Filtration/resuspension-rinsed 133-Ba ferrihydrite added to bacterial suspensions with and without azide $\%$ dissolved $\mathrm{Ba}-133$ vs time 
oxide phases. This is particularly difficult in systems involving biological surface area, and extended incubation times. It may be possible to develop the filtration/resuspension rinsed ${ }^{133} \mathrm{Ba}$-ferrihydrite for such applications, but that work is beyond the scope of this thesis. More developmental studies need to be done on the filtration/resuspension protocol, and its effects on colloid reactivity. In addition, co-dissolution experiments need to be performed with the filtration/resuspension-rinsed ferrihydrite in order to determine how uniformly the ${ }^{133} \mathrm{Ba}$ is incorporated into the softer iron oxide surface. For our further work on protozoan grazer-mediated dissolution of colloidal iron oxides (Chapter 5), the filtration/resuspension rinsing protocol was abandoned in favor of the dialysis rinsing protocol, which produces ${ }^{133} \mathrm{Ba}$-impregnated colloidal ferrihydrite of a lower but more predictable level of reactivity that is better suited to extended incubations with grazers and bacteria.

\section{Conclusion}

In this chapter, a novel technique for studying reactions of synthetic iron oxide phases in seawater was presented. This technique is based on the synthesis of colloidal ferrihydrite uniformly impregnated with the radiotracer ${ }^{133} \mathrm{Ba}$. A variety of co-dissolution experiments have shown that the tracer is evenly distributed throughout the iron oxide matrix. In seawater, when the oxide structure is altered by chemical reactions, ${ }^{133} \mathrm{Ba}$ is released into the dissolved phase due to a combination of low affinity for the oxide surface and ion exchange with $\mathrm{Mg}^{2+}$ and $\mathrm{Ca}^{2+} \cdot{ }^{133} \mathrm{Ba}$ remains in the dissolved phase because it is generally non-particle reactive. The rate of release of ${ }^{133} \mathrm{Ba}$ in a given system provides a relative measure of the rate at which the iron oxide phase is reacting.

This technique has been applied in studies of the photoreduction of colloidal ferrihydrite in seawater under different conditions of $\mathrm{pH}$, organic chromophore concentration, and light intensity. Our results are generally consistent with previous studies of the photoreduction of iron oxides in seawater by Waite and Morel, 1984a and b, and Wells and Mayer, 1991a. The rate of photoreduction in our experiments, as 
measured by the production of dissolved ${ }^{133} \mathrm{Ba}$, was positively correlated with organic chromophore concentration and light intensity, and negatively correlated with $\mathrm{pH}$. The technique is sufficiently sensitive to measure the photoreduction of iron oxides at $\mu \mathrm{M}$ concentration in coastal seawater under natural sunlight.

Additional experiments were performed to study the reaction of iron oxides in the dark, at different temperatures. ${ }^{133} \mathrm{Ba}$ was found to be a sensitive indicator of the extent of iron oxide reaction under such conditions. Dark reactions of colloidal ferrihydrite demonstrate a strong temperature dependence within a range of $4-20^{\circ} \mathrm{C}$, and these reactions appear to occur independently of the presence of organic material. Release of ${ }^{133} \mathrm{Ba}$ may occur due to either thermal maturation processes, thermal dissolution, or a combination of both. In addition to temperature, the extent of dark reaction may also be influenced by the concentration of inorganic ligands, particularly phosphate, present in seawater.

The inert tracer method, using ${ }^{133}$ Ba-impregnated colloidal ferrihydrite, provides information comparable to other methods that have been used to study the reaction of iron oxides in seawater. In addition, this technique offers some advantages compared to other methods. As mentioned in the introduction, one benefit of the method is its utility for studying relatively slow reactions of iron oxides, or reactions that take place in heterogeneous systems, such as at the particle/water or cell/water interface. The ${ }^{133} \mathrm{Ba}-$ technique involves no addition of strong iron chelators, no reduction in $\mathrm{pH}$, and no addition of reducing agents. It therefore provides direct information about the extent of reaction of iron oxides at truly ambient conditions. No extrapolation from the conditions of the analytical method to ambient conditions is required. The ${ }^{133} \mathrm{Ba}-$ method works equally effectively for a variety of iron oxide reaction mechanisms, including complexation, reduction, and proton-mediated dissolution. This may be a significant advantage, given the current emphasis in the field on the role of strong-binding ligands such as siderophores in the marine chemistry of iron (eg. Rue and Bruland, 1995; Haygood et. al., 1993). The effect of such ligands on the dissolution of iron oxide phases is not detectable by competitive ligand exchange methods or $\mathrm{Fe}(\mathrm{II})$ techniques. The ${ }^{133} \mathrm{Ba}$ 
method could provide a direct measurement of the influence of siderophore-like compounds on iron oxide solubility.

The inert tracer technique also has some disadvantages relative to other methods, the primary one being that it necessitates the use of synthetic iron oxide phases. The method also provides no direct information about iron speciation in the system, although phases co-labeled with ${ }^{133} \mathrm{Ba}$ and ${ }^{59} \mathrm{Fe}$ do provide information about iron as ${ }^{59} \mathrm{Fe}$. A final disadvantage is that the mechanistics of ${ }^{133} \mathrm{Ba}$ incorporation into the iron oxide phase, and its release upon reaction in seawater, are thus far not well-constrained. Some of the uncertainty regarding these processes could be eliminated by further study.

Although used in this work at $\mu \mathrm{M} \mathrm{Fe}$ concentrations, the ${ }^{133} \mathrm{Ba}$ method can be used at lower Fe concentrations. The ultimate limitation on sensitivity is the detection limit of the impregnated tracer. It is possible that a different tracer, with a lower detection limit, could be incorporated into the iron oxide and thus lower the detection limit for the technique even further. At a certain level, particularly with ferrihydrite which forms very small subunits, size fractionation of the colloidal iron oxide from the dissolved tracer in seawater becomes an issue. We have successfully applied dialysis techniques in combination with the inert tracer method (see Chapter 5), which allows a much lower size cut-off than conventional filtration with a membrane filter as used in the experiments in this chapter.

The inert tracer technique for studying reactions of iron oxide as described in this chapter demonstrates considerable promise, particularly for mechanistic studies of iron oxide reactions in complex systems where the fate of iron itself is not well-constrained. This includes studies of the biological mobilization of refractory iron solid phases in natural waters. Thus far very little is known about such processes, although indications are that such phenomena could play a key role in marine ecosystems. There is increasing recognition, for example, of the potential importance of iron, particularly iron solids, in determining primary productivity in coastal systems. The solid phase can represent an important reservoir of bioavailable iron in coastal environments, and biota are likely to take an active part in its acquisition (Johnson et. al., 1997; Wells and Mayer, 1991b). In 
another example from the open oceans, recent realization of the importance of nitrogenfixing Trichodesmium in upper ocean nutrient cycles and primary productivity (Falkowski, 1997; Karl et. al., 1997; Capone et. al., 1997) has undescored the need to understand how this organism may utilize solid forms of iron to satisfy its high iron requirements, as has been proposed (Reuter et. al., 1992). The ${ }^{133} \mathrm{Ba}$ inert tracer method could be a valuable tool in studies of these types of processes, particularly when applied in combination with other techniques specific for reactive Fe. In the next chapter, the ${ }^{133} \mathrm{Ba}$ method is applied to studies of the biological dissolution of iron oxides, as mediated by phagotrophic protozoans. 


\section{References}

- Bishop, J.K.B. 1988. The barite-opal-organic carbon association in oceanic particulate matter. Nature. 332: 341-343.

- Bondietti, G., J. Sinninger and W. Stumm. 1993. The reactivity of Fe(III) (hydr)oxides: effects of ligands in inhibiting the dissolution. p. 157-167. In: Colloids and Surfaces A: Physicochemical and Engineering Aspects, 79. Elsevier Science Publishers B.V., Amsterdam.

- Bruland, K.W. 1983. Trace elements in seawater. p. 157-220. In Chemical Oceanography, Vol. 8. Riley, J.P and R. Chester, eds. Academic Press, London.

- Capone, D. G., Zehr, J.P., Paerl, H.W., Bergman, B. \& Carpenter, E.J. Trichodesmium, a globally significant marine cyanobacterium. Science 276,1221-1229 (1997).

- Cornell, R.M. and U. Schwertmann. 1996. The Iron Oxides: Structure, Properties, Reactions, Occurrence and Uses. VCH, New York, NY.

- Deng, B. and A.T. Stone. 1996. Surface-catalyzed chromium(VI) reduction: Reactivity comparisons of different organic reductants and different oxide surfaces. Environ. Sci. Technol. 30: 2484-2494.

- Dymond, J., E. Suess and M. Lyle. 1992. Barium in deep-sea sediment: A geochemical proxy for paleoproductivity. Paleoceanography 7: 163-181.

- Dzombek, D.A. and F.M.M. Morel. 1990. Surface Complexation Modeling: Hydrous Ferric Oxide. Wiley-Interscience. New York, NY.

- Eggleton, R. A. and R.W. Fitzpatrick. 1988. New data and a revised structural model for ferrihydrite. Clays \& Clay Minerals 36: 111-124.

- Elrod, V.A., K.S. Johnson and K.H. Coale. 1991. Determination of subnanomolar levels of iron (II) and total dissolved iron in seawater by flow injection analysis with chemiluminescence detection. Anal. Chem. 63: 893-898.

- Falkowski, P.G. Evolution of the nitrogen cycle and its influence on the biological sequestration of $\mathrm{CO}_{2}$ in the ocean. Nature 387, 272-275 (1997). 
- Gledhill, M. and C.M.G. van den Berg. 1994. Determination of complexation of iron(III) with natural organic complexing ligands in seawater using cathodic stripping voltammetry. Mar. Chem. 47: 41-54.

- Haygoood, M.G., P.D. Holt and A. Butler. 1993. Aerobactin production by a planktonic marine Vibrio sp. Limnol. Oceanogr. 38: 1091-1097.

- Johnson, K.S., K.H. Coale, V.A. Elrod and N.W. Tindale. 1994. Iron photochemistry in the equatorial Pacific. Mar. Chem. 46: 319-334.

- Johnson, K.S., R.M. Gordon and K.H. Coale. 1997. What controls dissolved iron concentrations in the world ocean? Mar. Chem. 57: 137-161.

- Karl, D., Letelier, R., Tupas, L., Dore, J., Christian, J. \& Hebel, D. The role of nitrogen fixation in biogeochemical cycling in the subtropical North Pacific Ocean. Nature 388, 533-538 (1997).

- King, D.W., H.A. Lounsbury and F.J. Millero. 1995. Rates and mechanism of Fe(II) oxidation at nanomolar total iron concentrations. Environ. Sci. Technol. 29: 818-824.

- King, D.W., J. Lin and D.R. Kester. 1991. Spectrophotometric determinations of Fe(II) in seawater at nanomolar concentrations. Anal. Chim. Acta 247: 125-132.

- Li, Y.-H., L. Burkhardt, M. Buchholtz, P. O'Hara and P.H. Santschi. 1984. Partition of radiotracers between suspended particles and seawater. Geochim. Cosmochim. Acta 48: 2011-2019.

- Martin, J.H., R.M. Gordon and S. Fitzwater. 1991. The case for iron. Limnol. Oceanogr. 36: 1793-1802.

- Measures, C.I., J. Yuan and J.A. Resing. 1995. Determination of iron in seawater by flow injection analysis using in-line preconcentration and spectrophotometric detection. Mar. Chem. 50: 3-12.

- Moffett, J.W. and O. C. Zafiriou. 1993. The photochemical decomposition of hydrogen peroxide in surface waters of the eastern Caribbean and Orinoco River. Journal Geophys. Res. 98: 2307-2313.

- Moffett, J.W. and R.G. Zika. 1987. Reaction kinetics of hydrogen peroxide with copper and iron in seawater. Env. Sci. Technol. 21: 804-810. 
- Nyffeler, U.P., Y.-H. Li and P.H. Santschi. 1984. A kinetic approach to describe traceelement distribution between particles and solution in natural aquatic systems. Geochim. Cosmochim. Acta 48: 1513-1522.

- O'Sullivan, D.W., A.K. Hanson and D.R. Kester. 1995. Stopped flow luminol chemiluminescence determination of $\mathrm{Fe}$ (II) and reducible iron in seawater at subnanomolar levels. Mar. Chem. 49: 65-77.

- O'Sullivan, D.W., A.K. Hanson, W. Miller and D.R. Kester. 1991. Measurement of $\mathrm{Fe}(\mathrm{II})$ in surface water of the equatorial Pacific. Limnol. Oceanogr. 36: 1727-1741.

- Obata, H. 1993. Automated determination of iron in seawater by chelating resin concentration and chemiluminescence detection. Anal. Chem. 65: 1524-1528.

- Rich, H.W. and F.M.M. Morel. 1990. Availability of well-defined iron colloids to the marine diatom Thalassiosira weissflogii. Limnol. Oceanogr. 35: 652-662.

- Rue, E.L. and K. W. Bruland. 1995. Complexation of iron(III) by natural organic ligands in the Central North Pacific as determined by a new competitive ligand equilibration/adsorptive cathodic stripping voltammetric method. Mar. Chem. 50: 117138.

- Schwertmann, U. and R.M. Cornell. 1991. Iron Oxides in the Laboratory. VCH, New York, NY.

- Suter, D., S. Banwart and W. Stumm. 1991. Dissolution of hydrous iron (III) oxides by reductive mechanisms. Langmuir 7: 809-813.

- Voelker, B.M. and D.L. Sedlak. 1995. Iron reduction by photoproduced superoxide in seawater. Mar. Chem. 50: 93-102.

- Waite, T.D. and F.M.M. Morel. 1984a. Photoreductive dissolution of colloidal iron oxide: Effect of citrate. J. Colloid. Interface Sci. 102:121-137.

- Waite, T.D. and F.M.M. Morel. 1984b. Photoreductive dissolution of colloidal iron oxides in natural waters. Environ. Sci. Technol. 18: 860-868.

- Waite, T.D. and F.M.M. Morel. 1984c. Coulometric study of the redox dynamics of iron in seawater. Anal. Chem. 56: 787-792. 
- Wells, M.L. and L.M. Mayer. 1991a. The photoconversion of colloidal iron hydroxides in seawater. Deep-Sea Res. 38: 1379-1395.

- Wells, M.L. and L.M. Mayer. 1991b. Variations in the chemical lability of iron in estuarine, coastal and shelf waters and its implications for phytoplankton. Mar. Chem. 32: $195-210$.

- Wells, M.L., L.M. Mayer and Guillard, R.R.L. 1991a. A chemical method for estimating the availability of iron to phytoplankton in seawater. Mar. Chem. 33: 23-40.

- Wells, M.L., L.M. Mayer, O.F.X. Donard, M.M. de Souza Sierra and S. Ackleson. 1991b. The photolysis of colloidal iron in the oceans. Nature 353: 248-250.

- Wu, J. and G.W. Luther. 1995. Complexation of Fe(III) by natural organic ligands in the Northwest Atlantic Ocean by a competitive ligand equilibration method and kinetic approach. Mar. Chem. 50: 159-177.

- Zepp, R.G., B.C. Faust and J. Hoigne. 1992. Hydroxyl radical formation in aqueous reactions ( $\mathrm{pH} 3-8$ ) of iron (II) with hydrogen peroxide: The photo-Fenton reaction. Env. Sci. Technol. 26: 313-319. 


\section{CHAPTER 5}

\section{Dissolution of colloidal iron oxides by protozoan grazers: \\ Measurement of absolute dissolution rates in the laboratory and in the field}

\section{Introduction}

Previous work - the need for a new technique

In Chapter 3 of this thesis, a number of independent methods were used to demonstrate in laboratory model systems that protozoan grazers can cause the dissolution of colloidal ferrihydrite. Having demonstrated the existence of a novel, grazer-mediated pathway for iron oxide dissolution in oxic systems, the next research objectives were to make a quantitative determination of the rate of this process, and to demonstrate its occurrence in the field. In order to accomplish these objectives, previous results indicated that it would be necessary to develop a methodology uniquely suited to the task. For various reasons, none of the techniques used in Chapter 3 are applicable to rate measurements or field studies.

Competitive ligand exchange techniques were successfully employed to demonstrate an increase in the chemical reactivity of iron oxides in the presence of actively grazing protozoans. Methods used included extraction of oxine- ${ }^{59} \mathrm{Fe}$ complexes by $\mathrm{C}-18$ chromatography (Wells et. al., 1991a) and measurement of Fe complexation with 1nitroso-2-napthol by competitive stripping voltammetry (Wu and Luther, 1995). Results with both methods indicated a peak in reactive iron in culture systems within a fairly brief window when concentrations of both protists and bacteria were relatively high, and grazing was most intense (see Figures 3.3 and 3.6). These data indicate that, outside of that window, removal of reactive iron due to hydrolysis/polymerization reactions occurs on timescales similar to the formation of reactive iron via protistan grazing. Competitive ligand exchange methods only measure a net rate of accumulation of reactive iron, not the actual rate at which colloidal ferrihydrite is being dissolved/reworked by protozoan grazers. In a field experiment, at much lower concentrations of grazers, the rate of grazer- 
mediated dissolution of iron oxides would be low enough that no accumulation of reactive iron would be measurable by competitive ligand exchange methods.

An additional analytical technique employed in Chapter 3 was size fractionation of ferrihydrite colloids radiolabeled with ${ }^{59} \mathrm{Fe}$ in model systems containing high concentrations of EDTA as a dissolved Fe trap (see Figure 3.3). By trapping ${ }^{59} \mathrm{Fe}$ in the dissolved phase, EDTA provides a signal that is essentially irreversible - there is a continual accumulation of dissolved ${ }^{59} \mathrm{Fe}$ over the course of the experiment. While the rate of accumulation of dissolved EDTA-complexed ${ }^{59} \mathrm{Fe}$ is different from a net reaction rate as obtained with competitive ligand exchange methods, it is still not easy to relate directly to the rate of production of reactive iron by protists. When EDTA is used as an iron trap, the rate of accumulation of dissolved ${ }^{59} \mathrm{Fe}$ is a function not only of the rate of dissolution of iron oxides by grazers, but is also a function of the kinetics of Fe-EDTA complexation, and of the thermal dissolution rate of ferrihydrite, which is likely to be elevated in the presence of high concentrations of EDTA (Rich and Morel, 1990). Adding a different chelator as a dissolved iron trap might reduce the kinetics problem, but would probably also increase the rate of thermal dissolution. Any attempt to measure the rate of dissolution of ferrihydrite by protists using this method would thus be operationallydefined.

The growth and chl $a$ per cell of iron-limited diatoms in model systems with and without ferrihydrite colloids and protozoan grazers was also used in Chapter 3 to demonstrate production of bioavailable iron from iron oxides by protists (see Figure 3.11). This technique proved to be fairly reproducible and quite sensitive under the conditions employed in our experiments. The diatoms apparently sequestered bioavailable iron produced over the course of the active grazing period of the cultures, and then demonstrated its effects by their increased growth rate and chl $a$ concentration. In this way, the diatoms provided an "integrated signal" of multiple dissolution events that occurred during the grazing period. Although a sensitive indicator, this integrated signal is very difficult to translate into a ferrihydrite dissolution rate because it is "filtered" through diatom physiology. Diatoms would also be very difficult to use as a 
probe for bioavailable iron in a field experiment, because factors like predation, competition, and nutrient status would be difficult to control.

\section{Development of a new method}

Based on the specific needs of this investigation and drawing conceptually from the techniques used in Chapter 3, a novel method of following the dissolution of iron oxides in seawater was designed. Ideally, this tool would have properties similar to the EDTAtrap or iron-limited diatom probes described above - the signal of iron oxide dissolution by protists would be additive and irreversible, independent of the continuous reverse reactions (hydrolysis and maturation) of the iron oxide. This would allow detection of protozoan-mediated dissolution of iron oxides, even at very low specific rates in field samples. Unlike the EDTA and diatom methods, however, this technique would enable a direct derivation of the rate and extent of protozoan-mediated dissolution of iron oxides, based on purely chemical principles, without complex kinetics.

Chapter 4 describes the development of this new method, based on the synthesis of colloidal ferrihydrite impregnated with the radiotracer ${ }^{133} \mathrm{Ba}$. ${ }^{133} \mathrm{Ba}$ is uniformly incorporated throughout the iron oxide, as shown by co-dissolution experiments. Upon dissolution/reaction of the iron oxide phase in seawater, ${ }^{133} \mathrm{Ba}$ is released from the oxide matrix. The very low affinity of $\mathrm{Ba}^{2+}$ for the iron oxide surface at seawater $\mathrm{pH}$, combined with the presence of high concentrations of similar alkali-earth cations $\mathrm{Mg}^{2+}$ and $\mathrm{Ca}^{2+}$ in seawater, causes ${ }^{133} \mathrm{Ba}$ to be released from the iron oxide very readily. Once released, ${ }^{133} \mathrm{Ba}$ remains in the dissolved phase due to its general lack of particle reactivity and involvement in biological metabolism. Thus, like the EDTA and diatom methods, the accumulation of ${ }^{133} \mathrm{Ba}$ in the dissolved phase provides an "integrated" signal of iron oxide dissolution, with the additional advantage that the rate of dissolved ${ }^{133} \mathrm{Ba}$ accumulation is directly related to the rate of iron oxide dissolution/alteration.

In Chapter 4 the ${ }^{133} \mathrm{Ba}$ technique was applied to studies of iron oxide dissolution by both photochemical and ligand/thermal mechanisms. Results from photochemical experiments under different conditions of $\mathrm{pH}$, light intensity, and chromophore 
concentration are in good agreement with previous studies of iron oxide photochemistry in seawater (Waite and Morel 1984a,b; Wells and Mayer 1991a). Ligand/thermal dissolution data indicate the potential of the ${ }^{133} \mathrm{Ba}$ method for studies of relatively slow iron oxide reactions.

Application of new method to studies of grazer-mediated iron oxide dissolution

Having identified and developed a suitable method, the purpose of the work in this chapter is to apply this technique to studies of the dissolution of colloidal iron oxides by phagotrophic protists. In the first part of the experimental section, a number of grazing experiments with the ${ }^{133} \mathrm{Ba}$ method are presented, involving laboratory cultures of protozoan grazers. The first experiment in the series serves to demonstrate the basic performance of the technique in grazing studies by comparing production of dissolved ${ }^{133} \mathrm{Ba}$ and ${ }^{59} \mathrm{Fe}$ from co-labeled ferrihydrite in model systems both with and without added EDTA.

In the next experiment, the ${ }^{133} \mathrm{Ba}$ method is used to calculate the rate and efficiency of colloidal ferrihydrite dissolution in a protozoan grazing culture by equating the consumption of bacteria with the ingestion of colloidal ferrihydrite and the production of dissolved ${ }^{133} \mathrm{Ba}$ over the exponential growth phase of the culture. In additional laboratory experiments, the ${ }^{133} \mathrm{Ba}$ method is used to compare the relative ability of different protozoan species to dissolve colloidal ferrihydrite, and to examine the possible significance of interactions between protozoan grazing and photochemistry in the dissolution of iron oxides.

The second part of the experimental section presents the results of a series of field experiments. For this work freshly-collected Vineyard Sound water was size-fractionated, spiked with ${ }^{133} \mathrm{Ba}$-impregnated colloidal ferrihydrite, and incubated under a variety of conditions. The rate and efficiency of colloidal iron oxide dissolution by the natural grazing consortia was determined. The chapter concludes with a general discussion of the results of the laboratory and field rate measurements presented here. 


\section{Experimental}

Laboratory experiments with protozoan grazers in culture

${ }^{133} \mathrm{Ba}$-impregnated colloidal ferrihydrite synthesized and rinsed via dialysis as described in Chapter 3 was used in all of the laboratory and field experiments described in this chapter. Briefly, ${ }^{133} \mathrm{Ba}$ and ${ }^{59} \mathrm{Fe}$ were co-precipitated with iron oxide as a 2-line ferrihydrite by neutralization of an iron salt solution, then heated $\left(5 \mathrm{~min}\right.$.) at $90^{\circ} \mathrm{C}$. Excess ${ }^{133} \mathrm{Ba}$ was rinsed out of this phase by dialysis at $\mathrm{pH} 5$ for 60 hours, and colloids were used in experiments at 1-2 $\mu \mathrm{M} \mathrm{Fe}$ concentration. A fresh batch of colloidal ferrihydrite was synthesized for each grazing experiment.

${ }^{133} \mathrm{Ba} /{ }^{59} \mathrm{Fe}$ co-labeled ferrihydrite: grazer-mediated dissolution with/without EDTA - In order to demonstrate the basic performance of ${ }^{133} \mathrm{Ba}$-impregnated colloidal ferrihydrite as a tracer for grazer-mediated dissolution of iron oxides, production of dissolved ${ }^{133} \mathrm{Ba}$ and dissolved ${ }^{59} \mathrm{Fe}$ from co-labeled colloids by grazers was compared in model systems both with and without EDTA added as a trap for dissolved Fe. Controls consisted of colloidal ferrihydrite added to $0.2 \mu \mathrm{m}$-filtered Vineyard Sound seawater (VSW). Grazing cultures contained $H$. halodurans as the bacterial prey and the microflagellate Cafeteria as the grazer. The following model systems were set up for this experiment:

- VSW with ${ }^{133} \mathrm{Ba} /{ }^{59} \mathrm{Fe}$ ferrihydrite (2)

- VSW with ${ }^{133} \mathrm{Ba} /{ }^{59} \mathrm{Fe}$ ferrihydrite and $10 \mu \mathrm{M}$ EDTA (2)

- VSW with ${ }^{133} \mathrm{Ba} /{ }^{59} \mathrm{Fe}$ ferrihydrite, $H$. halodurans, and Cafeteria (2)

- VSW with ${ }^{133} \mathrm{Ba} /{ }^{59} \mathrm{Fe}$ ferrihydrite, $H$. halodurans, Cafeteria, and $10 \mu \mathrm{M}$ EDTA (2)

Ferrihydrite was added to all model systems at $\sim 2 \mu \mathrm{M}$ Fe concentration.

$H$. halodurans was grown up for this experiment on yeast extract medium, harvested by centrifugation in lag phase, rinsed 3x, and resuspended in VSW in experimental bottles at $\sim 2.5 \times 10^{7}$ cells $/ \mathrm{ml}$. Cafeteria was inoculated from a log phase culture at $\mathrm{t}_{0}$, for an initial concentration of $\sim 10^{4}$ cells $/ \mathrm{ml}$. The volume of all model systems was $200 \mathrm{ml}$, in $250 \mathrm{ml}$ 
square polycarbonate bottles. Model systems were incubated without shaking at room temperature, under normal laboratory fluorescent lighting.

Instead of using filtration through $0.05 \mu \mathrm{m}$ pore-size Nuclepore membranes as described previously, dissolved ${ }^{133} \mathrm{Ba}$ and ${ }^{59} \mathrm{Fe}$ in this experiment were isolated by a dialysis technique. The advantage of dialysis for isolating dissolved ${ }^{133} \mathrm{Ba}$ in grazing experiments is that it is an extremely gentle method which does not cause grazer lysis. Sterile cellulose ester dispodialyzers (Spectrum, $3500 \mathrm{MWCO}, 5 \mathrm{ml}$ capacity) were filled with clean $0.2 \mu \mathrm{m}$-filtered VSW and added to each model system. The dispodialyzers were allowed to remain suspended in the model systems for about 24 hours, during which time dissolved ${ }^{133} \mathrm{Ba}$ and ${ }^{59} \mathrm{Fe}$ collected inside the bags by passive diffusion. To collect the dissolved tracers, the dispo-dialyzers were taken out of the model systems, their contents quantitatively removed by dispo-pipette, and weighed into small polypropylene Qorpak sample cups. For total samples, an equivalent weight of whole culture was removed at the same time and placed in an identical sample cup. Both dialysis solutions and totals were acidified with hydroxylamine hydrochloride solution to dissolve ferrihydrite in the totals and prevent wall loss in all samples. ${ }^{133} \mathrm{Ba}$ and ${ }^{59} \mathrm{Fe}$ activities were determined on a low-energy germanium detector. Values for dissolved ${ }^{133} \mathrm{Ba}$ obtained via dialysis methods are comparable to those obtained via $0.05 \mu \mathrm{m}$ filtration, although dialysis values are generally somewhat lower in dynamically changing systems.

The dialysis bags for these samples were added to the cultures 24 hours after $t_{0}\left(t_{0}\right.$ being the point at which the protistan inoculum was added to the grazing cultures) and removed 24 hours later at $t_{48}$. Results for these dialysis bag samples are shown in Figure 5.1. In this figure, the $\%$ of ${ }^{133} \mathrm{Ba}$ and ${ }^{59} \mathrm{Fe}$ collected in the $3500 \mathrm{MWCO}$ dialysis bags $(y$ axis) has been normalized to the total ${ }^{133} \mathrm{Ba}$ and ${ }^{59} \mathrm{Fe}$ activity in the culture at the time of dialysis bag collection. It can be seen that the grazer produced a dissolved ${ }^{133} \mathrm{Ba}$ signal of about $25 \%$ (relative to signal in the $0.2 \mu \mathrm{m}$ filtered control at $\sim 5 \%$ ) both with and without the presence of $10 \mu \mathrm{M}$ EDTA. The amount of dissolved ${ }^{133} \mathrm{Ba}$ produced by the grazing activity of Cafeteria was equivalent, within error, in cultures with and without EDTA. In contrast, much more dissolved ${ }^{59} \mathrm{Fe}$ was generated in grazing cultures with EDTA than 


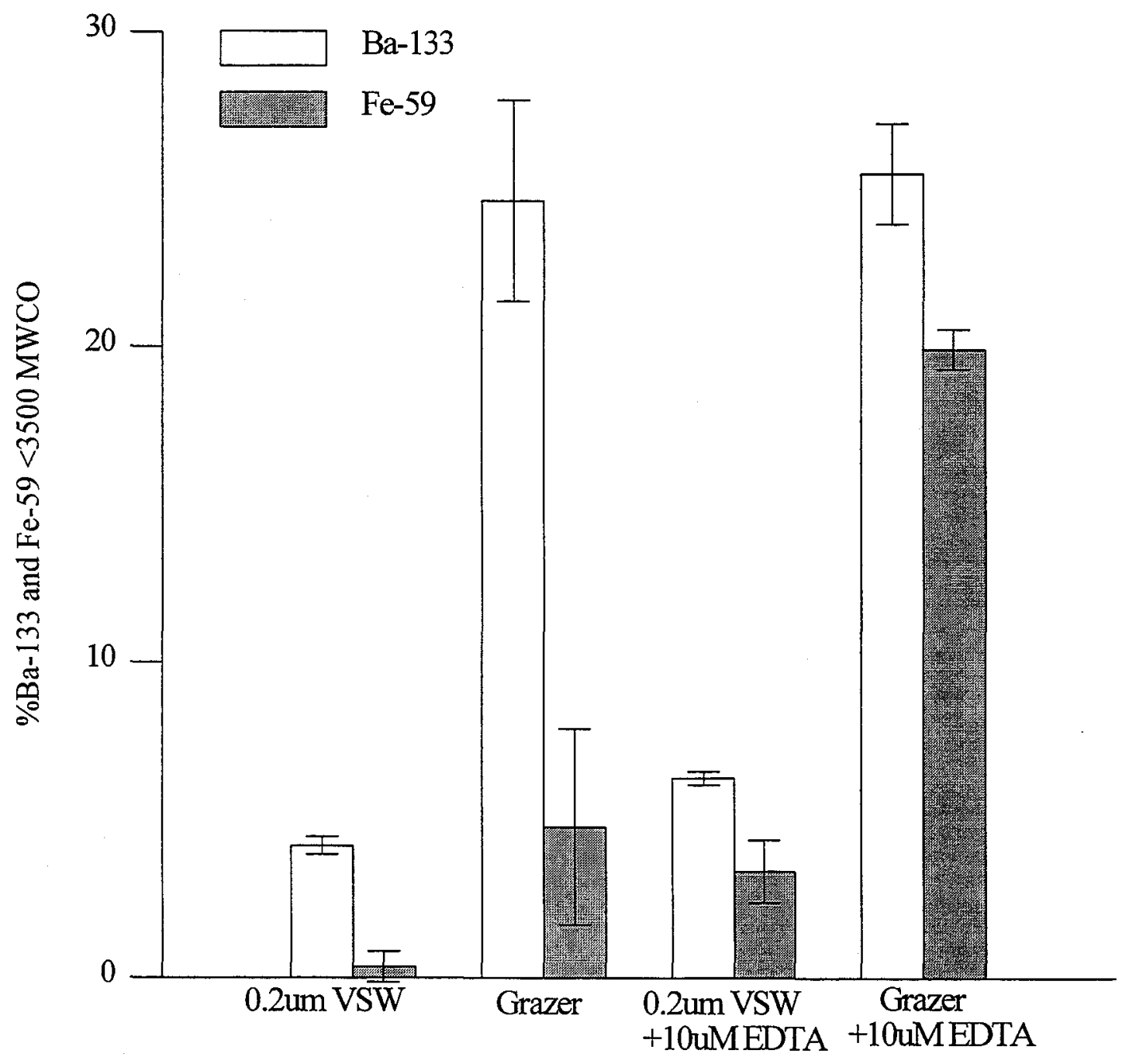

Figure 5.1 - Grazer-mediated dissolution of colloidal ferrihydrite as shown by production of dissolved $\mathrm{Ba}-133$ and $\mathrm{Fe}-59$ in model systems with and without EDTA, at $t=48$ hours. Grazing cultures (Cafeteria) vs. filtered VSW controls. 
without. This data demonstrates how ${ }^{133} \mathrm{Ba}$ was released from the colloids through grazing and accumulated in the dissolved phase, while ${ }^{59} \mathrm{Fe}$ was rapidly cycled back onto particle surfaces without building up a measurable dissolved concentration unless EDTA was present. In the filtered controls, $2-3 \%$ more dissolved ${ }^{59} \mathrm{Fe}$ and ${ }^{133} \mathrm{Ba}$ were observed in the presence of EDTA relative to the control without EDTA, indicating a small effect of the EDTA itself on colloidal Fe solubility.

These results demonstrate that, in the same way that grazers can cause an increase in the chemical and biological reactivity of iron oxides as shown in Chapter 3, grazers can also increase the rate of ${ }^{133} \mathrm{Ba}$ release from the colloidal ferrihydrite to the dissolved phase. As discussed in Chapter 3, this most likely occurs as a consequence of ingestion of the colloidal iron oxide by the grazers with subsequent digestive processing in the grazer food vacuole. ${ }^{133} \mathrm{Ba}$ release may occur within the vacuole itself, or it may also occur upon excretion of the chemically altered (and therefore more reactive) iron oxide back into the seawater medium by the protist. Most likely both processes are operative in increasing the accumulation of dissolved ${ }^{133} \mathrm{Ba}$ in grazing cultures. The cycling of ${ }^{133} \mathrm{Ba}$ and $\mathrm{Fe}$ is certainly de-coupled through the grazing process, due to reprecipitation of the $\mathrm{Fe}$ either during the latter stages of vacuole processing (when $\mathrm{pH}$ is near neutral) or upon excretion into seawater. Despite Fe re-precipitation reactions, ${ }^{133} \mathrm{Ba}$ should be effectively released due to its exceedingly low affinity for the iron oxide surface. Even if ${ }^{133} \mathrm{Ba}$ becomes re-associated with precipitated Fe oxide within the concentrated conditions inside the food vacuole, this ${ }^{133} \mathrm{Ba}$ should be effectively released from the amorphous, freshly-precipitated iron oxide surface upon excretion into seawater. (Some evidence for this comes from the high ${ }^{133} \mathrm{Ba}$ blanks associated with filtration/resuspension rinsed ${ }^{133} \mathrm{Ba}$-impregnated ferrihydrite, as described in $\mathrm{Ch}$. 4. These more labile $\mathrm{Fe}$ oxide phases continually leach ${ }^{133} \mathrm{Ba}$ into seawater.) Although the cycling of ${ }^{133} \mathrm{Ba}$ and $\mathrm{Fe}$ in the system is de-coupled, it is known from previous work in Chapter 3 (also from Fig. 5.1) that ${ }^{133} \mathrm{Ba}$ release through grazing coincides with an increase in the reactivity and bioavailability of the Fe itself. 
As mentioned above, the \% dissolved ${ }^{133} \mathrm{Ba}$ and ${ }^{59} \mathrm{Fe}$ in Figure 5.1 is normalized to total ${ }^{133} \mathrm{Ba}$ and ${ }^{59} \mathrm{Fe}$ activity in the cultures at $\mathrm{t}=48$ hours. Results indicate that wall losses in these model systems over 48 hours were about $30 \%$ for ${ }^{133} \mathrm{Ba}$ and $35 \%$ for ${ }^{59} \mathrm{Fe}$. Tests have shown that ${ }^{133} \mathrm{Ba}$ added to grazing cultures and controls in dissolved form is not lost to bottle walls or taken up by particles; therefore the wall loss in these experiments is interpreted as adhesion of colloidal ferrihydrite, with its associated ${ }^{59} \mathrm{Fe}$ and ${ }^{133} \mathrm{Ba}$, to bottle walls. Some additional wall loss of ${ }^{59} \mathrm{Fe}$ may occur in grazing cultures via scavenging of grazer-produced dissolved ${ }^{59} \mathrm{Fe}$ by bottle walls. The effect of wall loss causes an increase in the apparent percentage of dissolved ${ }^{133} \mathrm{Ba}$ and ${ }^{59} \mathrm{Fe}$ in model systems by decreasing total ${ }^{133} \mathrm{Ba}$ and ${ }^{59} \mathrm{Fe}$. For the data in Figure 5.1, the wall loss effect caused the apparent percentage of dissolved ${ }^{133} \mathrm{Ba}$ in grazing cultures to increase from about $17 \%$ to $25 \%$. For ${ }^{59} \mathrm{Fe}$, the wall loss effect increased the dissolved ${ }^{59} \mathrm{Fe}$ in the grazing culture with EDTA from about $10 \%$ to $20 \%$. The effect of wall loss on interpretation of the results of grazing experiments with ${ }^{133} \mathrm{Ba}$-impregnated colloidal ferrihydrite is considered in further detail in subsequent experiments. The data in Figure 5.1 is shown normalized only to the totals at $t=48$ in order to demonstrate the comparitive behavior of $\mathrm{Ba}$ and $\mathrm{Fe}$ in systems with and without EDTA, and the basic validity of the ${ }^{133} \mathrm{Ba}$ method as a tracer of grazer-mediated dissolution of colloidal iron oxides.

Rate and efficiency of grazer-mediated dissolution of colloidal iron oxides - In a subsequent grazing experiment, ${ }^{133} \mathrm{Ba}$-impregnated iron oxides were used to make a quantitative estimation of the rate and efficiency of colloidal ferrihydrite dissolution by Cafeteria. Protocols are similar to those described in the previous experiment, with some exceptions. The bacterial prey, $H$. halodurans, were grown up on yeast extract, heatkilled (incubation for 2 hours at $60^{\circ} \mathrm{C}$ ), and rinsed and resuspended $3 \mathrm{x}$ in sterile VSW prior to being used in the experiment. The following model systems were set up for this experiment, each $200 \mathrm{ml}$ in $250 \mathrm{ml}$ polycarbonate bottles:

- VSW with ${ }^{133} \mathrm{Ba} /{ }^{59} \mathrm{Fe}$ ferrihydrite and heat-killed H. halodurans (2)

- VSW with ${ }^{133} \mathrm{Ba} /{ }^{59} \mathrm{Fe}$ ferrihydrite, heat-killed H. halodurans, and Cafeteria (3) 
- VSW with heat-killed $H$. halodurans and Cafeteria (2) (non-labeled grazing cultures)

Appropriate model systems were inoculated with Cafeteria (from a culture growing on heat-killed $H$. halodurans) at $\mathrm{t}_{0}$. In order to estimate the rate and efficiency of colloidal ferrihydrite dissolution by Cafeteria, the rate of consumption of bacterial biomass by the grazer was used as a proxy for the rate of ingestion of colloidal ferrihydrite. The nonlabeled grazing cultures were used to obtain counts of bacteria and protozoans over the course of the experiment and thus determine the rate of consumption of bacterial biomass. Samples for counting were preserved in $1 \%$ glutaraldehyde and stored under refrigeration for 1-2 weeks before slides were prepared using the acridine orange staining methodology described in Chapter 2.

In order to ensure that the colloidal ferrihydrite was uniformly associated with bacterial biomass in the radio-labeled cultures, several hours prior to the start of the experiment heat killed $H$. halodurans and ${ }^{133} \mathrm{Ba} /{ }^{59} \mathrm{Fe}$ colloidal ferrihydrite were mixed together at a concentration of $3 \times 10^{8}$ cells $/ \mathrm{ml}$ bacteria and $10^{-5} \mathrm{M} \mathrm{Fe}$ as colloidal ferrihydrite in VSW. This mixture of colloids and bacteria in seawater was placed on a rotary shaker for several hours. Scavenging of the colloidal iron by bacterial cell surfaces was evidenced by the visible formation of orange flocs which settled to the bottom of the container when shaking stopped. Microscopic examination of the flocs formed during this procedure has confirmed that they are composed of a mixture of colloidal Fe oxides and bacteria. In order to use these coagulated bacteria in grazing experiments, mixed aliquots of the flocculated ferrihydrite/bacteria suspension are briefly ultra-sonicated (10 seconds) to break up the flocs, and then immediately added to VSW in the experimental bottles at $1 / 10$ dilution. At this lower concentration $\left(3 \times 10^{7}\right.$ cells $/ \mathrm{ml}$ bacteria and $1-2 \mu \mathrm{M}$ colloidal $\mathrm{Fe}$ ), the bacteria with surface-associated iron oxides form a stable suspension that can be used for grazing experiments.

This experiment was run over a period of 54 hours. Non-labeled grazing cultures were sampled for protist and bacteria counts throughout this time course. Results are shown in Figure 5.2A. The cultures containing ${ }^{133} \mathrm{Ba} /{ }^{59} \mathrm{Fe}$ ferrihydrite were sampled for dissolved 

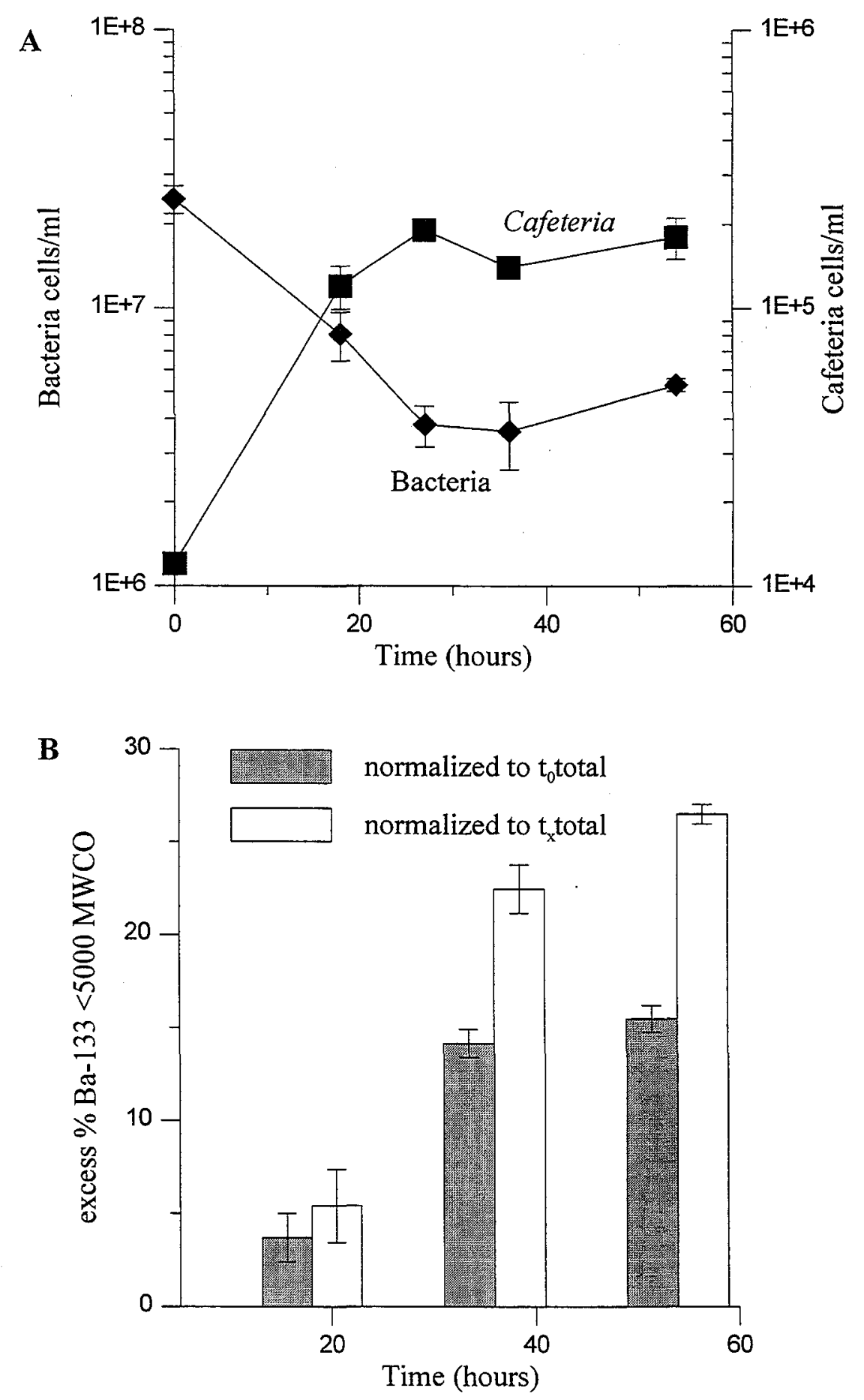

Figure 5.2 - Rate and efficiency of Cafeteria-mediated dissolution of iron oxide A - Population dynamics in grazing culture

$\mathrm{B}$ - Production of dissolved $\mathrm{Ba}-133$ in grazing culture relative to control 
${ }^{133} \mathrm{Ba}$ and ${ }^{59} \mathrm{Fe}$ using a dialysis technique similar to that described for the previous experiment. $5000 \mathrm{MWCO}$ dispo-dialyzers were used, incubated in model systems for 18 hours per sample. Three successive 18-hour dialysis samples were obtained over the 54 hour time course. The dialysis results are presented in Figure 5.2B, for ${ }^{133} \mathrm{Ba}<5000$ MWCO (since no EDTA was added, no dissolved ${ }^{59} \mathrm{Fe}$ was collected in dialysis bags in these model systems).

Dialysis results are presented as the excess $\%$ dissolved ${ }^{133} \mathrm{Ba}$ in grazing cultures (i.e. these values have been corrected for dissolved ${ }^{133} \mathrm{Ba}$ in the non-grazing bacterial controls, which averaged about $7-8 \%$ over the entire time course). Because significant (i.e. $>20 \%$ ) wall loss of both ${ }^{133} \mathrm{Ba}$ and ${ }^{59} \mathrm{Fe}$ occurred in the grazing cultures in this experiment, dialysis results are presented in two ways: 1 ) normalized to the total ${ }^{133} \mathrm{Ba}$ activity at $\mathrm{t}_{0}$ (grey bars); 2) normalized to the total ${ }^{133} \mathrm{Ba}$ activity at $\mathrm{t}_{\mathrm{x}}$ (white bars). Very little wall loss occurred in the non-grazing controls, so wall loss of colloidal ferrihydrite appears to be a function of the grazing process. Wall loss may occur due to the formation of surfaceactive waste products by the grazer. The formation of surface active materials as a consequence of grazing has been repeatedly observed, as evidenced by enhanced particle aggregation and accumulation of material at the air/water interface in grazing cultures. Surface active waste products may either be excreted by the protist already in association with the colloidal ferrihydrite, or they may scavenge iron oxides in bulk solution. In any case the association of colloidal ferrihydrite with surface active waste produced by the grazer may result in scavenging of the iron oxides by bottle walls.

Using the data in Figure 5.2, an estimate can be made of the rate and efficiency of colloidal iron oxide dissolution by Cafeteria in these cultures. In Figure 5.2A, bacterial consumption over the time period $t_{0}$ to $t_{27}$ can be simply modeled as exponential decay:

$$
(\text { cells } / \mathrm{ml})_{\mathrm{t}}=(\text { cells } / \mathrm{ml})_{t 0} \mathrm{e}^{-\mathrm{kt}}
$$

In these cultures, $\mathrm{k}=0.065 \mathrm{~h}^{-1}$, which can be recast as a specific grazing rate of $156 \% \mathrm{~d}^{-1}$.

If it is assumed that colloidal ferrihydrite is uniformly associated with the bacteria cells in the grazing culture, then ingestion of colloidal ferrihydrite follows the same rate 
expression. It is further assumed that the production of dissolved ${ }^{133} \mathrm{Ba}$ is proportional to the extent of dissolution/reaction of colloidal ferrihydrite. Therefore, the production of dissolved ${ }^{133} \mathrm{Ba}$ over the exponential growth period $\mathrm{t}_{0}-\mathrm{t}_{27}$ should give an indication of the extent of dissolution of the colloidal ferrihydrite ingested by the grazer over that same time period. The middle dialysis data point $\left(\mathrm{t}_{18}-\mathrm{t}_{36}\right)$ brackets the time period over which dissolved ${ }^{133} \mathrm{Ba}$ was produced by the culture in exponential growth. Taking the $\mathrm{t}_{0}$ normalized value as a minimum and the $t_{x}$-normalized value as a maximum, the data indicate that Cafeteria dissolved the colloidal iron oxide ingested during exponential growth with an efficiency of 17-26\%. Based on the rate constant derived above for ingestion of iron colloids over the same time period, this translates into a first-order rate constant for dissolution of colloidal ferrihydrite in these cultures of about 0.01-0.02 $\mathrm{h}^{-1}$. This is very close to the averaged rate constants calculated in Chapter 3 for the dissolution of colloidal ferrihydrite in natural waters during 1 hour irradiations with the $1000 \mathrm{~W} \mathrm{Hg} / \mathrm{Xe}$ lamp $\left(0.02-0.03 \mathrm{~h}^{-1}\right)$. This suggests that, as measured by production of dissolved ${ }^{133} \mathrm{Ba}$, the rate of dissolution of colloidal ferrihydrite in a concentrated, exponential growth-phase Cafeteria culture is similar to the rate of ferrihydrite dissolution under full-spectrum irradiation by a $1000 \mathrm{~W} \mathrm{Hg} / \mathrm{Xe}$ lamp.

Species Comparison Experiment - In order to determine the relative efficiency of several different cultured strains of protozoan grazers at dissolving colloidal iron oxides, a species comparison experiment was conducted using ${ }^{133} \mathrm{Ba}$-impregnated colloidal ferrihydrite. Protozoan species tested in this experiment included the following: Cafeteria sp. (clone Cflag), Uronema (clone BBcil), Pteridomonas (clone NB1), and a hymenostome ciliate (clone Hcil). All of these organisms were taken from the culture collection of Dave Caron. Bacterial prey for all grazers was H. halodurans, grown on glucose medium. Bacteria were harvested by centrifugation and rinsed three times in VSW prior to use in experiments, but were not heat-killed.

Replicate cultures of each grazer were set up with bacterial prey and ${ }^{133} \mathrm{Ba} /{ }^{59} \mathrm{Fe}$ labeled ferrihydrite, along with two control cultures containing only bacteria and labeled 
ferrihydrite. Additional replicate cultures of each grazer with bacteria and non-labeled ferrihydrite were also set up to sample for protist and bacteria cell counts. Non-labeled ferrihydrite was added to these cultures in order to ensure that bacterial ingestion rates would be similar to the systems containing radio-labeled ferrihydrite. Samples for cell counts were prepared using acridine orange staining. All model systems were $200 \mathrm{ml}$ in $250 \mathrm{ml}$ polycarbonate bottles, incubated at room temperature without agitation under normal laboratory lighting. As described in the previous experiment, ferrihydrite and bacteria were mixed together for several hours prior to the experiment in order to ensure uniform scavenging of the colloidal iron oxides by the bacteria cells. Identical procedures were followed for both radio-labeled and non-labeled ferrihydrite/bacteria suspensions. Bacteria cell concentrations in all cultures at to were $6.7 \times 10^{6} \pm 4.6 \times 10^{5} \mathrm{cells} / \mathrm{ml}$. Protozoan cell concentrations at t $t_{0}$ were as follows: Cafeteria $-1.5 \times 10^{4}$ cells $/ \mathrm{ml}$; Uronema - $5.8 \times 10^{2}$ cells $/ \mathrm{ml}$; Pteridomonas - $7.3 \times 10^{3}$ cells $/ \mathrm{ml}$; Hcil $-2.9 \times 10^{3}$ cells $/ \mathrm{ml}$.

Starting at $\mathrm{t}_{0}$, model systems were sampled every 10 hours for dissolved and total ${ }^{133} \mathrm{Ba}$ and ${ }^{59} \mathrm{Fe}$, and for cell counts. Uronema cultures were run for 40 hours; cultures of all other organisms were run for 50 hours. Instead of using dialysis, dissolved ${ }^{133} \mathrm{Ba}$ and ${ }^{59} \mathrm{Fe}$ was determined using the filtration technique described in Chapter 4 (syringefiltration through a $47 \mathrm{~mm}$ diameter, $0.05 \mu \mathrm{m}$ pore-size Nuclepore polycarbonate filter). This technique provides better time resolution than dialysis, since a discrete sample can be taken at any time point. Unfiltered aliquots were also taken for determination of total ${ }^{133} \mathrm{Ba}$ and ${ }^{59} \mathrm{Fe}$. All filtrate and total samples were weighed out to equal mass (about $10 \mathrm{~g}$ ) and stored in polypropylene sample cups of identical geometry for counting on our lowenergy germanium detector. An aliquot of hydroxylamine $\mathrm{HCl}$ solution $(250 \mu \mathrm{l})$ was added to all total and filtrate samples to dissolve colloidal iron and prevent wall loss.

Results are shown in Figure 5.3 for dissolved ${ }^{133} \mathrm{Ba}$ production in all grazing cultures plus the bacteria only control. As in the previous experiment, significant ( $>20 \%$ in some cases) wall loss occurred in the grazing cultures, but not in the bacteria only control. For this reason, results are presented both normalized to the ${ }^{133} \mathrm{Ba}$ total at $\mathrm{t}_{0}($ Fig. $5.3 \mathrm{~A}$ ) and normalized to the ${ }^{133} \mathrm{Ba}$ total at $\mathrm{t}_{\mathrm{x}}$ (Fig. 5.3 B). All species tested caused a measurable 

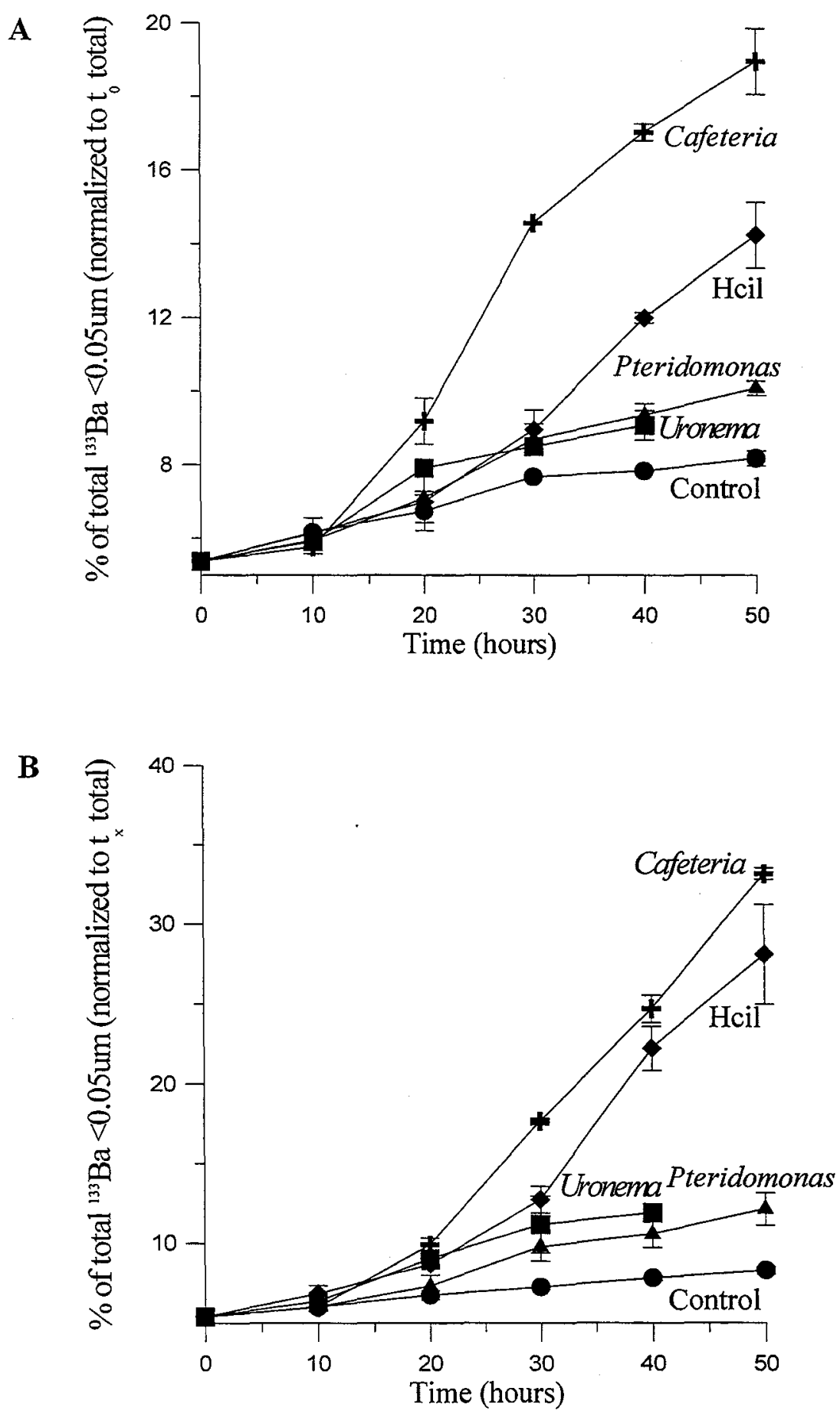

Figure 5.3 - Dissolution of colloidal ferrihydrite by different protozoan species A - \% dissolved Ba-133 vs time, normalized to Ba-133 total @ $\mathrm{t}=0$ B - \% dissolved Ba-133 vs time, normalized to Ba-133 total @ $\mathrm{t}=\mathrm{x}$ 
increase in the amount of dissolved ${ }^{133} \mathrm{Ba}$ produced in model systems, whether results are expressed as normalized to ${ }^{133} \mathrm{Ba}$ at $\mathrm{t}_{0}$ or at $\mathrm{t}_{\mathrm{x}}$. There was considerable variation, however, in the amount of dissolved ${ }^{133} \mathrm{Ba}$ produced by different organisms. Cafeteria and Hcil both appeared to be considerably more effective than Uronema and Pteridomonas at releasing ${ }^{133} \mathrm{Ba}$ from colloidal ferrihydrite.

For comparison of $\%$ dissolved ${ }^{133} \mathrm{Ba}$ with population dynamics in cultures of different grazers, Table 5.1 presents data on \% dissolved ${ }^{133} \mathrm{Ba}$ and protozoan and bacteria cell concentrations at $t_{48}$. There was no correlation between the $\%$ bacteria consumed and the excess $\%{ }^{133} \mathrm{Ba}$ dissolved by a given grazer. There was also no correlation between the cell yield for a given protozoan species and the amount of dissolved ${ }^{133} \mathrm{Ba}$ produced in culture. $\mathrm{pH}$ variations in these grazing cultures were not significant, and were not correlated with dissolved ${ }^{133} \mathrm{Ba}$ (data not shown). These results indicate that variations in the degree of colloidal ferrihydrite reaction in the different grazer cultures (as indicated by production of dissolved ${ }^{133} \mathrm{Ba}$ ) were most likely related to specific characteristics of protozzoan metabolism, such as digestive vacuole chemistry. In the last column in Table 5.1 , excess $\%{ }^{133} \mathrm{Ba}$ dissolved in each grazing culture is normalized to the $\%$ consumption of bacterial biomass (assuming that colloidal ferrihydrite was associated with bacteria cells and ingested at the same rate), in order to calculate a consumption-normalized ferrihydrite dissolution efficiency for each grazer. Based on this calculation, Cafeteria and Hcil were 5-10 times as efficient as Uronema and Pteridomonas at dissolving colloidal ferrihydrite. The dissolution efficiency for Cafeteria, $14 \%$, is close to the $t_{0^{-}}$ normalized dissolution efficiency calculated for this organism in the previous experiment, $17 \%$ (Fig. 5.2).

If the four organisms shown in Table 5.1 are taken as representative of a natural grazing consortium, then averaging their individual efficiencies together yields an average colloidal iron oxide dissolution efficiency of the natural grazing consortium of $8.2 \%$. In the marine environment, specific grazing rates for natural consortia have been measured ranging from $20 \%$ to $70 \% \mathrm{~d}^{-1}$ (Valiela, 1995; Capriulo, 1990). Assuming that colloidal iron oxides of a size similar to or associated with prey cells would be turned 
Table 5.1 - Species Comparison Grazing Experiment

\begin{tabular}{|c|c|c|c|c|}
\hline Species & $\begin{array}{l}{ }^{1} \text { Protozoan } \\
\text { Cell Yield } \\
\text { (cells/ml) }\end{array}$ & $\begin{array}{c}2 \% \text { Bacteria } \\
\text { Consumed }\end{array}$ & $\begin{array}{c}{ }^{3} \text { Excess \% } \\
{ }^{133} \text { Ba Dissolved }\end{array}$ & $\begin{array}{r}{ }^{4} \text { Consumption- } \\
\text { Normalized } \\
\text { Efficiency }\end{array}$ \\
\hline Cafeteria & $2.7 \pm 0.4 \times 10^{5}$ & $77 \pm 3$ & 10.8 & $14 \%$ \\
\hline Uronema & $8.9 \pm 1.6 \times 10^{3}$ & $95 \pm 3$ & 1.2 & $1.3 \%$ \\
\hline Hcil & $1.6 \pm 0.2 \times 10^{4}$ & ${ }^{5} 40 \pm 10$ & 6.1 & $15 \%$ \\
\hline Pteridomonas & $1.7 \pm 0.2 \times 10^{5}$ & $70 \pm 3$ & 1.9 & $2.7 \%$ \\
\hline
\end{tabular}

1 - Protozoan cell yield refers to the concentration of protozoan grazers at the end-point of the culture.

2 - \% bacteria consumed refers to the \% bacteria remaining at the end-point of the culture relative to the bacteria concentration at $t_{0}$.

3 - As in Figure 5.2B - Values corrected for the \% dissolved ${ }^{133} \mathrm{Ba}$ in bacteria-only controls and normalized to total ${ }^{133} \mathrm{Ba}$ at $\mathrm{t}_{0}$.

4 - Consumption-normalized efficiency refers to the excess $\%{ }^{133} \mathrm{Ba}$ dissolved for each species, divided by the \% consumption of bacterial biomass (and by association, colloidal ferrihydrite) for each species.

5 - Error on this number is higher for Hcil due to cryptic growth of bacterial prey during the experiment. 

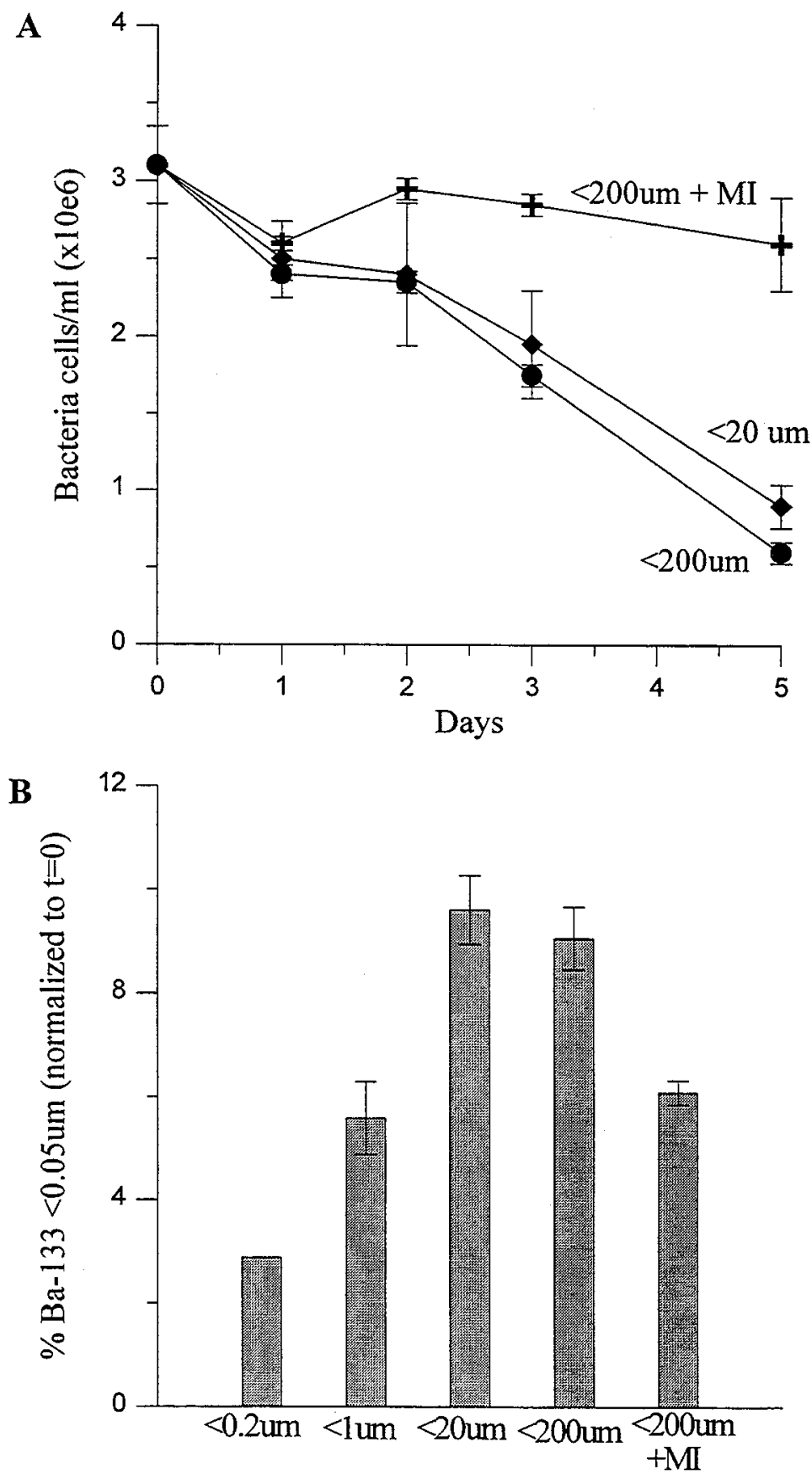

Figure 5.6 - Results from Field experiment \#1

A: Consumption of DTAF-stained bacteria in different treatments. $\mathrm{MI}=$ metabolic inhibitors

B: Production of dissolved Ba-133: 0.05um filter data at day 5, normalized to total $\mathrm{Ba}-133$ at $\mathrm{t}=0$. No replicate for $<0.2 \mathrm{um}$ size class. 
bacteria $\left(3 \times 10^{6}\right.$ cells $\left./ \mathrm{ml}\right)$ in this experiment was about 1 to 1.5 times as large as the naturally-occuring heterotrophic bacterial population in Vineyard Sound $\left(\sim 2.4 \times 10^{6}\right.$ cells/ml; Caron et. al., 1991).

Interpretation of the data for dissolved ${ }^{133} \mathrm{Ba}$ in this field experiment (and in the two other field experiments described in this chapter) is complicated by the issue of wall loss. As in the laboratory experiments described in the previous experimental section, wall loss of both ${ }^{133} \mathrm{Ba}$ and ${ }^{59} \mathrm{Fe}$ occurred in the actively grazing treatments, especially in the $<200 \mu \mathrm{m}$ size class, where wall loss was about $40 \%$. This loss of acitvity is attributed to scavenging of colloidal ferrihydrite containing both ${ }^{133} \mathrm{Ba}$ and ${ }^{59} \mathrm{Fe}$ by bottle walls. The results of the incubation of the two $<200 \mu \mathrm{m}$ samples spiked with dissolved ${ }^{133} \mathrm{Ba}$ indicate that no loss of ${ }^{133} \mathrm{Ba}$ occurred to the bottle walls between day 1 and day 5 (data not shown). At the end of the experiment, only $0.05 \%$ of the total ${ }^{133} \mathrm{Ba}$ activity was associated with particles caught by the $0.2 \mu \mathrm{m}$ Millipore filter. Thus, even over a relatively long incubation of 5 days, ${ }^{133} \mathrm{Ba}$ remained stable in solution.

As described previously for the laboratory experiments, loss of ${ }^{133} \mathrm{Ba}$ activity to the bottle wall increases the apparent percentage of dissolved ${ }^{133} \mathrm{Ba}$ produced by reducing total ${ }^{133} \mathrm{Ba}$ activity. Wall loss also acts to reduce the potential for production of dissolved ${ }^{133} \mathrm{Ba}$ in grazing cultures, however, by decreasing the amount of ${ }^{133} \mathrm{Ba}$-impregnated colloidal iron available for grazer consumption. Presenting \% dissolved ${ }^{133} \mathrm{Ba}$ data as normalized to ${ }^{133} \mathrm{Ba}$ activity at $\mathrm{t}_{0}$ can thus be thought of as a minimum estimate of the extent of colloidal iron oxide dissolution, while normalizing dissolved ${ }^{133} \mathrm{Ba}$ to total ${ }^{133} \mathrm{Ba}$ activity at $t_{t}$ is a maximum. This issue will be considered in more detail in the data for field experiments \#2 and \#3. Results for dissolved ${ }^{133} \mathrm{Ba}$ in field experiment \#1 are shown normalized to ${ }^{133} \mathrm{Ba}$ activity at $\mathrm{t}_{0}$ (Fig. 5.6B), which is the most conservative approach.

Dialysis data from $t_{48}-t_{72}$ was not usable because both $5000 \mathrm{MWCO}$ and $3500 \mathrm{MWCO}$ dispo-dialyzers were used (due to unavoidable circumstance), and results from the two different molecular weight cut-offs were not comparable because of differences in their diffusion/equilibration timescales. Dialysis data from $t_{0}-t_{24}$ and from $t_{24}-t_{48}$ (all obtained with 5000 MWCO dialysis bags) did not reveal any significant differences in the amount 
of dissolved ${ }^{133} \mathrm{Ba}$ produced in different incubation treatments as normalized to $\mathrm{t}_{0}$ (data not shown). The results of the $0.05 \mu \mathrm{m}$ filtrations performed on day 5 of the incubation, shown in Figure 5.6B, did indicate some significant differences in the amount of dissolved ${ }^{133} \mathrm{Ba}$ produced in different treatments. (There is no replicate for the $<0.2 \mu \mathrm{m}$ size fraction, because one of the $0.2 \mu \mathrm{m}$ incubations was mistakenly acidified during setup by passing the seawater through the $0.2 \mu \mathrm{m}$ filter cartridge before it was completely rinsed out after acid-washing.)

As Figure 5.6B indicates, dissolved ${ }^{133} \mathrm{Ba}$ in the actively grazing treatments exceeded that in the $<1 \mu \mathrm{m}$ and metabolically inhibited controls by about a factor of 1.6 . This data suggests that the mechanism for release of ${ }^{133} \mathrm{Ba}$ to the dissolved phase was via grazermediated dissolution of colloidal ferrihydrite. Incubations were carried out primarily in darkness, which would inhibit the activity of photosynthetic eukaryotes in the system. Therefore it is unlikely that colloidal iron oxide dissolution occurred as a consequence of the activity of photosynthetic eukaryotes. ${ }^{133} \mathrm{Ba}$ release in the $<1 \mu \mathrm{m}$ and metabolically inhibited $<200 \mu \mathrm{m}$ treatments was about the same. This is consistent with the fact that both of these treatments contained primarily prokaryotes as the metabolically active organisms. The difference in \% dissolved ${ }^{133} \mathrm{Ba}$ between these treatments and the $<0.2 \mu \mathrm{m}$-filtered treatment may have been due to some solubilization of colloidal ferrihydrite by prokaryotes. Alternatively, the dissolution could have been due to some residual grazing activity by inhibitor-resistant organisms or small flagellates that could have slipped through the $1 \mu \mathrm{m}$ filter. Interpretation of controls vs experimental data will be considered in more detail in field experiment \#3, where a full time course of dissolved and total ${ }^{133} \mathrm{Ba}$ was measured.

Field experiment \#2 - Protocols - Several changes were made in experimental protocols between field experiment $\# 1$ and $\# 2$. Incubations in field experiment $\# 2$ were carried out in larger (one liter) polycarbonate bottles, filled to $500 \mathrm{ml}$ in order to provide more headspace. A lower concentration of bacteria was added in association with the colloidal iron oxides $\left(0.6 \times 10^{6} \mathrm{cells} / \mathrm{ml}\right)$, in an attempt to reduce the turnover time of bacterial 
biomass (and associated iron oxides) in the system. Thirdly, incubations were conducted under constant light instead of darkness to preserve the activity of photosynthetic organisms in the system. In other respects, protocols in field experiment $\# 2$ were similar to those followed in field experiment $\# 1$.

Seawater for field experiment $\# 2$ was collected on $8 / 29 / 97$, at 4:45 pm from the WHOI dock on an incoming tide. Size fractions and treatments were set up as shown in Table 5.2, in 1 liter polycarbonate bottles. Heat-killed bacteria were added to both radiolabeled and non-labeled incubation bottles at a concentration of $0.6 \times 10^{6}$ cells $/ \mathrm{ml} .{ }^{133} \mathrm{Ba}-$ colloidal ferrihydrite was added at a concentration of $1-2 \mu \mathrm{M}$ Fe to radio-labeled incubations, in association with bacteria cells. After size fractions and treatments were set up, bottles were incubated under constant light (fluorescent ceiling lights) at $20^{\circ}$ to $22^{\circ} \mathrm{C}$, without agitation. Bottles were incubated for 5 days (120 hours) and sampled every 18 hours for cell counts and dissolved/total ${ }^{133} \mathrm{Ba}$. Dissolved ${ }^{133} \mathrm{Ba}$ samples were obtained by syringe filtration through a $0.05 \mu \mathrm{m}$ Nuclepore filter, as described previously.

Field experiment \#2 - Results - On day 5, all of the incubation treatments had percent dissolved ${ }^{133} \mathrm{Ba}$ values of between 4 and $6 \%$ (normalized to ${ }^{133} \mathrm{Ba}$ total activity at $\mathrm{t}_{0}$ ). Examination of the concentration of DTAF-stained bacteria in the non-labeled $<200 \mu \mathrm{m}$, $<20 \mu \mathrm{m}$, and $<200 \mu \mathrm{m}+$ metabolic inhibitor treatments on day 5 show that there was significant removal of DTAF-stained bacteria in the non-inhibited treatments relative to the inhibited treatment. This indicates that grazers were active. As in field experiment \#1, there was significant wall loss of ${ }^{133} \mathrm{Ba}$ and ${ }^{59} \mathrm{Fe}$ in the $<200 \mu \mathrm{m}$ ( $\sim 50 \%$ wall loss) and $<20 \mu \mathrm{m}$ ( $\sim 80 \%$ wall loss) treatments relative to $<1 \mu \mathrm{m}$ and $<0.2 \mu \mathrm{m}$ controls $(\sim 10 \%$ wall loss) by day 5 . Since wall loss of colloidal $\mathrm{Fe}$ is usually associated with grazing, this indicates that grazers consumed the ${ }^{133} \mathrm{Ba}$-impregnated ferrihydrite in the radio-labeled cultures.

The high percent wall loss in the grazing treatments in these experiments may explain the apparent lack of a grazing effect when dissolved ${ }^{133} \mathrm{Ba}$ in all treatments is normalized to total ${ }^{133} \mathrm{Ba}$ activity at $\mathrm{t}_{0}$. For example, if the dissolved ${ }^{133} \mathrm{Ba}$ activity in each incubation 
treatment at day 5 was normalized to the total ${ }^{133} \mathrm{Ba}$ activity present in each incubation treatment at that time point, then $\%$ dissolved ${ }^{133} \mathrm{Ba}$ in each incubation treatment would be as follows:

$$
\begin{gathered}
<200 \mu \mathrm{m}-9.4 \% \\
<20 \mu \mathrm{m}-28.4 \% \\
<200 \mu \mathrm{m}+\mathrm{MI}-7.7 \% \\
<1 \mu \mathrm{m}-4.7 \% \\
<0.2 \mu \mathrm{m}-5.4 \%
\end{gathered}
$$

When presented in this way, the data appear to clearly indicate a large grazing effect in the $<20 \mu \mathrm{m}$ size fraction. Yet when normalized to ${ }^{133} \mathrm{Ba}$ activity at $\mathrm{t}_{0}$, the grazing effect in the $<20 \mu \mathrm{m}$ size fraction at day 5 appears much smaller (dissolved ${ }^{133} \mathrm{Ba}$ in $<20 \mu \mathrm{m}$ of $6 \%$, vs $4.5 \%$ in non-grazing controls.) It may be that removal of grazer-available ${ }^{133} \mathrm{Ba}$ impregnated ferrihydrite in the $<20 \mu \mathrm{m}$ size class reduced the production of dissolved ${ }^{133} \mathrm{Ba}$ in that treatment relative to total ${ }^{133} \mathrm{Ba}$ activity at $\mathrm{t}_{0}$. Or, it may be that grazer effects in that size class led to $80 \%$ wall loss, without significant dissolution and production of dissolved ${ }^{133} \mathrm{Ba}$. It is difficult to say. It may be best to regard the results of this experiment as a range, with grazing effects in the $<20 \mu \mathrm{m}$ size fraction resulting in a minimum of $1.5 \%$ dissolution to a maximum of $20 \%$ dissolution relative to non-grazing controls. The range of grazing effects in the $<200 \mu \mathrm{m}$ size fraction was much less, from a fraction of a percent to $3.5 \%$ vs. non-grazing controls.

In general, the results of this experiment indicate less of an effect of grazing on colloidal iron oxide reactivity than was observed in field experiment \#1, where significant increases in dissolved ${ }^{133} \mathrm{Ba}$ were observed in both of the actively grazing incubations relative to the non-grazing controls, even when normalized to total ${ }^{133} \mathrm{Ba}$ activity at $t_{0}$. This may be due to qualitative differences in the composition of the natural grazing consortia in each experiment. Examination of DAPI-stained slides of the natural consortium in the $<200 \mu \mathrm{m}$ size fraction of the seawater used in field experiment \#2 indicate that the sample was relatively productive, containing a number of chain-forming 
diatoms similar in form to Chaetoceros as well as some other species. Some cells which appeared to be heterotrophic flagellates were observed. Relative to the natural consortium in field experiment \#1, there was a greater abundance of eukaryotic phytoplankton in field experiment \#2. Phytoplankton populations increased substantially in field experiment $\# 2$ by day 5 of the incubation in the $<200 \mu \mathrm{m}$, non-inhibited treatments.

Field experiment \#3 - Protocols - The final field experiment in this series, field experiment \#3, included an additional set of radio-labeled incubations (see Table 5.3). In addition to the series of radio-labeled incubations of ${ }^{133} \mathrm{Ba} /{ }^{59} \mathrm{Fe}$ colloidal ferrihydrite added in association with heat-killed bacteria (series 1 in Table 5.3), this experiment also included a series of radio-labeled incubations containing ${ }^{133} \mathrm{Ba} /{ }^{59} \mathrm{Fe}$ ferrihydrite added without bacteria (series 2). As in field experiments \#1 and \#2, the non-labeled incubations contained only DTAF-stained bacteria. Bacteria were added to both the nonlabeled incubations and the Series 1 radio-labeled incubations at a concentration of $\sim 3.8$ $\times 10^{6}$ cells $/ \mathrm{ml}$.

Vineyard Sound seawater for field experiment \#3 was collected at 9am on 9/18 on an incoming tide at the WHOI dock. Water temperature at the time of collection was $21^{\circ} \mathrm{C}$. Seawater was size-fractionated and various treatments set up as described previously, in 1 liter polycarbonate bottles filled to $500 \mathrm{ml}$ as in field experiment \#2. In addition to the incubations shown in Table 5.3, four additional incubations of $125 \mathrm{ml}$ each were set up in $250 \mathrm{ml}$ square polycarbonate bottles: two $<200 \mu \mathrm{m}$ and two $<20 \mu \mathrm{m}$. All four of these smaller cultures were spiked with dissolved ${ }^{133} \mathrm{Ba}$ to monitor ${ }^{133} \mathrm{Ba}$ wall loss and particle uptake. All bottles were incubated for 5 days at a temperature between $18^{\circ} \mathrm{C}$ and $20^{\circ} \mathrm{C}$, on a 12-hour light/12-hour dark cycle. Starting at $t_{0}, 1$ liter radio-labeled bottles were sampled every 24 hours for dissolved $\left(0.05 \mu \mathrm{m}\right.$ syringe filtration) and total ${ }^{133} \mathrm{Ba}$, and non-labeled bottles were sampled for DTAF bacteria counts. The four dissolved ${ }^{133} \mathrm{Ba}-$ spiked bottles were sampled at $t_{0}, t_{24}, t_{72}$, and $t_{120}$ for total and $0.2 \mu \mathrm{m}$ filterable ${ }^{133} \mathrm{Ba}$ as described previously. 
Table 5.3 - ${ }^{1}$ Size fractions and treatments set up for field experiment \#3 incubations

\begin{tabular}{ccc}
\multicolumn{2}{c}{${ }^{2}$ Radio-labeled incubations } & ${ }^{3}$ Non-labeled incubations \\
Series $1-$ bacteria + & Series 2 - ferrihydrite & \\
ferrihydrite & only & \\
$<200 \mu \mathrm{m}$ & $<200 \mu \mathrm{m}$ & $<200 \mu \mathrm{m}$ \\
$<20 \mu \mathrm{m}$ & $<20 \mu \mathrm{m}$ & $<20 \mu \mathrm{m}$ \\
$<200 \mu \mathrm{m}+$ & $<200 \mu \mathrm{m}+$ & $<200 \mu \mathrm{m}+$ \\
metabolic inhibitor & metabolic inhibitor & \\
$<1 \mu \mathrm{m}$ & $<1 \mu \mathrm{m}$ & \\
$<0.2 \mu \mathrm{m}$ & $<0.2 \mu \mathrm{m}$ &
\end{tabular}

1 - All types of incubations shown were set up in replicate.

2 - Radio-labeled incubations contained ${ }^{133} \mathrm{Ba} /{ }^{59} \mathrm{Fe}$ co-labeled colloidal ferrihydrite added at $1-2 \mu \mathrm{M}$ Fe concentration. Series 1 contained ${ }^{133} \mathrm{Ba} /{ }^{59} \mathrm{Fe}$ ferrihydrite added in association with heat-killed bacteria; Series 2 contained ${ }^{133} \mathrm{Ba} /{ }^{59} \mathrm{Fe}$ ferrihydrite only.

3 - Non-labeled incubations contained only DTAF-stained bacteria to follow grazing rates (see text). 
Field experiment \#3 - Results - Figure 5.7 shows the kinetics of removal of the DTAFstained bacteria from the non-labeled incubations in field experiment \#3. Results are fairly similar to what was observed in field experiment \#1. The metabolically inhibited sample had a greatly reduced rate of bacteria removal, and the non-inhibited $<200 \mu \mathrm{m}$ and $<20 \mu \mathrm{m}$ incubations demonstrated a removal rate of about $18 \%$ of the DTAF-stained bacteria per day. This is very similar to the removal rate of $20 \%$ per day observed in field experiment \#1, which is consistent with the similar concentrations of bacteria added at $t_{0}$ in both experiments.

Accompanying the removal of DTAF-stained bacteria in the actively grazing cultures was a dramatic wall loss of ${ }^{133} \mathrm{Ba}$ and ${ }^{59} \mathrm{Fe}$ activity. This is shown in Figure 5.8 for both series 1 radio-labeled incubations (A; Fe colloids added with bacteria) and series 2 (B; Fe colloids added alone). Wall loss was much greater in the non-inhibited $<200 \mu \mathrm{m}$ and $<20 \mu \mathrm{m}$ treatments in both series. The rate of wall loss in Figure 5.8, in fact, is very similar to the rate of removal of DTAF-stained bacteria in Figure 5.7 (both $17-18 \%$ per day). This indicates that wall loss is likely a function of the grazing process, as hypothesized previously. The loss of ${ }^{133} \mathrm{Ba}$ to bottle walls shown in Figure 5.8, as in previous experiments, is interpreted to be due to the adhesion of ${ }^{133} \mathrm{Ba}$-impregnated colloidal ferrihydrite to bottle walls. In the smaller $<200 \mu \mathrm{m}$ and $<20 \mu \mathrm{m}$ incubations spiked with dissolved ${ }^{133} \mathrm{Ba}$, no wall loss of dissolved ${ }^{133} \mathrm{Ba}$ was observed by day 5 of the incubation relative to the dissolved ${ }^{133} \mathrm{Ba}$ activity added to each bottle at $\mathrm{t}_{0}$. Uptake of ${ }^{133} \mathrm{Ba}$ onto particles $>0.2 \mu \mathrm{m}$ in those incubations by day 5 was $1.45 \pm 0.6 \%$ in the $<200 \mu \mathrm{m}$ incubations, and $0.07 \pm 0.02 \%$ in the $<20 \mu \mathrm{m}$ incubations. Thus dissolved ${ }^{133} \mathrm{Ba}$ was again shown to remain stable in the solution phase over a 5 day incubation. The greater uptake of ${ }^{133} \mathrm{Ba}$ onto particles in the $<200 \mu \mathrm{m}$ size fraction incubations could have been due to the formation of barite in aggregates in those bottles (Bishop, 1988; Dymond et. al., 1992). Microscopic examination of the natural assemblage in the seawater indicated that, like the seawater in field experiment \#2, the indigenous biological community was fairly productive, containing a number of phytoplankon, including chain- 


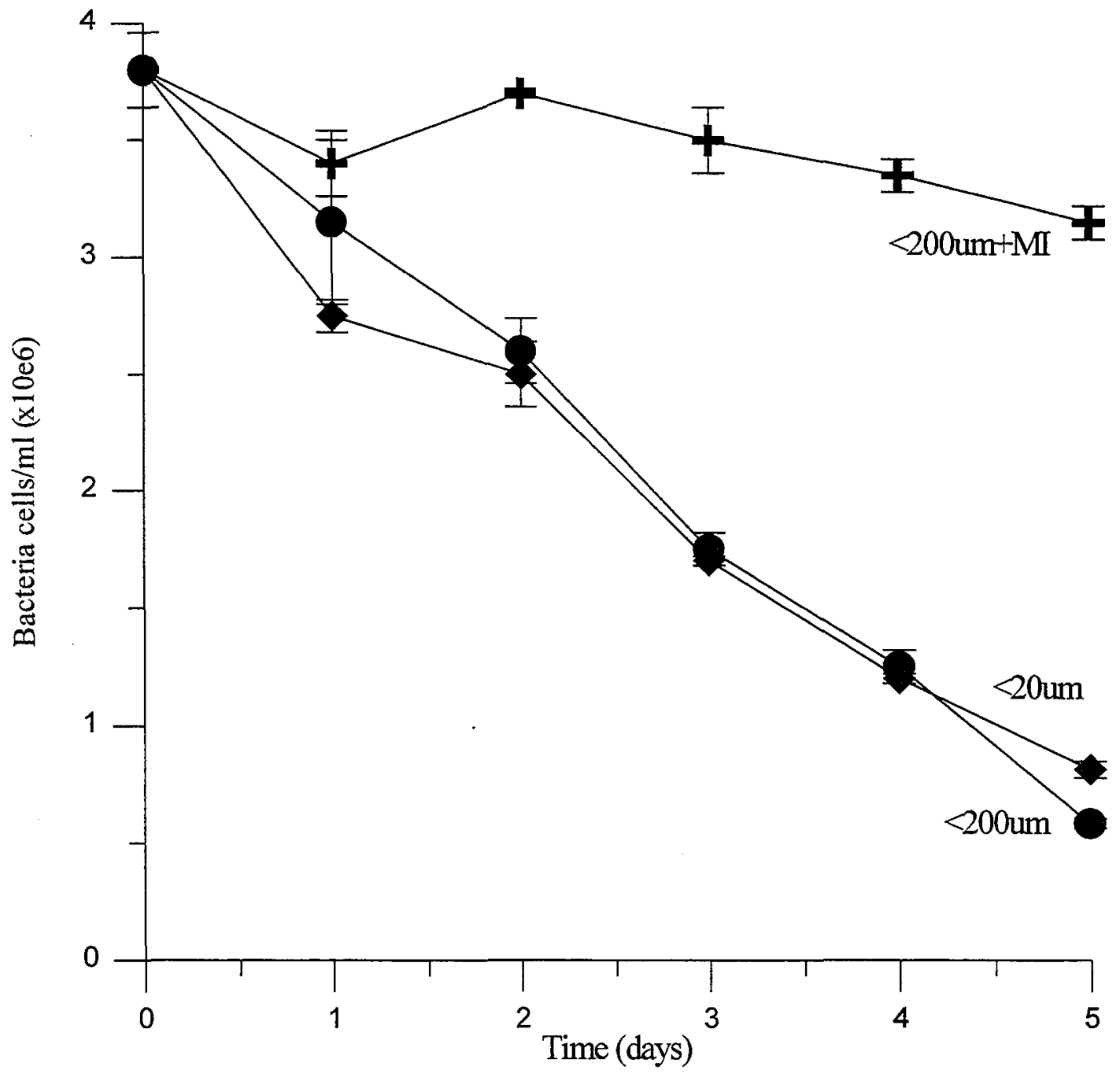

Figure 5.7 - Field experiment \#3 - Removal of DTAF-stained bacteria in different incubation treatments over time 
A
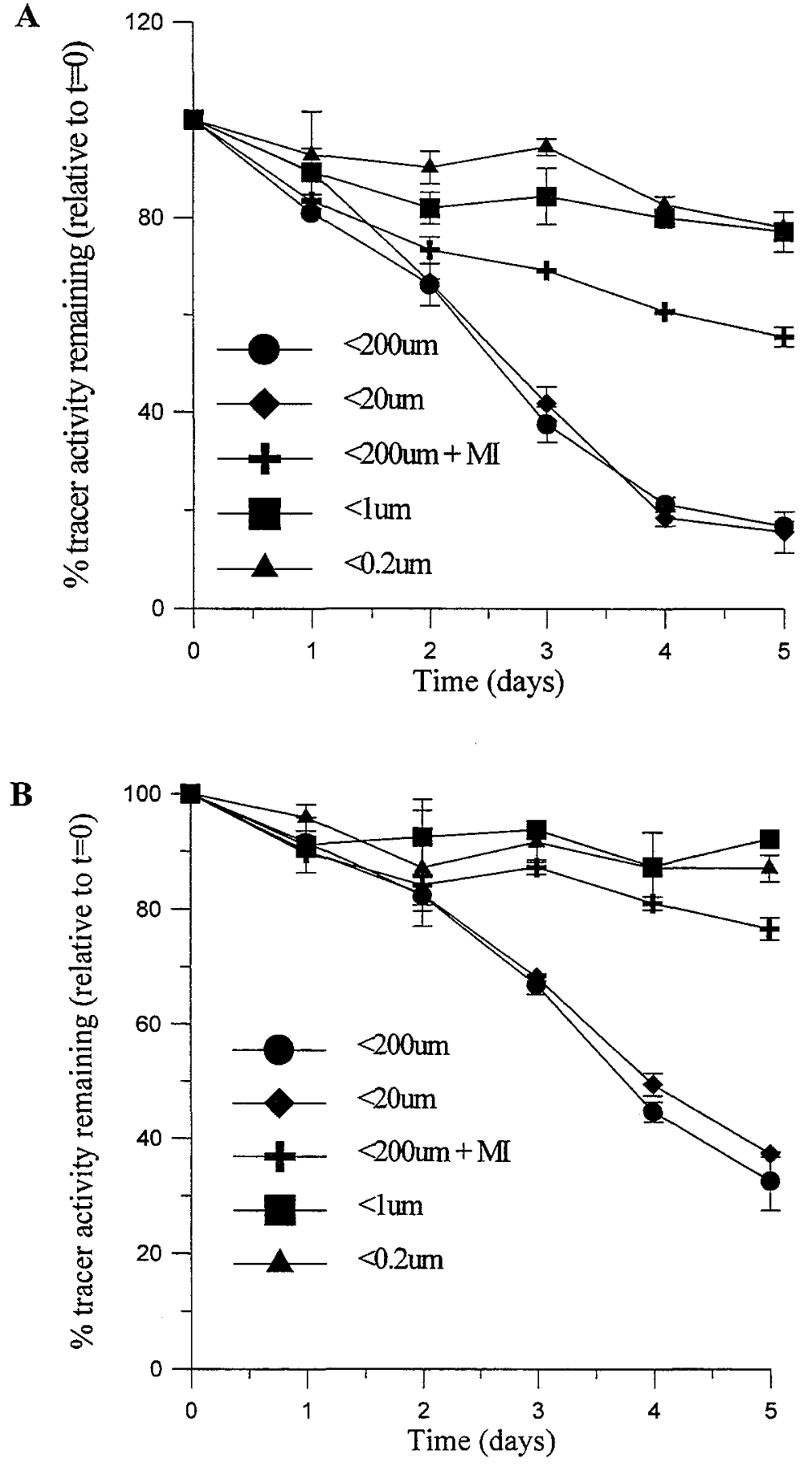

Figure 5.8 - Field experiment \#3 - loss of Ba-133 activity over time (expressed as $\%$ of Ba- 133 activity at $t=0$ ) in incubation treatments due to wall loss of $\mathrm{Ba}-133$ ferrihydrite

A. Series $1, \mathrm{Ba}-133$ ferrihydrite added with bacteria

B. Series 2, Ba-133 ferrihydrite added alone 
forming Chaetoceros-like species. The presence of diatoms in the $<200 \mu \mathrm{m}$ size class incubations could have led to some ${ }^{133} \mathrm{Ba}$ uptake due to barite formation.

Aside from its implications for the interpretation of dissolved ${ }^{133} \mathrm{Ba}$ data, the wall loss shown in Figure 5.8 is an interesting phenomenon in its own right. Throughout this series of field experiments and also in the laboratory experiments described previously, wall loss of ${ }^{133} \mathrm{Ba}$-impregnated ferrihydrite has been associated with the activity of protozoan grazers. In field experiments in particular, very efficient removal of colloidal ferrihydrite from solution has been observed to occur coincident with grazing activity. This indicates that protozoan grazers may produce highly surface-active material which associates with colloidal ferrihydrite and facilitates its adsorption to bottle walls. The production of surface active material by grazers is consistent with previous observations in culture experiments in Chapters 2 and 3 - surface films seen in grazing cultures; difficulty using CSV techniques because of surfactants, etc. The observation of copious surfactant production by grazers has significant implications for the role of heterotrophic protists in the cycling of organic carbon in marine environments. Grazer-produced surfactants may play an important role in the aggregation of particulate and colloidal organic material and modification of aquatic surface chemistry.

As in previous experiments, the effect of wall loss on interpretation of dissolved ${ }^{133} \mathrm{Ba}$ data must be considered. Percent dissolved ${ }^{133} \mathrm{Ba}$ produced in series 1 and series 2 incubations is shown in Figure 5.9 normalized to ${ }^{133} \mathrm{Ba}$ activity at $\mathrm{t}_{0}$. In Figure 5.10, the same data is shown normalized to the total ${ }^{133} \mathrm{Ba}$ activity in each incubation treatment at each time point. While $\%$ dissolved ${ }^{133} \mathrm{Ba}$ is perceptibly higher in the actively grazing treatments in both cases, the effect is much more pronounced in Figure 5.10, with 50\% and $25 \%$ solubilization of the total ${ }^{133} \mathrm{Ba}$ present in the grazing cultures in series 1 (Fig. $5.10 \mathrm{~A}$ ) and series 2 (Fig. $5.10 \mathrm{~B}$ ), respectively, vs. $<5 \%$ solubilization in the non-grazing controls for both series.

The data in Figure 5.9, \% dissolved ${ }^{133} \mathrm{Ba}$ normalized to $\mathrm{t}_{0}$, can be taken as a minimum estimate of the effects of the natural grazing consortium on colloidal ferihydrite reactivity. The rate of dissolved ${ }^{133} \mathrm{Ba}$ increase for all treatments is the same from $\mathrm{t}_{0}$ to 

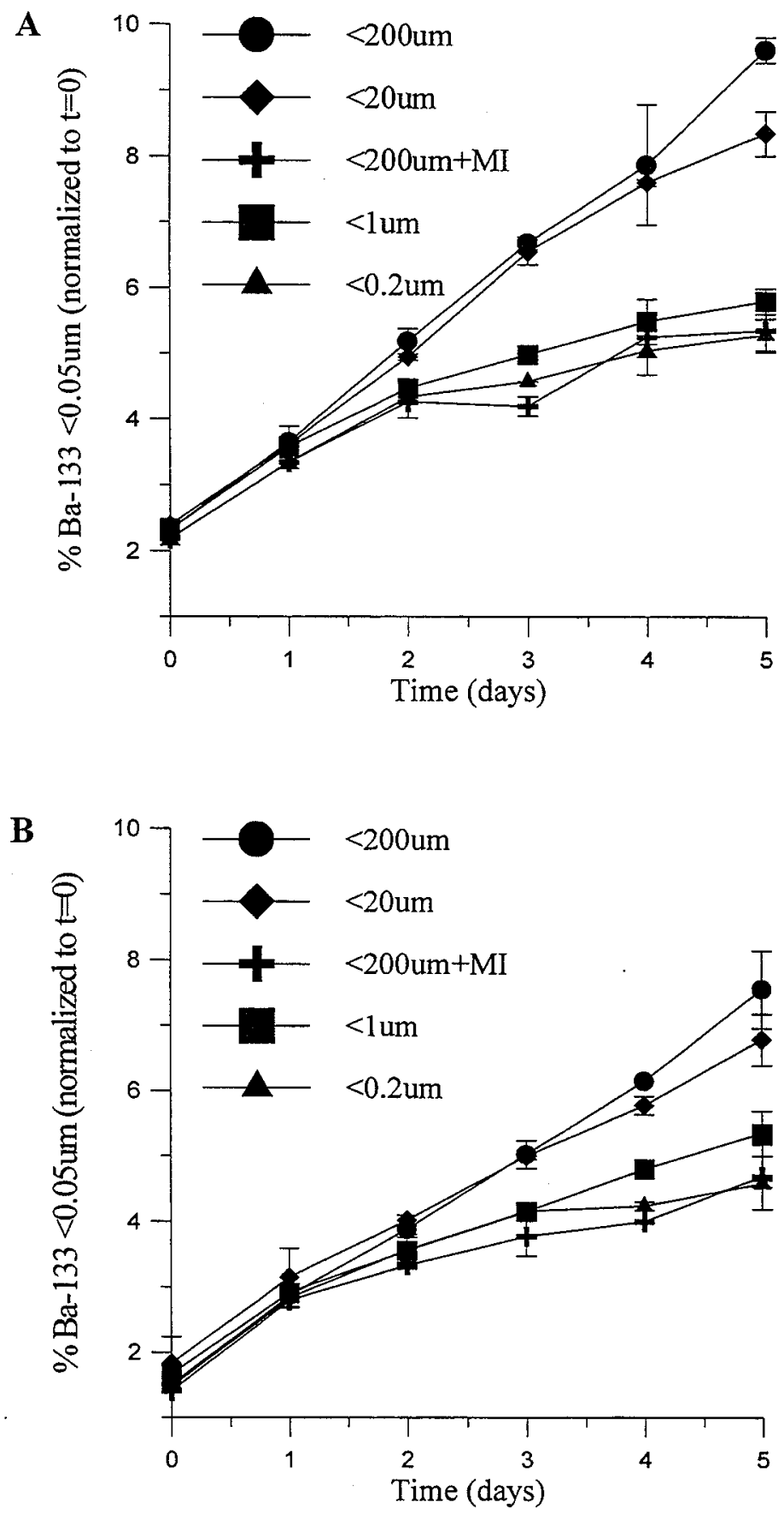

Figure 5.9 - Field experiment $\# 3$ - \% dissolved Ba-133 production in different incubation treatments over time, normalized to $t=0$ A. Series 1, Ba-133 ferrihydrite added with bacteria B. Series 2, Ba-133 ferrihydrite added alone 

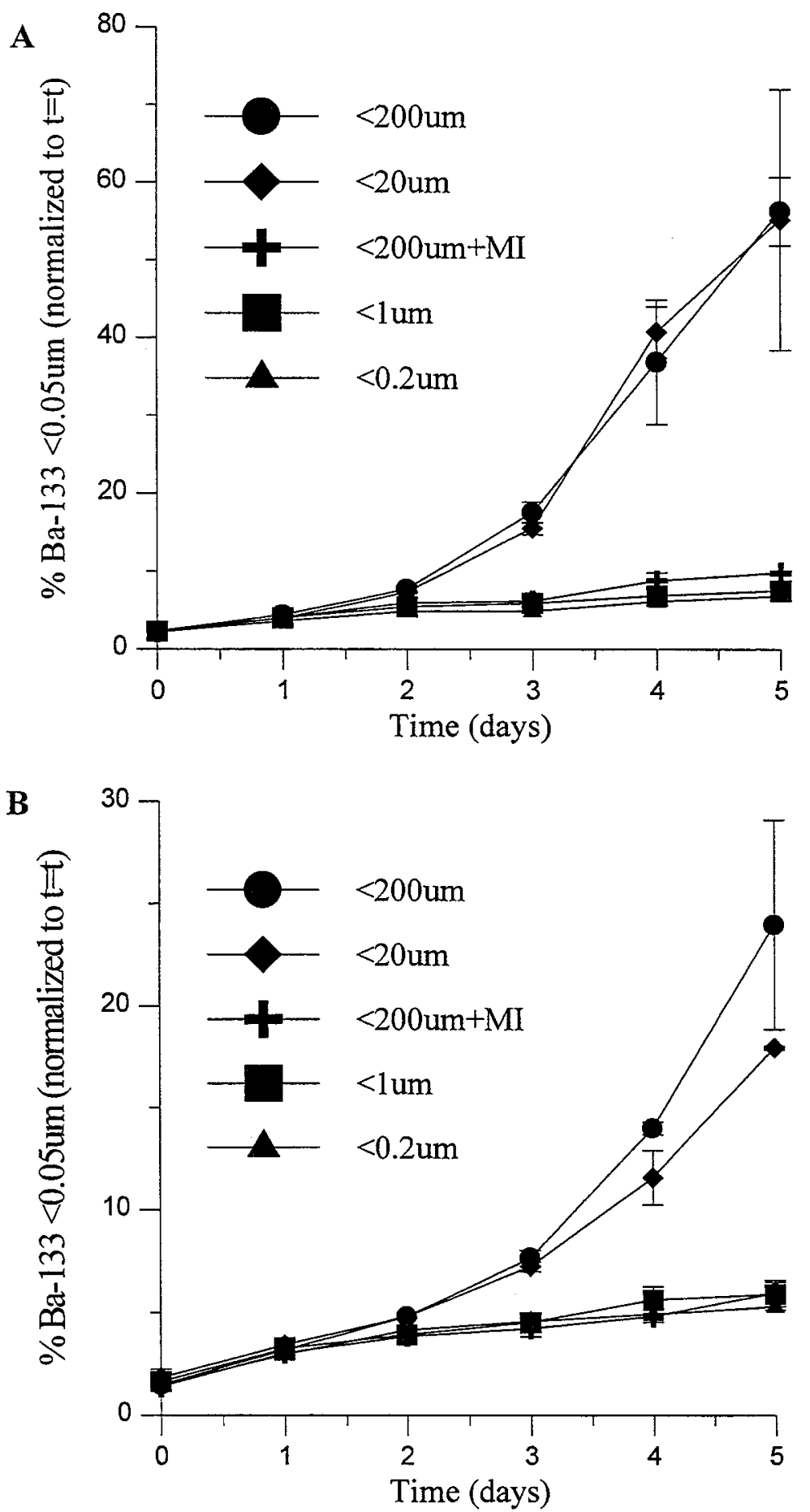

Figure 5.10 - Field experiment \#3 - \% dissolved $\mathrm{Ba}-133$ production in different incubation treatments over time, normalized to $t=t$ A. Series 1, Ba-133 ferrihydrite added with bacteria B. Series 2, Ba-133 ferrihydrite added alone 
$t_{24}$. This rate is largely a function of the initial release of ${ }^{133} \mathrm{Ba}$ from the ${ }^{133} \mathrm{Ba}-$ impregnated colloids upon addition to seawater, due to either a small excess of ${ }^{133} \mathrm{Ba}$ that was not rinsed away by dialysis, ion exchange with alkaline earths in seawater, and/or conformational changes in the ferrihydrite that occur due to the change from a Milli-Q water matrix to seawater. In the non-grazing controls, particularly in the metabolically inhibited and $<0.2 \mu \mathrm{m}$ filtered controls, this rate flattens out perceptibly after about 48 hours. The slow rate of increase in dissolved ${ }^{133} \mathrm{Ba}$ in the controls after 48 hours is most likely due to thermal dissolution and/or maturation reactions that cause a slow release of ${ }^{133} \mathrm{Ba}$ from the $\mathrm{Fe}$ oxide matrix. In the grazing incubations, the relatively fast initial rate of increase in dissolved ${ }^{133} \mathrm{Ba}$ is maintained over the entire 5 day period, presumably due to the dissolution of colloidal ferrihydrite by the grazers.

The rate of increase in dissolved ${ }^{133} \mathrm{Ba}$ in Figure 5.9A, for the non-inhibited $<200 \mu \mathrm{m}$ incubations in radio-labeled series 1 , is about $1.5 \%$ per day over the entire time course. In the non-grazing controls, the rate of increase in dissolved ${ }^{133} \mathrm{Ba}$ after 48 hours (the period of equilibration and relatively faster ${ }^{133} \mathrm{Ba}$ release) is about $0.3 \%$ per day. Subtracting this from the rate of increase in the grazing cultures, this gives a rate of increase in dissolved ${ }^{133} \mathrm{Ba}$ due to grazing of about $1.2 \%$ per day. Relative to the consumption of DTAFlabeled bacteria in those cultures (Fig. 5.7A, $-17.6 \%$ per day), this indicates a colloidal ferrihydrite dissolution efficiency of the indigenous grazer population of about 7\%. This figure is in fairly good agreement with the estimated dissolution efficiency for a natural grazing consortium derived previously from the data in Table 5.1 (8\%). The measured rate of colloidal iron oxide dissolution in this field experiment, $1.2 \%$ per day, is also in relatively good agreement with the lower end of the range of the first order rate constant for protozoan-mediated dissolution of colloidal iron oxides in natural waters derived earlier in this chapter, $1.7 \%$ per day $\left(=0.7 \times 10^{-3} \mathrm{~h}^{-1}\right)$. This value corresponds to a turnover rate of colloidal iron oxides by grazers of about $20 \%$ per day, as observed in this experiment.

The results for the series 2 incubations were actually fairly similar to series 1 . The rate of increase in dissolved ${ }^{133} \mathrm{Ba}$ in the non-inhibited $<200 \mu \mathrm{m}$ incubation in Figure 5.9B, 
corrected in the same way as above for dissolution after 48 hours in the controls, is about $1 \%$ per day. The apparent difference between the grazing cultures and controls in series 2 (Figure 5.9B) is lessened due to the somewhat elevated rate of dissolution in the $>1 \mu \mathrm{m}$ control in series 2. As in field experiment \#1, the observed dissolution in the $>1 \mu \mathrm{m}$ control could be due to prokaryote activity, or due to the presence of small flagellates not removed by $1 \mu \mathrm{m}$ filtration. One possible factor contributing to the difference between series 1 and series 2 is that the enrichment of series 1 systems with $3.8 \times 10^{6}$ cells $/ \mathrm{ml}$ bacteria likely caused an increase in the grazer population by day 5 . This could account for the somewhat elevated rate of colloidal ferrihydrite dissolution in series 1 relative to series 2.

Another contributing factor could be lower ingestion rates of the colloidal $\mathrm{Fe}$ in series 2. Since the ferrihydrite was added by itself without being pre-associated with bacterial cells as in series 1 , it could have been scavenged by a variety of indigenous particles present in the seawater, many of which may not have been amenable to grazing. Still, the decrease in the total ${ }^{133} \mathrm{Ba}$ activity in the non-inhibited $<200 \mu \mathrm{m}$ and $<20 \mu \mathrm{m}$ incubations in series 2 (Figure 5.8B) indicates that the colloidal iron was ingested by grazers relatively efficiently, at least during the latter part of the experiment. If wall loss is directly related to the grazing process, then the data in Figure 5.8B indicate that the turnover of iron oxides in the series 2 radio-labeled incubations was close to $17 \%$ per day between day 1 and day 5 . This may reflect an average value for the turnover of naturallyoccuring iron oxides by grazers in surface waters. This value is dependent, however, on the overall rate of clearance of protozoan grazers in the system.

\section{CONCLUSION}

In Chapter 4 of this thesis a novel tool, ${ }^{133} \mathrm{Ba}$-impregnated ferrihydrite, was presented for the study of reactions of iron oxides in seawater. In this chapter, this tool was applied to the study of protozoan grazer-mediated transformations of colloidal iron oxides. In 
both laboratory and field studies, results with the ${ }^{133} \mathrm{Ba}$ method converge on a value of 7$8 \%$ for the efficiency of protozoan-mediated dissolution of colloidal ferrihydrite in natural waters. This means that protozoan grazing consortia dissolve about $7-8 \%$ of the iron oxides which they ingest. Translating this efficiency into an overall rate constant for protozoan-mediated dissolution of iron oxides in surface waters requires some knowledge of the turnover rate of iron oxides by protists in the system. Results indicate that, for iron oxides added to the system and allowed to associate with the naturally-occurring particle pool (series 2 in field experiment \#3), this turnover rate is about $20 \%$ per day in coastal systems. This is admittedly a rough estimate. At a turnover rate of about $20 \%$ per day, field measurements in this chapter (Fig. 5.9) indicate that protozoan grazers may be expected to dissolve iron oxides in surface waters at a rate of $1-2 \%$ per day. This is a minimum estimate, since the removal of grazer-available colloidal ferrihydrite due to wall loss has not been taken into account.

This rate estimate is of course operationally defined by the model phase used here as representative of commonly-occurring refractory iron phases in natural waters: 6-line ferrihydrite, the most ordered form of this sub-crystalline phase (Schwertmann and Cornell, 1991). Other workers have used similar material as a model phase for refractory iron solids (Wells et. al., 1991a; Johnson et. al., 1994), and there is cause to believe that this model phase reasonably approximates, at least in terms of its chemical reactivity, the vast and ill-defined array of inorganic and mixed inorganic/organic forms of iron oxide that occur in natural systems (Cornell and Schwertmann, 1996; Wells and Mayer, 1991b; Pizarro et. al., 1995).

A truer sense of the significance of the work in this chapter may be obtained by comparing protozoan-mediated dissolution of iron oxides to other processes known to be important in the redox cycling of iron in oxic surface waters. Chief among these processes is photochemistry (Sulzberger and Laubscher, 1995; Johnson et. al., 1994). At the conclusion of Chapter 3, a very rough estimate was made of the rate of protozoanmediated dissolution of iron oxides in natural waters, based on average values for protozoan concentrations and clearance rates in seawater (Berninger et. al., 1991). Based 
on this estimate, protozoan-mediated dissolution of iron oxides appeared to have a firstorder rate constant about ten times less than one published value for the maximal rate of photochemically-mediated dissolution of iron oxides (Wells and Mayer, 1991a). The argument was made that, if the effect of light attentuation with depth, diurnal cycling, and cloud cover on the photoreduction rate is taken into account, then spatially- and temporally-averaged rates of photochemical and protozoan-mediated dissolution of refractory iron phases in surface waters may be of similar magnitude.

It is difficult to quantitatively compare the rate of protozoan-mediated dissolution with the rate of photoreductive dissolution of iron oxides. A wide range exists in published values for the rate of iron oxide photoreduction in seawater at $\mathrm{pH} 8$. For example, the rate constant obtained by Wells and Mayer (1991a) which has been used here as a basis for comparison $\left(1.8 \times 10^{-2} \mathrm{~h}^{-1}\right)$ is at least a factor of 10 lower than photoreduction rate constants derived by Johnson et. al., (1994). Several factors could account for this difference, including some questionable assumptions made by Johnson et. al., (1994) as to the $\mathrm{pH}$ and wavelength dependence of iron oxide photoreduction. Few measurements have been made of iron oxide photoreduction rates in seawater, and there are significant differences amongst these various investigators (eg. Wells and Mayer, 1991a; Johnson et. al., 1994; Waite and Morel, 1984b) in the concentration of iron oxide, the degree of reactivity of the iron oxide phase, experimental conditions, and detection methods. Thus there is not a universally-accepted value for the rate of photochemically-mediated dissolution of iron oxides in seawater to which protozoan-mediated dissolution can be compared.

Another difficulty that arises in this comparison is that the few photoreduction rates that have been measured represent near-surface maximum rates, which must be extrapolated and integrated to obtain average values with depth and over time. Johnson et. al., (1994) attempted to do this with a model of iron cycling in seawater at the equatorial Pacific. Their maximum photoreduction rate in the upper $10 \mathrm{~m}, 25 \mathrm{pmol} \mathrm{Fe} \mathrm{l}^{-1}$ $\mathrm{h}^{-1}$, dropped to an average rate of only $2 \mathrm{pmol} \mathrm{Fe}^{-1} \mathrm{~h}^{-1}$ when integrated over a 24 hour cycle at light intensities from the surface to the $0.1 \%$ light level $(75 \mathrm{~m})$. This model was 
for an oligotrophic open ocean region. As shown by Wells et. al., (1991b), the attenuation of photochemically active light occurs at much shallower depths in coastal areas (5-10 m) vs. open ocean waters $(100 \mathrm{~m})$. The depth of light penetration is also dependent on the assumed wavelength dependence of iron oxide photoreduction, which is currently not very well-constrained.

Bearing in mind the inherent difficulties in the comparison as described above, it may nonetheless be useful to compare photochemically-mediated dissolution of colloidal iron oxides with protozoan-mediated dissolution, both as determined by the ${ }^{133} \mathrm{Ba}$ technique. This comparison at least involves the same iron oxide phase, with reaction rates determined by the same method. In Chapter 4, the rate of colloidal iron oxide dissolution by photolysis was measured in natural sunlight in filtered and unfiltered Vineyard Sound seawater, at $1 \mu \mathrm{M}$ concentration Fe. In unfiltered seawater, the maximal initial photolysis rate was determined to be about $12 \%$ per day. In this chapter, the rate of protozoanmediated dissolution of colloidal iron oxides has been measured in a variety of laboratory culture experiments and in field incubation experiments with natural consortia. As described above, these measurements converge on a value of $1-2 \%$ per day for protozoanmediated dissolution of colloidal iron oxides in natural waters.

Thus, in a rate comparison on the same iron oxide phase, at the same concentration in Vineyard Sound seawater, as determined by the same ${ }^{133} \mathrm{Ba}$ measurement technique, it is estimated that protozoan-mediated dissolution of colloidal iron oxides in natural waters proceeds at a rate which is about one-tenth of the maximal, noon-time near-surface rate of photochemically-mediated dissolution of iron oxides. The relative magnitude of these two rates is similar to that obtained in the previous comparison at the end of Chapter 3. Again, it is argued on the basis of these measurements that, on a time- and depthintegrated basis, protozoan grazers may be equal in importance to photochemistry as an influence on the redox cycling of refractory iron solids in oxygenated seawater.

As shown in the experiments in this chapter, turnover rate is a key parameter in determining the rate of protozoan-mediated dissolution of refractory iron solids. Turnover rate will vary depending on the overall clearance rate of protists in the system, 
and depending on the degree to which iron solids are associated with "grazable" particles. Before this issue can be addressed, more information is needed on the physico-chemical speciation of iron solids and colloids in seawater. In the experimental systems used in this chapter, turnover rates were reduced due to wall loss of iron oxides. In a natural system, surface-active substances would be more likely to remain associated with suspended particles and probably be cycled through the grazing process several times, resulting in higher dissolution rates than what has been observed in these laboratory model systems. Further work is needed in a variety of environments in order to quantify the relationship between the turnover of bacterial biomass by protozoan grazers and the cycling of refractory iron solids by phagotrophic ingestion. 


\section{References}

- Berninger, U.-G., D.A. Caron, R.W. Sanders and B.J. Finlay. 1991. Heterotrophic flagellates of planktonic communities: their characteristics and methods of study. p. 3956. In The Biology of Free-living Heterotrophic Flagellates, Systematics Association Special Volume 45. Patterson, D.J. and J. Larsen (eds.). Clarendon, Oxford.

- Bishop, J.K.B. 1988. The barite-opal-organic carbon association in oceanic particulate matter. Nature. 332: 341-343.

- Capriulo, G.M. 1990. Feeding-related ecology of marine protozoa. p. 186-259. In Ecology of Marine Protozoa. Capriulo, G.M. (ed.). Oxford University Press, NY. - Caron, D., E.L. Lim, G. Miceli, J.B. Waterbury and F.W. Valois. 1991. Grazing and utilization of chroococcoid cyanobacteria and heterotrophic bacteria by protozoa in laboratory cultures and a coastal plankton community. Mar. Ecol. Prog. Ser. 76: 205217.

- Cornell, R.M. and U. Schwertmann. 1996. The Iron Oxides: Structure, Properties, Reactions, Occurrence and Uses. VCH, New York, NY.

- Dymond, J., E. Suess and M. Lyle. 1992. Barium in deep-sea sediment: A geochemical proxy for paleoproductivity. Paleoceanography 7: 163-181.

- Johnson, K.S., K.H. Coale, V.A. Elrod and N.W. Tindale. 1994. Iron photochemistry in the equatorial Pacific. Mar. Chem. 46: 319-334.

- Pizarro, J., N. Belzile, M. Filella, G.G. Leppard, J.-C. Negre, D. Perret and J. Buffle. 1995. Coagulation/sedimentation of submicron iron particles in a eutrophic lake. Wat. Res. 29: 617-632.

- Rich, H.W. and F.M.M. Morel. 1990. Availability of well-defined iron colloids to the marine diatom Thalassiosira weissflogii. Limnol. Oceanogr. 35: 652-662.

- Schwertmann, U. and R.M. Cornell. 1991. Iron Oxides in the Laboratory. VCH, New York, NY. 
- Sherr, B.F., E.B. Sherr and R.D. Fallon. 1987. Use of monodispersed, fluorescently labeled bacteria to estimate in situ protozoan bacterivory. Appl. Env. Micobiol. 53: 958965.

- Sherr, B.F., E.B. Sherr, T.L. Andrew, R.D. Fallon and S.Y. Newell. 1986. Trophic interactions between heterotrophic protozoa and bacterioplankton in estuarine water analyzed with selective metabolic inhibitors. Mar. Ecol. Prog. Ser. 32: 169-179.

- Sulzberger, B. and H. Laubscher. 1995. Reactivity of various types of iron(III) (hydr)oxides towards light-induced dissolution. Mar. Chem. 50: 103-115.

- Valiela, I. 1995. Marine Ecological Processes. Springer, New York.

- Waite, T.D. and F.M.M. Morel. 1984a. Photoreductive dissolution of colloidal iron oxide: Effect of citrate. J. Colloid. Interface Sci. 102:121-137.

- Waite, T.D. and F.M.M. Morel. 1984b. Photoreductive dissolution of colloidal iron oxides in natural waters. Environ. Sci. Technol. 18: 860-868.

- Wells, M.L. and L.M. Mayer. 1991a. The photoconversion of colloidal iron hydroxides in seawater. Deep-Sea Res. 38: 1379-1395.

- Wells, M.L. and L.M. Mayer. 1991b. Variations in the chemical lability of iron in estuarine, coastal and shelf waters and its implications for phytoplankton. Mar. Chem. 32: $195-210$.

- Wells, M.L., L.M. Mayer and Guillard, R.R.L. 1991a. A chemical method for estimating the availability of iron to phytoplankton in seawater. Mar. Chem. 33: 23-40.

- Wells, M.L., L.M. Mayer, O.F.X. Donard, M.M. de Souza Sierra and S. Ackleson. 1991b. The photolysis of colloidal iron in the oceans. Nature 353: 248-250.

- Wu, J. and G.W. Luther. 1995. Complexation of Fe(III) by natural organic ligands in the Northwest Atlantic Ocean by a competitive ligand equilibration method and kinetic approach. Mar. Chem. 50: 159-177. 


\section{CHAPTER 6}

\section{Synthesis and Conclusions}

In the oceans, the biological cycling of carbon is intimately tied to and interactively influenced by the geochemical cycling of trace metals (Bruland et. al., 1991). Additional layers of complexity in this relationship are continually being revealed by ongoing studies in biological and chemical oceanography. Much research on trace metal-biota interactions in the marine environment is currently focused on the speciation and bioavailability of dissolved trace metals. To the extent that biological interaction with trace metals on particles is considered, planktonic organisms are largely viewed either in terms of their potential to convert dissolved trace metals into particulate form via active uptake or passive scavenging; or in terms of their ability to repackage particulate trace metals into biomass or rapidly sinking fecal pellets.

Heterotrophic protists are a class of planktonic organisms whose importance in upper ocean nutrient cycles has been recognized only in the past two decades (Azam et. al., 1983). These grazers may play a unique and important role in the cycling of particleassociated trace metals in marine systems. As the primary sink for bacteria and similarlysized algal cells, heterotrophic protists encounter and ingest a major pool of surfaceassociated trace metals. Once phagocytized, ingested trace metals are subjected to the reactive conditions which accompany intracellular digestion. The large chemical shift from bulk seawater to the grazer food vacuole can cause changes in metal redox state and coordination chemistry that affect trace metal fate and transport in marine systems.

The hypothesis that protozoan grazers can significantly influence the geochemical cycling of trace metals in the oceans has provided the impetus for this thesis. Focusing on strongly hydrolyzed, particle reactive metals as species most likely to undergo nonequilibrium reactions due to grazer ingestion, this research has attempted to evaluate the potential for protozoan grazers to transform trace metals associated with various solid phases. Throughout this work, model systems have been employed as a tool to identify fundamental processes and the mechanisms which drive them. The conclusions drawn 
from these studies should serve as a basis for further research on trace metal-biota interactions as mediated by phagotrophy.

\section{Specific conclusions/contributions of this thesis}

In Chapter 2, a series of experiments was conducted to examine the fate of trace metals ( $\mathrm{Fe}, \mathrm{Th}, \mathrm{Cr}$ ) associated with bacteria cells, upon ingestion of bacteria by protozoan grazers. These metals were chosen for study based on their particle-reactive chemistry and biological/geochemical importance. Several different protozoan species were characterized with respect to their effects on trace metal partitioning, and the cycling of organic carbon in grazing cultures relative to trace metals was also studied. While these experiments were not totally conclusive, they did generate a number of interesting, original, and potentially significant observations.

One finding was that protozoans are apparently equally able to mobilize intracellular and extracellularly-bound trace metals. This is consistent with what is known about the chemistry of phagotrophic digestion, and is also in marked contrast to what has been found in studies of larger zooplankton grazers, which practice extracellular digestion (Reinfelder and Fisher, 1991). Protozoans can therefore be expected to solubilize trace metals (such as Th) bound non-specifically to cell surfaces. In some experimental systems, trace metals were regenerated into the dissolved phase by the grazing activity of protists and remained stable in dissolved form, despite the presence of abundant particle surface area in cultures for trace metal adsorption. Ultrafiltration results indicate that, of those trace metals in the $<0.2 \mu \mathrm{m}$ size class, a major fraction was filterable through a 10,000 molecular weight cut-off. For particle-reactive trace metals such as Fe and Th to remain stable as truly dissolved species in such particle-rich model systems, this implies that the trace metals were regenerated by the protozoan in the form of stable metalorganic complexes. This indicates that protozoan grazers may act to increase the residence time and (potentially) affect the bioavailability of trace metals in the upper oceans by facilitating the formation of metal-organic complexes. 
In some experimental systems, protozoan grazing appeared to lead to the formation of colloidal material. Trace metal scavenging by colloids and aggregation were hypothesized to lead to an increase in the metal/carbon ratio of larger, aggregated particles, via a type of "colloidal pumping" mechanism (Honeyman and Santschi, 1989). The degree to which colloidal trace metal species were formed and to which aggregation occurred was influenced by several factors, including prey surface chemistry and specifics of protozoan metabolism. Further work is needed to characterize the relative importance of these contributing factors, and to assess the relevance of these results for less concentrated, natural marine systems.

In Chapter 3, the ability of protozoan grazers to cause dissolution of iron oxide mineral phases was investigated. Iron oxides are ubiquitous components of aquatic systems, and play an important role in the transport and chemical cycling of organic compounds (Cornell and Schwertmann, 1996). Few dissolution pathways are known for iron oxides in oxygenated waters, aside from photochemistry and ligand-mediated dissolution. Four independent techniques were employed in Chapter 3 in order to demonstrate in laboratory grazing cultures that dissolution of iron oxide phases can take place within the microenvironment of the grazer food vacuole. This was the first demonstration of a novel iron oxide reaction mechanism in aqueous systems, mediated by the digestive chemistry of phagotrophic organisms. Ingestion of colloidal ferrihydrite by protozoan grazers was shown to cause an increase in both the chemical reactivity and bioavailability of iron oxides. This finding has implications which extend to both the redox cycling of iron in natural waters and the biological utilization of iron solids.

In order to expand upon the laboratory studies of protozoan-mediated dissolution of iron oxides in Chapter 3, a novel method of studying the reactions of iron oxide phases in seawater was developed and presented in Chapter 4. This technique, the "inert tracer method" is based upon the synthesis of colloidal ferrihydrite uniformly impregnated with the gamma-emitting radiotracer ${ }^{133} \mathrm{Ba}$. When ${ }^{133} \mathrm{Ba}$-impregnated colloidal ferrihydrite is added to seawater, disruption/dissolution of the iron oxide matrix leads to the release of ${ }^{133} \mathrm{Ba}$ into the dissolved phase. Because $\mathrm{Ba}$ is non-particle reactive and is not biologically 
utilized, it remains stably dissolved. The accumulation of ${ }^{133} \mathrm{Ba}$ in the dissolved phase provides a relative measure of the rate and extent of reaction undergone by the iron oxide phase. This method is particularly well-suited to the measurement of very slow iron oxide reactions. In addition, because ${ }^{133} \mathrm{~B}$ a release and detection is independent of the post-reaction fate of the iron itself, the inert tracer method provides a measure of the reaction rate of iron oxides regardless of the removal of reactive iron in the system, for example by organic complexation or biological uptake.

In Chapter 4 , the ${ }^{133} \mathrm{Ba}$-impregnated ferrihydrite was tested in a variety of codissolution experiments in order to demonstrate that $\mathrm{Fe}$ and ${ }^{133} \mathrm{Ba}$ dissolve congruently upon reaction of the iron oxide phase. This indicates that ${ }^{133} \mathrm{Ba}$ is incorporated uniformly thoughout the oxide matrix as a trace component. The inert tracer method was applied in a variety photochemical irradiation experiments with iron oxides in seawater, and results were generally consistent with previous studies of iron oxide photolysis in natural waters (Waite and Morel 1984a and b; Wells and Mayer 1991). Additional ligand/thermal dissolution studies in Chapter 4 demonstrated the utility of the inert tracer method for quantifying slow reaction rates. This method is a truly original means of studying the reaction of iron oxides under ambient conditions in seawater, and is unsurpassed in its ability to detect iron oxide reactions with low specific rate constants. It may prove useful in future studies of the reaction of iron oxides in seawater within biological microenvironments, or via ligand-mediated dissolution.

In Chapter 5, the inert tracer method was applied in a series of laboratory experiments and field incubation studies in order to determine quantitatively the rate of protozoanmediated dissolution of colloidal ferrihydrite. In laboratory culture experiments with the microflagellate grazer Cafeteria, the rate of ${ }^{133} \mathrm{Ba}$-ferrihydrite dissolution by an exponential-phase culture of this organism $\left(0.01-0.02 \mathrm{~h}^{-1}\right)$ was found to be close to the average dissolution rate of ${ }^{133} \mathrm{Ba}$-ferrihydrite in Vineyard Sound seawater measured in Chapter 4 over one hour of irradiation with a full spectrum $1000 \mathrm{~W} \mathrm{Hg} / \mathrm{Xe}$ lamp (0.02$0.03 \mathrm{~h}^{-1}$ ). Comparison of the ability of different protozoan species to dissolve colloidal ferrihydrite in laboratory culture using the inert tracer method revealed significant 
interspecies differences in the consumption-normalized dissolution efficiency of various protists.

By averaging together the dissolution efficiencies measured for different protozoan species, an estimated dissolution efficiency of about $8 \%$ was derived for a natural grazing consortium. This was combined with a range of published values for the turnover of bacterial biomass by grazers in marine systems to derive a value for the specific rate of protozoan-mediated dissolution of iron oxides in surface waters ranging from 1.7-5.8\% per day, depending on turnover rates. This laboratory-based estimate was supported by subsequent data from field incubation experiments using the inert tracer method with natural grazing consortia. In incubations of ${ }^{133} \mathrm{Ba}$-impregnated colloidal ferrihydrite with a natural grazing consortium, at a turnover rate of close to $20 \%$ per day, grazer-mediated dissolution of colloidal ferrihydrite was measured at a rate of about $1-2 \%$ per day.

The above rate for protozoan-mediated dissolution of colloidal iron oxides in natural waters is about ten times less than the maximal (noon-time, near surface) rate of ferrihydrite dissolution measured under natural sunlight irradiation in Vineyard Sound seawater using the inert tracer method in Chapter 4 (12\% per day). This comparison of photochemical vs protozoan-mediated dissolution of colloidal ferrihydrite, while still preliminary, suggests that protozoans could be a significant influence on the redox cycling of iron oxides in oxygenated surface waters. Light attenuation with depth and changes in light intensity with the time of day or weather conditions will, on a time and depth-integrated basis, tend to equalize the rate of photochemical and protozoanmediated dissolution of iron oxides in natural waters.

\section{Wider implications of this research}

Throughout this thesis, most recently at the beginning of this chapter, references have been made to the "interactive influences" between biota and trace metals in the marine environment. Traditionally, such influences have been viewed in terms of biota interacting with trace metals in the dissolved phase: the speciation of dissolved trace metals determines metal availability and toxicity to biota; and biota in turn are believed to 
be an important source of the organic ligands that determine the speciation of trace metals in the dissolved phase. This thesis research suggests a new framework within which to view the interaction of trace metals and biota, a framework which encompasses the idea of interactive influences between biota and solid phase trace metals, as well as dissolved trace metals. For organisms capable of phagotrophy, of which there are many in the marine environment, this new framework is more complete.

\section{Phagotrophy as a vector for trace metal-biota interaction}

Influence of phagotrophy on trace metal cycling - For the most part, this research has emphasized the active role of phagotrophic organisms (as epitomized by heterotrophic protists) in influencing the fate and mobility of trace metals in the marine environment. In general, phagotrophic processing of particle-associated trace metals should lead to the remobilization and enhanced residence time of trace metals in the water column. The finding that protists can regenerate trace metals associated with prey cells into the dissolved phase is not especially surprising - such processes have been suggested before (eg. Coale and Bruland, 1987), and other researchers have demonstrated protozoanmediated regeneration of trace metals from prey in previous studies (Hutchins et. al., 1993; Twiss and Campbell, 1995).

The more interesting implications of this research have to do with the form in which trace metals are regenerated from prey cells by protozoan grazers. In Chapter 2 , as mentioned previously, data indicate that $\mathrm{Fe}$ and $\mathrm{Th}$ may be regenerated from prey cells by protozoan grazers in the form of organic complexes. Trace metals were also found as colloidal species in some grazing experiments in Chapter 2. This implies that phagotrophic regeneration of trace metals is more complex than a simple conversion from solid phase to dissolved form. Recycling of trace metals via protozoan grazing may have an impact on the dissolved speciation of trace metals, thereby affecting trace metal bioavailability and particle reactivity. Further research is needed in order to determine not only the chemical nature of phagotrophically-regenerated species, but also the factors 
which govern the form in which trace metals are recycled by grazers. These factors may include prey nutritional status as well as protozoan metabolism.

The form in which trace metals are consumed by phagotrophs probably also has an effect on the chemical speciation of excreted metals. One striking example of this in the work presented here is in the different behavior of iron when consumed by the microflagellate Cafeteria in the form of bacterial prey vs as colloidal iron oxide. In Chapter $2,{ }^{59} \mathrm{Fe}$ regenerated from bacteria cells by the grazer Cafeteria remained very stable in the dissolved phase over extended time periods. In Chapters 3 and $5,{ }^{59} \mathrm{Fe}$ solubilized by Cafeteria from colloidal iron oxides was very particle reactive, scavenged rapidly by particle surfaces unless EDTA was added to the medium. The reasons for the different behavior of iron in these circumstances is unclear. One possibility is that the presence of colloidal iron oxides in the experiments in Chapters 3 and 5 enhanced the scavenging of iron. Another possibility is that non-specifically complexed iron is inherently more reactive than iron bound by intracellular ligands. If this is so, then the speciation of iron in the solid phase may be important in determining its bioavailability to phagotrophic organisms. For phagotrophs, scavenged or inorganic forms of iron may be an important source of bioavailable iron.

This idea brings up the other side of the coin with regard to phagotrophy as a vector for trace metal-biota interaction - the role of phagotrophy in determining the availability of trace metals to phagotrophic organisms. This thesis did not specifically address the issue of phagotrophy as a mechanism for trace metal acquisition or toxicity, focusing instead on the effects of phagotrophy on trace metal cycling. The research in this thesis has demonstrated that the phagotrophic food vacuole is a unique and ubiquitous microenvironment within the larger marine milieu, within which non-equilibrium chemical reactions of trace metals can occur. These results have important implications not only for the geochemical cycling of trace elements, but also for the role of trace elements as nutrients and toxins in the biological community. 
Phagotrophy and the paradigm of trace metal bioavailability - Our current concept of trace metal bioavailability is dominated by the free ion activity model - the availability of trace metals to biota is dependent not on total metal concentration, but rather on the concentration of free metal ions (Sunda 1988-1989). This model pre-supposes that phytoplankton (for which the model was developed) only interact with dissolved trace metals. Yet among eukaryotes (which include phytoplankton), the ability to bind and internalize macromolecules (endocytosis) and particles (phagocytosis) from the extracellular environment is an exceedingly widespread characteristic. Genetic studies have proven that the basic respiratory and photosynthetic organelles of eukaryotes (mitochondria and chloroplasts) are derived from bacterial symbionts (proteobacteria and cyanobacteria) originally phagocytized by primitive eukaryotic cells. Thus, endocytosis and phagocytosis are not only widespread but also ancient characteristics of eukaryotic cells, phenomena whose absence is a derived rather than an original condition (Raven, 1997; Ishida and Kimura, 1986).

In the planktonic environment, single-celled eukaryotes capable of endocytosis/phagocytosis are ubiquitous and important components of the microbial assemblage. Phagotrophic flagellate and ciliate protists less than $20 \mu \mathrm{m}$ in size are the major consumers of bacteria, cyanobacteria, and small algal cells (Azam et. al., 1983). Phagotrophic protists also make up an important component of the microplankton, organisms $20-200 \mu \mathrm{m}$ in size. Many planktonic protozoans are capable of employing phototrophic modes of nutrition in addition to phagotrophy. It is only recently that the abundance and ecological significance of algal phagotrophy has been recognised. This phenomenon has now been identified in five classes of phytoplankton and in ciliates (Sanders 1991; Rothhaupt 1996). Several theories have been advanced to explain phagotrophy by phototrophs, including the supplementation of carbon in low light environments, and the uptake of organic growth factors or mineral nutrients under limiting conditions. Recent findings seem to support the latter explanation, in that phosphorus limitation has been shown to stimulate phagotrophy in marine phytoflagellates (Nygaard and Tobiesen 1993), and the relative proportion of mixotrophs 
in the phytoplankton community has been found to increase in oligotrophic systems (Riemann and Christoffersen 1993; Jones 1994).

It is interesting to note that on a biomass basis, the additional "cost" for a phototroph to maintain a functioning phagotrophic apparatus is only 5-10\% (Raven, 1997). If phagotrophy is so metabolically "cheap", it may be even more widespread among phototrophs than we currently realize. A number of phototrophic thecate dinoflagellates, for example, forms not previously known to practice phagocytosis (eg. Alexandrium and Gonyaulax) have very recently been shown to ingest large particles (Jacobsen and Anderson, 1996). The presence of a complete thecal covering in these organisms, combined with the apparently patchy and episodic nature of their phagotrophic habits, had previously concealed the role of phagotrophy in their metabolism.

As outlined above, phagotrophy is likely to be more widespread among planktonic organisms than previously realized, particularly among photosynthetic forms. The research presented in this thesis has demonstrated that phagotrophic digestion as employed by heterotrophic protists is capable of solubilizing trace metals associated with a wide variety of particulate phases, both mineral and organic. As argued in Chapter 1, particles and colloids are an important reservoir for bioactive trace metals in the marine environment. Taken together, these facts imply that phagotrophy should be incorporated into our conceptual model of trace metal bioavailability, not only for heterotrophs, but for all eukaryotic cells. Trace metal-biota interaction as mediated by phagotrophy could have significant effects on the primary production and species composition of planktonic communities.

\section{Suggestions for further research}

Incorporating phagotrophy into a new paradigm of trace metal-phytoplankton interaction requires more knowledge in a number of areas, some of which are entirely physiological. At a basic level, it would be useful to know how widespread active or intermittent phagotrophy is among planktonic organisms. Clearly, such knowledge would be pertinent not only to the question of phagotrophy as a vector for trace metal-biota 
interaction, but also to the more general issues of mixotrophy as a nutritional strategy and its biogeochemical significance in nutrient cycles. Molecular biological techniques might offer a way to address this issue, by developing a probe specific for phagotrophy. Given that the basic genes encoding for phagotrophy are probably universally distributed among eukaryotes, whether they actively phagocytize or not, it would probably be necessary to target either genes induced only by active phagotrophs, or perhaps proteins involved in active phagotrophy.

A related issue of importance more specifically to the topic of phagotrophy and trace metals is to assess the turnover (i.e. ingestion rate) of particulate trace metals by phagotrophic organisms. As discussed in Chapter 5, turnover is a key parameter in protozoan-mediated effects on trace metal solids, particularly when considering inorganic species like iron oxides. It is known that heterotrophic protists will ingest a variety of non-cellular particles, including latex beads, paint particles, etc., but reports on the selectivity of phagotrophs with regard to particle consumption are conflicting (Sherr et. al., 1987; Gonzalez et. al., 1993). In addition to the consumption of discrete particles, there are issues related to the consumption of colloids associated with larger particles. Colloids have been reported to be ubiquitous in the marine environment (eg. Wells and Goldberg, 1992), and important as reservoirs of particle-reactive trace metals due to their high surface area to volume ratio. It has been suggested that bacteria are likely to be important as an adsorptive surface for colloidal material (Cho and Azam, 1990). If this is in fact so, then much of this colloidal material must be consumed by phagotrophic organisms.

Determining the turnover of particulate and colloidal trace metals by phagotrophs is a challenging prospect, since it requires not only knowledge of the ingestion rate and particle selectivity of a variety of phagotrophic and mixotrophic organisms, but also information about the physicochemical speciation of the heterogeneous assemblage of particulate and colloidal trace metals in natural waters. Clearly, a comprehensive answer to the question of trace metal turnover by phagotrophs is beyond the scope of current science. At a more limited level, progress may be made by focusing inquiry on two key 
areas related to the chemistry of phagotrophic digestion: 1) mechanistics and variability of the solubilization of solid-phase trace metals within phagotrophic food vacuoles; and 2) reactivity of naturally-occuring particulate metals within the chemical context of intracellular digestion.

In order to define the chemical context of intracellular digestion with regard to trace metal solids, it would be best to start with laboratory studies of an organism whose digestive chemistry has already been well-characterized: Paramecium (eg. Fok et. al., 1982). Given its amenability to laboratory experimentation, Paramecium could serve as a model organism to study the effects of intracellular digestion on trace metals associated with various solid phases. Based on these experiments, it should be possible to design a synthetic reaction scheme that mimics the effect of phagotrophic digestion on particles. Such a reaction scheme could then be used to assess the reactivity of naturally-occurring particulate trace metals within the chemical context of intracellular digestion. This approach is analogous to the work of Larry Mayer and colleagues on the bioavailability of sedimentary contaminants (Mayer et. al., 1995; Mayer et. al. 1996). These workers have designed a "biomimetic" assay to approximate the chemical environment of extracellular digestion in deposit feeders, and have applied this assay with great success to determine the bioavailability of contaminants in a variety of sediments to deposit feeders.

The idea of developing a biomimetic assay based on intracellular digestion for the analysis of suspended solids is attractive because chemical leaches are a common means of analyzing the speciation of trace metals on particles in the water column. Leaching methods used in previous research, however, such as dilute acetic acid, have only limited relevance to the chemical environment of intracellular digestion (eg. Landing and Lewis, 1991). A biomimetic chemical leaching procedure might provide a more meaningful context for the analysis of particulate trace metals.

In addition to bulk chemical methods, microscopic techniques could provide complementary information about the physicochemical speciation of solid phase trace metals at the level of individual particles. Microscopic methods could also be used to 
investigate the interaction of solid phase trace metals with phagotrophic organisms. Potential techniques include epifluorescent microscopy with specific chemical fluorescent probes as indicators (for example) of intracellular redox microenvironments. Electron microsopy, combined with energy dispersive elemental spectroscopy, also has potential as an analytical tool. Improved preparatory techniques have recently been developed for the analysis of aquatic particles and colloids via electron microscopy, involving the use of hydrophilic embedding resins (Perret et. al., 1991). These methods could facilitate the use of electron microscopy to study trace metal-biota interactions as mediated by phagotrophy. The analytical combination of bulk chemical extraction methods and microscopy, along with continued mechanistic experiments in model systems, will be key elements of further research on the role of phagotrophy in the marine biogeochemistry of trace elements. 


\section{References}

- Azam, F., T. Fenchel, J.G. Field, L.A. Meyer-Reil and F. Thingstad. 1983. The ecological role of water-column microbes in the sea. Mar. Ecol. Prog. Ser. 10: 257-263.

- Bruland, K.W., J.R. Donat and D.A.Hutchins. 1991. Interactive influences of bioactive trace metals on biological production in oceanic waters. Limnol. Oceanogr. 36:15551577.

- Cho, B.C. and F. Azam. 1990. Major role of bacteria in biogeochemical fluxes in the ocean's interior. Nature 332: 441-443.

- Coale, K.H. and K. W. Bruland. 1987. Oceanic stratified euphotic zone as elucidated by ${ }^{234} \mathrm{Th}:{ }^{238} \mathrm{U}$ disequilibria. Limnol. Oceanogr. 32: 189-200.

- Cornell, R.M. and U. Schwertmann. 1996. The Iron Oxides: Structure, Properties, Reactions, Occurrence and Uses. VCH, New York, NY.

- Fok, A.K., Y. Lee and R.D. Allen. 1982. The correlation of digestive vacuole $\mathrm{pH}$ and size with the digestive cycle in Parmecium caudatum. J. Protozool. 29: 409-414.

- Gonzalez, J.M., E.B. Sherr and B.F. Sherr. 1993. Differential feeding by marine flagellates on growing versus starving, and on motile versus nonmotile, bacterial prey. Mar. Ecol. Prog. Ser. 102: 257-267.

- Honeyman, B.D. and P.H. Santschi. 1989. A Brownian-pumping model for oceanic trace metal scavenging: Evidence from Th isotopes. J. Mar. Res. 47: 951-992.

- Hutchins, D.A., G.R. DiTullio and K.W. Bruland. 1993. Iron and regenerated production: Evidence for biological iron recycling in two marine environments. Limnol. Oceanogr. 38: 1242-1255.

- Ishida, K. and B. Kimura. 1986. Photosynthetic phagotrophy of Chrysophyceae:

Evolutionary aspects. Microbiol. Sci. 3: 132-135.

- Jacobson, D.M. and D.M. Anderson. 1996. Widespread phagocytosis of ciliates and other protists by marine mixotrophic and heterotrophic thecate dinoflagellates. $J$. Phycol. 32: 279-285. 
- Jones, R.I. 1994. Mixotrophy in planktonic protists as a spectrum of nutritional strategies. Mar. Micr. Food Webs. 8: 87-96.

- Landing, W.M. and B.L. Lewis 1991. Analysis of marine particulate and colloidal material for transition metals. p. 263-272. In Marine Particles: Analysis and Characterization. Hurd, D.C. and D.W. Spencer, eds. Geophysical Monograph 63.

- Mayer, L.M., L.L. Schick, T. Sawyer, C. Plante, P.A. Jumars and R.L. Self. 1995. Bioavailable amino acids in sediments: A biomimetic, kinetics-based approach. Limnol. Oceanogr. 40: 511-520.

- Mayer, L.M., Z. Chen, R.H. Findlay, J. Fang, S. Sampson, R.F.L. Self, P.A. Jumars, C. Quetel and O.F. Donard. 1996. Bioavailability of sedimentary contaminants subject to deposit-feeder digestion. Environ. Sci. Technol. 30: 2641-2645.

- Nygaard, K. and A. Tobieson. 1993. Bacterivory in algae: A survival strategy during nutrient limitation. Limnol. Oceanogr. 38: 273-279.

- Perret, D., G.G. Leppard, M. Muller, N. Belzile, R. DeVitre and J. Buffle 1991. Electron microscopy of aquatic colloids: Non-perturbing preparation of specimens in the field. Wat. Res. 25: 1333-1343.

- Raven, J.A. 1997. Phagotrophy in phototrophs. Limnol. Oceanogr. 42: 198-205.

- Reinfelder, J.R. and N.S. Fisher. 1991. The assimilation of elements ingested by marine copepods. Science 251: 794-796.

- Riemann, B. and K. Christoffersen. 1993. Microbial trophodynamics in temperate lakes. Mar. Micr. Food Webs. 7: 69-100.

- Rothhaupt, K.O. 1996. Utilization of substitutable carbon and phosphorus sources by the mixotrophic chrysophyte Ochromonas sp. Ecology. 77: 706-715.

- Sanders, R.W. 1991. Mixotrophic protists of marine and freshwater ecosystems. $J$. Protozool. 38: 76-81.

- Sherr, B.F., E.B. Sherr and R.D. Fallon. 1987. Use of monodispersed, fluorescently labeled bacteria to estimate in situ protozoan bacterivory. Appl. Env. Micobiol. 53: 958965. 
- Sunda, W.G. 1988-1989. Trace metal interactions with marine phytoplankton. Biol. Oceanogr. 6: 411-442.

- Twiss, M.R. and P.G.C. Campbell. 1995. Regeneration of trace metals from picoplankton by nanoflagellate grazing. Limnol. Oceanogr. 40: 1418-1429.

- Waite, T.D. and F.M.M. Morel. 1984a. Photoreductive dissolution of colloidal iron oxide: Effect of citrate. J. Colloid. Interface Sci. 102:121-137.

- Waite, T.D. and F.M.M. Morel. 1984b. Photoreductive dissolution of colloidal iron oxides in natural waters. Environ. Sci. Technol. 18: 860-868.

- Wells, M.L. and E.D. Goldberg, 1992. Marine submcron particles. Mar. Chem. 40: 518.

- Wells, M.L. and L.M. Mayer. 1991. The photoconversion of colloidal iron hydroxides in seawater. Deep-Sea Res. 38: 1379-1395.

AGU, Washington, D.C. 